William R. Wiley

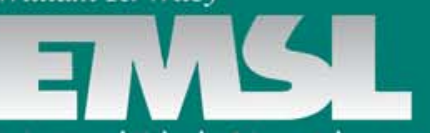

Environmental Molecular Sciences Laboratory

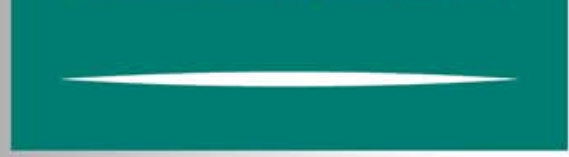

Annual Report 2000

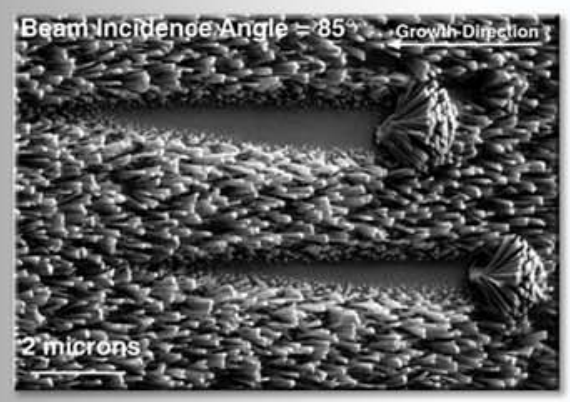

\title{
Chemical Structure and Dynamics
}

\author{
March 2001
}

Pacific Northwest

National Laboratory

Operated by Battelle for the

U.S. Department of Energy 
Cover Photo: An electron micrograph of a 1-micrometer-thick, nanoporous MgO film, demonstrating the effect of material synthesis using a molecular beam at a high angle of incidence. The beam impinges from the right at an incidence angle of $85^{\circ}$, forming a film consisting of an array of highly oriented, predominantly independent, columnar filaments tethered to the underlying substrate. The shadowing effect (the two areas where the Mgo filaments are not observed) is due to small specs of dust on the substrate that act to block the deposition.

CS\&D researchers speculate that the largely independent filaments are responsible for the film's high-temperature stability and will facilitate transport of reagents within the film. Additionally, low energy electron diffraction and $x$-ray diffraction analyses reveal that the films are crystalline. These properties make the nanoporous MgO films good candidates for further studies in heterogeneous catalysis. Precise control of the composition and structure of nanoporous materials can lead to a more fundamental understanding of reactions that occur in restricted geometries, as well as to the design and synthesis of better materials for chemical applications.

\section{DISCLAIMER}

This report was prepared as an account of work sponsored by an agency of the United States Government. Neither the United States Government nor any agency thereof, nor Battelle Memorial Institute, nor any of their employees, makes any warranty, express or implied, or assumes any legal liability or responsibility for the accuracy, completeness, or usefulness of any information, apparatus, product, or process disclosed, or represents that its use would not infringe privately owned rights. Reference herein to any specific commercial product, process, or service by trade name, trademark, manufacturer, or otherwise does not necessarily constitute or imply its endorsement, recommendation, or favoring by the United States Government or any agency thereof, or Battelle Memorial Institute. The views and opinions of authors expressed herein do not necessarily state or reflect those of the United States Government or any agency thereof.

\section{PACIFIC NORTHWEST NATIONAL LABORATORY \\ operated by \\ BATTELLE \\ for the \\ UNITED STATES DEPARTMENT OF ENERGY \\ under Contract DE-ACO6-76RLO183O}

Printed in the United States of America

Available to DOE and DOE contractors from the

Office of Scientific and Technical Information,

P.O. Box 62, Oak Ridge, TN 37831-0062;

ph: (865) 576-8401

fax: $(865) 576-5728$

email: reports@adonis.osti.gov

\footnotetext{
Available to the public from the National Technical Information Service, U.S. Department of Commerce, 5285 Port Royal Rd., Springfield, VA 22161 ph: (800) 553-6847 fax: (703) 605-6900

email: orders@ntis.fedworld.gov

online ordering: http://www.ntis.gov/ordering.htm
} 
PNNL-12154-3

UC-400

\section{Annual Report 2000}

\section{Chemical Structure and Dynamics}

Steven D. Colson, Associate Director Robin S. McD owell, Program Manager and the Staff of the Chemical Structure and Dynamics Program

April 2001

Prepared for the U.S. D epartment of Energy under Contract DE-AC06-76RL01830 


\section{Introduction}

Chemical Structure and Dynamics

Program. $1-3$

\section{Reaction Mechanisms at Liquid Interfaces}

Structure and Reactivity of Ice Surfaces and Interfaces

G. A. Kimmel, Z. Dohnálek, K. P. Stevenson, R. S. Smith, and B. D. Kay

Mixing, Transport, and Phase Separation in Deeply Supercooled, Metastable Binary Solutions P. Ayotte, Z. Dohnálek, G. A. Kimmel, R. S. Smith, and B. D. Kay.

Adsorption on Oxide Surfaces

Z. Dohnálek, G. A. Kimmel,

S. A. Joyce, P. Ayotte, R. S. Smith, and B. D. Kay

Synthesis and Characterization of

Nanoporous Thin Films Using Molecular Beam Techniques

Z. Dohnálek, G. A. Kimmel, R. S. Smith, and B. D. Kay

Scanning Electron Microscopy Studies of the Hydration of Alkali Halides

S. A. Joyce, J. T. Atherley, and J. P. Cowin

Solvation at the Oil-Water Interface, and Nano-Structured Fluids

J. P. Cowin, M. J. Iedema, and

R. C. Bell .

Soft-Landed Ion Study of Water

J. P. Cowin, M. J. Iedema, K. Wu, and A. A. Tsekouras

\section{High-Energy Processes at Environmental Interfaces}

Laser Desorption of Ground State Neutrals from Single Crystal $\mathrm{KBr}$

K. M. Beck, A. G. Joly, and W. P. Hess

Strontium Migration and Co-Precipitation at Columbia Basin Basalt/Caliche Interfacial Regions

K. M. Beck, W. P. Hess, S. M. Heald,

S. H. Withers, R. J. Peale, and

R. J. Reeder

Micro-XRF/XAS Study of Uranyl

Incorporation on Calcite 1014 Growth

Surfaces

R. J. Reeder, M. Nugent, K. M. Beck, and $S$. Heald

$\mathrm{Nd}^{3+}$ in Calcite: Evidence for Broad

Continuous Site Distribution

S. H. Withers, P. Sharek,

R. E. Peale, K. M. Beck, and

W. P. Hess

Evidence for a Common Ionization

Process in MALDI

J. Kim and W. P. Hess.

Radiolytic and Thermal Processes Relevant

to Dry Storage of Spent Nuclear Fuels

N. G. Petrik, J. P. Cowin,

T. M. Orlando, and

S. C. Marschman.

Photoionization of Liquid Water and Salt

Solutions Using a Liquid-Beam Source R. G. Tonkyn, T. M. Orlando, N. Petrik, and S. E. Barlow. 
Thermal and Radiation Stability of Hydrated Epsomite, Mirabilite, and Natron Under Europa Environmental Conditions

G. Teeter, T. M. Orlando, M. T. Sieger, N. G. Petrik, G. B. Hansen, L. Van Keulen, and T. B. McCord 3-20

\section{Cluster Models of the Condensed Phase}

Clusters and Multiply Charged Anions:

Model Studies and Solvations

L. S. Wang, X. B. Wang,

J. B. Nicholas, X. Li, X. Yang, and M. Gutowski

Probing the Electronic Structure of Atomic

Clusters and Multiply Charged Anions

L. S. Wang, X. Li, X. B. Wang,

X. Yang,H. F. Zhang, S. Liu,

A. I. Boldyrev, J. Simons

G. L. Gutsev, B. K. Rao, P. Jena,

M. Manninen, U. Landman, and

S. R. Kass.

Investigation of Metal-Carbon

Mixed Clusters

L. S. Wang, X. Li, H. F. Zhang,

H. J. Zhai, A. I. Boldyrev, and

J. Simons

\section{Single-Molecule Spectroscopy and Biological Imaging}

Development of the CARS Microscope and Application to Biological Studies

G. R. Holtom and B. D. Thrall

Combined Optical and Magnetic

Resonance Microscopy Development and

Implementation for Cellular Research

R. A. Wind, E. J. Ackerman,

G. R. Holtom, P. D. Majors, and

K. R. Minard.

Single-Molecule Dynamics of Protein-DNA Interactions in DNA Damage Recognition

H. P. Lu, L. M. Iakoucheva, and

E. J. Ackerman $5-4$
Patch-Clamp Coupled Two-Photon Fluorescence Microscopy Study of Single-

Ion Channel Dynamics and Mechanisms G. Orr, B. Thrall, S. Colson, M. Montal, and H. P. Lu

Surface-Enhanced Raman Spectroscopy on Nanoparticles and Nanostructures: Towards Vibrational Mode-Specific, Single-Molecule Dynamics

L. Zhu and H. P. Lu

Single-T4 Lysozyme Protein

Conformational and Enzymatic Reaction

Dynamics at a Cell Wall

Y. Chen and H. P. Lu.

Spatially Resolved Single Cell Irradiator to

Study Bystander Responses to Low LET

Radiation

M. Sowa Resat, J. H. Miller

J. C. McDonald, M. K. Murphy,

D. J. Strom, B. D. Thrall,

N. F. Metting, S. D. Colson, and

G. A. Kimmel

\section{Ultrasensitive and Environmental Analysis}

A Reaction Chamber Study of Alkene Chemistry: Temperature-Dependent Yield in the Ozonolysis of Ethene and Propene

R. S. Disselkamp and M. Dupuis..

High-Resolution Infrared Spectroscopy:

Basic and Applied Research

S. W. Sharpe, T. A. Blake, R. L Sams, and T. J. Johnson

Determination of Analytic Potentials from

Finite Element Computations

S. E. Barlow, A. E. Taylor, and

K. Swanson

Chemistry of Atmospheric Aerosols J. P. Cowin, M. J. Iedema, A. Laskin, M. A. Carpenter, R. S. Disselkamp,

C. M. Berkowitz, J. S. Young, and

D. Gaspar $6-10$ 


\section{Appendix}

Chemical Structure and Dynamics Staff....... 7-1

Associate Director, EMSL ............................ 7-1

Program Manager.......................................... 7-1

Staff ..................................................... 7-1

Research Scientists....................................... 7-3

Visiting Scientists .......................................... 7-4

Operations Administrator.............................. 7-4

Office Support......................................... 7-4

Postdoctoral Fellows ..................................... 7-4
Graduate Students ....................................... 7-5

Undergraduate Students ................................ 7-6

Publications and Presentations from

October 1999 ...................................... 7-6

Publications............................................. 7-6

Patents .................................................. 7-10

Presentations ….......................................... 7-10

Honors and Recognition .............................. 7-15

Collaborations........................................... 7-16

Acronyms and Abbreviations ........................ 7-20

Where CS\&D Fits in PNNL ......................... 7-22 


\section{Introduction}


This report describes the research and accomplishments of the Chemical Structure and Dynamics (CS\&D) Program from October 2000 through December 2001. Publications, Presentations, and Collaborations are listed from October 2000 to September 2002. Research is conducted in the William R. Wiley Environmental Molecular Sciences Laboratory (EMSL). EMSL is a national scientific user facility, (Figure 1.1). The Pacific Northwest National Laboratory (PNNL) in Richland, Washington operates it for the U.S. Department of Energy's (DOE's) Office of Biological and Environmental Research.

The research opportunities at EMSL are focused on the physical, chemical, and biological processes that are the foundation for addressing such critical scientific issues as environmental cleanup, energy efficiency, global climate change, and human health impacts from exposure to contaminants. More than 100 instrument systems are available for use by the research community in developing a molecular to meso-scale understanding of these processes. The uniqueness of the EMSL campus is that it offers, at one location, a comprehensive and close-coupled array of state-of-the-art capabilities and facilities enabling users to custom tailor combinations that match their specific research needs.

The EMSL complex evolved from a 1986 vision by the late Dr. William R. Wiley, director of PNNL, to build a world-class user facility enabling close synergism between the physical, mathematical, and life sciences.

Dr. Wiley and others believed that molecular level research was essential for attacking the most challenging scientific problems facing our nation in the new century. From this belief grew a concept for a center that would bring together theoreticians, modelers, and experimentalists of molecular to meso-scale level processes.

EMSL's staff provide expert assistance to users such as academic scientists, and students, as well as colleagues from other national laboratories, industry, and other government agencies. The combined experimental and intellectual capabilities provided by the EMSL environment are very important to the success of the CS\&D research program.

\section{Chemical Structure and Dynamics Program}

The Chemical Structure and Dynamics (CS\&D) program supports the Department of Energy's (DOE) mission of fostering fundamental research in the natural sciences to provide the basis for new and improved energy technologies and for understanding and mitigating the environmental impacts of energy use and contaminant releases. This research provides a foundation for understanding

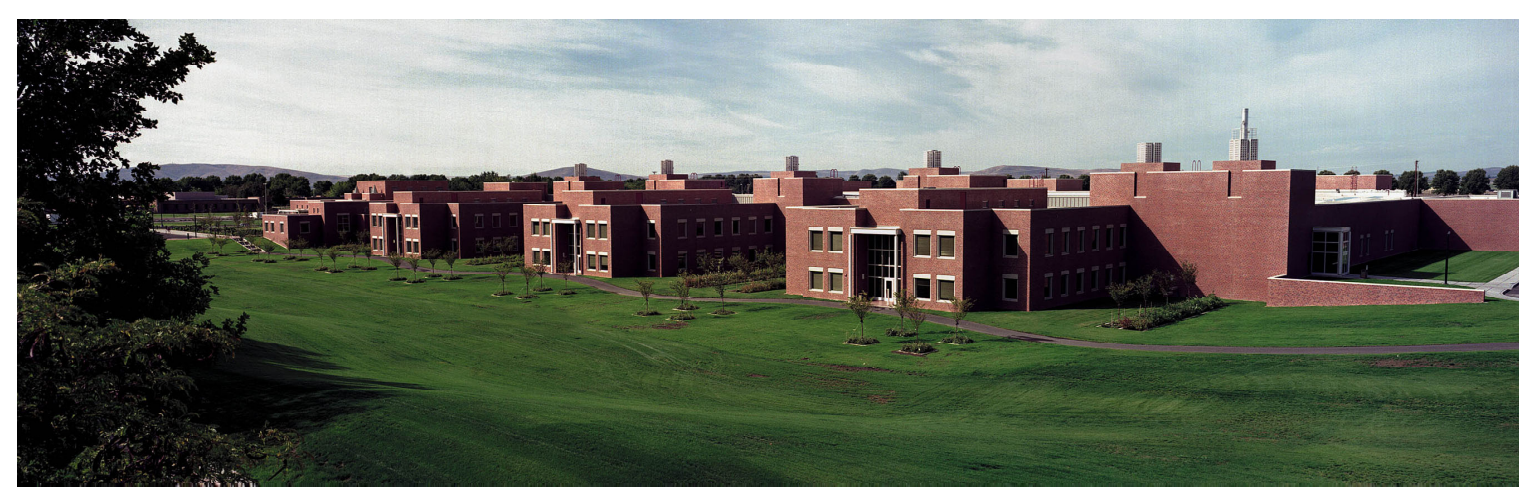

Figure 1.1. As a national scientific user facility, the EMSL supports both open and proprietary research. More information and specific procedures for becoming a user are available on the EMSL website at http://www.emsl.pnl.gov/ 
interactions of atoms, molecules, and ions with materials and with photons and electrons. Particular emphasis is on interfacial processes.

A distinguishing feature of research at the National Laboratories is their approach to problem solving. Significant scientific issues are addressed by using focused and multidisciplinary investigative teams with each team member bringing a particular skill and capability to bear on the problem. This accelerates progress. The same approach is inherent in how we manage the CS\&D Program, which involves numerous groups of scientists within the program as well as collaborators from throughout PNNL and the external scientific community.

The CS\&D Program has particular expertise in the preparation and spectroscopic analysis of molecular clusters; high-resolution biological imaging and studies of cellular processes including DNA damage and repair and lowdose radiation processes; ultra-fast and non-linear optical spectroscopies; ultra-high resolution spectroscopy for measurements of electronic and geometric structures and dynamics; surface and interface structure, chemical reaction dynamics, and kinetics; ionmolecule traps and storage technology; and specialized chambers, instruments, and models for chemical reactivity and analysis of atmospheric species including aerosols.

Our research underpins the fundamental understanding of chemical transport and reactivity in the condensed phase. It addresses the underlying uncertainties in thermal and nonthermal (i.e., radiation) chemistry, interfacial molecular and ionic transport, and other processes in complex natural and human-made systems related to energy use, environmental remediation, waste management, and understanding biological response to environmental stresses. One focus is on structures and processes of molecular and nano-scale systems in complex environments such as condensed phases and interfaces. Research guides the development of new materials and approaches for clean and efficient energy use. Another central feature is the development of innovative experimental methods with broad applications to research in the natural sciences. In addition to this core research, the CS\&D Group has expanded its interests into two major new areas: 1) the detection and monitoring of trace atmospheric species, including gaseous and particulate matter, and 2) biological processes important in the environment and health, including live cell imaging to observe the reaction dynamics of functioning biological systems in real-time, singlemolecule spectroscopy to understand proteinprotein interactions and DNA damage and repair, and novel instrumentation for studies cellular responses to low-dose radiation.

Individual papers and abstracts within each section of this report represent five research areas, listed below. The Appendix summarizes information about the CS\&D staff, visiting scientists, students, and their accomplishments.

The CS\&D Program emphasizes the following five research theme areas:

- Reaction Mechanisms at Solid, Liquid, and Gas Interfaces. This research area focuses on developing fundamental molecular information about processes occurring at the interfaces between environmentally important liquids, solids, and gases.

- High-Energy Processes at Environmental Interfaces. This research area focuses on obtaining a mechanistic understanding of chemical transformations of molecules and materials driven by thermal, radioactive, or optical sources.

- Cluster Models of the Condensed Phase. This research is aimed at providing a molecular-level understanding of solvation and subsequent reactions in simple and complex systems as they relate to the chemistry of complex wastes, contaminated solids and groundwater, and other systems found in nature. 
- Single-Molecule Spectroscopy and Biomolecular Sciences. This research emphasizes single-molecule spectroscopy and high-resolution biological imaging techniques for studying biological systems.
- Ultrasenstive and Environmental Analysis. This research area focuses on developing a suite of instrumentation for fast, accurate, highly sensitive, and discriminatory real-time analyses of chemical and biological natural or human-made species. 


\section{Reaction Mechanisms at Solid, Liquid, and Gas Interfaces}

This research area focuses on developing fundamental molecular information about processes occurring at the interfaces between environmentally important liquids, solids, and gases. For example, a common element in environmental restoration is the need to understand molecular processes 1) in aqueous solutions and at the interface between aqueous and organic solutions and 2) at the interface between aqueous solutions and environmentally important solid materials.

Molecular processes occurring at liquid-liquid interfaces also play an important role in the subsurface transport of contaminants such as organic solvents (e.g., chlorinated hydrocarbons) released into soil and groundwater. Processes at the gas-liquid interface are critical in atmospheric transport processes.

Model systems such as amorphous solid water permit detailed studies of solvation and the effects of solvation on chemical reactivity. This provides information about intermolecular interactions that lay the foundation for accurate modeling of solution processes. Studies provide information about the factors controlling the rates of reactions in solution essential to predicting the fate of contaminants in aqueous environments and essential to influencing the selectivity of ligands for specific ions important in developing separations agents for waste treatment.

It's also crucial to understand molecular processes at the interface between aqueous solutions and environmentally important materials, such as aerosol particles, minerals, and glasses. This addresses fundamental science questions about contaminant fate and transport, and waste immobilization. Because most environmental materials are in an oxidized form, we emphasize the structure and chemistry of oxide materials, especially naturally occurring oxides of silicon, aluminum, and iron. The adsorption of species on and their incorporation in soil minerals affect their transport. Mineral interfaces can enhance or retard reactivity as well as transform contaminants. Knowledge of molecular processes at solid-liquid interfaces is also important to understand the stability of glasses proposed for encapsulating high-level radioactive waste. Over the long half-lives of the radionuclides, water can degrade these waste glasses resulting in higher than expected releases of radionuclides. 


\section{Structure and Reactivity of Ices, Oxides, and Amorphous Materials at Surfaces and Interfaces}

\author{
J. Daschbach, P. Ayotte, ${ }^{(a)}$ \\ G. Kimmel, B. Peden, ${ }^{(b)}$ \\ G. Schenter, ${ }^{(c)}$ R. S. Smith, and \\ B. D. Kay
}

Supported by DOE Office of Basic Energy

Sciences, Chemical Sciences Division.

(a) NSERC Postdoctoral Fellow.

(b) ERULF summer student.

(c) Theory, Modeling, and Simulation.

The objective of this program is to examine physiochemical phenomena occurring at the surface and within the bulk of ices, oxides, and amorphous materials. The microscopic details of physisorption, chemisorption, and reactivity of these materials are important to unravel the kinetics and dynamic mechanisms involved in heterogeneous (i.e., gas/liquid) processes. This fundamental research is relevant to heterogeneous atmospheric chemistry, astrophysical dust and comet chemistry, solvation and liquid solutions, and deeply supercooled liquids. Our research provides a quantitative understanding of the kinetic processes in these complex systems. These results can be used directly as the basis for developing mathematical kinetic models of these complicated systems. For example, the reactivity and solvation of polar molecules on ice surfaces play an important role in complicated reaction processes that occur in the atmosphere. These same molecular processes are key to understanding dissolution, precipitation, and crystallization kinetics in multiphase, multicomponent, complex systems.

Amorphous materials are important in a variety of scientific disciplines such as chemistry, physics, materials science, and biology. Amorphous solid water (ASW) is of special importance for many reasons, including the open question over its applicability as a model for liquid water and fundamental interest in the properties of glassy materials. In addition to the properties of ASW itself, understanding the intermolecular interactions between ASW and an adsorbate is important in such diverse areas as solvation in aqueous solutions, cryobiology, and desorption phenomena in cometary and interstellar ices.

Our approach is to use molecular beams to synthesize "chemically tailored" nanoscale films as model systems to study ices, amorphous materials, supercooled liquids, and metal oxides. In addition to their utility as a synthetic tool, molecular beams are ideally suited for investigating the heterogeneous chemical properties of these novel films. Modulated molecular beam techniques will enable us to determine the adsorption, diffusion, sequestration, reaction, and desorption kinetics in realtime. These elementary molecular-level kinetic processes are germane to a broad range of technologically and environmentally relevant phenomena. In support of the experimental studies, kinetic modeling and Monte Carlo simulation techniques are used to analyze and interpret the experimental data.

\section{Molecular Beam Study of Helium Diffusion in Ice}

The interactions of gas phase molecules with the ice surface are an important aspect of the overall chemical reactivity that occurs on ice particles.

Many factors influence these interactions including pressure, temperature and structure of the ice surface. The Molecular Beam Scattering Instruments allow us to control many of these variables in order to determine the fundamental physics involved.

In previous work we observed that it is possible to embed $\mathrm{He}$ atoms in both crystalline ice and ASW using $\mathrm{He}$ beams with kinetic energies in the range $0.065-0.215 \mathrm{eV}$. Experimental measurements and theoretical calculations show that helium will only "enter" at a specific region of the ice surface and that the absorption probability increases with the collision energy. This implies that there is an energetic barrier to helium absorption. The He atoms are embedded in the solid water substrate held at $22 \mathrm{~K}$ where they are stable over experimentally accessible time frames. The experimental measurement and trajectory simulations of the absorption probability data are in qualitative agreement at high collision energies, but at low 
collision energies the experiments show a much higher absorption probability. This may be due to the onset of an alternative adsorption mechanism at low temperature.

To investigate what happens to the embedded $\mathrm{He}$, we studied the diffusion of $\mathrm{He}$ in ASW. The transport of the small closed shell atom $\mathrm{He}$ in water and ice provides important data for the theoretical description of the structure and dynamics of these systems. By using molecular beam deposition of nanoscale thin films of $\mathrm{H}_{2} \mathrm{O}$ we have been able to determine the diffusion coefficient of $\mathrm{He}$ in ASW over the temperature range 70-115 K.

Thin ASW layers (10 monolayers [ML]) were grown with the $\mathrm{H}_{2} \mathrm{O}$ beam at normal incidence to a $\operatorname{Pt}(111)$ substrate at $22 \mathrm{~K}$. Subsequently, the thin ASW layer is loaded with He by impinging a monoenergetic $\mathrm{He}$ beam at normal incidence. Following this, another $\mathrm{H}_{2} \mathrm{O}$ deposition is made at $22 \mathrm{~K}$, capping the underlying $\mathrm{He}$ embedded in the $\mathrm{H}_{2} \mathrm{O}$ film. Cap thicknesses are varied over the range 50-10,000 ML. Diffusion of helium through the film is examined by heating the sample with a linear temperature ramp and detecting the desorption of He using a line-of-sight quadrapole mass spectrometer.

The cap overlayer impedes the diffusion of $\mathrm{He}$ atoms to the ASW/vacuum interface. The low aspect ratio of the thin ASW films $\left(3.0 \times 10^{-6}\right.$ to $6.0 \times 10^{-4}$ ) allows the use of a one-dimensional model. The transport is modeled as a single layer of embedded $\mathrm{He}$ atoms located between the first two monolayers of the underlying ASW film with the $\operatorname{Pt}(111)$ substrate treated as a perfectly reflecting boundary for the $\mathrm{He}$ atoms, and an activated form is assumed for the He transport $\mathrm{D}=\mathrm{D}_{\mathrm{o}} \exp \left(-\mathrm{E}_{\mathrm{a}} / \mathrm{RT}\right)$.

Figure 2.1 shows a series of experiments with cap thicknesses in the range 100-10,000 ML. The solid lines are the result of a simulation using a

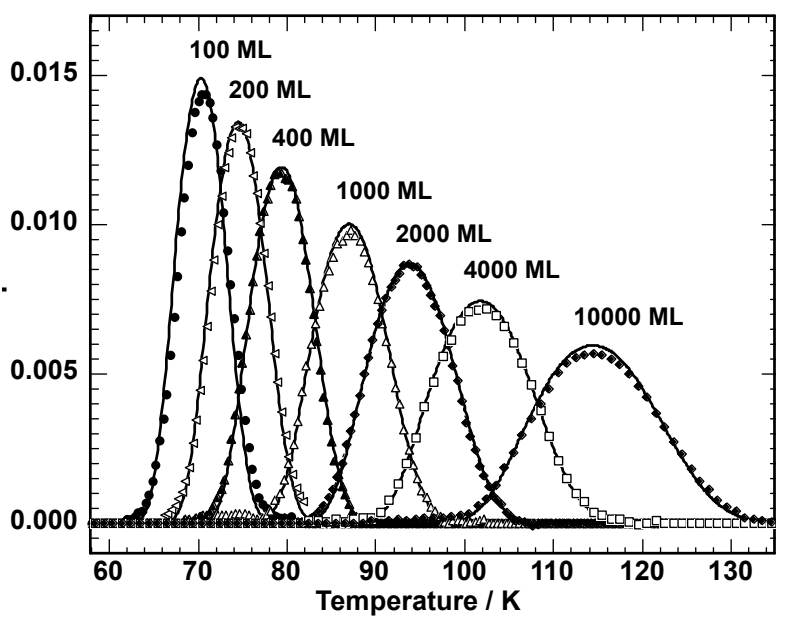

Figure 2.1. Linear temperature He desorption traces for He embedded in a $10 \mathrm{ML}$ thick ASW slab at $22 \mathrm{~K}$ with beam energy of $0.108 \mathrm{eV}$ with ASW cap layers of 100, 200, 400, 1000, 2000, 4000 , and $10,000 \mathrm{ML}$ at a ramp rate of $2.0 \mathrm{~K} / \mathrm{s}$. The data are shown with points and the results of simulations performed for the respective experimental conditions and with $D_{0}=1.85 \times 10^{-3} \mathrm{~cm}^{2} / \mathrm{s}$ and $E_{a}=0.127 \mathrm{eV}$ are shown with lines.

single optimized set of Arrhenius parameters, $D_{0}$ and $\mathrm{E}_{\mathrm{a}}\left(1.85 \times 10^{-3}\right.$ and $0.127 \mathrm{eV}$ respectively $)$. The simulated curves fit the data well and a sensitivity analysis indicates the activation energy is accurate to better than 5 percent.

Figure 2.2 shows an Arrhenius plot of this data along with values determined for liquid water (Jähne et al. 1987) and ice (Haas et al. 1971). All of the data show similar activation energies with the apparent $D_{0}$ values following a trend which is reasonable for the expected behavior of the $\mathrm{H}_{2} \mathrm{O}$ bulk, with the ASW results falling between the two orthogonal directions in ice $\mathrm{I}_{\mathrm{h}}$ and the liquid $\mathrm{H}_{2} \mathrm{O}$ data somewhat higher. Uncertainties in the prior data, mostly due to the more limited inverse temperature ranges investigated, make more extensive analysis based on the $D_{0}$ values premature. 


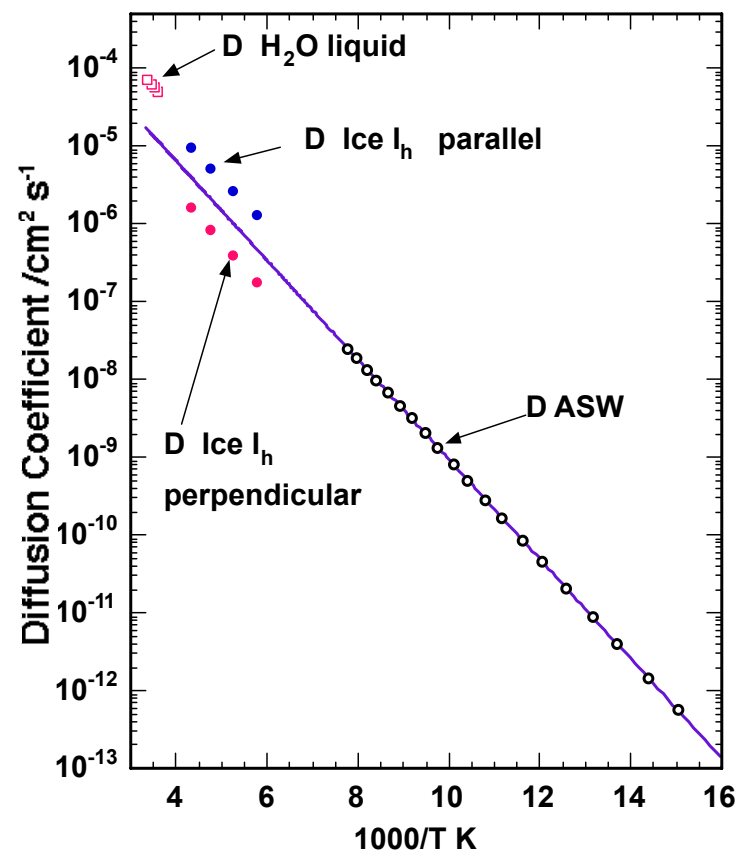

Figure 2.2. An Arrhenius plot showing the data from this work and results from the literature for the diffusion of $\mathrm{He}$ in liquid water (Jähne et al. 1987) and ice $I_{h}$ (Haas et al. 1971).

\section{Molecular Beam Study of Absorption, Desorption, and Clustering of Water on $\mathrm{Pt}(111)$ Surface}

Helium specular scattering is a sensitive probe of adsorbate coverage and morphology on metal substrates in the sub-monolayer regime. This is due to the large He-adsorbate scattering cross section, $\sigma$, that can be substantially larger than the square of the substrate or the dense packed adsorbate lattice parameter. Hence, the specular helium reflectivity will be a function of both the number density and morphology of the adsorbate. Two limiting cases are especially important in understanding the structure of adsorbates: pure random adsorption gives rise to an exponential attenuation of the helium reflectivity with adsorbate coverage, while complete two-dimensional clustering yields a linear attenuation with adsorbate coverage.

We have studied the initial clustering of water on $\mathrm{Pt}(111)$ as part of our ongoing efforts to understand the kinetics and thermodynamics of phase changes in supercooled liquids and as an important step in our efforts to investigate the kinetics of wetting/dewetting. These measurements are able to clearly show the morphological change in the $\mathrm{H}_{2} \mathrm{O}$ adsorbate layer as a function of the substrate temperature.

The measurements were performed in a molecular beam scattering chamber with a base pressure $\leq 1.0 \times 10^{-10}$ torr. Two quadrupally pumped molecular beams were employed: a $300 \mathrm{~K}$ supersonic He beam $45^{\circ}$ with respect to the sample normal and a $300 \mathrm{~K}$ effusive $\mathrm{H}_{2} \mathrm{O}$ beam at $30^{\circ}$. Detection of the specular He signal was with a quadrapole mass spectrometer with $\pm \pm 5^{\circ}$ angle of acceptance centered on the He specular direction. The Pt(111) sample was resistively heated and temperature control was stable to better than $\pm 0.01 \mathrm{~K}$ throughout any experiment. The sample was flashed to $1000 \mathrm{~K}$ prior to each experiment and cooled to the operating temperature in under $230 \mathrm{~s}$. Experiments were performed after $20 \mathrm{~s}$ of temperature stabilization by recording the specular He intensity as a function of time. After a short period of He scattering from the clean surface, the water beam was turned on and the attenuation of the He signal due to water adsorption was monitored. The $\mathrm{H}_{2} \mathrm{O}$ flux was calibrated using temperature programmed desorption (TPD) and observing the fluence at the onset of the $\mathrm{H}_{2} \mathrm{O}$ multilayer desorption feature. The sticking coefficient of $\mathrm{H}_{2} \mathrm{O}$ on clean $\mathrm{Pt}(111)$ is close to unity and converges rapidly to unity with $\mathrm{H}_{2} \mathrm{O}$ coverage; therefore the data reported here assumes unity sticking throughout.

Figure 2.3 shows He scattering as a function of $\mathrm{H}_{2} \mathrm{O}$ coverage for a series of substrate temperatures. At $29.95 \mathrm{~K}$ over the coverage range 0 to $0.80 \mathrm{ML}$, the data is fit exactly by a single exponential yielding a cross section of $31.6 \AA^{2}$. At $125.0 \mathrm{~K}$ the data is linear with $\mathrm{H}_{2} \mathrm{O}$ coverage up to $\sim 0.9 \mathrm{ML}$. The traces at $70.0 \mathrm{~K}$ and $95.0 \mathrm{~K}$ fall between these two cases. The $29.95 \mathrm{~K}$ and $125.0 \mathrm{~K}$ data match the analytical models for scattering from a randomly located adsorbate and a fully two-dimensionally clustered adsorbate respectively. 


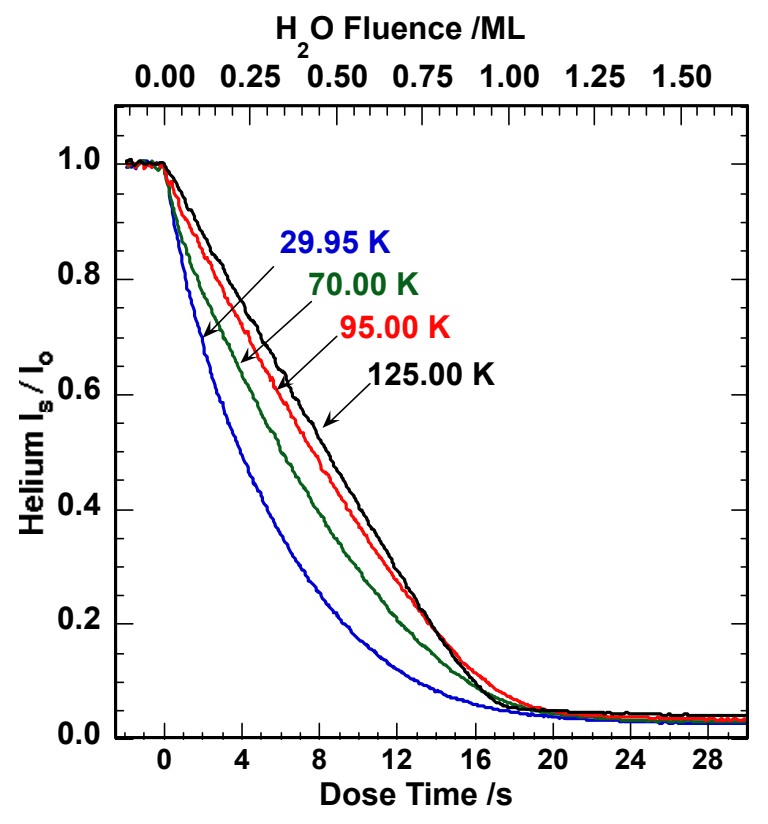

Figure 2.3. Specular He scattering measured from $\mathrm{Pt}(111)$ during $\mathrm{H}_{2} \mathrm{O}$ deposition as a function of time (lower abscissa), $\mathrm{H}_{2} \mathrm{O}$ fluence (upper abscissa) and temperature. The effusive $\mathrm{H}_{2} \mathrm{O}$ beam impinged on the sample at $30^{\circ}$ with a flux of $0.056 \mathrm{ML} / \mathrm{s}$.

Figure 2.4 shows the initial He scattering cross section calculated from the first 5 percent of $\mathrm{H}_{2} \mathrm{O}$ coverage. At temperatures below about $70 \mathrm{~K}$ the measured initial cross section is close to the $31 \AA^{2}$ value determined at $29.95 \mathrm{~K}$ which corresponds to randomly deposited $\mathrm{H}_{2} \mathrm{O}$. At temperatures above about $80 \mathrm{~K}$ the measured initial cross section is close to the $10 \AA^{2}$ measured at $125.0 \mathrm{~K}$ corresponding to $\mathrm{H}_{2} \mathrm{O}$ which is fully clustered in two dimensions.

These experiments should help us understand the molecular-level interaction of water with a variety hydrophobic and hydrophilic materials. For example, the extent to which water will wet the hydrophobic portion of a macromolecule depends on the strength of the water/ molecule interaction. The strength of the water/molecule energetics will also determine the structural and physical

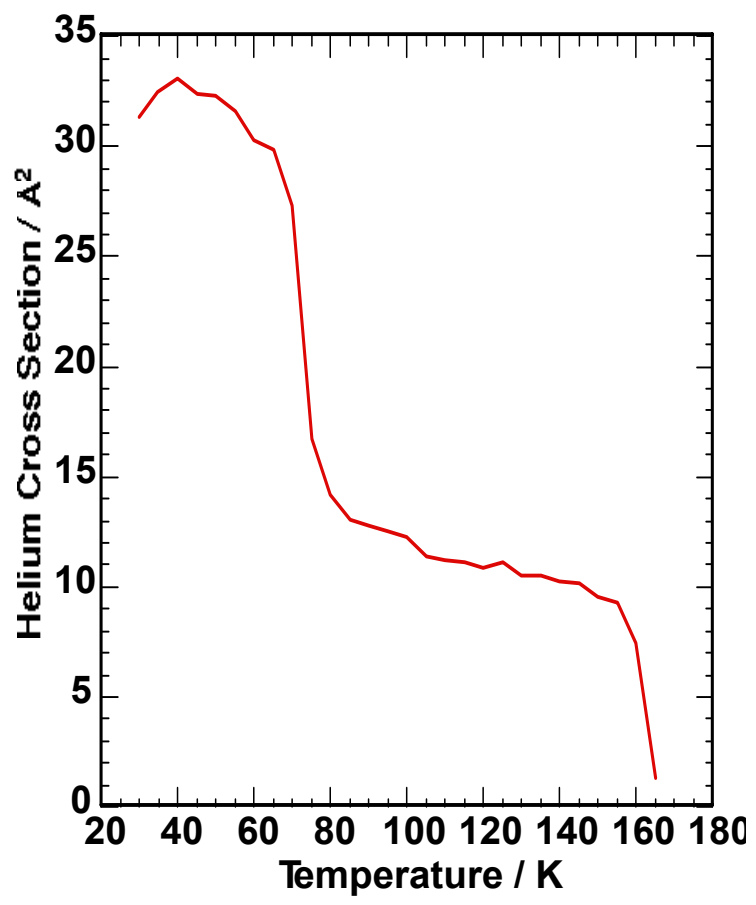

Figure 2.4. Initial He scattering cross section for $\mathrm{H}_{2} \mathrm{O}$ on $\mathrm{Pt}(111)$ as a function of temperature.

properties of the first water layer. When the water-water interaction is stronger than the watersubstrate interaction, the water molecules prefer to bind to themselves and the system is said to be hydrophobic or nonwetting. The extent to and rate with which molecules coalesce will depend on the height of the energetic barriers to diffusion. By varying the incident water beam flux and substrate temperature, we hope to study the nucleation and clustering of adsorbed water molecules on both hydrophobic and hydrophilic substrates.

\section{References}

Jähne, B., G. Heinz, and W. Dietrich, J. of Geophysical Research 92, 10767 (1987).

Haas, J., B. Bullemer, and A. Kahane, Solid State Communications 9, 2033 (1971). 


\section{Mixing, Transport, and Phase Separation in Deeply Supercooled, Metastable Binary Solutions}

\author{
P. Ayotte, ${ }^{(a)}$ R. S. Smith, \\ G. Teeter, ${ }^{(b)} Z$. Dohnálek, \\ G. A. Kimmel, and B. D. Kay
}

Supported by DOE Office of Basic Energy

Sciences, Chemical Sciences Division.

(a) NSERC Postdoctoral Fellow.

(b) BES Funded Postdoctoral Fellow.

While solvation plays a fundamentally important role in chemical reactions in the environment and in the laboratory, the microscopic details of solvation and dissolution are poorly understood. The success of our previous research on thin films of amorphous solid water demonstrates that nanoscale films of beam deposited amorphous materials are useful for understanding phenomena occurring in deeply supercooled liquids (Smith and Kay 1999; Smith et al., 2000). The use of deeply supercooled liquids allows for the determination of the details of the solvation dynamics and for the separation of enthalpic and entropic contributions. The next step was to extend this technique to study the kinetics of mixing and solvation that occurs in deeply supercooled binary solutions.

\section{Deeply Supercooled Solutions of Methanol and Ethanol}

Supercooled liquids are metastable and their lifetimes are dictated by the kinetics for crystallization. Traditional experimental studies have used a variety of methods to suppress crystallization while cooling from the liquid phase. An alternative approach is to heat an amorphous solid above its glass transition temperature, $T_{g}$, whereupon it transforms into a deeply supercooled liquid prior to crystallization.

We chose the methanol/ethanol binary system to demonstrate the utility of our approach. At room temperature, this system behaves ideally and as such makes an excellent test case for studying its supercooled solution. We find that compositionally tailored nanoscale films of glassy methanol and ethanol exhibit complete diffusive intermixing and suppressed crystallization when heated above $T_{g}$. Furthermore, the resulting containerless liquids evaporate as continuously mixed ideal binary solutions while retaining their solid-like macroscopic shapes.

Nanoscale amorphous binary films of methanol (M) and ethanol (E) were grown at $20 \mathrm{~K}$ on a $\mathrm{Pt}$ (111) single crystal in an ultra-high vacuum chamber using molecular beam techniques. The molecular beam allows for precise calibration of the deposited amount. At this temperature and at normal incidence, the vapor-deposited material forms a dense amorphous solid (Stevenson et al. 1999). Composite films having a diameter of $\sim 6 \mathrm{~mm}$ were grown by sequential dosing of methanol and ethanol. Experiments where methanol was deposited on top of ethanol (M/E) and where ethanol was deposited on top of methanol (E/M) were conducted. The composite films were then heated and the desorption of methanol and ethanol was measured mass spectrometrically. Concurrent evaluation of the condensed phase chemical composition and physical state (amorphous vs. crystalline) was performed using reflection-absorption infrared spectroscopy (RAIRS).

Comparison of the desorption traces from composite films grown with alternate dosing sequences (i.e., $\mathrm{M} / \mathrm{E}$ vs. $\mathrm{E} / \mathrm{M}$ ) yield information about the extent of diffusive intermixing. In the absence of intermixing, desorption from these composite films would result in the species deposited on top evaporating first and impeding the evaporation from the species deposited underneath. Complete intermixing would result in desorption traces independent of the initial dose order. Incomplete intermixing results in complex desorption traces as described previously (Smith et al. 1997). The results from thermal desorption experiments on composite methanol-ethanol films were independent of dose order for films as thick as 300 total layers $(\sim 100 \mathrm{~nm})$. Furthermore, initially layered binary amorphous films displayed identical results to those from samples that were grown with molecular beams made from premixed vapors. These observations indicate that initially layered amorphous films have completely intermixed prior to the onset for measurable methanol desorption $(\mathrm{T} \sim 120 \mathrm{~K})$. 
Given the observed intermixing, it is possible to estimate a lower limit for the diffusivity of the binary films using the relation $\mathrm{D} \sim \mathrm{L}^{2} / \mathrm{t}$. Mixing occurs over a distance of $100 \mathrm{~nm}$ in approximately $10 \mathrm{sec}$, requiring diffusivities in excess of $10^{-11} \mathrm{~cm}^{2} / \mathrm{s}$ at $\mathrm{T} \sim 120 \mathrm{~K}$. This diffusivity is consistent with extrapolations of the pure liquid diffusivities to this temperature and about a millionfold smaller than their diffusivities at room temperature. This finding indicates that the individual amorphous solid constituents of the sample intermix and exhibit liquid-like behavior on the nanoscale while the sample maintains its macroscopic solid-like shape.

Figure 2.5 shows time sequences of the $\mathrm{OH}$ stretching region during isothermal annealing experiments for various films. The FTIR spectra for both the pure ethanol (top set) and pure methanol (bottom set) show analogous behavior. The first spectrum in both series (tail of arrow) displays the broad band characteristic of an amorphous solid, while subsequent spectra show the

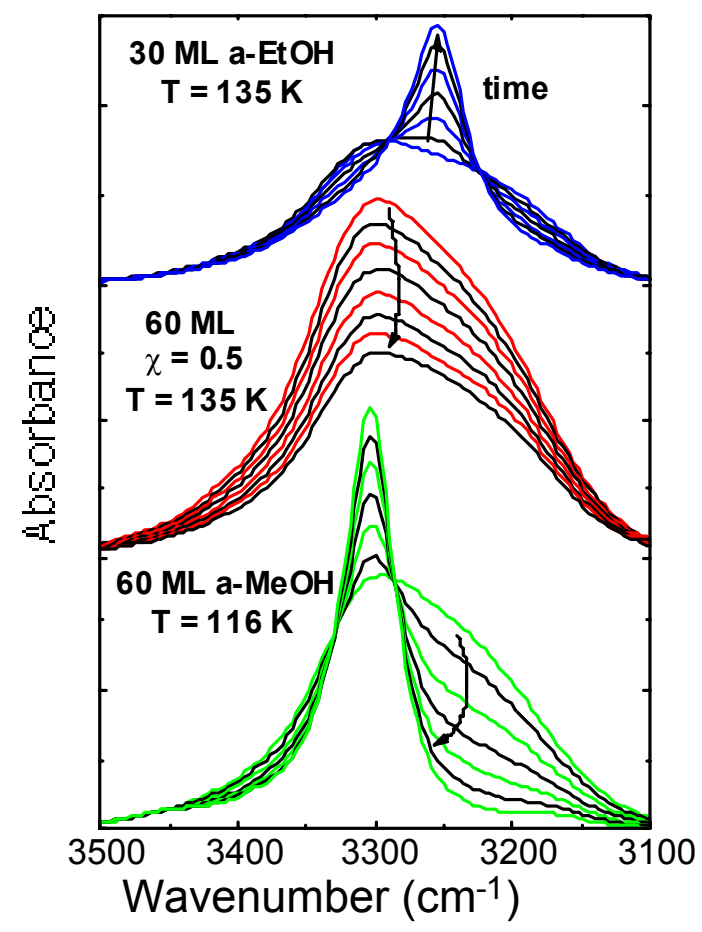

Figure 2.5. A series of infrared spectra for initially amorphous thin films of ethanol (top), ethanol and methanol (middle), and methanol (bottom). The spectra show that the pure films crystallize whereas the mixed film does not. emergence of narrower peaks. The last spectrum in each time sequence corresponds to the fully crystallized film. Both sets of spectra display isosbestic points, indicating the conversion from the metastable amorphous to the stable crystalline phase.

In contrast to the pure films, the spectra for an initially layered binary amorphous film (middle set) are observed to remain amorphous-like for the duration of the experiment at $135 \mathrm{~K}$. The lack of crystallization in this composite film indicates that intermixing preceded and thereby inhibited crystallization that occurs readily in pure amorphous methanol or ethanol. This behavior is consistent with the persistent supercooling observed in methanol-ethanol liquid mixtures.

The inhibition of crystallization in methanolethanol mixtures makes them well suited for studying deeply supercooled liquid solutions formed from nanoscale amorphous solids. The evaporation kinetics from these composite films provide a stringent test for whether or not these systems behave as homogeneous liquid solutions. The TPD spectra for a series of methanol/ethanol composite films with varying initial methanol mole fractions are shown in Figure 2.6. The evaporation of the film is described quantitatively by a kinetic model describing evaporation from a continuously mixed ideal binary liquid solution. The agreement between the experiment (symbols) and the ideal solution kinetic model (lines) is excellent. These experiments occur at temperatures below the melting point of the binary mixture indicating ideal behavior for the supercooled liquid solution.

These results demonstrate that methanol and ethanol exhibit complete diffusive intermixing and suppressed crystallization when heated above $T_{g}$. Furthermore, the resulting containerless liquid evaporates as a continuously mixed ideal binary solution while retaining its solid-like macroscopic shape.

We have shown that amorphous thin films can be useful in determining the properties of liquids and solutions in temperature regimes previously unattainable. The proof-of-principle experiments to 


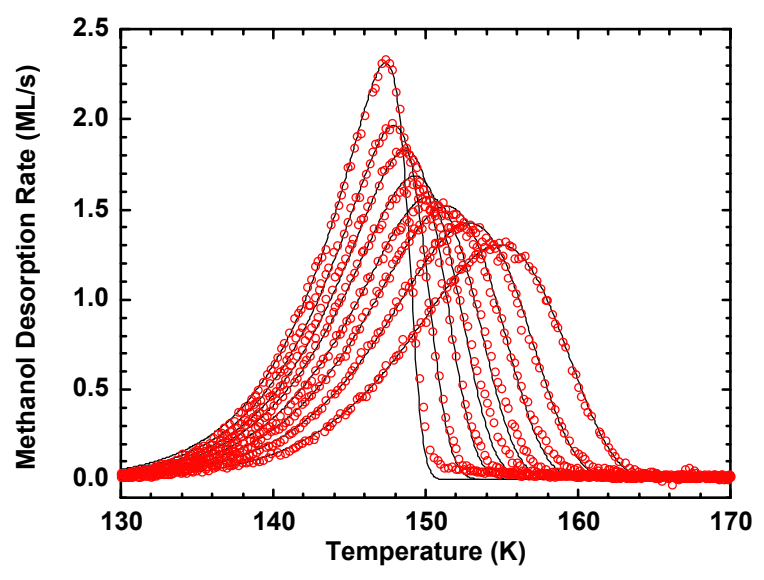

Figure 2.6. Methanol TPD spectra from binary thin films of methanol (30 ML) and ethanol (various) with initial methanol mole fractions between 0.15 and 0.85 . The symbols are the experimental data and the lines are the ideal solution model simulation.

date demonstrate that nanocscale films of beamdeposited amorphous materials are promising for understanding phenomena occurring in liquid aqueous solutions. In an effort to understand the molecular-level interactions that occur in liquid solutions, we will begin a systematic study binary mixtures of alcohol/water ices grown using molecular beam deposition. This work will evolve to include the more complex binary mixtures such as $\mathrm{ROH} / \mathrm{H}_{2} \mathrm{O}, \mathrm{NH}_{3} / \mathrm{H}_{2} \mathrm{O}$ and $\mathrm{HCl} / \mathrm{H}_{2} \mathrm{O}$. We will also implement the optical components necessary to provide in-situ FTIR vibrational spectroscopic capabilities. The combination of molecular beam dosing, mass spectrometric detection, and in-situ FTIR spectroscopy should enable us to unravel the mechanistic details of mixing and solvation kinetics in aqueous solutions.

\section{References}

Smith, R. S. and B. D. Kay, Nature 398, 788-791 (1999).

Smith, R. S., Z. Dohnalek, G. A. Kimmel, K. P. Stevenson, and B. D. Kay, Chemical Physics 258, 291-305 (2000).

Stevenson, K. P., G. A. Kimmel, Z. Dohnalek, R. S. Smith, and B. D. Kay, Science 283, $1505-$ 1507 (1999).

Smith, R. S., C. Huang, E. K. L. Wong, and B. D. Kay, Phys. Rev. Lett, 79, 909, (1997).

\section{Adsorption Dynamics and Desorption Kinetics on Oxide Surfaces}

\author{
Z. Dohnálek, R. S. Smith, and \\ B. D. Kay
}

Supported by DOE Office of Basic Energy

Sciences, Chemical Sciences and Materials

Sciences Divisions.

The objective of this program is to examine chemical phenomena occurring at model oxide surfaces. Oxide interfaces are important in the subsurface environment; specifically, molecularlevel interactions at mineral surfaces are responsible for the transport and reactivity of subsurface contaminants at Hanford. Unfortunately, our molecular-level understanding of oxide surface chemistry is severely lacking. Adsorption at surfaces of metal oxides has recently been the focus of considerable experimental attention, in part due to the wide- ranging importance of these materials in environmental and industrial processes. Metal oxides are often used as catalysts or as supports for catalysts, making the interaction of adsorbates with their surfaces of much interest. In addition, metal oxides are increasingly being used in other industrial applications, such as materials for gas sensors and electronic devices.

Here we use techniques such as molecular beam epitaxy and chemical vapor deposition to grow epitaxial thin oxide films on single-crystal metallic substrates. The successful synthesis of such crystalline thin oxide films will enable the entire arsenal of electron-based surface analytical techniques to be applied to the characterization of these non-conducting materials. These studies employ variable-energy supersonic molecular beams to determine energy-, angle-, and coveragedependent trapping and/or dissociation probabilities.

\section{Adsorption Dynamics and Desorption Kinetics of Argon and Methane on $\mathrm{MgO}(100)$}

The dynamics and kinetics of adsorption and desorption of molecules from surfaces play an important role in many heterogeneous processes 
having both fundamental and technological importance. Catalysis, gaseous corrosion, and microelectronics fabrication, for example, are processes that involve reactive gas-surface scattering with enormous economic and strategic importance. Nonreactive gas-surface interactions form the atomistic basis for such macroscopically relevant phenomena as aerodynamic drag and heat transfer. Over the past two decades considerable experimental and theoretical progress has been made in understanding the dynamics of both molecular and dissociative adsorption on metal surfaces.

While the structure and thermodynamic properties of adsorbates on metal oxide surfaces have been explored using a broad range of experimental and theoretical techniques, the dynamics and kinetics of adsorbate-metal oxide interactions are not well understood. For example, even the dynamics relevant to the physisorption of weakly bound species on well-ordered single crystal oxide surfaces is largely unexplored. The paucity of experimental dynamical studies is related to difficulties in the preparation of ordered, high quality oxide surfaces as well as problems associated with the rapid cooling of these low thermal conductivity crystals. To circumvent these problems we employ high-quality $\mathrm{MgO}(100)$ thin films, grown epitaxially on Mo(100) ( Dohnálek et al. 2001).

The desorption kinetics of $\mathrm{Ar}$ and $\mathrm{CH}_{4}$ from $\operatorname{Mg}(100)$ were studied using temperature programmed desorption (TPD). For brevity, those results are only summarized here. The TPD spectra for both $\mathrm{Ar}$ and $\mathrm{CH}_{4}$ were analyzed to extract the coverage dependent binding energy of these adsorbates to $\mathrm{MgO}(100)$. This analysis involves direct mathematical inversion of the TPD spectra using the Polanyi-Wigner equation. As we demonstrate below, the adsorption of both $\mathrm{Ar}$ and $\mathrm{CH}_{4}$ on $\mathrm{MgO}(100)$ does not involve overcoming an activation barrier hence, the desorption energies extracted from the TPD experiments are a measure of the adsorbate binding energy to the $\mathrm{MgO}(100)$ surface. Analysis of the TPD spectra yields desorption energies of $8.5 \pm 1.4$ and $13 \pm 2 \mathrm{~kJ} / \mathrm{mole}$ for $\mathrm{Ar}$ and $\mathrm{CH}_{4}$, respectively, in excellent agreement with prior experimental and theoretical values. Both $\mathrm{Ar}$ and $\mathrm{CH}_{4}$ are weakly bound to the $\mathrm{MgO}(100)$ surface. As expected for physisorption, the ratio of the $\mathrm{CH}_{4}$ to the $\mathrm{Ar}$ binding energy $(\sim 1.53)$ is nearly identical to the ratio of their polarizabilities (1.58).

The adsorption dynamics of $\mathrm{Ar}$ and $\mathrm{CH}_{4}$ on $\mathrm{MgO}(100)$ were studied using molecular beamsurface scattering techniques. Figure 2.7 displays a representative subset of analyzed data illustrating the coverage and energy dependence of $\mathrm{Ar}$ (Figure 2.7a) and $\mathrm{CH}_{4}$ (Figure 2.7b) trapping on $\mathrm{MgO}(100)$ at $22 \mathrm{~K}$. For both $\mathrm{Ar}$ and $\mathrm{CH}_{4}$, the trapping probability decreases with increasing kinetic energy, and increases with increasing coverage. We find that at the highest kinetic energies the trapping probability does not reach unity $(1.00 \pm 0.02)$ until completion of the third monolayer.

The observation that the trapping probability increases with increasing coverage indicates that

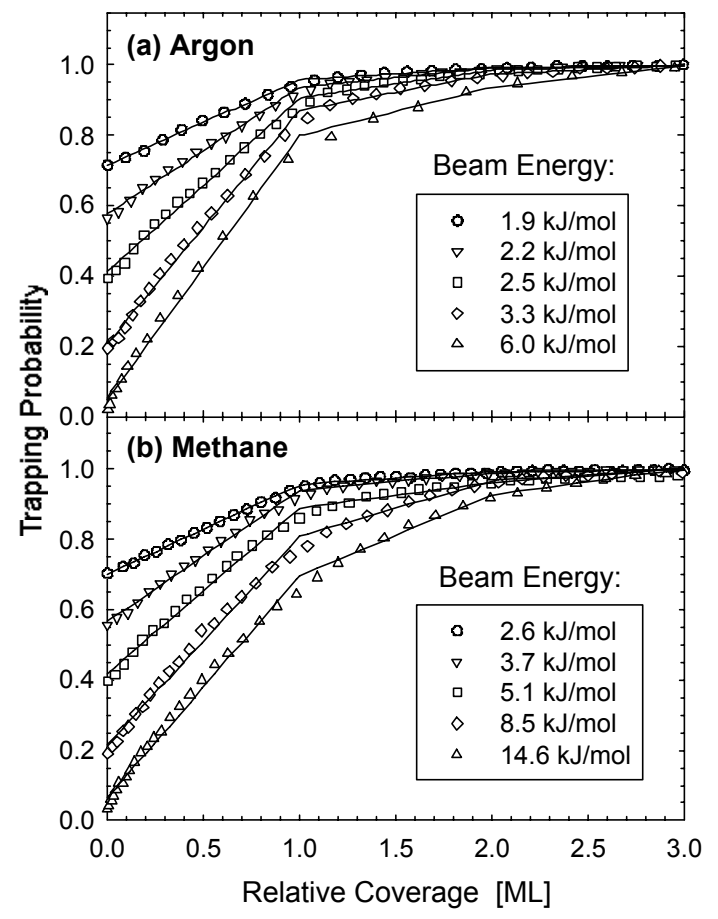

Figure 2.7. Coverage dependent trapping probabilities of $\mathrm{Ar}(\mathrm{a})$ and $\mathrm{CH} 4$ (b) on $\mathrm{MgO}(100)$ at $22 \mathrm{~K}$ for various incident beam energies, $\mathrm{EB}$, as tabulated. Solid lines represent fits to the adsorbate assisted trapping model described in the text. 
energy transfer between the incident projectile (Ar or $\left.\mathrm{CH}_{4}\right)$ and the $\mathrm{MgO}(100)$ substrate is less efficient than collisions between the incident projectile and an adsorbed particle of it own kind. Such adsorbate assisted trapping is a natural consequence of the kinematics and dynamics of collision processes. The adsorption kinetics associated with this mechanism can be described using a simple model where adsorption may occur only directly or through an extrinsinic precursor state.

If every particle that traps on a site occupied by an adsorbate, migrates to, and adsorbs on an unoccupied site in a lower layer prior to desorbing, the trapping dependence can be described by a simple model. The solid lines displayed in Figure 2.7 represent fits of this simplified layer-by-layer model to the experimental data. Clearly, this simple model does a very good job describing the experimental data.

The dependence of the trapping probability on coverage and incident angle was examined for both $\mathrm{Ar}$ and $\mathrm{CH}_{4}$. For both species, measurements were performed at five angles $(0,15,30,45$, and 60 degrees) and several different kinetic energies. Figure 2.8 displays a representative subset of the data for Ar (Figure 2.8a) and $\mathrm{CH}_{4}$ (Figure 2.8b). As clearly illustrated, the trapping probability is independent of incident angle over the entire coverage range between 0 and $3 \mathrm{ML}$. Although not shown specifically here, the coverage dependent trapping probability is independent of incident angle over the entire range of kinetic energies examined. Hence, the disposal of incident beam kinetic energy attendant with trapping on the $\mathrm{MgO}(100)$ does not depend on the angle which the impinging $\mathrm{Ar}$ and $\mathrm{CH}_{4}$ particles impact the surface. This finding is in marked contrast to the behavior observed for the initial trapping probability of $\mathrm{Ar}$ and $\mathrm{CH}_{4}$ on $\mathrm{Pt}(111)$ where the trapping probability increases for more oblique impacts.

The dependence of the initial trapping probability on incident angle is often used as a measure of the lateral corrugation of the underlying potential energy hypersurface which governs the trapping

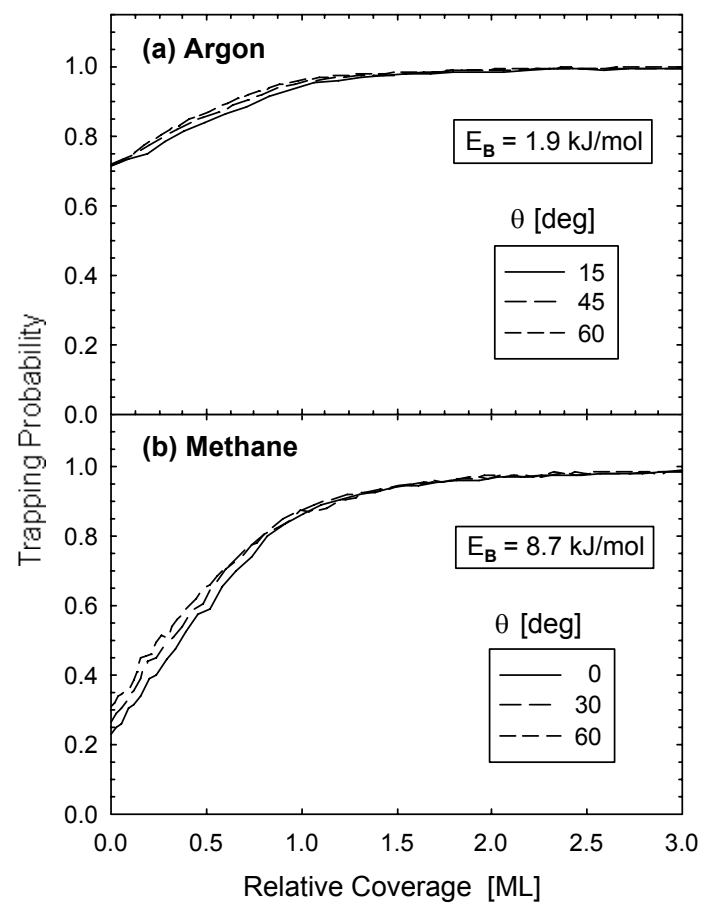

Figure 2.8. Coverage dependent trapping probabilities for $\mathrm{Ar}(\mathrm{a})$ and $\mathrm{CH}_{4}$ (b) on $\mathrm{MgO}(100)$ at $22 \mathrm{~K}$ as a function of incident beam angle.

dynamics. In "normal energy scaling" only translational energy associated with momentum directed perpendicular to the surface is efficiently exchanged during the initial trapping process, whereas in "total energy scaling" both parallel and perpendicular components of the incident projectile's momentum are equally important in determining the trapping dynamics.

In our case, the initial trapping probability for both $\mathrm{Ar}$ and $\mathrm{CH}_{4}$ decreases dramatically with increasing kinetic energy. Furthermore, for a fixed kinetic energy, the trapping probability does not depend on the incident angle of the impinging beam flux. Together these findings indicate that the initial trapping probability exhibits total energy scaling. Figure 2.9 displays the initial trapping probability for Ar plotted versus (a) total collision energy and (b) the normal collision energy. The data clearly exhibit almost perfect "total energy scaling." Analogous results are obtained for the initial trapping probability of 


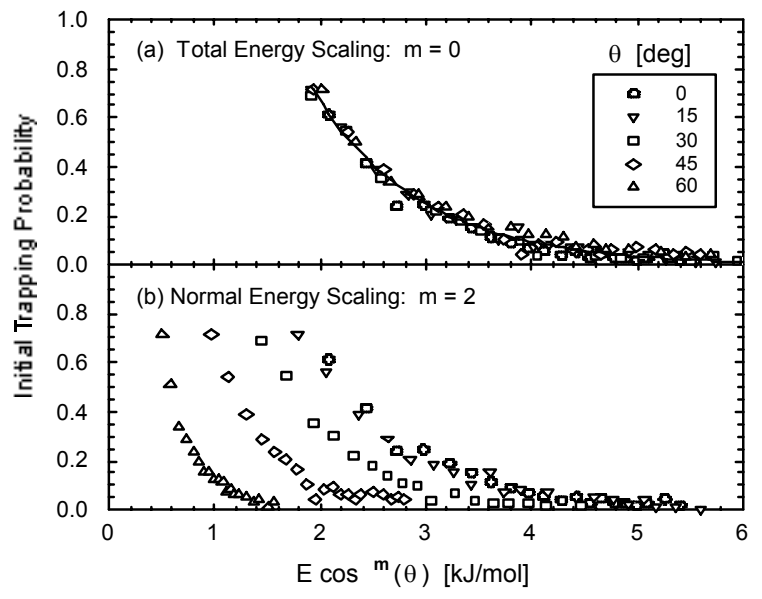

Figure 2.9. Initial trapping probability for $\mathrm{Ar}$ on $\mathrm{MgO}(100)$ at $22 \mathrm{~K}$ as a function of angle-scaled beam kinetic energy for various incident beam angles, $\theta$, as tabulated. Data scaled using "total energy scaling", $(m=0)$, and "normal energy scaling", $(m=2)$, are plotted in panels $(a)$ and (b), respectively.

$\mathrm{CH}_{4}$. This is very likely due to the presence of a potential energy surface having a large degree of lateral corrugation, consistent with recent theoretical calculations on these systems.

The observation that the initial trapping probabilities for $\mathrm{Ar}$ and $\mathrm{CH}_{4}$ on $\mathrm{MgO}(100)$ obey "total energy scaling" is in sharp contrast to recent results for $\mathrm{Ar}$ and $\mathrm{CH}_{4}$ trapping on $\mathrm{Pt}(111)$ which exhibit nearly perfect "normal energy scaling". These differences suggest that the adsorbatesubstrate interactions on $\mathrm{MgO}(100)$ are laterally corrugated, whereas the analogous interactions on $\mathrm{Pt}(111)$ are relatively smooth. Despite the presence of a corrugated interaction potential, both Ar and $\mathrm{CH}_{4}$ are sufficiently mobile on $\mathrm{MgO}(100)$ to diffuse to, and preferentially bind at, morphological defects during a typical TPD experiment. This level of lateral mobility is in agreement with theoretical calculations that indicate facile adsorbate diffusion on the corrugated $\mathrm{MgO}(100)$ surface.

\section{Reference}

Dohnálek, Z., G. A. Kimmel, S. A. Joyce, P. Ayotte, R. S. Smith, and B. D. Kay J. Phys. Chem. B. 105, 3747-3751 (2001).

\section{Synthesis and Characterization of Nanoporous Thin Films Using Molecular Beam Techniques}

\author{
Z. Dohnálek, G. A. Kimmel, \\ D. E. McCready, ${ }^{(a)}$ \\ J. S. Young, ${ }^{(a)}$ A. Dohnálková, ${ }^{(b)}$ \\ R. S. Smith, and B. D. Kay \\ Supported by PNNL Laboratory Directed \\ Research and Development (LDRD) and by \\ DOE Office of Basic Energy Sciences, \\ Chemical Sciences Division. \\ (a) Interfacial Chemistry and Engineering. \\ (b) Environmental Microbiology.
}

We have demonstrated the growth of compositionally and structurally tailored materials with extremely high porosities in amorphous solid water thin films (Kimmel et al. 2001a, 2001b; Stevenson et al. 1999). The effects of the porosity on the uptake, sequestration, release, and reactivity of gases on these highly porous materials will be studied. This work may have important applications in many areas including heterogeneous catalysis, reactive transport through porous media, capillary condensation, competitive adsorption, phase transitions, and chemical reactions in confined and/or restricted geometries.

The interaction of gases and fluids with these nanoscale films forms the basis for a variety of the useful applications of porous materials. For example, the rates of uptake, sequestration, reaction, and release of gases in these materials often determines their performance characteristics in devices such as catalysts and chemical sensors. A detailed understanding of the relationship between these kinetic processes and the morphology of the material is lacking. For these materials, the transport of reagents through the pores and the catalytic reactions are intimately coupled. Molecular beams have historically been used to study heterogeneous kinetic processes on non-porous materials. The time-resolved, modulated beam techniques we propose are ideally suited to study the coupled transport/reaction processes occurring in nanoporous materials. Understanding these 
processes is essential for the rational design and synthesis of materials for particular applications. The important parameters for the heterogeneous growth of materials include the impingement flux, the energy and angle of the impinging molecules, chemical nature of the substrate, and the substrate temperature. The instrumentation in our laboratory is designed to precisely control all of these parameters.

\section{Structural and Chemical Characterization of Aligned Crystalline Nanoporous MgO Films Grown via Reactive Ballistic Deposition}

We have recently developed a reactive ballistic deposition technique for synthesizing highly porous $\mathrm{MgO}$ films. These thin films have been characterized by $\mathrm{N}_{2}$ adsorption and have surface areas as high as $1000 \mathrm{~m}^{2} / \mathrm{g}$. The surface areas are essentially unchanged for temperatures below $1200 \mathrm{~K}$. The film consists of an array of highly oriented, predominantly independent, columnar filaments tethered to the underlying substrate. We speculate that the largely independent filaments are responsible for the observed high-temperature stability and will give rise to facile transport of reagents within the film.

Additionally, low energy electron diffraction (LEED) and $\mathrm{x}$-ray diffraction (XRD) reveal that the films are crystalline. The feather-like structure of the individual filaments indicates that porous $\mathrm{MgO}$ has a very high surface area desirable for possible chemical applications is shown in Figure 2.10. As a first step in the chemical characterization, we have studied the physisorption of $\mathrm{N}_{2}$ by the nanoporous $\mathrm{MgO}$ films. In general, physisorption is a very sensitive probe of the interaction between the substrate and the adsorbate and is thus a good probe of the the relevant chemistry will occur. Physisorption also allows us to measure the adsorption capacity distribution of binding sites on the surface where of the porous films, i.e., to measure the total number of adsorption sites. The surface area information obtained is analogous to that from $\mathrm{N}_{2}$ BrunauerEmmett-Teller (BET) isotherm measurements.

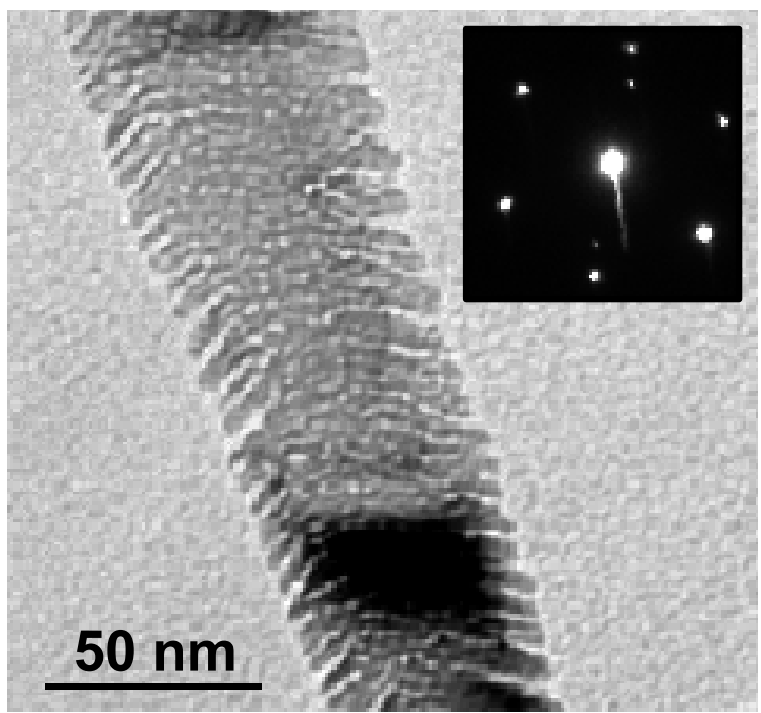

Figure 2.10. An HRTEM image of an MgO filament. The image reveals the feather-like internal pore structure typical of filaments from the film shown in above. A selected area diffraction pattern demonstrating the crystalline nature of the filament is shown in the inset.

The TPD spectra for $\mathrm{N}_{2}$ on a dense monocrystalline $\mathrm{MgO}(100)$ film, a nanoporous $\mathrm{MgO}$ deposited at $200 \mathrm{~K}$, and the same nanoporous $\mathrm{MgO}$ film after annealing to $900 \mathrm{~K}$ are shown in Figure 2.11. The $\mathrm{N}_{2}$ TPD from the dense monocrystalline $\mathrm{MgO}(100)$ surface is dominated by a single desorption feature at $42 \mathrm{~K}$ which corresponds to the desorption of $\mathrm{N}_{2}$ from $\mathrm{MgO}(100)$ terraces.

In contrast to the dense $\mathrm{MgO}(100)$ film, the $\mathrm{N}_{2}$ desorption from a nanoporous $\mathrm{MgO}$ film grown at $200 \mathrm{~K}$ occurs over a very broad temperature range $(25-140 \mathrm{~K})$, indicating a wide range of binding sites. After annealing to $900 \mathrm{~K}$, the nanoporous film retains its high surface area as shown in Figure 2.11. However, the $\mathrm{N}_{2} \mathrm{TPD}$ lineshape, which was previously broad and unresolved, displays a series of four relatively welldefined peaks, at $\sim 32,42,56$, and $98 \mathrm{~K}$, of $\mathrm{N}_{2}$ condensed within the nanopores of individual filaments. Preliminary studies on porous films show that the percentage of defect sites is greatly increased compared to smooth dense films.

A simple physical mechanism, ballistic deposition, can be used to understand the dependence of 


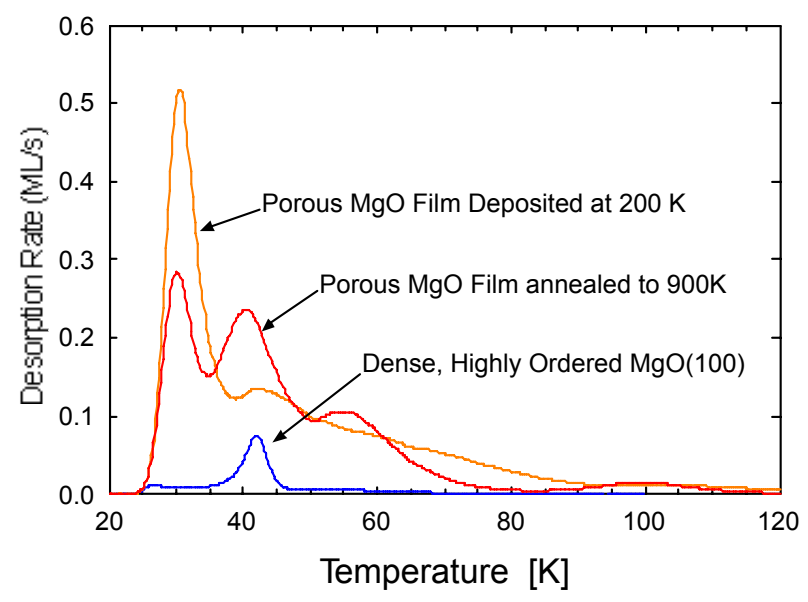

Figure 2.11. Temperature programmed desorption spectra of $\mathrm{N}_{2}$ physisorbed on thin $\mathrm{MgO}$ films. All films contain an equal amount of $\mathrm{MgO}$ equivalent to 40 layers of dense $\mathrm{MgO}(100)$. The spectra are the $\mathrm{N}_{2}$ TPD from dense monocrystalline $\mathrm{MgO}(100)$, the $\mathrm{N}_{2}$ TPD from nanoporous $\mathrm{MgO}$ grown at $200 \mathrm{~K}$ and $85^{\circ}$. and the $\mathrm{N}_{2}$ TPD obtained after annealing film grown at $200 \mathrm{~K}$ to $900 \mathrm{~K}$.

morphology on the growth angle. The basic premise of ballistic deposition is that molecules incident from the gas phase stick at the first site they encounter at the surface of the solid without subsequent diffusion. Ballistic deposition at or near normal incidence results in rough surfaces due to the stochastic nature of the deposition process. At grazing incidence, deposition can not occur in regions behind high points on the surface due to simple shadowing, resulting in the formation of columnar, porous materials. Increasing the surface temperature during growth leads to formation of dense, smooth films through enhanced surface diffusion. Therefore, by controlling the temperature, flux, and angle of deposition, we can control the morphology of the film. In order to make a useful catalytic material, the chemical composition of the nanoporous material must also be controlled. For example, multiple molecular beams could be used to simultaneously control the oxide pore structure and the location of the catalytically active metal site during synthesis.

As we have demonstrated above, the reactive ballistic deposition (RBD) technique can be used to synthesize extremely porous (90 percent), high surface area $\left(1000 \mathrm{~m}^{2} / \mathrm{g}\right)$, thermally robust (1200 $\mathrm{K})$, nanoporous, highly textured, crystalline $\mathrm{MgO}$ films. The surface area of these films is far greater than that of fine powders $\left(2-100 \mathrm{~m}^{2} / \mathrm{g}\right)$ of crystalline $\mathrm{MgO}$. Due to the physical simplicity of the RBD technique we anticipate that it should be applicable to the synthesis of a broad range of chemically-tailored, catalytically active materials. This work has been recently published (Dohnálek et al. 2002) and was featured on the cover of April 11, 2002, issue of The Journal of Physical Chemistry B (see Figure 2.12).

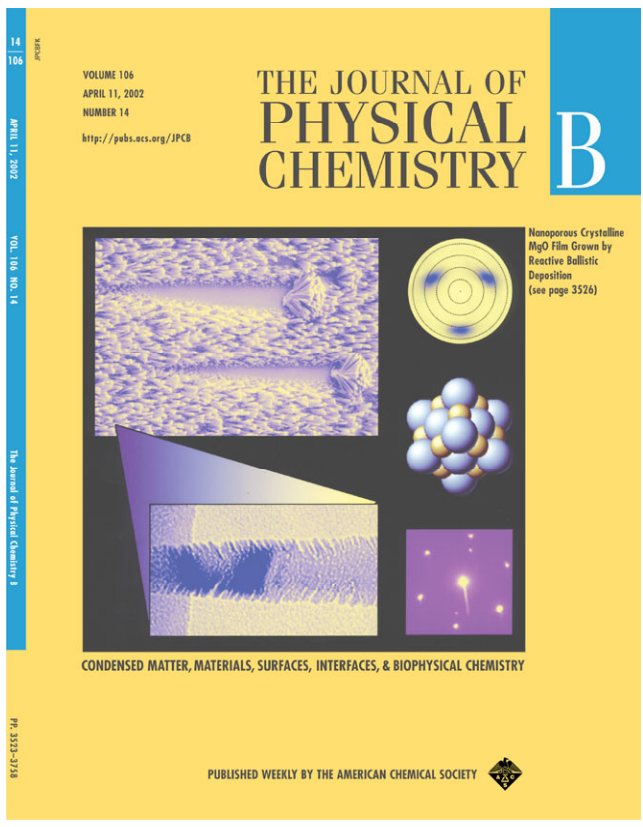

Figure 2.12. The April 11, 2002 cover of The Journal of Physical Chemistry $B$ highlights the work discussed here.

\section{References}

Dohnálek, Z., G. A. Kimmel, D. E. McCready, J. S. Young, A. Dohnálkova', R. S. Smith, and B. D. Kay, J. Phys. Chem. B 106, 3526-3529 (2002).

Kimmel, G. A., K. P. Stevenson, Z. Dohnálek, R. S. Smith, and B. D. Kay, J. Chem. Phys. 114, 5284 (2001a).

Kimmel, G. A., K. P. Stevenson, Z. Dohnálek, R. S. Smith, and B. D. Kay J. Chem. Phys. 114, 5295 (2001b).

Stevenson, K. P., G. A. Kimmel, Z. Dohnalek, R. S. Smith and B. D. Kay, Science 283, 15051507 (1999). 


\section{Solvation at the Oil Water Interface and Nano- Structured Fluids}

\author{
J. P. Cowin, M. J. Iedema, \\ R.C. Bell (postdoctoral fellow), \\ H. Wang (postdoctoral fellow)
Supported by the DOE Office of Basic Energy Sciences.

Liquids within a few nanometers of an interface or in nanopores have different properties than bulk forms of the same liquid. This makes it difficult to predict phenomena as diverse as ion transport across cell membranes, charge separation at a liquid-solar-converter interface, lubrication properties of oils, or the solvent-borne reactions that take place inside nanotextured materials like zeolites. We have used the soft landing ion source to probe the ion solvation, transport, and trapping at interfaces.

The soft landing ion source is a unique, very low energy ion beam that produces mass-selected, aqueous-type molecular ions at $1 \mathrm{eV}$. This "chemist's ion beam" can gently land ions on or in solvent films to recreate ionic interfaces with unprecedented control. It was built in collaboration with Professor Barney Ellison at the University of Colorado, Boulder.

Applications of the soft-landing ion beam typically follow the approach seen in Figure 2.13. Composite solvent films of thickness from a few to 1000 s of monolayers thick are first grown at temperatures too low for solvent diffusion to occur. Then ions are deposited, and more solvent can then be added. Upon warming, the ions can move by their attraction to the substrate due to their collective field and ion mobility.

\section{Ion Solvation at the Oil-Water Interface}

Typically hydration and solvation strongly affect both the energetics and kinetics of ion transport. This is particularly important to ion transport in living cells, multi-phase chemistry, etc. Understanding this has been complicated by constraints

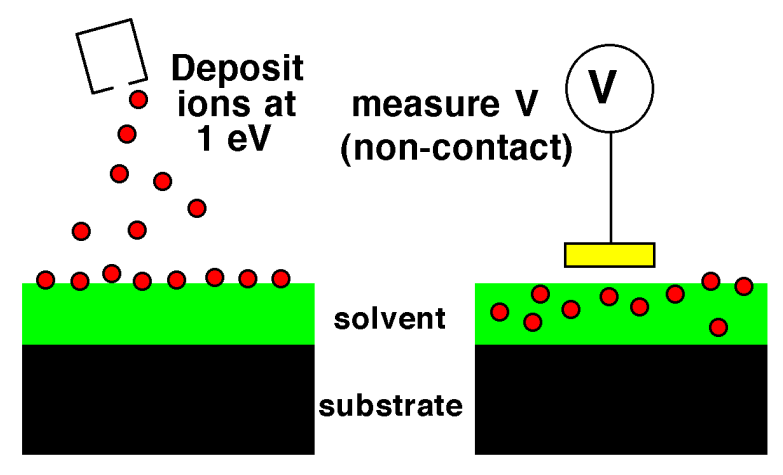

Figure 2.13. Soft landing ions. At left ions are deposited at $1 \mathrm{eV}$ onto prepared cryogenic films of glassy organic, water, or mixed/composite films. At right, thermal and field-induced ion motion is followed via work function changes $\Delta \phi$ as the film is warmed. $\Delta \phi$ scales as the average ion height in the film.

of charge neutrality, difficulties of probing the buried interface, and the low solubility of ions in most organic liquids

We used our soft landing ion source to make direct experimental observations of the effect of hydration on the motion of hydronium ions across a water-oil interface. Shown in Figure 2.14, at the far left, we fabricate nano-composite films of oil (3-methylpentane [3MP]/water/oil/metal) to probe ion motion. Hydronium ions are placed on top at $30 \mathrm{~K}$ where they generate a collective, planar electric field. As the assembly is warmed above the glass transition temperature of $3 \mathrm{MP}$, this field will pull the ions through the composite. The middle figure shows the ions after they become trapped at the water layer, and then eventually escape as it is further warmed. At right, we monitor the ion position via electrostatics(Kelvin probe), and find that the ions escape anywhere from 90 to $125 \mathrm{~K}$, depending on the electric field pulling on the ions. The $\mathrm{E}$ field varies from 0.21 to $3.9 \times 10^{8} \mathrm{~V} / \mathrm{m}$ from right to left.

The solvation of ions in the water layer creates a potential minimum at the water layer position, as shown in Figure 2.15 (curve labeled "0.00"). The ions are assumed trapped at the position of the circle with the cross. The electric field that the ions create is strong enough to deform the trap potential - the more ions present, the higher the 

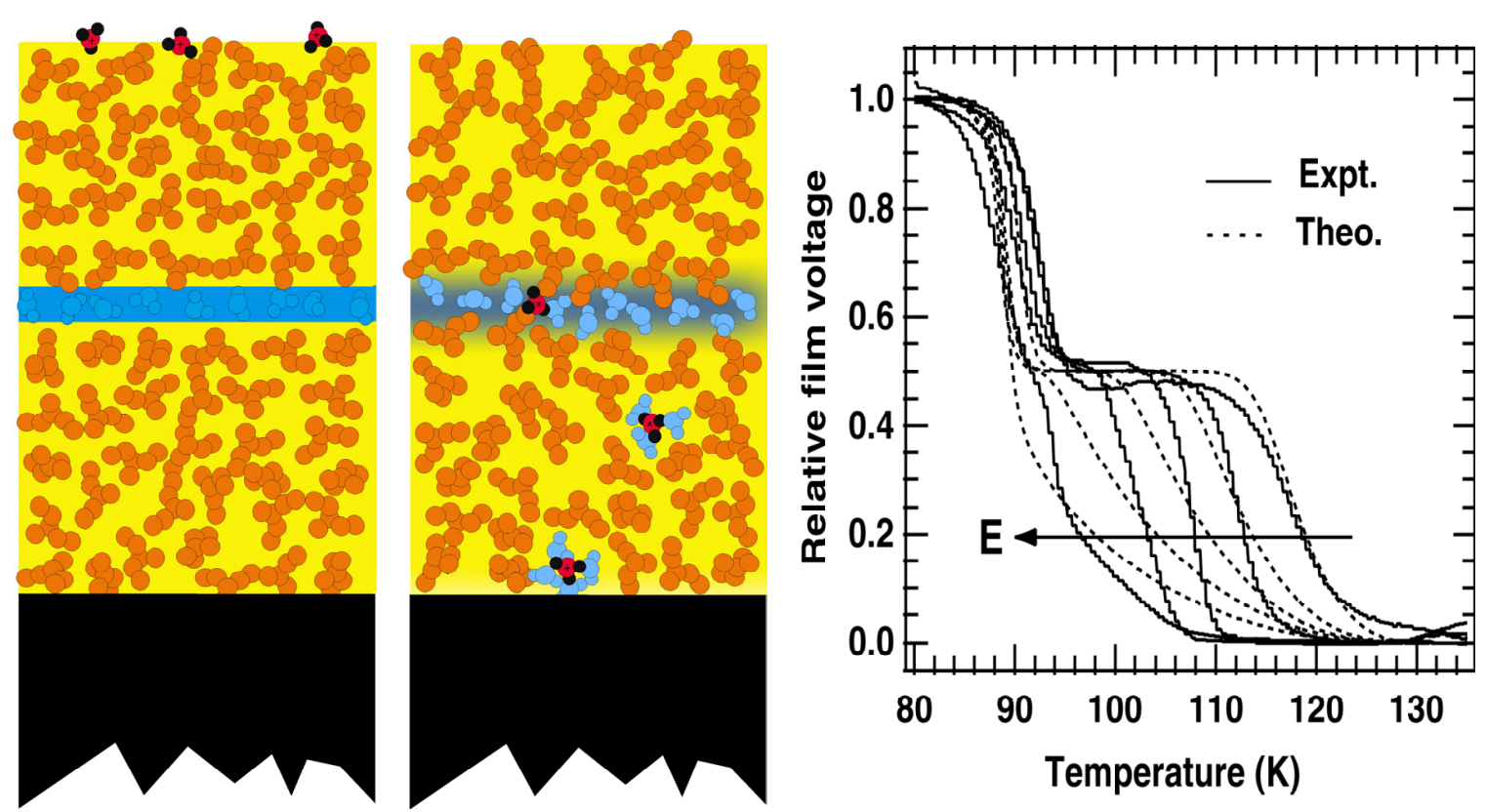

Figure 2.14. Solvation at the oil-water interface. At left, nano-fabricated films of water/oil/water probe ion solvation. At right, escape from the water "trap" occurs from 90 to $125 \mathrm{~K}$, depending on electric forces on ions.

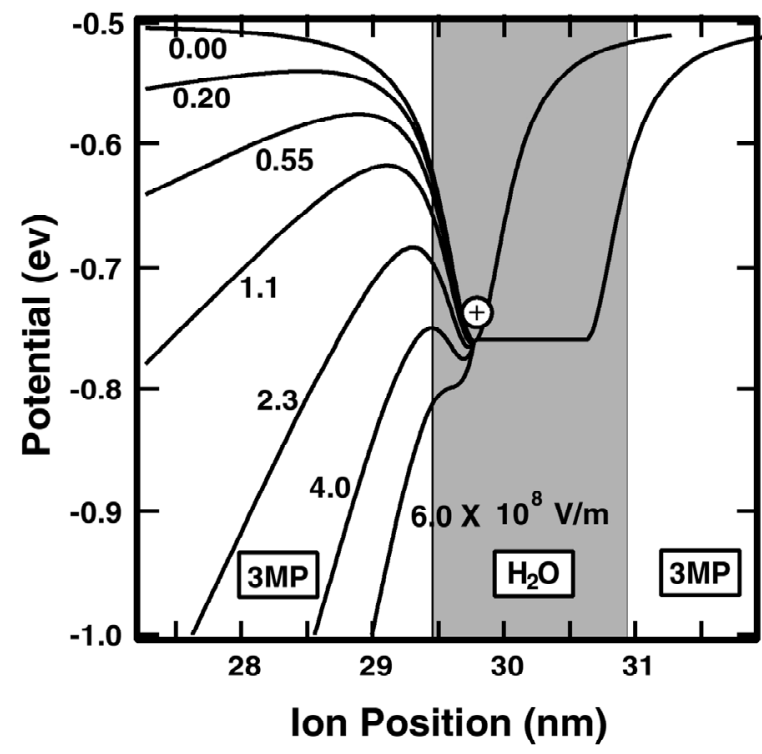

Figure 2.15. Solvation potential near water interface. A nanometer water film (shaded) is located between two organic films of 3-methylpentane, creating a solvation potential minimum at water layer. This is strongly altered by the electric field that ions can collectively create.

field (see Figure 2.17). At low field, the temperature must be higher for the ions to thermally escape the trap. At higher field, the ions can escape at a lower temperature. This gives a unique way to measure both the depth and shape of the solvation potential.

\section{Nano-Scale "Fast and Slow Lanes": The Interfacial Fluidity of 3-Methylpentane Films}

To address the fluidity of confined liquids, the nano-scale perturbations from bulk fluidity were measured near the interfaces of 3MP films, with a spatial resolution of $4 \AA$. Using ion mobility to probe the spatially varying flow properties, it is observed that films of glassy 3MP are much less viscous at the vacuum-liquid interface and more viscous at the 3MP-metal interface, compared to the bulk film. The apparent decrease in viscosity near the vacuum-interface at $85 \mathrm{~K}$ is found to be six orders of magnitude. The fluidity perturbations persist about $3 \mathrm{~nm}$ into the film.

Fluids in "confined spaces" (typically nanometersized) are increasingly seen as having properties that can differ markedly from that of the bulk fluid. This can be profoundly important as nanometer fluids are common in biological systems (e.g. ion channels), in heterogeneous chemistry (e.g. nanometer powders or zeolite-like structures) 
or in some nanoscale devices being dreamt up in labs across the country. Even a bulk fluid has interfaces, and if the properties of the fluid are strongly altered in the nanometer region of the interface, this could dramatically alter the fluid's success as a lubricant or adhesive, or in interfacial transfer kinetics. Properties commonly believed to be altered at the interfaces of a fluid are the viscosity, molecular density, and ordering (crystallinity).

One issue in confined liquids is whether the properties change continuously, as one moves away from the interface, or whether the interfaces cause a uniform change across the confined liquid. If the changes are continuous, many observations could be pictured as analogous to traffic flow on a highway. Interfaces that increased the fluidity would generate a kinetic "fast lane" adjacent to it, while an interface that decreased the viscosity of the adjoining fluid would create a "slow lane." In either case, one would have normal flow properties farther from the interface. Or do the interfaces uniformly change the overall "speed limit"?

Certainly this will depend on the distance scale of the confined liquids and probes used. Most researchers agree that the effects are likely to be strongest within a few nanometers of the interface, beyond the resolution of most techniques. We devised a non-perturbative probe of the interfacial fluidity near the free and solid interfaces of nanometer fluid films with $4 \AA$ fluidity resolution. Our experiment is similar to that shown in Figures 2.16 and 2.17 (but without any water film). A 3MP layer is epitaxially deposited with submonolayer accuracy using a molecular beam onto a $\operatorname{Pt}(111)$ substrate maintained at less than $30 \mathrm{~K}$. This is below its $T_{g}$ of $77 \mathrm{~K}$, so no fluid motion is possible. Next, $\mathrm{D}_{3} \mathrm{O}^{+}$ions are gently deposited on the $30 \mathrm{~K}$ film with a kinetic energy of approximately $1 \mathrm{eV}$. In many of the experiments, a final step was to deposit an additional 3MP film on top of the ions. The ions on or within the insulating film create a capacitor. The potential difference across the film; $\Delta \mathrm{V}$ is proportional to the average height of the ions in the film $\Delta \mathrm{V}$ can be measured to $10 \mathrm{mV}$ precision with a non-contact Kelvin probe. Thus we have a very sensitive probe of the ion motion within our sculpted films. In our

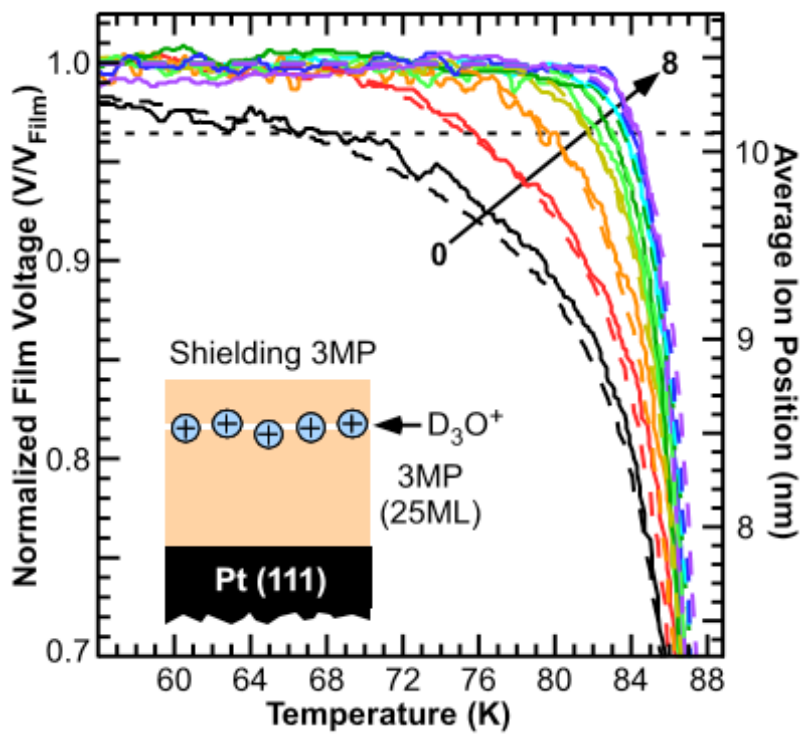

Figure 2.16. Fast ion motion near free interface. A slowly heated sandwich of 3MP (n monolayers/ ions/3MP (25 monolayers) on a Pt(111) substrate is monitored for ion motion as it passes the glass $\mathrm{T}$ of $77 \mathrm{~K}$. As the topmost layer of $3 \mathrm{MP}$ goes from $\mathrm{n}=0$ to 8 monolayer, the ions change from moving at much lower $T$ than predicted by the bulk viscosity to moving as expected for the bulk viscosity. Dashed curves are as expected for surface-perturbed viscosity.

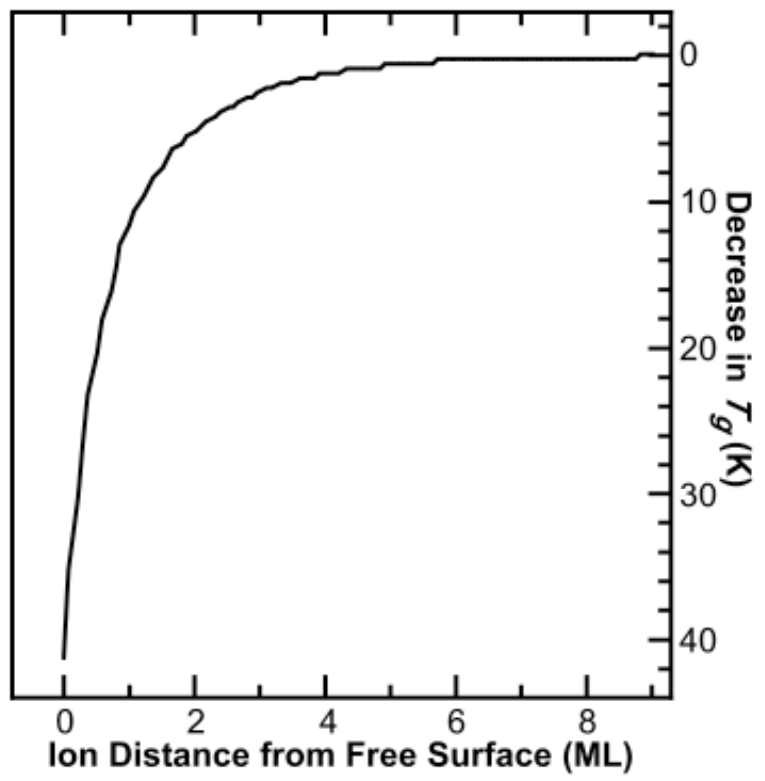

Figure 2.17. Glass temperature changes near the free interface. Shown is the inferred change in glass temperature of 3-methlypentane as a function of distance from the free (vacuum) interface. 
experiments, we report the ion motion (via the Kelvin probe) as the composite film is warmed slowly at $0.2 \mathrm{~K} / \mathrm{s}$.

The transport of $\mathrm{D}_{3} \mathrm{O}^{+}$ions through a $25 \mathrm{ML}$ $(10.5 \mathrm{~nm}) 3 \mathrm{MP}$ film is shown as the bottom-most solid curve in Figure 2.16. As the temperature of the film is increased, the ions move quickly through the film in the range of 80 to $95 \mathrm{~K}$ as the viscosity of the $3 \mathrm{MP}$ rapidly decreases. This is evidenced by a drop in the film voltage.

If one estimates how the ions should move using the properties of bulk 3MP, the right-most dashed curve is predicted. The severe discrepancies indicate that the vacuum interface causes a loosening of the surface layers (we also see a stiffening of the $3 \mathrm{MP}$ at the $\mathrm{Pt}(111)$ interface).

This surface-enhanced fluidity can be explored by initially and precisely placing the ions at increasing distances from the free interface. This is demonstrated in Figure 2.16 for 3MP films where the ions are at a constant initial height of $25 \mathrm{ML}$ from the Pt as increasing layers of $3 \mathrm{MP}$ are placed on top of the ions, shielding them from the interface.

The bottom curve is for a film with no shielding layers. As the number of shielding layers of 3MP is increased, the mobility of the ions is observed to approach that expected for a position-independent bulk film viscosity. Finally, with the ions $7 \mathrm{ML}(2.9 \mathrm{~nm})$ or more away from the interface, the mobility is extremely close to that predicted using the bulk film viscosity.

To numerically parameterize the interfacial effects on fluidity, we modeled it as if $T_{g}$ was a function of distance from the top. Using the distance dependence shown in Figure 2.17, we get a good fit to the observed ion motion (dashed curves in Figure 2.16). At the Pt interface, similar studies by us show a stiffening compared to the bulk, that also persists over about 7 ML.

Our studies clearly indicate that ion mobility in thin $3 \mathrm{MP}$ films change dramatically near the interfaces demonstrating both the fast and slow lanes, over a length scale of about 7 monolayers $(3 \mathrm{~nm})$ at the free and Pt-3MP interfaces, respectively. We hope in the future this may open up opportunities to improve nano-devices or lubrication.

\section{Nitric Acid-Water Complexes: Theoretical Calculations and Comparison to Experiment}

\author{
P. R. McCurdy, ${ }^{(a)}$ W. P. Hess, \\ and S. S. Xantheas
}

Supported by DOE Office of Basic Energy Sciences, Chemical Sciences Division.

(a) LSI Logic, Portland, Oregon.

Nitric acid and its hydrates play and important role in atmospheric chemistry (Hamil and Toon 1991). In the stratosphere, nitric acid hydrates are the primary components of the type I polar stratospheric clouds - a key player in the seasonal depletion of polar ozone. Alternatively, the relative stability of $\mathrm{HNO}_{3}$ allows transport to the troposphere where it gives rise to acid rain. The macroscopic properties of the binary nitric acid-water system are an essential element of a multi-component thermodynamic model that describes atmospheric processes. Ritzhaupt and Devlin (1973) have previously studied $\mathrm{HNO}_{3} \cdot \mathrm{H}_{2} \mathrm{O}$ complexes in Ar matrices using FTIR spectroscopy. They concluded that at low concentrations of water in the matrix $(<3 \%) \mathrm{HNO}_{3} \cdot \mathrm{H}_{2} \mathrm{O}$ exists primarily as a molecular complex, and above this concentration the solvated ion pair becomes important. A significant splitting in the asymmetric stretch $\left(\Delta v_{3}\right)$ of the $\mathrm{NO}_{3}{ }^{-}$ion was noted that varied with the degree of solvation. In a later study of solid $\mathrm{H}_{2} \mathrm{O}-\mathrm{HNO}_{3}$ mixtures, the same authors suggested as many as three water molecules may be required to ionize the $\mathrm{HNO}_{3}$ molecule (Ritzhaupt and Devlin, 1991). However, no definitive conclusions were drawn, and no direct evidence for the existence of higher order molecular species other than the 1:1 complex was presented. Barnes, et al. (1994) have also previously studied $\mathrm{HNO}_{3} \cdot \mathrm{H}_{2} \mathrm{O}$ complexes in Ar matrices using FTIR spectroscopy. Their study focused on the $\mathrm{H}-\mathrm{ONO}_{2}$ stretching region of the complex in 1:1:200 $\mathrm{HNO}_{3}: \mathrm{H}_{2} \mathrm{O}:$ Ar matrices. 
In the present study we investigate the formation of $\mathrm{HNO}_{3} \cdot\left(\mathrm{H}_{2} \mathrm{O}\right)_{\mathrm{n}}$ complexes from first principles electronic structure methods and compare our findings to new and previously reported experimental results. The calculations yielded optimized ground state structures and harmonic vibrational frequencies for the $\mathrm{HNO}_{3} \cdot\left(\mathrm{H}_{2} \mathrm{O}\right)_{\mathrm{n}}(n=0-5)$ clusters and their $\mathrm{DNO}_{3}$ analogs. We also present new FTIR data and discuss spectral assignments in light of the new theoretical results. The calculated results aid in the interpretation of the matrix isolated FTIR spectra indicating that dissociation occurs for cluster sizes greater than $n=4$.

Experiments were conducted using a matrixisolation FTIR apparatus described previously (Rowland and Hess, 1997). Argon solutions of $\mathrm{HNO}_{3}$ and $\mathrm{H}_{2} \mathrm{O}$ or their deuterated analogs $\left(\mathrm{DNO}_{3}\right.$ and $\mathrm{D}_{2} \mathrm{O}$ ) were co-deposited from separate nozzles onto $\mathrm{KBr}$ substrates cooled to $12 \mathrm{~K}$ by a closed-cycle helium cryostat. For annealed samples, FTIR spectra were taken at $12 \mathrm{~K}$ after annealing for 5 minutes at the indicated temperature. Thin films were vapor deposited to a thickness of approximately $40 \mu \mathrm{m}$ over a period of approximately 2 hours. Thin film growth was monitored by recording the interference fringes of a reflected helium-neon laser beam aligned at a near-normal incidence to the substrate. FTIR spectra of condensed samples were collected for 256 scans at a resolution of $1 \mathrm{~cm}^{-1}$. The spectra of $\mathrm{HNO}_{3}: \mathrm{H}_{2} \mathrm{O}: \mathrm{Ar}$ and $\mathrm{DNO}_{3}: \mathrm{H}_{2} \mathrm{O}:$ Ar matrices were obtained at various dilution factors and compared with equivalent $\mathrm{HNO}_{3}$ : Ar and $\mathrm{H}_{2} \mathrm{O}$ :Ar mixtures. Spectra of $\mathrm{HNO}_{3}: \mathrm{H}_{2} \mathrm{O}$ :Ar matrices show several distinct features that do not correlate to either $\mathrm{HNO}_{3}$ or $\mathrm{H}_{2} \mathrm{O}$ species and, therefore, are assigned to nitric acid-water complexes.

Figure 2.18 displays the spectra from $\mathrm{DNO}_{3}: \mathrm{H}_{2} \mathrm{O}:$ Ar matrices between 1900 and $2650 \mathrm{~cm}^{-1}$, a region relatively free of interference due to overlapping bands. The O-D stretching bands associated with $\mathrm{DNO}_{3}\left(\mathrm{H}_{2} \mathrm{O}\right)_{n}$ clusters up to $n=3$ are apparent as the water concentration is increased. The strongest feature in the low water concentration matrix $(1: 1: 200)$ is the O-D stretch of the $\mathrm{DNO}_{3}$ monomer at $2601 \mathrm{~cm}^{-1}$ seen in Figure 2.18(a). In the higher water concentration spectrum, two strong bands centered at 2292 and

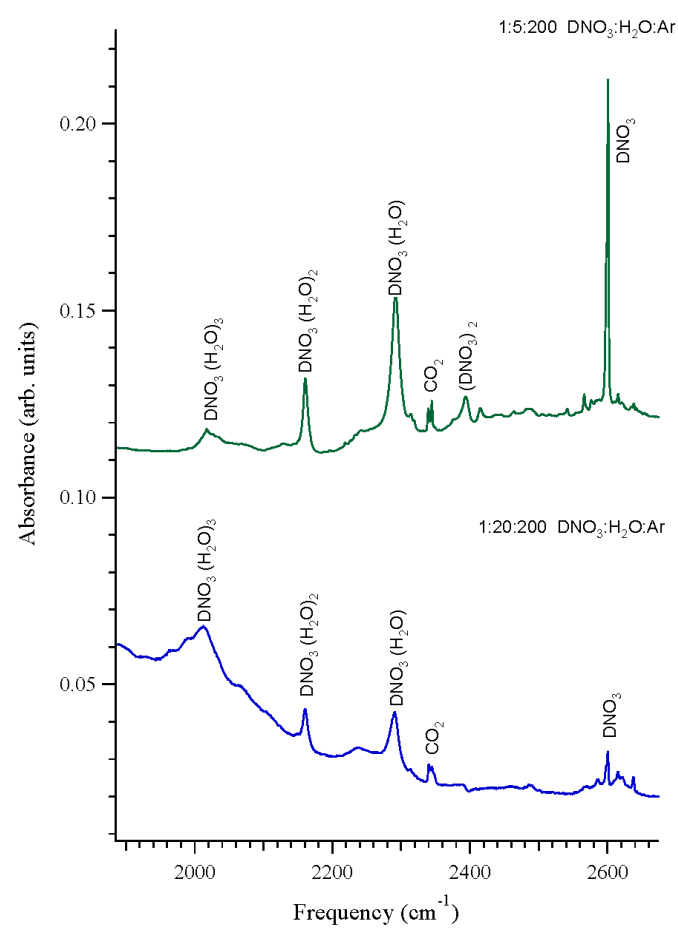

a)

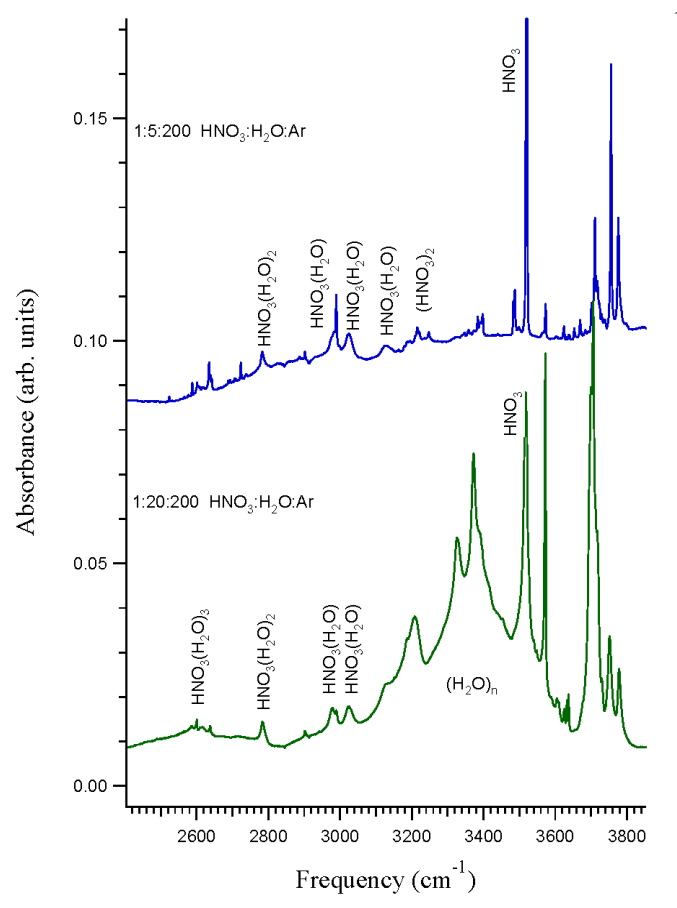

b)

Figure 2.18. FTIR absorption spectra of the O-D, and $\mathrm{O}-\mathrm{H}$ stretch bands for (a) DNO3:H2O:Ar matrices and (b) HNO3:H2O:Ar matrices at 1:5:200 (Upper trace), and 1:20:200 (lower trace) gas precursor ratios. 
$2161 \mathrm{~cm}^{-1}$ are evident, as is a strong broad feature near $2017 \mathrm{~cm}^{-1}$. These bands are shifted from the $\mathrm{DNO}_{3}$ monomer O-D stretch frequency by -309 , -440 and $-586 \mathrm{~cm}^{-1}$, respectively, which compares very well with the calculated harmonic shifts of $-299,-461$, and $-575 \mathrm{~cm}^{-1}$ for the $\mathrm{DNO}_{3}\left(\mathrm{H}_{2} \mathrm{O}\right)_{n}$ clusters, $n=1,2,3$. The integrated band intensity of the isolated $\mathrm{DNO}_{3} \mathrm{O}-\mathrm{D}$ stretch feature decreases sharply in the high water concentration spectrum indicating that nearly all $\mathrm{DNO}_{3}$ forms a complex with water. The integrated band intensities of the features at 2292 and $2161 \mathrm{~cm}^{-1}$ also decrease with increasing water concentration, while the broad feature at $2017 \mathrm{~cm}^{-1}$ increases.

Figure 2.18(b) displays matrix spectra of $\mathrm{HNO}_{3}$, and $\mathrm{HNO}_{3}\left(\mathrm{H}_{2} \mathrm{O}\right)_{n}$ clusters in the $\mathrm{H}-\mathrm{ONO}_{2}$ stretch region between 2400 and $3850 \mathrm{~cm}^{-1}$. Unlike the $\mathrm{DNO}_{3}$ spectra of Figure 2.18(a), the $\mathrm{H}-\mathrm{ONO}_{2}$ stretch region suffers from interference due to overlapping bands of water and larger $\left(\mathrm{H}_{2} \mathrm{O}\right)_{n}$ clusters. The $\mathrm{O}-\mathrm{H}$ stretching bands associated with $\mathrm{HNO}_{3}\left(\mathrm{H}_{2} \mathrm{O}\right)_{n}$ clusters up to $n=3$ can nonetheless be identified. The strongest feature in the 1:5:200 matrix is again the (offscale) $\mathrm{O}-\mathrm{H}$ stretch fundamental of the nitric acid monomer. In the region where the two $\mathrm{OH}$ stretches of the $\mathrm{HNO}_{3}\left(\mathrm{H}_{2} \mathrm{O}\right)$ and $\mathrm{HNO}_{3}\left(\mathrm{H}_{2} \mathrm{O}\right)_{2}$ complexes are expected, we observe four bands. These four bands, centered at 2784, 2981, 3024, and $3131 \mathrm{~cm}^{-1}$, were assigned by Barnes et al. as arising from the $\mathrm{HNO}_{3}\left(\mathrm{H}_{2} \mathrm{O}\right)$ complex. Three of the bands were attributed to "Fermi resonances" with overtones and combination bands: $2 v_{4}$ at $2784 \mathrm{~cm}^{-1} ;\left(v_{2}+v_{3}\right)$ at $2981 \mathrm{~cm}^{-1}$; and $\left(v_{2}+v_{4}\right)$ at $3130 \mathrm{~cm}^{-1}$. The remaining band at $3024 \mathrm{~cm}^{-1}$ was assigned to the $v_{1} \mathrm{O}-\mathrm{H}$ stretch of the $\mathrm{HNO}_{3}\left(\mathrm{H}_{2} \mathrm{O}\right)$ complex.

Figure 2.19(a) shows the FTIR spectra of the DO- $\mathrm{NO}_{2}$ stretch region for $\mathrm{DNO}_{3}: \mathrm{H}_{2} \mathrm{O}$ :Ar matrices as a function of increasing $\mathrm{H}_{2} \mathrm{O}$ ratio. The prominent peak at $887 \mathrm{~cm}^{-1}$ in the upper trace is assigned to $v_{5}$, the DO- $\mathrm{NO}_{2}$ stretch of the monomer, while the peak at $1013 \mathrm{~cm}^{-1}$ is due to the $\mathrm{D}-\mathrm{O}-\mathrm{NO}_{2}$ bending mode. As expected, these peaks decrease in intensity as the concentration of $\mathrm{H}_{2} \mathrm{O}$ in the matrix is increased, and the spectra displays features associated with hydrated $\mathrm{DNO}_{3}$ species. The top trace of Figure 2.19(a) shows a series of peaks between 920 and $950 \mathrm{~cm}^{-1}$. The
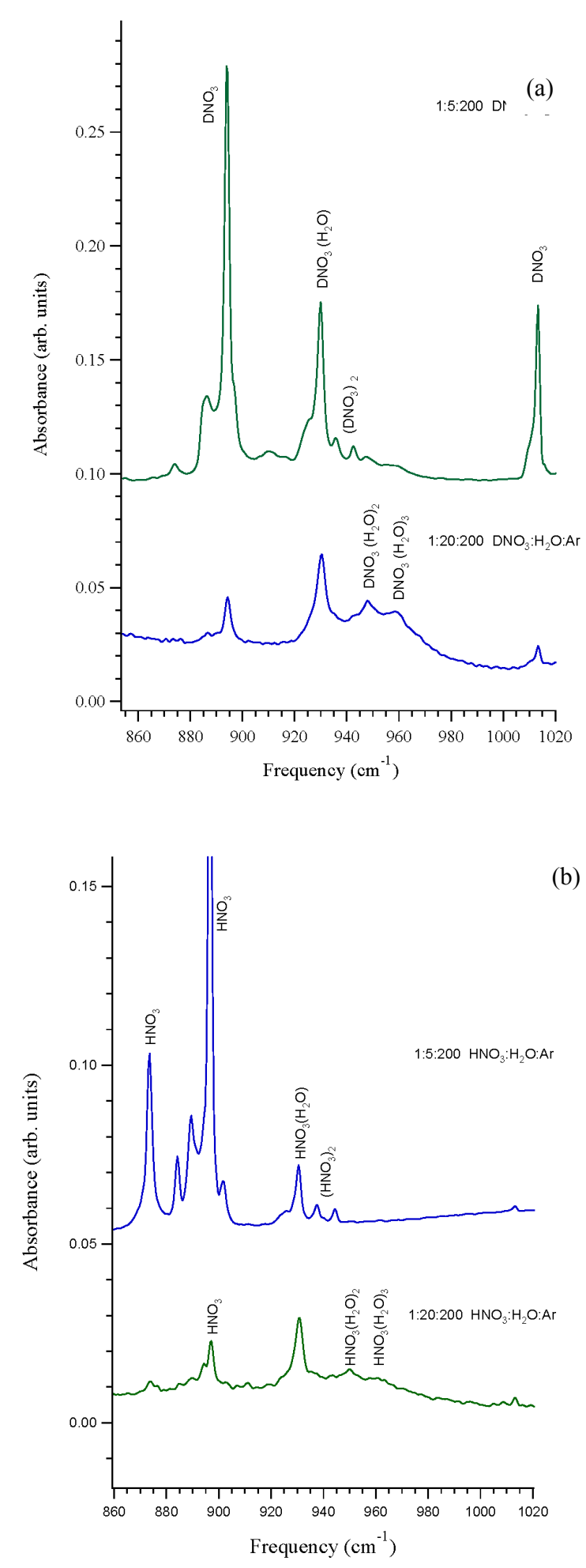

Figure 2.19. FTIR absorption spectra of the O-N, stretch bands for (a) DNO3:H2O:Ar matrices and (b) HNO3:H2O:Ar matrices at 1:5:200 (upper trace), and 1:20:200 (lower trace) gas precursor ratios. 
sharp band centered at $930 \mathrm{~cm}^{-1}$ is present only in mixtures containing both $\mathrm{DNO}_{3}$ and $\mathrm{H}_{2} \mathrm{O}$ and is assigned to the $\mathrm{DNO}_{3} \cdot \mathrm{H}_{2} \mathrm{O}$ complex. Two additional broad weak bands centered at 948 and $960 \mathrm{~cm}^{-1}$ are apparent. We assign these two peaks to the $\mathrm{DNO}_{3} \cdot\left(\mathrm{H}_{2} \mathrm{O}\right)_{2}$ and $\mathrm{DNO}_{3} \cdot\left(\mathrm{H}_{2} \mathrm{O}\right)_{3}$ complexes. The relative integrated absorption of the 948 and $960 \mathrm{~cm}^{-1}$ bands is greatest in the 1:20:200 sample.

The frequency shifts calculated for the $\mathrm{D}-\mathrm{ONO}_{2}$ $\left(\mathrm{H}_{2} \mathrm{O}\right)_{n}$ clusters $(n=1-3)$ show quantitative agreement with the measured FTIR data. In particular, the O-D stretch spectral region displays brands separated by frequency intervals rather accurately predicted by our calculations. Qualitative agreement is also found for the $\mathrm{O}-\mathrm{H}$ spectral region of the $\mathrm{HNO}_{3}\left(\mathrm{H}_{2} \mathrm{O}\right)_{n}$ system and the N-OD stretch and $\mathrm{N}-\mathrm{O}-\mathrm{D}$ bend spectral regions of the $\mathrm{DNO}^{3}\left(\mathrm{H}_{2} \mathrm{O}\right)_{n}$ system. ON the strength of the correspondence between the calculated and experimental results, we may tentatively assign the measured bands for the nitric acid-water clusters up to $n=3$.

We regard our band assignments as tentative due to some ambiguities apparent in the experimental results and discrepancies with the interpretations of results published by other researchers. The most likely cause for the discrepancies is the effect of the argon matrix environment. The formation of clusters within the argon matrix cannot be controlled ideally by simply changing the deposition conditions. Furthermore, any particular cluster could occupy a variety of matrix environments or configurations. The exact effects of varying the deposition conditions and anneal history are not known. Since the cluster distribution is unknown, we can only expect integrated band intensities to reflect differences in growth conditions in a qualitative fashion. Therefore, we tentatively assign the band centered at $2784 \mathrm{~cm}^{-1}$ to the $\mathrm{HNO}_{3} \cdot\left(\mathrm{H}_{2} \mathrm{O}\right)_{2}$ complex and the band centered at $2613 \mathrm{~cm}^{-1}$ to the $\mathrm{HNO}_{3} \cdot\left(\mathrm{H}_{2} \mathrm{O}\right)_{3}$ complex. This latter band displays the expected increase in integrated intensity with water concentration. This assignment is further strengthened by the qualitative agreement of the ab initio results with the observed spectral features for several vibrational modes of the $n=2$ and 3 clusters.
Figure 2.18(a) shows two peaks $\left(2292 \mathrm{~cm}^{-1}\right.$ and $2161 \mathrm{~cm}^{-1}$ ) in the region where the OD stretch of the $\mathrm{DNO}_{3} \cdot \mathrm{H}_{2} \mathrm{O}$ complex is expected. The ratio of their integrated intensities as a function of $\mathrm{H}_{2} \mathrm{O}$ concentration is nearly constant suggesting they could be derived from the same species. While it is clear that the band centered at $2292 \mathrm{~cm}^{-1}$ is due to the O-D stretch of the $\mathrm{DNO}_{3} \cdot \mathrm{H}_{2} \mathrm{O}$ complex, the second band is shifted by $-440 \mathrm{~cm}^{-1}$ from O-D stretch of the isolated monomer which compares remarkably well to the calculated shift $\left(-462 \mathrm{~cm}^{-1}\right)$ in the $\mathrm{DNO}_{3}\left(\mathrm{H}_{2} \mathrm{O}\right)_{2}$ complex. The assignment of this band to the $\mathrm{DNO}_{3}\left(\mathrm{H}_{2} \mathrm{O}\right)_{2}$ complex is also supported by the similar behavior of the band assigned to the $\mathrm{DNO}_{3}\left(\mathrm{H}_{2} \mathrm{O}\right)_{2}$ complex for the DO- $\mathrm{NO}_{2}$ stretch which is shifted by $60 \mathrm{~cm}^{-1}$.

The $\mathrm{HNO}_{3} \cdot \mathrm{H}_{2} \mathrm{O}$ complex actually shows four peaks separated by more than $300 \mathrm{~cm}^{-1}$ in the $\mathrm{OH}$ stretch region. The fact that only two analogous peaks are observed in the corresponding portion of the FTIR spectrum for the $\mathrm{DNO}_{3} / \mathrm{H}_{2} \mathrm{O} / \mathrm{Ar}$ matrix support the "Fermi resonance" explanation for at least two of the peaks seen in the $\mathrm{HNO}_{3} / \mathrm{H}_{2} \mathrm{O} / \mathrm{Ar}$ spectrum. Still, no combination of frequencies for the $\mathrm{DNO}_{3} \cdot \mathrm{H}_{2} \mathrm{O}$ complex match the observed frequency and hence we assign the band centered at $2162 \mathrm{~cm}^{-1}$ to the $\mathrm{DNO}_{3}\left(\mathrm{H}_{2} \mathrm{O}\right)_{2}$ complex. Figure 2.18(a) also shows a broad peak at $\sim 2015 \mathrm{~cm}^{-1}$. This peak is not observed in very low water concentration spectra (not shown here) but is clearly observed in the 1:5:200 spectrum. The integrated absorption intensity of the band centered at $2015 \mathrm{~cm}^{-1}$ increases with water concentration and the band broadens further. We assign this band to the $\mathrm{DNO}_{3} \cdot\left(\mathrm{H}_{2} \mathrm{O}\right)_{3}$ complex.

\section{References}

Barnes, A. J., E. Lasson, and C. J. Nielson, J. Mol. Struct. 322, 165 (1994).

Hamill, P. and O. B. Toon, Physics Today, December issue, 34 (1991).

Ritzhaupt, G. and J. P. Devlin, J. Phys. Chem. 77, 57 (1973).

Ritzhaupt, G. and J. P. Devlin, J. Phys. Chem. 95, 90 (1991).

Rowland, B. and W. P. Hess, J. Phys. Chem. A 101, 8049 (1997). 


\section{High-Energy Processes at Environmental Interfaces}

This research area focuses on obtaining a mechanistic understanding of chemical transformations resulting from electronic excitation in condensed phase materials relevant to the DOE environmental clean-up mission. Energetic processes are important in the degradation of mixed wastes due to radiolytic decay driven chemistry occurring in solid and liquid phases of stored tank waste and final waste forms. Reactions occurring at interfaces are of particular interest for characterizing material composition and response to electronic excitation. We use pulsed femtosecond and nanosecond laser sources to study laser-solid interactions in a combined experimental and theoretical program. Our goal is to develop models for excited state reactions by measuring ultrafast dynamic processes and by demonstrating laser control of solid-state chemistry. 


\section{Control of Laser Desorption Using Tunable Single Pulses and Pulse Pairs}

\author{
W. P. Hess, ${ }^{(a)}$ A. G. Joly, ${ }^{(a)}$ \\ D. P. Gerrity, ${ }^{(b)}$ K. M. Beck, ${ }^{(a)}$ \\ P. V. Sushko, ${ }^{(c}$, and A. L. Shluger ${ }^{(c)}$ \\ Supported by DOE Office of Basic Energy \\ Sciences, Chemical Sciences Division. \\ (a) Pacific Northwest National Laboratory. \\ (b) Department of Chemistry, Reed College. \\ (c) Department of Physics and Astronomy, \\ University College London.
}

Irradiation of insulating materials by relatively low energy electrons, $\gamma-, \mathrm{X}$-, synchrotron and UV-photons can induce surface decomposition and emission of particles in different charge and electronic states and with different velocity distributions. This irradiation is mostly absorbed inside the solid, and only a minor part of it is manifested in particle desorption. A more gentle approach is used in photo-induced desorption of individual surface atoms (Kanaski et al. 1998) specifically targeting surface chemical bonds. Recently lasers and other UV photon sources (Zema et al. 1997; Hess et al. 2001) have been used to selectively excite particular surface features and to study the conversion of electronic excitation energy into surface decomposition, molecular dissociation, and formation of chemically active surface sites.

An issue key to all of these processes concerns the possibility of active control of the yield and properties of product species. Recent experiments on laser induced desorption of alkali halides (Zema et al. 1997; Hess et al. 2001; Beck et al. 2001) have stimulated studies of laser control of these processes. In particular, it has been shown (Hess et al. 2001; Beck et al. 2001) that, by carefully choosing the excitation energy, it is possible to induce preferentially one mechanism of atom desorption and achieve selectivity in both kinetic energy and electronic state of desorbing bromine atoms from $\mathrm{KBr}$ (either the ground state $\mathrm{Br}\left[{ }^{2} \mathrm{P}_{3 / 2}\right]$ henceforth $\mathrm{Br}$, or the spin orbit excited state $\operatorname{Br}\left[{ }^{2} \mathrm{P}_{1 / 2}\right]$, henceforth $\left.\mathrm{Br}^{*}\right)$. We demonstrated that the velocity and the electronic state distributions of desorbing $\mathrm{Br}$ atoms strongly depend on photon energy.
On the basis of our recent measurements and theoretical calculations, we assume that single photons of 5.5 to $6.5 \mathrm{eV}$ energy can selectively excite surface or near-surface excitons leading to almost exclusive desorption of the hyper-thermal $\mathrm{Br}$ atoms (Hess et al. 2001; Beck et al. 2001). Another approach exploits a pump-pump scenario widely used in coherent control experiments. Generally speaking, the second pulse can be used to excite some of the relatively long-lived transient species produced near the surface by the first pulse. The photon energy selective approach takes advantage of energetic differences between surface and bulk exciton states and probes directly the surface exciton. The two-pulse approach relies upon production and manipulation of transient species within the crystal and near the surface. Each of these approaches can be used to control the yield and properties of desorbed $\mathrm{Br}$ atoms.

We irradiate the $\mathrm{KBr}$ surface at room temperature using nanosecond laser pulses and pulse combinations. Irradiation of the $\mathrm{KBr}$ surface induces emission of fast bromine atoms and thermal potassium atoms. The desorbed atoms are detected using laser ionization combined with time-of-flight (TOF) mass spectrometry. Velocity profiles reflecting the velocity distributions of photo-desorbed atoms are determined by integrating the atom yield as a function of the delay between excitation and probe lasers. The velocity profiles may be converted to kinetic energy distributions by applying the appropriate Jacobian transform (Beck et al. 2001). The single-pulse excitation experiments use stimulated Raman shifting of $\mathrm{Nd}$ :YAG laser harmonics (in hydrogen gas) to generate the necessary photon energies. The pulse pair experiments use the $266 \mathrm{~nm} \mathrm{Nd:YAG} \mathrm{fourth} \mathrm{harmonic}$ directly to create transient defect centers at the surface and within the bulk crystal (pump pulse 1). The second excitation pulse (pump pulse 2, the Nd:YAG third harmonic at $355 \mathrm{~nm}$ ) is delayed by $20 \mathrm{~ns}$ and overlapped spatially with pump pulse 1 . Pump pulse 2 excites transient defect centers and induces bromine emission. In all experiments, tunable light from a $\mathrm{Nd}$ :YAG pumped frequency-doubled dye laser, operating at $20 \mathrm{~Hz}$, is used to ionize $\mathrm{Br}$ and $\mathrm{Br}$ * atoms in a $(2+1)$ resonance-enhanced multiphoton ionization scheme. 
Figure 3.1 displays the $\mathrm{Br}$ atom kinetic energy distributions for photon energies between 5.5 and $6.5 \mathrm{eV}$. The photon energy range used overlaps the long wavelength edge of the $\mathrm{KBr}$ bulk absorption band (Urbach tail). The kinetic energy distributions change markedly with photon energy. The peak kinetic energies of desorbed $\mathrm{Br}$ are $0.37,0.24,0.18$ and $0.12 \mathrm{eV}$ using pump photon energies of 6.46, 6.07, 5.94, and $5.56 \mathrm{eV}$, respectively. Our results clearly demonstrate that one can achieve active incoherent control of the properties of photo-desorbed $\mathrm{Br}$ atoms from the $\mathrm{KBr}$ (001) surface as the velocity of $\mathrm{Br}$ atoms photodesorbed from the $\mathrm{KBr}$ crystal can be controlled using tunable laser light near the UV absorption threshold.

Figure 3.2 displays kinetic energy distributions of desorbed $\mathrm{Br}$ and $\mathrm{Br}^{*}$ atoms following sequential excitation by $4.7 \mathrm{eV}(266 \mathrm{~nm})$ and $3.5 \mathrm{eV}(355 \mathrm{~nm})$ nanosecond laser pulses. The energy distribution for both spin states is remarkably similar. The peak kinetic energy is roughly $0.12 \mathrm{eV}$; this is well above that expected for thermal desorption but generally below that obtained following resonant one-photon excitation. The relative $\mathrm{Br} / \mathrm{Br}^{*}$ product yield ratio resulting from two-laser induced emission (4.7 and $3.5 \mathrm{eV}$ ) is $1.4 \pm 0.6$. The relative $\mathrm{Br}^{*}$ yield is

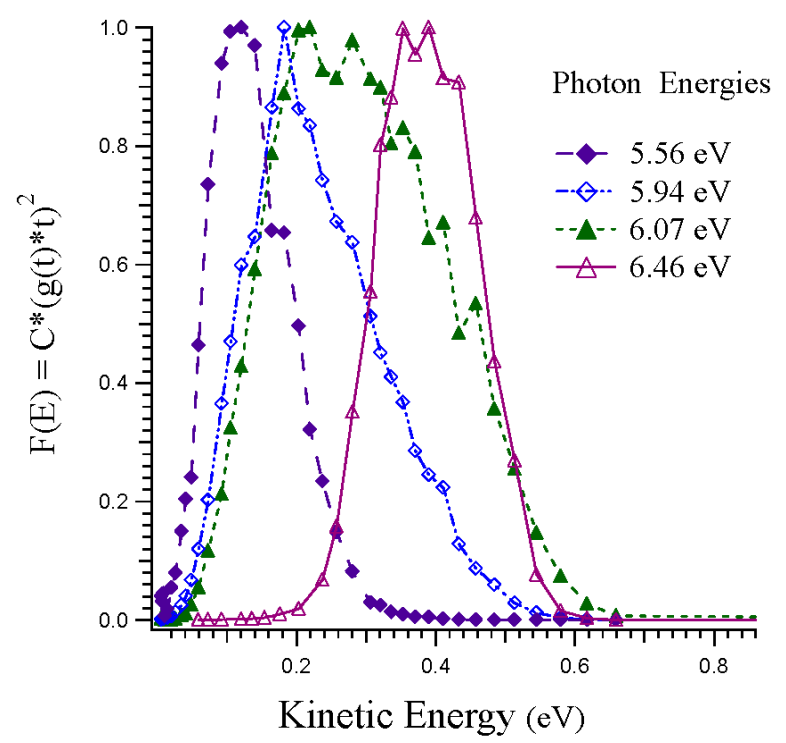

Figure 3.1. Kinetic energy distributions of $\mathrm{Br}$ emission following tunable band edge excitation. The peak of the $\mathrm{Br}$ kinetic energy distributions shift as photon energy is decreased. The solid and dashed lines serve only to guide the eye.

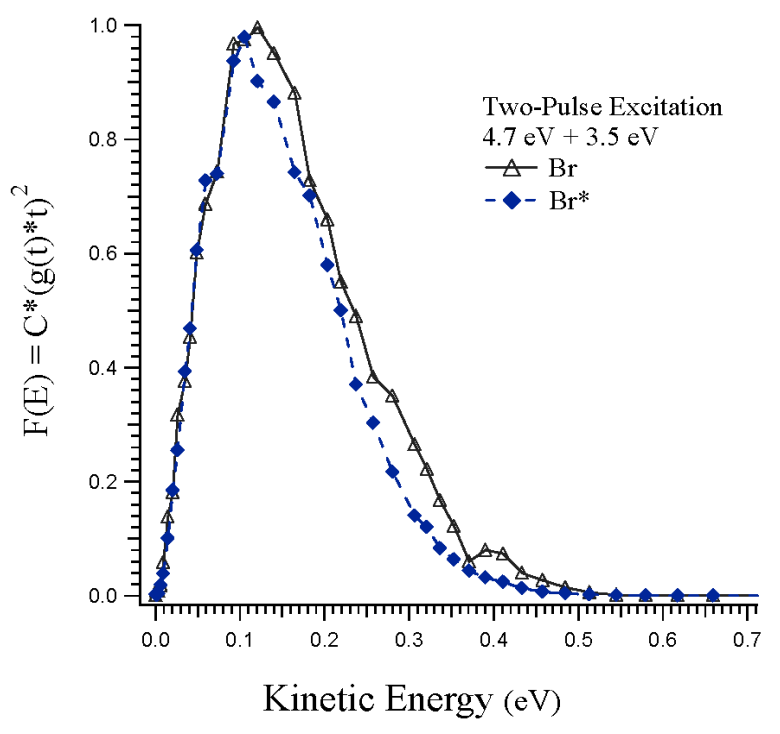

Figure 3.2. Kinetic energy distributions of $\mathrm{Br}$ and $\mathrm{Br}^{*}$ emission following 4.7 and delayed $3.5 \mathrm{eV}$ excitation. The $\mathrm{Br}$ (triangles) and $\mathrm{Br}^{*}$ (diamonds) display near identical kinetic energies. The solid and dashed lines serve only to guide the eye.

approximately 500 times greater than that obtained following resonant one laser excitation although the total atom yield is much lower, approaching only one percent of the $6.4 \mathrm{eV}$ yield. We have also produced $\mathrm{Br}$ and $\mathrm{Br} *$ emission using $6.4 \mathrm{eV}$ excitation followed by $3.5 \mathrm{eV}$ nanosecond laser pulses. $\mathrm{The} \mathrm{Br}$ and $\mathrm{Br}^{*}$ kinetic energy distributions produced by this latter pulse combination are identical, within error, to those displayed in Figure 3.2. However, when pump laser 2 is tuned to 4.7 or $2.3 \mathrm{eV}$ no significant increase in $\mathrm{Br}$ or $\mathrm{Br}^{*}$ emission is observed.

The relative yield of $\mathrm{Br}$ to $\mathrm{Br} *$ can be enhanced, over single photon resonant excitation, using a pulse-pair excitation scheme. Under the two-pulse $4.7+3.5 \mathrm{eV}$ excitation, the increase in the relative $\mathrm{Br}^{*}$ to $\mathrm{Br}$ ratio is roughly 500 times greater than the single laser result at $6.4 \mathrm{eV}$. The similar velocity profiles and halogen atom yield of the two spin states, displayed in Figure 3.2, suggests that both emissions result from excitation of the same transient center precursor. If we assume that the electronic excitation of $\mathrm{H}$ centers leads to dissociation or stimulates diffusion of $\mathrm{Br}$ and $\mathrm{Br}^{*}$, this mechanism could also explain the high $\mathrm{Br}^{*}$ yield following two-laser excitation. The $\mathrm{H}$ center is an interstitial defect and its diffusion takes place via the displacement sequence of $\mathrm{Br}$ atoms 
(Puchin et al. 1993). Near the surface this could easily bring the $\mathrm{H}$ center to the surface plane. Surface $\mathrm{H}$ centers are strongly polarized and represent a $\mathrm{Br}$ atom loosely adsorbed on a regular surface $\mathrm{Br}$ site. These $\mathrm{Br}$ atoms thermally desorb to produce the observed emission.

The $\mathrm{Br}$ and $\mathrm{Br}^{*}$ emission must be derived from a thin near-surface layer since the Br kinetic energy distribution would be relaxed to a thermal distribution if $\mathrm{Br}$ and $\mathrm{Br}^{*}$ were required to diffuse long distances through the bulk prior to emission. This conclusion is supported by the high $\mathrm{Br} *$ yield and the nearly identical $\mathrm{Br}$ and $\mathrm{Br}^{*}$ velocity profiles. Thus the $\mathrm{Br} / \mathrm{Br}^{*}$ ratio could be enhanced by using smaller time-delays between the first and second pulses which should allow one to excite more $\mathrm{H}$ centers. Since $\mathrm{H}$ centers are produced within a few ps of excitation (Song and Williams 1993; Shluger and Tanimura 2000), timeresolved experiments using femto-second pulses could shed more light on the dynamics of defect processes near the surface and mechanisms of desorption.

\section{References}

Beck, K. M., A. G. Joly, and W. P. Hess, Phys. Rev. B 63, 125423 (2001).

Hess, W. P., A. G. Joly, D. P. Gerrity, K. M. Beck, P. V. Sushko, and A. L. Shluger, J. Chem. Phys. 115, 9463 (2001).

Kanasaki, J., T. Ishida, K. Ishikawa, and K. Tanimura, Phys. Rev. Lett. 80, 4080 (1998).

Puchin, V. E., A. L. Shluger, and N. Itoh, Phys. Rev. B 47, 10760 (1993).

Puchin, V. E., A. L. Shluger, K. Tanimura, and N. Itoh, Phys. Rev. B 47, 6226 (1993).

Song, K. S. and R. T. Williams, Self-Trapped Excitons (Springer-Verlag, Berlin, 1993).

Shluger, A. L. and K. Tanimura, Phys. Rev. B 61, 5392 (2000).

Zema, N, M. Piacentini, P. Czuba, J. Kolodziej, P. Piatkowski, Z. Postawa, and M. Szymonski, Phys. Rev. B 55, 5448 (1997).

\section{Transient Center Photodecomposition in Potassium Bromide}

\author{
K. M. Beck ${ }^{(a)}$, A. G. Joly ${ }^{(a)}$, \\ W.P. Hess ${ }^{(a)}$, D. P. Gerrity ${ }^{(b)}$, \\ N. F. Dupuis ${ }^{(c)}$, P. V. Sushko ${ }^{(d)}$ and \\ A. L. Shluger ${ }^{(d)}$
}

Research supported by the Chemical Sciences Division of the DOE Office of Basic Energy Sciences.

(a) Pacific Northwest National Laboratory.

(b) Department of Chemistry, Reed College.

(c) School of Engineering and Applied Science, Columbia University.

(d) Condensed Matter and Materials Physics, Department of Physics and Astronomy, University College London.

\section{Introduction}

Recent experimental advances (Kolodziej et al. 1997; Zema et al. 1997; Such et al. 2000; Alexandrov et al. 2001; Beck et al. 2001; Hess et al. in press) and theoretical modeling (Hess et al. in press; Pachin et al. 1993) moved us closer to controlling desorption from alkali halides using laser pulses. We showed that, by carefully choosing the excitation energy, it is possible to induce preferentially one mechanism of atom desorption and achieve selectivity in both kinetic energy and electronic state of desorbing bromine atoms from $\mathrm{KBr}$ (Hess et al. In press) (either the ground state $\mathrm{Br}\left[{ }^{2} \mathrm{P}_{3 / 2}\right]$, henceforth $\mathrm{Br}$, or the spin orbit excited state $\operatorname{Br}\left[{ }^{2} \mathrm{P}_{1 / 2}\right]$, henceforth $\left.\mathrm{Br}^{*}\right)$. We have also demonstrated that the velocity distribution and the electronic state of desorbing $\mathrm{Br}$ atoms strongly depend on the photon energy. Excitation at $6.4 \mathrm{eV}$ led to desorption of almost exclusively hyper-thermal $\mathrm{Br}$ atoms whereas excitation at $7.9 \mathrm{eV}$ produced both thermal and hyper-thermal components. According to the theoretical model developed in references (Hess et al. in press; Pachin et al. 1993), the hyper-thermal component is caused by the decomposition of surface 
excitons into desorbing $\mathrm{Br}$ atoms and surface $\mathrm{F}$ centers. The thermal component results from decomposition of self-trapped excitons in the near-surface region into $\mathrm{F}$ (neutral halogen vacancy) and $\mathrm{H}$ (interstitial $\mathrm{Br}$ atom) centers and diffusion of their $\mathrm{H}$ centers to the surface, followed by thermally activated decomposition into $\mathrm{Br}^{-}$ions in regular surface sites and desorbed $\mathrm{Br}$ atoms. Our recent measurements and theoretical calculations demonstrated that $6.4 \mathrm{eV}$ photons resonantly excite surface excitons confirming the origin of the hyperthermal component (Hess et al. in press).

Further analysis of the photon-induced processes leading to desorption of $\mathrm{Br}$ atoms (Hess et al. in press) demonstrated a more complex interplay of several factors. In particular, the ratio of the thermal and hyperthermal components of desorption is determined by the relation between the bulk and surface excitation and the rate of recombination and aggregation of $F$ and $\mathrm{H}$ centers. To gain a deeper understanding of these factors, we compare desorption induced by nanosecond single-photon $6.4 \mathrm{eV}$ irradiation, which preferentially excite surface excitons, with desorption induced by femtosecond $3.2 \mathrm{eV}$ laser pulses. The latter is expected to excite more bulk excitons and hence create more thermal desorption. As desorption is strongly affected by the photon energy, we check the effect of a relatively small increase of the photon energy from $6.4 \mathrm{eV}$ to $7.0 \mathrm{eV}$. The latter is higher than the maximum of the bulk exciton absorption, about $6.55 \mathrm{eV}$ at $300 \mathrm{~K}$ (Tomiki et al. 1974), but lower than the band gap (about 7.4 eV). Again, we compare the $7.0 \mathrm{eV}$ excitation with that using $3.5 \mathrm{eV}$ photons. Yet another issue concerns the fact that $\mathrm{H}$ centers can be electronically excited by $3.2 \mathrm{eV}$ and $3.5 \mathrm{eV}$ photons. If significant, this absorption should affect the power dependence of our measurements and show up as a desorption signature.

\section{Results and Discussion}

Figure 3.3 displays the velocity profiles for $\mathrm{Br}$ following $3.2 \mathrm{eV}$ femto-second excitation and $6.4 \mathrm{eV}$ nano-second excitation. Excitation at $6.4 \mathrm{eV}$ is resonant with absorption of the surface exciton and a nearly pure hyper-thermal emission component is observed (Beck et al. 2001; Hess et al. in press). Because of the significantly higher irradiance, the femtosecond pulses are likely to cause two-photon absorption. Although both signals are dominated by

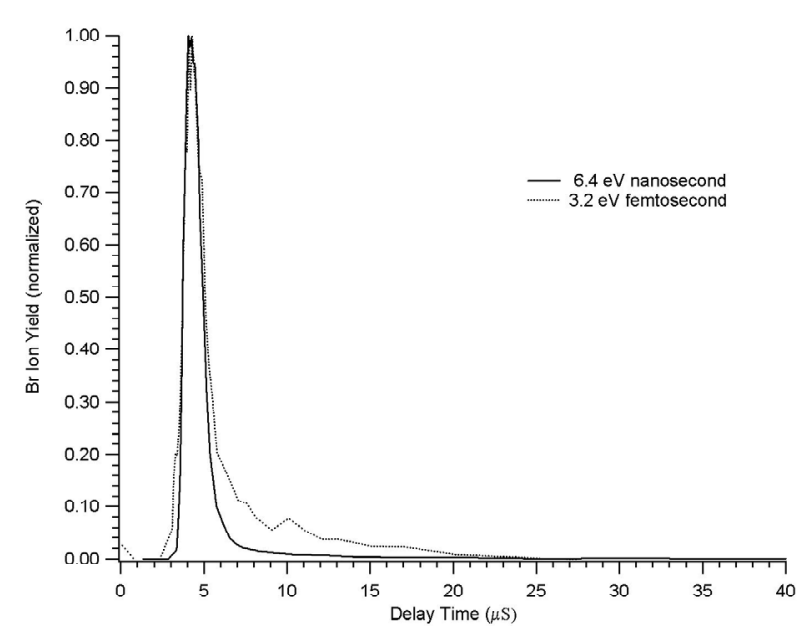

Figure 3.3. $\mathrm{Br}$ atom yield as a function of delay between the excitation and probe lasers following excitation using a $6.4 \mathrm{eV}$ nanosecond pulse; fluence $=0.003 \mathrm{~mJ} / \mathrm{cm}^{2}$ (solid line) and a $3.2 \mathrm{eV}$ femtosecond pulse; fluence $=0.6 \mathrm{~mJ} / \mathrm{cm}^{2}$ (dotted line). Very weak $\mathrm{Br}^{*}$ emission (not shown) is observed under these conditions.

hyper-thermal emission, as expected for $6.4 \mathrm{eV}$ excitation (Hess et al. in press), the $3.2 \mathrm{eV}$ irradiation gives rise also to a broad tail, including both thermal and intermediate velocity components. This tail is much weaker in the case of the single-photon $6.4 \mathrm{eV}$ excitation. In both cases it can be caused by $6.4 \mathrm{eV}$ excitation of bulk and near-surface excitons in the Urbach tail (Tomiki et al. in press). H centers created by decomposition of these excitons can reach the surface and give rise to the thermal component (Pachin et al. 1993). The number of these centers produced in a powerful $3.2 \mathrm{eV}$ fs pulse is larger than that in a relatively weak $6.4 \mathrm{eV}$ ns pulse. Presence of the intermediate velocity component, however, suggests that perhaps more complex processes are taking place. The yield of these processes is small due to the low effectiveness of bulk exciton absorption at $6.4 \mathrm{eV}$. Therefore excitation with higher energy photons could shed more light on these processes.

Figure 3.4 displays the velocity profiles of $\mathrm{Br}$ and $\mathrm{Br}^{*}$ following $3.5 \mathrm{eV}$ and $7.0 \mathrm{eV}$ nano-second excitation. Although $3.5 \mathrm{eV}$ excitation is far below the first UV absorption band, at high fluence $\left(25 \mathrm{~mJ} / \mathrm{cm}^{2}\right)$ abundant bromine is produced (Beck et al. 2001). If the $\mathrm{Br}$ yield resulted solely from two-photon excitation at $3.5 \mathrm{eV}$, one would expect results similar to the onephoton excitation at $7.0 \mathrm{eV}$. However, unlike $6.4 \mathrm{eV}$ vs. $3.2 \mathrm{eV}$ fs excitation, there are dramatic differences 


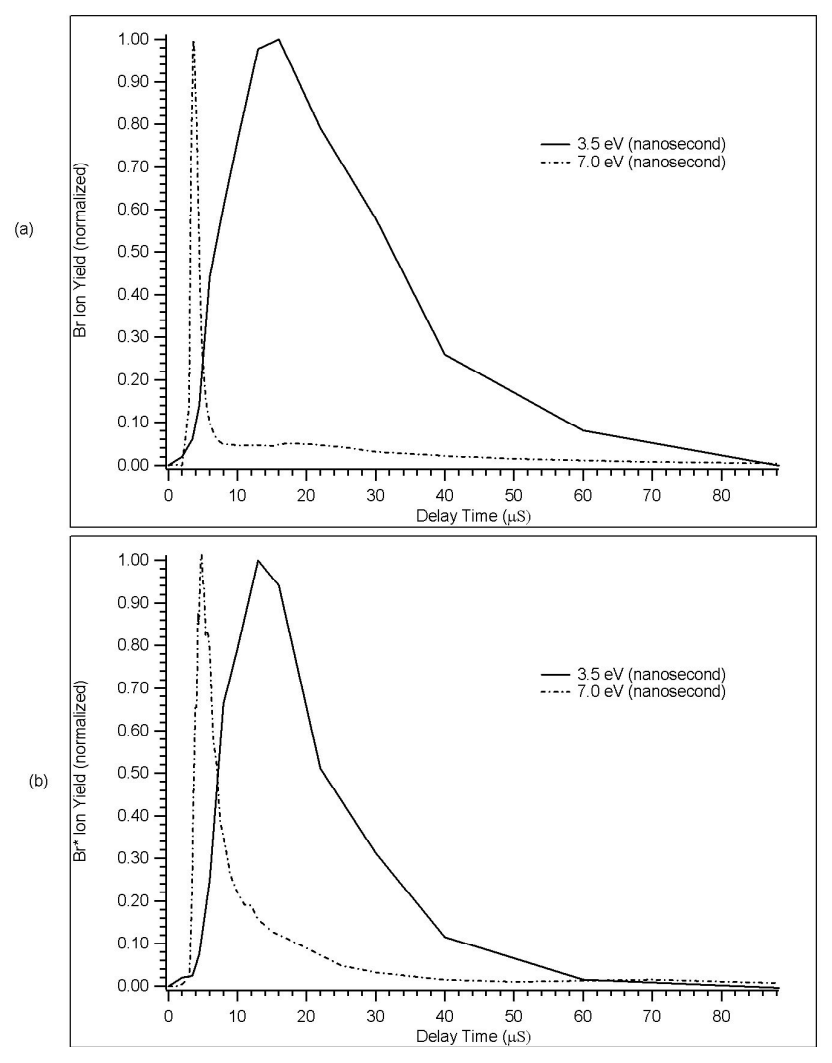

Figure 3.4. Bromine atom yield as a function of delay between the excitation and probe lasers for $\mathrm{Br}$ (a) and $\mathrm{Br}^{*}$ (b) for both $3.5 \mathrm{eV}$; fluence $=25 \mathrm{~mJ} / \mathrm{cm}^{2}$ (solid lines) and $7.0 \mathrm{eV}$; fluence $=0.06 \mathrm{~mJ} / \mathrm{cm}^{2}$ (dashed lines) nanosecond excitation. Very minor $\mathrm{Br}^{*}$ emission is observed under $7.0 \mathrm{eV}$ excitation whereas the total $\mathrm{Br}$ and $\mathrm{Br}^{*}$ yields are similar for $3.5 \mathrm{eV}$ excitation.

between $3.5 \mathrm{eV}$ and $7.0 \mathrm{eV}$ ns velocity profiles. The power dependence of the $3.5 \mathrm{eV}$ excitation laser is $\mathrm{P}^{2.7 \pm 0.2}$, greater than the quadratic dependence expected for a pure two-photon process. A fast, "hyper-thermal" component (the extremely narrow peak centered near $4 \mu \mathrm{s}$ ) dominates the weak thermal "tail" following $7.0 \mathrm{eV}$ excitation while at $3.5 \mathrm{eV}$, the signal is dominated by the slower distribution.

Clearly, excitation with $3.5 \mathrm{eV}$ ns pulses does not produce the expected result if only two-photon excitation is taken into account. This apparent discrepancy may be explained by formation and excitation and has a broad absorption centered at $3.2 \mathrm{eV}$ (Shluger and Tanimura 2000; Shluger et al. 1995). Formation and secondary excitation of these transient species by $3.5 \mathrm{eV}$ photons are therefore possible within a single nanosecond pulse and could be responsible for the dominant "thermal-like" emission component evident in Figure 3.4. This would also explain the highly nonlinear power dependence at $3.5 \mathrm{eV}$. Because formation of transient centers is likely a two-photon process and secondary excitation is a single photon process, the bromine emission power dependence is greater than $\mathrm{P}^{2}$ but less than $\mathrm{P}^{3}$.

The $\mathrm{Br}$ and $\mathrm{Br}^{*}$ velocity distributions in Figure 3.4a and $b$ have similar but non-identical velocity profiles. The relative yield of $\mathrm{Br}^{*}$ is nearly equal to $\mathrm{Br}$ under $3.5 \mathrm{eV}$ excitation whereas the Br yield greatly dominates the Br* yield for 6.4 (Hess et al. In press), $7.0 \mathrm{eV}$ nanosecond and $3.2 \mathrm{eV}$ femtosecond excitation. This is consistent with the mechanism involving secondary excitation of $\mathrm{H}$ centers. If we assume that the electronic excitation of $\mathrm{H}$ centers stimulates diffusion of $\mathrm{Br}$ and $\mathrm{Br}^{*}$, this mechanism could also explain the high $\mathrm{Br}^{*}$ yield at $3.5 \mathrm{eV}$. Although the $3.2 \mathrm{eV}$ photon energy of the femtosecond pulse is strongly resonant with both $\mathrm{V}_{\mathrm{K}}$ and $\mathrm{H}$ center optical absorption, significant "thermal-like" emission is not observed at this excitation. This is primary because the $\mathrm{V}_{\mathrm{K}}$ and $\mathrm{H}$ center formation time is significantly longer than the $\sim 150$ fs pulse duration and only a very small number of $\mathrm{H}$ centers are expected to be formed during the pulse (Shluger and Tanimura 2000).

A number of conclusions can been drawn. First, twophoton excitation creates transient centers in $\mathrm{KBr}$. Second, once transient centers are formed secondary excitation is relatively facile for nanosecond pulsed excitation and leads to strong $\mathrm{Br}$ and $\mathrm{Br} *$ emission. Third, the similarity of $\mathrm{Br}$ and $\mathrm{Br}$ * velocity profiles and yields under $3.5 \mathrm{eV}$ excitation suggests a common source for both photoproducts. The interstitial $\mathrm{H}$ center is somewhat more likely to undergo photo decomposition to desorb $\mathrm{Br}$ and $\mathrm{Br}^{*}$.

\section{Acknowledgements}

The Divisions of Chemical Sciences of the Office of Basic Energy Sciences supported the authors. Battelle operates Pacific Northwest National Laboratory for the U.S. Department of Energy under contract number DE-AC06-76RLO1830. ALS would like to thank the Leverhulme Trust for financial support and $\mathrm{K}$. Tanimura for very useful discussions. 


\section{References}

Alexandrov, A., M. Piacentini, N. Zema, A. C. Felici, and T. M. Orlando, Phys. Rev. Lett. 86, 536 (2001).

Beck, K. M., A. G. Joly, and W. P. Hess, Phys. Rev. B 63, 125423 (2001).

Hess, W. P., A. G. Joly, D. P. Gerrity, K. M. Beck, P. V. Sushko, and A. L. Shluger, J. Chem. Phys. (in press).

Kolodziej, J., P. Piatkowski, and M. Szymonski, Surf. Sci., 390, 152 (1997).

Puchin, V. E., A. L. Shluger, and N. Itoh, Phys. Rev. B 47, 10760 (1993).

Shluger, A. L. and K. Tanimura, Phys. Rev. B 61, 5392 (2000).

Shluger, A. L., V. E. Puchin, T. Suzuki, K. Tanimura, and N. Itoh, Phys. Rev. B 52, 4017 (1995).

Such, B., P. Czuba, P. Piatkowski, and M. Szymonski, Surf. Sci. 451, 203 (2000).

Tomiki, T., T. Miyata, and H. Tsukamoto, Z. Naturforsch 29A, 145 (1974).

Zema, N., M. Piacentini, P. Czuba, J. Kolodziej, P. Piatkowski, Z. Postawa, and M. Szymonski, Phys Rev. B. 5, 5448 (1997).

\section{Selective Laser Desorption of Ionic Surfaces: Resonant Surface Excitation of $\mathrm{KBr}$}

W. P. Hess, ${ }^{(a)}$ A. G. Joly, ${ }^{(a)}$

D. P. Gerrity, ${ }^{(b)}$ K. M. Beck, ${ }^{(a)}$

P. V. Sushko, ${ }^{(c)}$ and A. L. Shluger ${ }^{(c)}$

Supported by DOE Office of Basic Energy

Sciences, Chemical Sciences Division.

(a) Pacific Northwest National Laboratory.

(b) Department of Chemistry, Reed College, Portland, OR.

(c) Department of Physics and Astronomy, University College London.

Electronic excitation of solids creates bulk and surface defects, induces surface decomposition and particle emission, and leads to desorption of adsorbed species, electron emission, and other processes. Many of these processes, such as desorption induced by electronic transitions (DIET) and laser ablation, have many technological applications and have been studied in great detail. However, surface desorption of materials by electronic excitation is difficult to control. One of the main reasons is that available irradiation sources, such as ion and electron beams, X-rays, and lasers induce a variety of different processes inside a solid. Since the mechanisms of relaxation of electronically excited states in most cases are poorly understood, selectively inducing a particular surface desorption mechanism has been difficult.

In this report, we combine theoretical calculations with sensitive laser desorption experiments on cleaved $\mathrm{KBr}$ single crystals. We have achieved selective desorption of hyper-thermal $\mathrm{Br}$ atoms by using laser excitation in the Urbach tail of the bulk exciton absorption of $\mathrm{KBr}$. In addition, we produce an extremely large excess of the $\operatorname{Br}\left({ }^{2} \mathrm{P}_{3 / 2}\right)$ ground state over the spin orbit excited $\operatorname{Br}\left({ }^{2} \mathrm{P}_{1 / 2}\right)$ state $\left(\mathrm{Br}^{*}\right)$. We calculate transition energies for specific surface structures such as terraces, steps, and kinks and argue that surface specific emission of neutral halogen atoms is induced by resonant excitation of excitons on surface terraces at $6.4 \mathrm{eV}$. We note that the principles of resonant surface excitation are general and likely extend not only to other alkali halides but also to technologically important materials, such as oxides. Using this approach it may be possible to tailor surface excitation to control solid-state reaction in a variety of useful materials (Hess et al. 2001).

The ultra-high vacuum apparatus (UHV) and data treatment used in these experiments are described in detail in the literature (Beck et al. 1997). We irradiate the $\mathrm{KBr}$ surface at room temperature using laser pulses of $3 \mathrm{~ns}$ duration and fluence ranging between 1 and $10 \mu \mathrm{J} / \mathrm{cm}^{2}$, depending on photon energy. The weak nanosecond excitation laser pulses result in emission of bromine and potassium atoms. The probe laser pulse is focused by a $200 \mathrm{~mm}$ quartz lens to intersect the desorbed atoms $3.8 \mathrm{~mm}$ above, and oriented parallel to, the sample surface. Tunable $1.0 \mathrm{~mJ}$ pulses from a $20 \mathrm{~Hz}, 5 \mathrm{~ns} \mathrm{Nd}$ :YAG pumped frequency-doubled dye laser are used to ionize ground $\operatorname{Br}\left({ }^{2} \mathrm{P}_{3 / 2}\right)$ and excited state $\operatorname{Br}\left({ }^{2} \mathrm{P}_{1 / 2}\right)$ atoms in a $(2+1)$ resonance-enhanced multiphoton ionization (REMPI) scheme. Atomic masses are determined by a time-offlight (TOF) mass spectrometer using chevron microchannel plates to amplify the ion signal. Each data point represents an average of the integrated mass selected ion signal from 200 laser pulses.

To study the electronic transitions of the $\mathrm{KBr}$ surface and the mechanisms of emission of $\mathrm{Br}$ atoms, we use an embedded cluster method described in detail 
elsewhere (Sushko et al. 2000). Briefly, we consider a cluster of ions treated quantum-mechanically (quantum or QM cluster) embedded in a large finite array of polarizable ions. The latter in its turn is divided into two regions. Several hundred ions closest to the quantum cluster are treated in the shell model (Dick and Overhauser, 1958) and make up the central region. In the shell model, an ion is represented by a point core and shell connected by a spring to simulate its dipole polarizability. The positions of the cores and shells of each of the ions in the central region are optimized in response to the changes in the charge density distribution within the quantum cluster to minimize the total energy of the whole system. The rest of point ions outside the central region form the outer region of non-polarizable ions fixed in the perfect lattice sites.

Recent experimental results have suggested that the surface exciton on alkali halides may be selectively excited (Beck et al. 2001). Previous work (Eby et al. 1959) and more recent synchrotron radiation experiments demonstrated that the optical absorption of $\mathrm{KBr}$ at room temperature starts at about $6 \mathrm{eV}$ with the first exciton band center located near 6.6 eV (Eby et al. 1959). Therefore, $6.4 \mathrm{eV}$ photons can directly excite excitons in the bulk by absorption in the Urbach tail and possibly at the surface if there is surface exciton absorption. On the other hand, $7.9 \mathrm{eV}$ photons can excite electron-hole pairs in the bulk and can ionize surface sites producing surface holes. Selective surface excitation is possible if the transition energy is shifted from that of the bulk.

Figure 3.5 shows the normalized raw velocity profiles of $\mathrm{Br}$ following 6.4 and $7.9 \mathrm{eV}$ excitation. A narrow hyper-thermal component peaking at $3.8 \mu \mathrm{s}$ is observed in the arrival time data at $6.4 \mathrm{eV}$. The velocity distribution generated using $7.9 \mathrm{eV}$ excitation is markedly different. There is a clear near-thermal component peaking near $16 \mu$ s. The $7.9 \mathrm{eV}$ laser fluence is approximately ten times greater than that used at $6.4 \mathrm{eV}$ to achieve the same detectable levels of $\mathrm{Br}$ emission (Beck et al. 2001). The emission yield shows a linear power dependence of $\mathrm{P}^{0.9 \pm .2}$ indicating that the $6.4 \mathrm{eV}$ photons are resonant with a surface excitation despite being below the first bulk exciton band at $\sim 6.6 \mathrm{eV}$ (Zema et al. 1997).

To estimate a possible shift of the surface excitation energy with respect to the bulk we have calculated the

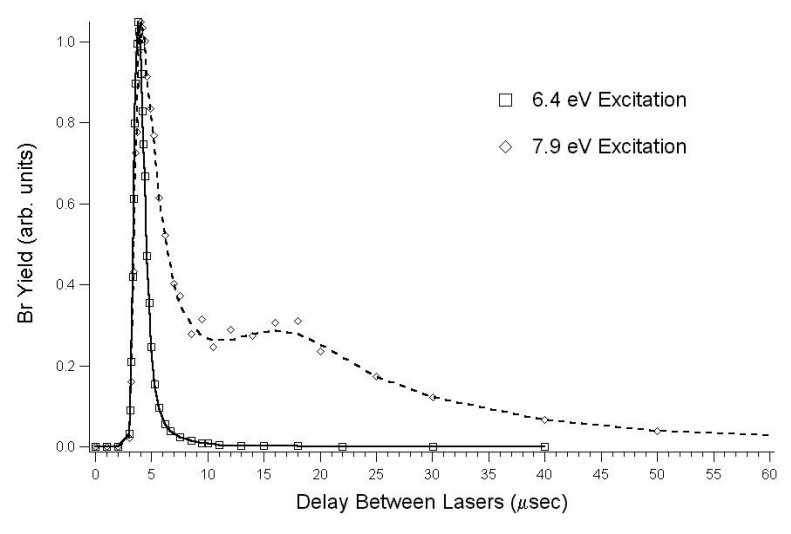

Figure 3.5. Velocity profiles of $\mathrm{Br}$ emission following 6.4 and $7.9 \mathrm{eV}$ excitation. The $6.4 \mathrm{eV}$ distribution (squares) display only the hyperthermal component while the $7.9 \mathrm{eV}$ distribution (dots) display both hyperthermal and near-thermal components. Both curves have been normalized.

TD-DFT excitation energies for the three QM clusters $\mathrm{K}_{6} \mathrm{Br}_{6}, \mathrm{~K}_{9} \mathrm{Br}_{9}$, and $\mathrm{K}_{18} \mathrm{Br}_{18}$. Each of these clusters is three-layers deep where the top layer is in the surface plane. The results demonstrate the hierarchy of excitation energies near the surface, which is mainly due to the difference in the electrostatic potential in different surface layers. The excitation in the surface layer has the lowest energy and is split by about $0.2 \mathrm{eV}$ from higher energy excitations with the charge transfer from the sub-surface layers to the surface.

To model the surface exciton relaxation after excitation we consider the lowest triplet excited state, which can be calculated self-consistently, as in our previous calculations (Shluger and Tanimura 2000; Shluger et al. 1993). This lowest surface excited state is initially strongly delocalized on the perfect surface, as is demonstrated by the spin density plot in Figure 3.6. The lowest energy relaxation path corresponds to a displacement of one of the surface $\mathrm{Br}$ ions out of the surface plane. This leads to an additional localization of the spin density around that ion and to a force displacing the ion outward and perpendicular to the surface plane.

Our results demonstrate a very different character of desorption of $\mathrm{Br}$ atoms under excitation at $6.4 \mathrm{eV}$ and $7.9 \mathrm{eV}$ : the $6.4 \mathrm{eV}$ excitation generates nearly pure hyper-thermal $\mathrm{Br}$ emission, whereas the $7.9 \mathrm{eV}$ excitation generates both near-thermal and hyper-thermal emission. We conclude that the lack of a near-thermal component following $6.4 \mathrm{eV}$ excitation indicates that 


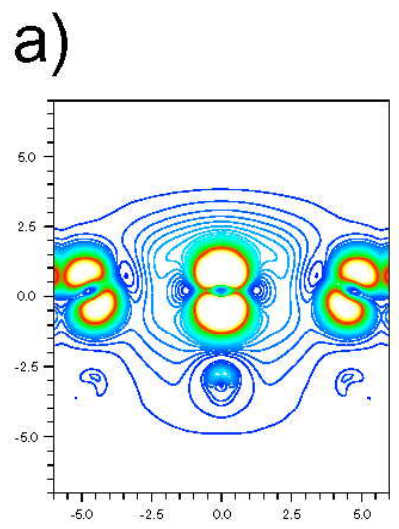

b)

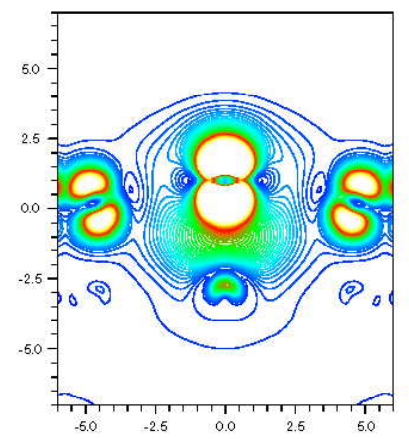

c)

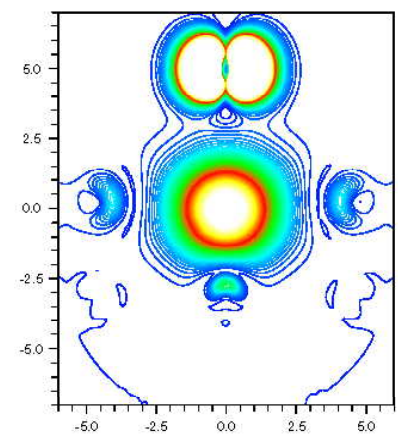

Figure 3.6. Spin density for the triplet surface exciton: (a) unrelaxed exciton, (b) initial stages of the relaxation ( $\mathrm{Br}$ atoms is leaving the surface), and (c) $\mathrm{Br}$ atom is about $5 \AA$ from the surface.

bulk excitation results in negligible desorption compared to that generated by surface excitation. The effective first-order process suggests that $6.4 \mathrm{eV}$ photons can directly excite surface excitons. Our calculations demonstrate that the surface absorption threshold is shifted to lower energies from the bulk value, due to the lower coordination of terrace, step, and corner sites, confirming the possibility of selective surface excitation. The predicted surface excitation energy of $6.2 \mathrm{eV}$ is close to the experimental photon energy of $6.4 \mathrm{eV}$. We are currently working on measuring the hyper-thermal desorption yield as a function of photon energy in the region of 6.0-7.1 eV. Our high sensitivity may allow us to establish REMPI as a method for selective studies of surface states.

\section{References}

Beck, K. M., A. G. Joly, and W. P. Hess, Phys.

Rev. B. 63, 125423 (2001).

Beck, K. M., D. P. Taylor, and W. P. Hess, Phys. Rev. B. 55, 13253 (1997).

Dick, B. G. and A. W. Overhauser, Phys. Rev. 112, 90 (1958).

Eby, J. E., K. J. Teegarden, and D. B. Dutton, Phys. Rev. 116, 1099 (1959).

Hess, W. P., A. G. Joly, D. P. Gerrity, K. M. Beck, P. V. Sushko, and A. L. Shluger, J. Chem. Phys. 115, 9463 (2001).

Shluger, A. L. and K. Tanimura, Phys Rev. B. 61, 5392 (2000).

Shluger, A. L., A. H. Harker, V. E. Puchin, N. Itoh, and C. R. A. Catlow, Modelling Simul. Mater. Sci. Eng. 1, 673 (1993).
Sushko, P. V., A. L. Shluger and C. R. A. Catlow, Surface Sci. 450, 153 (2000).

Zema, N., M. Piacentini, P. Czuba, J. Kolodziej, P. Piatkowski, Z. Postawa, and M. Szymonski, Phys Rev. B 55, 5448 (1997).

\section{Femtosecond Time-Resolved Photo-Stimulated Desorption of Positive Ions from Ionic Crystals}

A. G. Joly, ${ }^{(a)}$ W. P. Hess, ${ }^{(a)}$

K. M. Beck, ${ }^{(a)}$ and J.T. Dickinson ${ }^{(b)}$

Supported by DOE office of Basic Energy Sciences, Chemical Sciences Division

(a) Pacific Northwest National Laboratory.

(b) Department of Physics, Washington State University.

Laser ablation and desorption techniques are used extensively for a diverse range of disciplines including the production of new materials and extrinsic or in situ chemical analysis (Russo et al. 1997, Horwitz et al. 1999). Unfortunately, no detailed mechanistic understanding of the processes leading to ion emission has emerged due to the inherent complexity of the laser excitation and subsequent particle emission processes. To date, most laser desorption/ablation processes have used nanosecond pulses due to their reliability even though many of the primary processes leading to particle emission occur on a sub-nanosecond timescale. For this reason, experiments using femtosecond laser 
pulses may provide unique insight and serve to clarify the steps involved in the desorption process. For example, the high peak intensities of femtosecond pulses allows multiphoton absorption paths to higher excited levels than is possible using nanosecond pulses near the ion emission threshold. Thus, near threshold, the ion desorption mechanism is different for femtosecond and nanosecond laser excitation.

Femtosecond UV pulses are produced in an amplified titanium-sapphire laser system operating at a fundamental wavelength of $795 \mathrm{~nm}$ at a repetition rate of $20 \mathrm{~Hz}$. The amplified output is frequency doubled and tripled in KDP crystals producing 150 fs pulses with $1.5 \mathrm{~mJ} /$ pulse at $265 \mathrm{~nm}$. The ultrafast pulse is split into a pair of approximately equivalent pulses. One pulse is directed through a variable distance optical delay line and the pulse pair is recombined, at small angle, on the sample surface. The sample is mounted on a variable temperature manipulator inside an ultra-high vacuum chamber with a base pressure of $4 \times 10^{-10}$ Torr and equipped with a Wiley-McLaren time-of-flight mass spectrometer. The pulse energy is adjusted such that each pulse is near, but below, threshold for ion emission/detection. A single mass/charge peak may be integrated and averaged versus delay between laser pulses.

Figure 3.7 displays the $\mathrm{Mg}^{+}$ion signal plotted as a function of delay between the fs laser pulses following excitation of single crystal $\mathrm{MgO}$. The figure inset displays an expanded short-time region. Clearly there are at least three distinct time regions: (1) a narrow coherence peak centered at zero delay with an approximately Gaussian profile; (2) a fast, picosecond growth and (3) a slow $\sim 25$ picosecond decay. In addition, a longer $>60$ picosecond decay (not shown) has been reported previously (Hess et al. 1999). We have measured the ion yield versus laser power dependence following femtosecond irradiation and found it to be between $\mathrm{P}^{4}$ and $\mathrm{P}^{5}$. A similar nonlinear power dependence was reported for $\mathrm{MgO}$ under nanosecond pulse excitation and a "multiple" photon model was proposed to explain ion desorption at much lower laser intensities (Ermer et al. 1996).

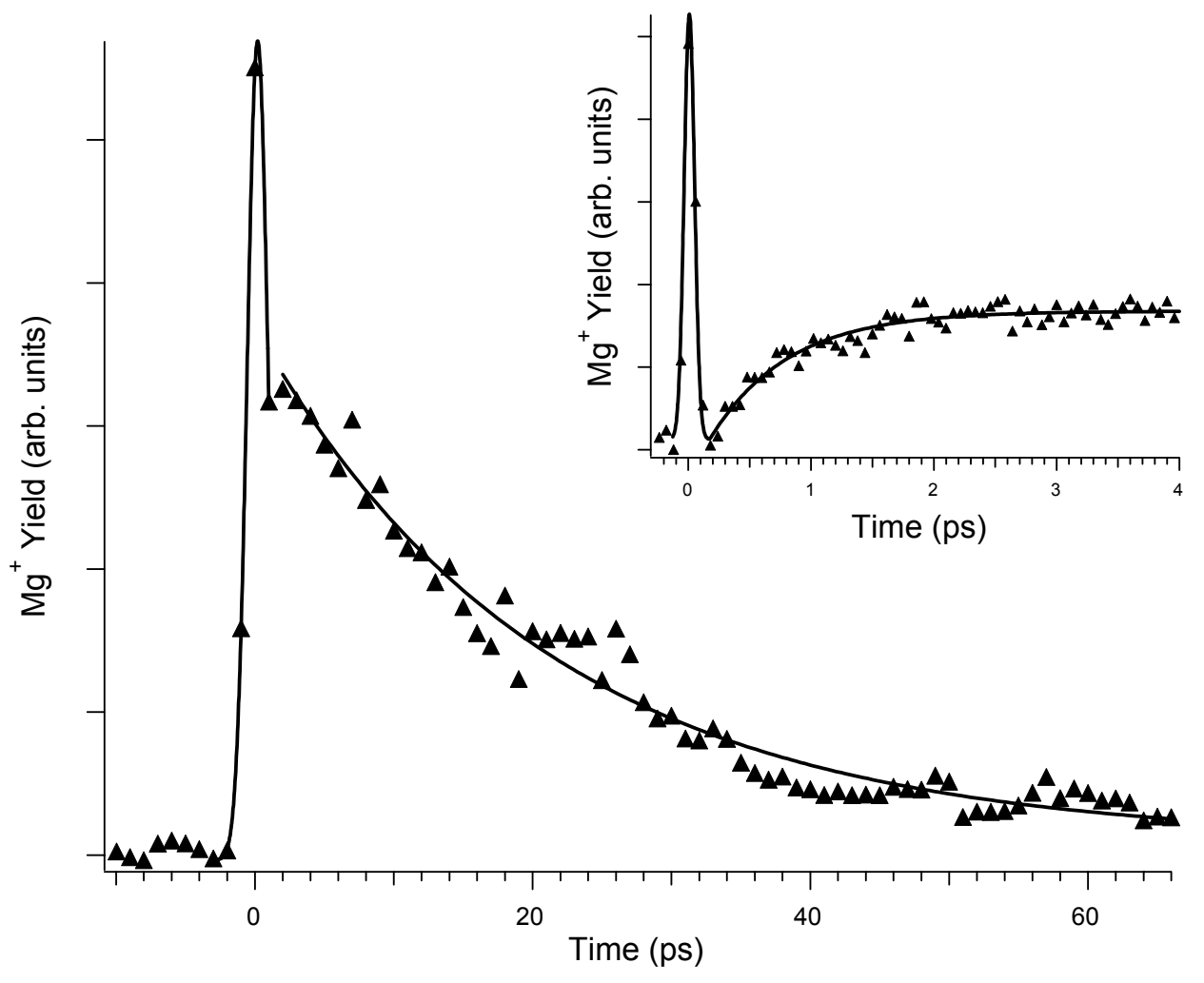

Figure 3.7. The desorption yield of $\mathrm{Mg}^{+}$from single crystal $\mathrm{MgO}$ as a function of delay between pump and probe pulses showing three distinct time regimes. The inset shows an expanded view of the short-time region. 
There are a number of processes which may lead to desorption of positive ions following either femtosecond or nanosecond excitation. The highly nonlinear power dependence argues strongly that multiphoton absorption is the predominant excitation mechanism resulting in positive ion desorption following ultrashort single-pulse excitation. Such multiphoton excitation would be expected to produce photoelectron emission, hot conduction band electrons, and hot holes in addition to exciting electron traps such as $\mathrm{F}$ and $\mathrm{F}^{+}$centers.

The fast $\sim 1$ picosecond growth feature displayed in Figure 3.7 is significantly longer than the pulse duration and is independent of the polarization combinations of the pump and probe pulses. The growth feature represents the evolution of the initially excited state or precursor state through either relaxation or actual reaction to a transient state that may further interact with either photons from the probe pulse or new species directly created by the probe pulse. It is the combined interactions that result in $\mathrm{Mg}^{+}$emission in delayed pulse pair excitation. The transient intermediate state then decays on the longer (20 picosecond and greater) timescale. The transient state lifetime is commensurate with the 50 picosecond free carrier lifetime measured using a femtosecond interferometric technique (Petite et al. 1997).

That the intermediate state survives for several tens of picoseconds, over which time it may interact with the probe pulse, and provides an attractive explanation for the mechanism of nanosecond desorption in the lowfluence regime. Since the formation of this state takes roughly a picosecond and it survives for several tens of picoseconds, a single nanosecond pulse could both create the initial precursor and provide subsequent excitation necessary for ion emission. In contrast, femtosecond pulses lead to desorption primarily through a coherent multiphoton absorption process as evidenced by the coherence peak. In essence, although the material excitation (precursor state) exists to produce $\mathrm{Mg}^{+}$ions through multiple single photon events, the pulse duration is too short relative to the intermediate state's formation time to induce such a mechanism.

Potassium bromide $(\mathrm{KBr})$ is also a widely studied insulating crystal. Unlike $\mathrm{MgO}$, however, the $\mathrm{KBr}$ crystal is formed by singly instead of doubly charged ions. The differences in electronic structure allow the formation of self-trapped holes in bulk $\mathrm{KBr}$, in contrast to $\mathrm{MgO}$ (Shluger et al. 1991). Figure 3.8 displays the results from fs pump-probe experiments performed on single crystal $\mathrm{KBr}$. Figure 3.8 shows a large sharp feature at zero time, and then a slower $\sim 20$ picosecond decay in the $\mathrm{K}^{+}$signal. In contrast to $\mathrm{MgO}$, there is no observable prominent rise following the coherence peak at zero delay time within our signal/noise. Once again, $\mathrm{KBr}$ allows one to understand the mechanistic differences between femtosecond and nanosecond desorption. The short-time, multiphoton feature is presumably responsible for femtosecond desorption while the longer 20 picosecond tail allows subsequent single-photon absorption steps for nanosecond pulses. Further experiments are underway to elucidate the fundamental processes involved in desorption of positive ions from $\mathrm{KBr}$ and other wide bandgap ionic crystals.

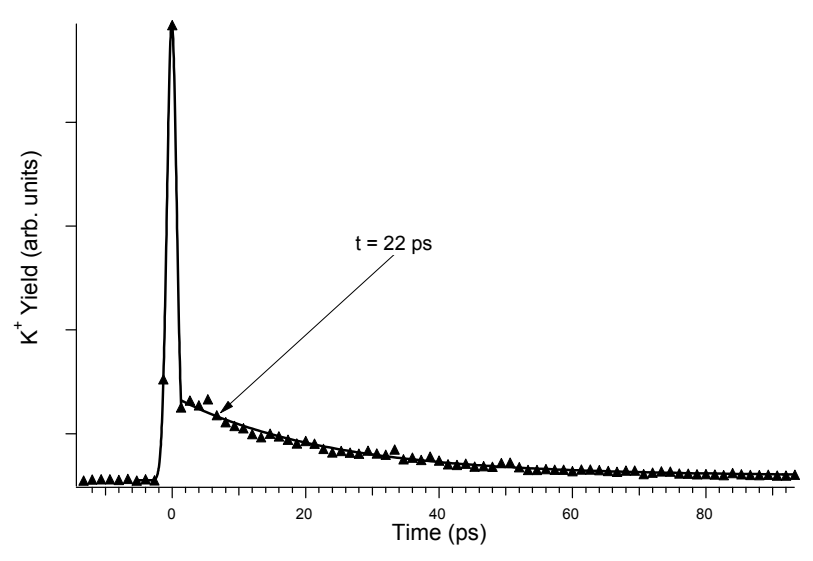

Figure 3.8. The desorption yield of $\mathrm{K}^{+}$from single crystal $\mathrm{KBr}$ as a function of delay between pump and probe pulses. In contrast to $\mathrm{MgO}$, within our signal/noise, there is no observable short-time rise after the coherence feature at zero time.

\section{References}

Ermer, D. R., J.-J. Shin, S. C. Langford, K. W. Hipps, and J. T. Dickinson, J. Appl. Phys 80, 6452 (1996).

Hess, W. P., A. G. Joly, K. M. Beck, R. M. Williams, and J. T. Dickinson, Applied Phys. A 69, S389 (1999).

Horwitz, J. S., H.-U. Krebs, K. Murakami, and M. Stuke (Eds), Proceedings of the Fifth International Conference on Laser Ablation (COLA 99), Springer-Verlag, Heidelberg, 1999.

Petite, G., P. Daguzan, S. Guizard, and P. Martin, Applied. Surf. Sce. 109/110, 36 (1997). 
Russo, R. E., D. B. Geohegan, J. R. F. Haglund, and K. Murakami (Eds), Proceedings of the Fourth International Conference on Laser Ablation (COLA 97), Elsevier, Amsterdam, Monterey, 1997. Shluger, A. L., R. W. Grimes, C. R. A. Catlow, and N. Itoh, J. Phys. Condens. Matter 3, 8027 (1991).

\section{Up-Conversion Luminescence of $\mathrm{Mn}^{2+}$ in $\mathrm{ZnS}: \mathrm{Mn}^{2+}$ Nanoparticles}

\author{
W. Chen, ${ }^{(a)}$ A. G. Joly, ${ }^{(b)}$ and \\ J. Z. Zhang ${ }^{(c)}$ \\ (a) Nomadics, Inc. \\ (b) Pacific Northwest National Laboratory. \\ (c) Department of Chemistry, University of \\ California, Santa Cruz.
}

Due to potential applications in lasers, laser cooling, optical communications, storage, displays, and imaging techniques, up-conversion luminescence has been extensively investigated (see Lenth and MacFarlane 1992). Up-conversion, often referred to as anti-Stokes luminescence, is a process where the luminescence emitted from the material is at higher energy than the excitation source and is well documented in rare earth compounds and a wide variety of nanoscale materials. Auger recombination, energy transfer, and two-photon absorption processes have been proposed to explain these observations in semiconductor quantum well structures and nanoparticles.

Optical properties of semiconductor nanoparticles have been extensively studied in recent years (Halwa 2000). Due to quantum confinement, a large surfaceto-volume ratio, and the geometrical confinement of phonons, semiconductor nanoparticles behave differently than bulk materials and have some novel properties and effects. $\mathrm{Mn}^{2+}$ doped materials represent a class of phosphors that have already been used for many applications. Transition metal ion doped semiconductor nanoparticles may display unique and interesting properties worth studying for both basic research and application purposes.

The average sizes of the particles used in this study as estimated from high-resolution transmission electron microscope and X-ray diffraction, are approximately $1,3,3.5,4.5$, and $10 \mathrm{~nm}$. The $1 \mathrm{~nm}$ sized $\mathrm{ZnS}: \mathrm{Mn}^{2+}$ nanoparticles are encapsulated in an ultrastable zeolite-Y (USY), while the $10 \mathrm{~nm}$ nanoparticles are naked particles without any capping. The 3, 3.5, and $4.5 \mathrm{~nm}$ particles are capped with methacrylic acid/citric acid.

The photoluminescence and up-conversion emission spectra and lifetime data were collected using a nanosecond optical parametric oscillator/amplifier (OPO) at a $10 \mathrm{~Hz}$ repetition rate and tunable between $440 \mathrm{~nm}$ and $1800 \mathrm{~nm}$. The output of the OPO system was either frequency doubled in a KDP crystal for observation of the photoluminescence emission or used directly for the up-conversion measurements. The output was directed onto the particles. Emission was collected at right angles to the excitation and focused into a $1 / 8$ meter monochromator with either a gated intensified CCD detector (for emission spectra) or a standard photomultiplier tube (for lifetime measurement).

Figure 3.9 shows the photoluminescence (excited at $300 \mathrm{~nm}$ ) and up-conversion (excited at $767 \mathrm{~nm}$ ) emission spectra of the $\mathrm{ZnS}: \mathrm{Mn}^{2+}$ nanoparticles. For comparison, the up-conversion emission spectrum of bulk $\mathrm{ZnS}: \mathrm{Mn}^{2+}$ is also shown. The broad emission is from the ${ }^{4} \mathrm{~T}_{1} \rightarrow{ }^{6} \mathrm{~A}_{1}$ transition of $\mathrm{Mn}^{2+}$. To see the difference between the photoluminescence and the up-conversion emissions, the two spectra are normalized in each sample. Figure 3.9 clearly shows that for the bulk and the $1 \mathrm{~nm}$ sized $\mathrm{ZnS}: \mathrm{Mn}^{2+}$ particles encapsulated in USY, the up-conversion emission spectra are almost identical with the photoluminescence emission spectra. However, for the 3, 3.5, 4.5, and $10 \mathrm{~nm}$ nanoparticles, the up-conversion emission maxima are redshifted by approximately $7.5,9,6$, and $4 \mathrm{~nm}$ from their photoluminescence emission maxima.

Figure 3.10 shows the lifetime decay curves of the photoluminescence excited at $300 \mathrm{~nm}$ and upconversion luminescence excited at $767 \mathrm{~nm}$. It is interesting to note that the up-conversion decay lifetimes are shorter than the corresponding photolumi nescence decay lifetimes. Excitation power dependences show that the photoluminescence is due to a single photon excitation while the up-conversion luminescence intensity displays a quadratic dependence on excitation power, indicating a second order process. 


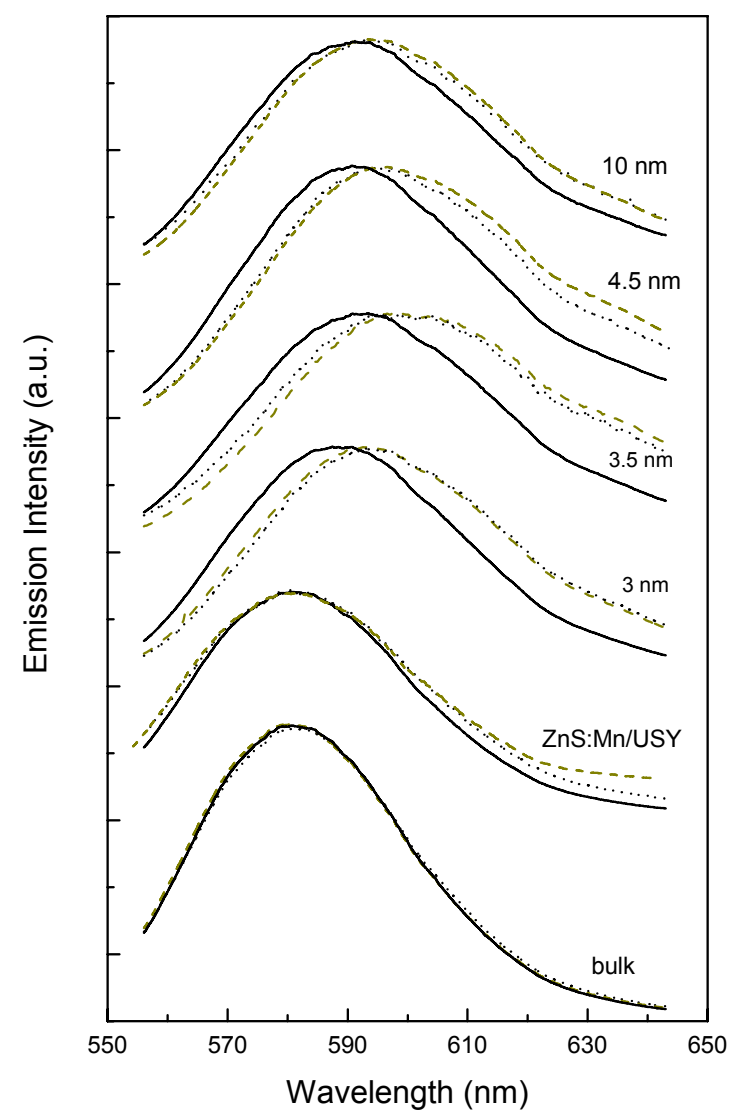

Figure 3.9. The photoluminescence spectra after excitation at $300 \mathrm{~nm}$ (solid lines), at $383.5 \mathrm{~nm}$ (dashed lines), and the up-conversion luminescence spectra resulting from $767 \mathrm{~nm}$ excitation (dotted lines) of bulk $\mathrm{ZnS}: \mathrm{Mn}^{2+}$ and different sized nanoparticles.

Both Auger recombination and two-photon absorption can result in quadratic dependence of the luminescence intensity on the excitation power (Cho et al. 1997; Pollnau et al. 2000). Emission spectra obtained with single photon energy $(383.5 \mathrm{~nm})$ which is the sum energy of two photons at $767 \mathrm{~nm}$ are nearly identical with the up-conversion spectra as displayed in Figure 3.9. In addition, the luminescence lifetimes obtained with $383.5 \mathrm{~nm}$ excitation are nearly identical with the up-conversion luminescence lifetimes. The emission spectra and lifetimes obtained at $383.5 \mathrm{~nm}$ excitation should be the same as that at $767 \mathrm{~nm}$ excitation if the up-conversion luminescence is due to two-photon excitation, because the excitation energies are actually the same in this case. If the upconversion luminescence is due to Auger processes, the spectra and lifetimes excited at $383.5 \mathrm{~nm}$ are not expected to be the same as those at $767 \mathrm{~nm}$ excitation, because the two energies are not the same. This

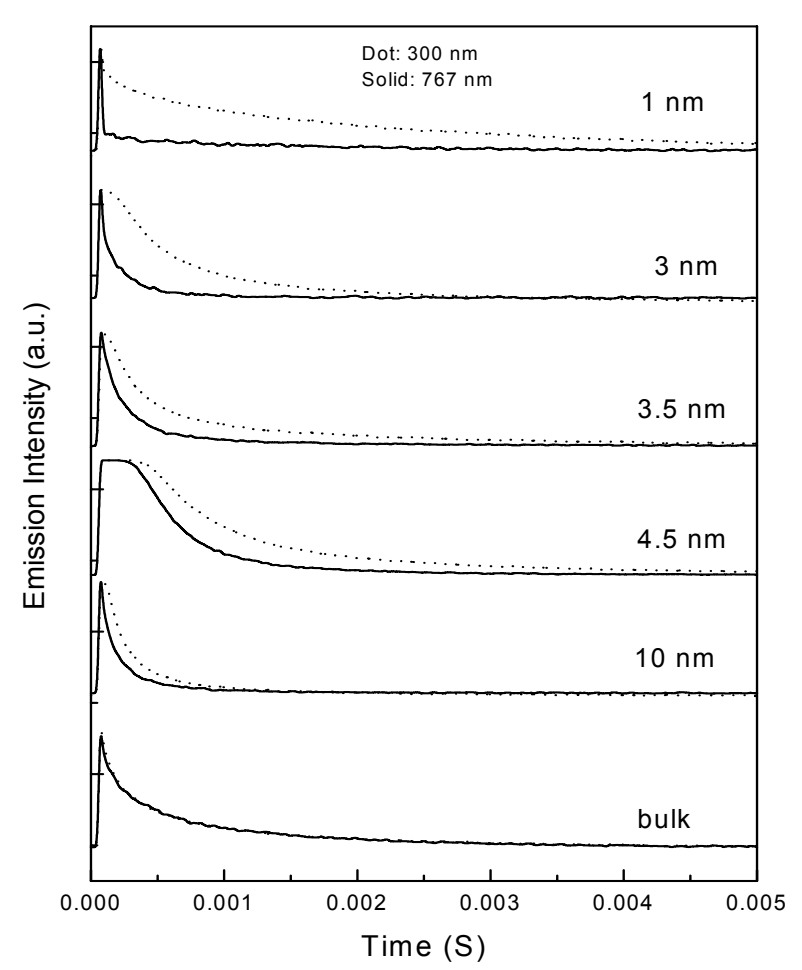

Figure 3.10. Lifetime decays of photoluminescence (dot, excitation at $300 \mathrm{~nm}$ ) and up-conversion (solid, excitation at $767 \mathrm{~nm}$ ) luminescence of $\mathrm{Mn}^{2+}$ in $\mathrm{ZnS}: \mathrm{Mn}^{2+}$ bulk and nanoparticles. The emission lifetime of the photoluminescence excited at $300 \mathrm{~nm}$ is longer than the up-conversion lifetime excited at $767 \mathrm{~nm}$.

argues that the up-conversion luminescence is derived from a two-photon absorption process.

As revealed by electron spin resonance (ESR), $\mathrm{Mn}^{2+}$ can occupy two sites in $\mathrm{ZnS}: \mathrm{Mn}^{2+}$ nanoparticles: the lattice sites of $\mathrm{Zn}^{2+}$ ions and the near surface sites (Murase et al. 1999; Chen et al. 2000;, Chen et al. 2001). These different sites should display different emission spectra and decay lifetimes. In the nanoparticles, the up-conversion and the $383.5 \mathrm{~nm}$ excited photoluminescence emission maxima shift to lower energies in comparison with the photoluminescence energy when excited at $300 \mathrm{~nm}$, and their decay lifetimes are shorter than the lifetimes obtained with $300 \mathrm{~nm}$ excitation. This could be due to site-selected excitation of $\mathrm{Mn}^{2+}$ ions or size-selected excitation of the nanoparticles. Based on the data obtained, we attribute the up-conversion emission and the photoluminescence emission excited at $383.5 \mathrm{~nm}$ to the $\mathrm{Mn}^{2+}$ at the near surface sites. The $\mathrm{Mn}^{2+}$ ions at the near surface sites have a faster decay lifetime and a lower 
emission energy than the $\mathrm{Mn}^{2+}$ ions at the bulk-like lattice sites. One reason is that the symmetry of the near surface sites is lower than the lattice sites. The excited levels of $\mathrm{Mn}^{2+}$ are split in the near surface sites due to the change in the crystal symmetry. The lowest emitting state will shift to a lower energy position, and, thus, the emission energy is shifted to longer wavelengths. Due to the change in the crystal field symmetry, the transition property of the emitting state is also changed, from forbidden to allowed or partly allowed. This may be one reason why the upconversion and the $383.5 \mathrm{~nm}$ excitation decay lifetimes are shorter than the $300 \mathrm{~nm}$ excitation lifetimes. Another reason is that the coupling of the surface carriers to the near surface site $\mathrm{Mn}^{2+}$ ions is stronger than to the lattice site $\mathrm{Mn}^{2+}$ ions. Thus, more surface carriers are involved in the luminescence process of the near surface site $\mathrm{Mn}^{2+}$ ions. This also may shift the emission to lower energies and shorten the decay lifetime.

There is another possible interpretation of the redshifted photoluminescence (following $383.5 \mathrm{~nm}$ excitation) or up-conversion emission compared to the photoluminescence following excitation at $300 \mathrm{~nm}$. If the $300 \mathrm{~nm}$ excitation preferentially excites smaller particles compared to the $383.5 \mathrm{~nm}$ or $767 \mathrm{~nm}$ (twophoton) excitation, the emission following $300 \mathrm{~nm}$ excitation may have a peak blue-shifted relative to that of the $383.5 \mathrm{~nm}$ or $767 \mathrm{~nm}$ excitation since smaller particles tend to have higher energy absorption and emission compared with larger particles. Further work is necessary to establish which interpretation is correct.

\section{References}

Chen, W., R. Sammynaiken, and Y. Huang, J. Appl. Phys, 88, 5188 (2000).

Chen, W., R. Sammynaiken, Y. Huang, J-O Malm, R. Wallenberg, J-O Bovin, V. Zwiller, and N. A. Kotov, J. Appl. Phys. 89, 1120 (2001).

Cho, Y-H, D. S. Kim, and B-D Choe, Phys. Rev. B 54, R4375 (1997).

Halwa, H. S. (Ed.), Handbook of Nanostructured Materials and Nanotechnology: Optical Properties, Volume 4, Academic Press, 2000.

Lenth, W., and R. M. MacFarlane, Opt. Photonics News 8 (1992).
Murase, N., R. Jagannathan, Y. Kanematsu, M. Watanabe, A. Kurita, K. Hirata, T. Yazawa, and T. Kushida, J. Phys. Chem. B 103, 754 (1999).

Pollnau, M., D. R. Gamielin, S. R. Luthi, H. U. Gudel, and M. P. Hehlen, Phys. Rev. B 61, 3337 (2000).

\section{Vehicle Exhaust Treatment Using Electrical Discharge Methods}

\author{
R. G. Tonkyn, S. E. Barlow, \\ A. Ebeling, C. H. F. Peden. \\ Supported by a Cooperative Research and \\ Development Agreement with the Low \\ Emissions Technology Research and \\ Development Partnership
}

The combination of non-thermal plasma and heterogeneous catalysis is a promising treatment for the abatement of NOx in lean-burn spark ignition and light duty diesel exhaust. Treatment of the oxygen rich exhaust by plasma alone does not result in the chemical reduction of NO. However, we have previously shown that with an appropriate reductant the plasma-treated NOx can be catalytically reduced to nitrogen and nitrous oxide. Increasingly stringent regulations controlling the allowable quantities of NOx emissions from light duty diesel exhaust have recently changed our target for NOx remediation from 50 percent to 90 percent. Although we have found many different catalysts that satisfy the original 50 percent conversion target, none to date has shown the ability to reach 90 percent conversion.

We observe a limiting maximum NOx removal efficiency under a wide variety of reaction conditions and catalysts. Figures 3.11 and 3.12 illustrate this effect with respect to hydrocarbon concentration and energy density. Similar limiting behavior is observed with respect to either the volume of catalyst or the percentage of oxygen in the gas stream. The data shown are with barium $\mathrm{Y}$ zeolite, (Ba-Y) one of our most promising catalysts.

The maximum in NOx reduction is consistent with the following simple two-step mechanism. First, NO is oxidized to $\mathrm{NO}_{2}$ in the gas phase by discharge driven chemistry. During this oxidation partially oxidized hydrocarbons are also formed, which are able to 


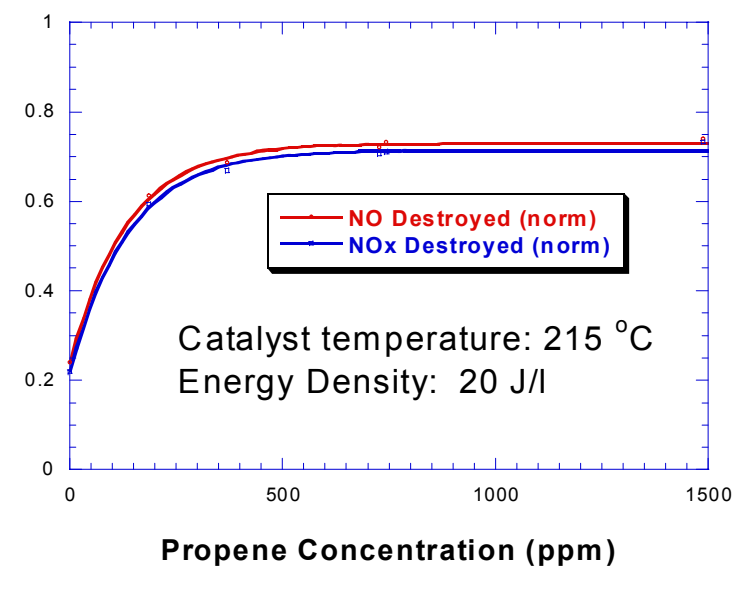

Figure 3.11. Conversion of NOx versus propene concentration over Barium $Y$ zeolite. The data were taken at the optimal temperature with high energy density. The gas included $6 \%$ oxygen, $2 \%$ water, 200 ppm NO, 600 ppm propene in a nitrogen background.

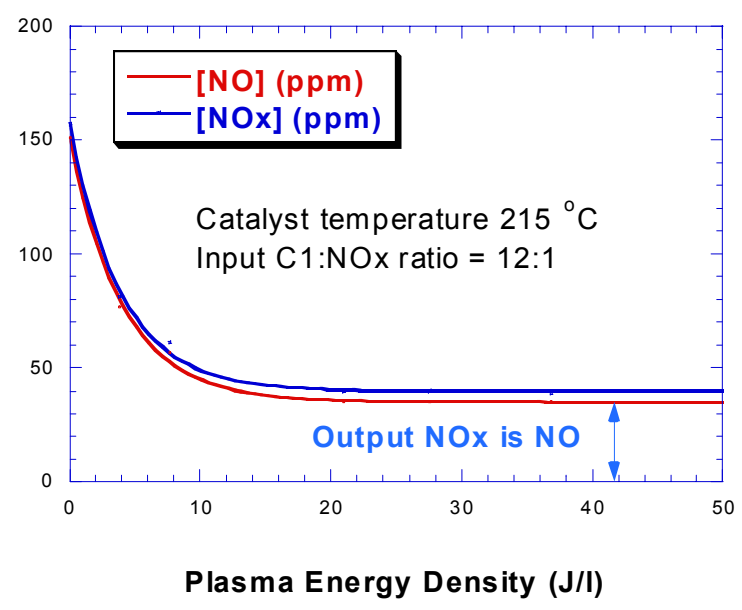

Figure 3.12. Similar behavior is seen versus energy. Note the NOx out is almost entirely made up on NO, even though at energies above a few joules per liter all of the $\mathrm{NO}$ is converted to $\mathrm{NO}_{2}$ in the plasma.

reduce surface bound $\mathrm{NO}_{2} . \mathrm{NO}_{2}$, but not $\mathrm{NO}$, is then reduced on the catalyst by partially oxidized hydrocarbons. The maximum in NOx conversion arises from the non-selective reduction of $\mathrm{NO}_{2}$ to $\mathrm{N}_{2}, \mathrm{~N}_{2} \mathrm{O}$, and $\mathrm{NO}$. Any $\mathrm{NO}_{2}$ back-converted to $\mathrm{NO}$ will appear as NOx in the output stream because NO is unreactive on our catalysts. In other words the maximum obtainable percentage reduction is limited by the catalytic selectivity towards NO. Adding additional hydrocarbon, plasma energy or catalyst will not help, because that fraction of NOx converted to NO is unable to react further. The observation that NO does not adsorb on our zeolite catalysts likely explains this result.

We have found that hydrocarbons are significantly less reactive in the plasma + catalyst system than NO. For modest plasma energy density the final exhaust gas always contains a higher ratio of reductant to NO than the input gas. The fact that the output gas contains NOx as NO while still containing excess reductant suggests that the overall NOx conversion can be improved simply by treating the gas a second time. In this way, we can reconvert the $\mathrm{NO}$ to $\mathrm{NO}_{2}$ while replenishing the supply of partially oxidized hydrocarbons available as reductants. If necessary, further stages of plasma catalyst treatment could be added. We have analyzed the expected return from consecutive stages of plasma + catalyst treatment of NOx in simulated exhaust gas. We have also experimentally verified the concept.

\section{Multi-stage exhaust treatment- theory}

In what follows, we consider a multi-stage reactor where each stage contains a non-thermal plasma followed by a catalyst bed. In each stage of the reactor, the electrical discharge first oxidizes $\mathrm{NO}$ to $\mathrm{NO}_{2}$ and partially oxidizes the hydrocarbons. This mixture is then introduced to a catalyst bed where the $\mathrm{NO}_{2}$ reacts with the partially oxidized hydrocarbons and oxygen by as yet poorly understood mechanisms, to produce molecular nitrogen, water and carbon dioxide. In our experiments we always find that a significant fraction of the $\mathrm{NO}_{2}$ is reduced back to NO. In fact most, if not all, of the output NOx is NO. To achieve nominal target test cycle mass reductions of 90 percent, the output of the catalyst bed must be treated further. This can be done by placing a second stage of the discharge/catalyst reactor after the first. If necessary, a third or fourth stage can be added.

In order to analyze a single stage of a multi-stage reactor, we make the following simplifications. First, the plasma converts $\mathrm{NO}$ only to $\mathrm{NO}_{2}$. In each stage the conversion depends in the same way on the energy density of the plasma, commonly measured in joules per liter of exhaust. Second, NO is completely unreactive over the catalyst. Finally any $\mathrm{NO}_{2}$ which is not reduced to $\mathrm{N}_{2}$ (or $\mathrm{N}_{2} \mathrm{O}$ ) is converted to $\mathrm{NO}$. The final 
assumption simply means that at each stage we supply sufficient catalyst to take the reduction to its limiting value. The mathematics of multi-stage treatment is completely straightforward once a single stage of plasma + catalyst is calculated. In the multistage reactor, the output of the engine, $[\mathrm{NO}]_{0}$ enters the first stage of the reactor; an amount $[\mathrm{NO}]_{1}$ exits it and is fed into the second stage. After the second stage, an amount $[\mathrm{NO}]_{2}$ is left for processing in the third stage and so forth.

Figure 3.13 illustrates the improvement possible in the overall NOx reduction by treating the exhaust successively with plasma + catalyst. Here the value of $\chi$ is the catalyst efficiency for converting $\mathrm{NO}_{2}$ to $\mathrm{N}_{2}$. Clearly, if we have a 90 percent efficient catalyst only one stage is required, and no benefit accrues from multiple stages of treatment. For catalyst efficiencies less than 90 percent not only can we reach the 90 percent target by this method, but we can expect significant energy savings by choosing the correct number of stages.

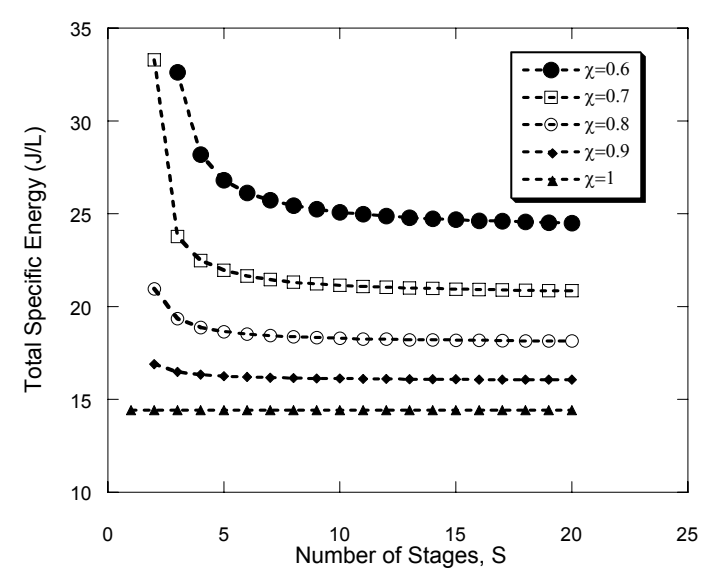

Figure 3.13. The minimum number of stages, $S$, required to reach 90 percent conversion is determined by the absolute catalyst efficiency $\chi$. The total energy required in the plasma drops significantly with increased S, with the gains possible strongly dependent on the catalyst efficiency. The best catalysts to date have a maximum $\chi$ of between 0.6 and 0.7 .

\section{Multi-stage exhaust treatment- experiment}

We have investigated the utility of two-stage plasmacatalysis treatment for NOx abatement in synthetic exhaust. For these experiments, the gas mixture included only six percent oxygen, one percent water,
560 ppm propylene and $180 \mathrm{ppm}$ NO in a nitrogen bath. Two plasma-catalyst reactor systems were run in series, each with $10 \mathrm{cc} \mathrm{Ba-Y}$ extrudates. The gas always passed through both stages, and the plasma energies were independently varied. In this way we could compare one and two-stage reactors at various plasma energies without changing the temperature, gas mixture or catalyst volume. Figure 3.14 illustrates the advantage of adding a second plasma stage. A significant improvement is observed at higher powers when the second plasma is turned on. At very low power, a second plasma stage is somewhat counterproductive. This is easily explained by considering the limit of zero power into the first stage. In this case we have a single plasma-catalyst system with only half as much catalyst. The figure suggests that we might approach the 90 percent target at reasonable energy levels by using one more plasma-catalyst stage.

We have carried out a detailed comparison of one versus two stages of plasma-catalysis as a function of both temperature and hydrocarbon-to-NOx ratio. Figure 3.15 illustrates that the advantages of multiple plasma regions are significant over the entire temperature range. Adding a second stage of plasma improves conversion at 6:1 hydrocarbon to NOx ratio more than increasing the ratio to 9:1. This could be an important tradeoff in efforts to minimize the fuel penalty incurred by plasma-catalysis exhaust treatment.

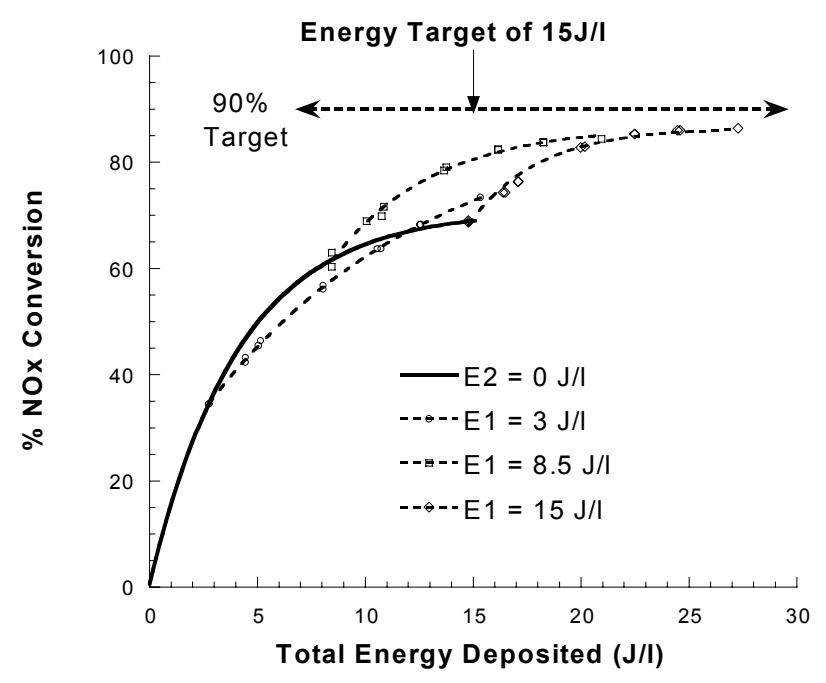

Figure 3.14. Using a second plasma stage to reconvert $\mathrm{NO}$ to $\mathrm{NO}_{2}$ and replenish the supply of partially oxidized hydrocarbon on the catalyst can increase the maximum obtainable NOx conversion. 


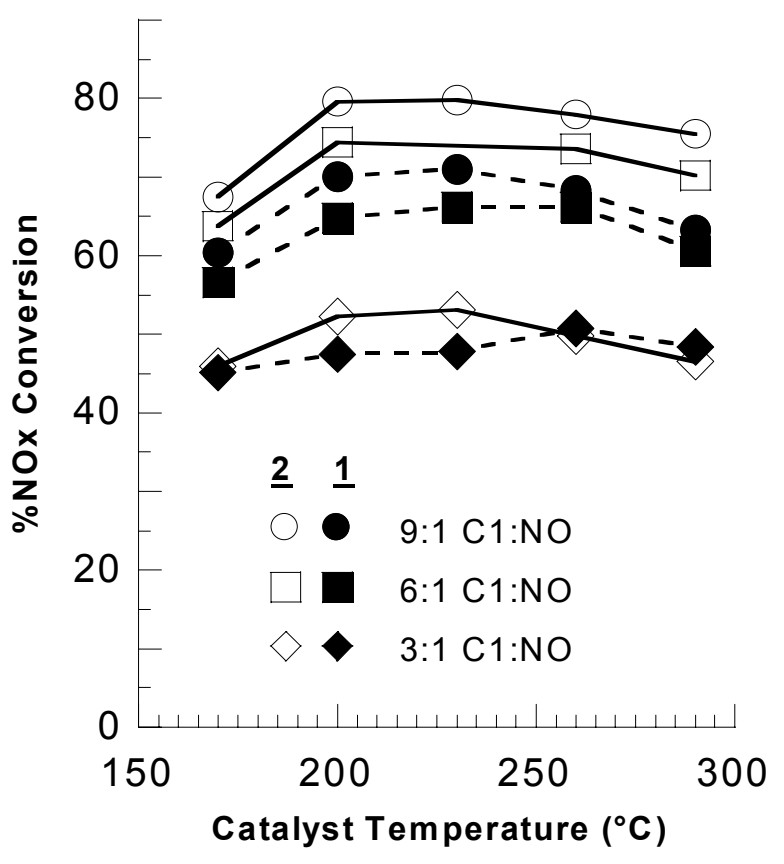

Figure 3.15. Open points indicate both plasma's were turned on; closed points indicate that only the first plasma was on. The total energy deposited was $32 \mathrm{~J} / \mathrm{l}$, with half deposited in each stage when two plasmas were used. The catalyst was barium zeolite $\mathrm{Y}$.

With our present set of zeolite catalysts, the multiple plasma-catalysis treatment strategy may well be the only means of approaching the program goal of 90 percent NOx conversion. Future experiments will include three stage experiments, as well as efforts to optimize the conditions for each stage to maximize NOx conversion while minimizing the overall fuel penalty. Diesel fuel will be substituted for propene as a more realistic possible reductant.

\section{Electron-Stimulated Reactions at Surfaces and Interfaces}

\author{
G. A. Kimmel and N. G. Petrik
}

Supported by BES Chemical Sciences.

\section{Project Overview}

Energetic processes at surfaces and interfaces are important in fields such as radiation chemistry, radiation biology, waste processing, and advanced materials synthesis. Low-energy electrons $(<100 \mathrm{eV})$ frequently play a dominant role in these processes since the higher energy primary particles produce numerous low-energy, chemically active, secondary electrons. In addition, the presence of surfaces or interfaces modifies the physics and chemistry compared to what occurs in the bulk. We use resonance enhanced multi-photon ionization (REMPI) spectroscopy, quadrupole mass spectroscopy, and other ultrahigh vacuum (UHV) surface science techniques to investigate energetic, electron-stimulated reactions at surfaces and interfaces, in nanoscale materials, in thin molecular solids, and in biomolecules. A key element of the approach is to use a tunable, mono-energetic electron beam to unravel the complex chemistry that results from the simultaneous release of electrons with a broad range of energies by the primary excitation events associated with ionizing radiation. This work addresses several important issues, including understanding the relative importance of the primary excitation source compared to the secondary electrons it produces, energy transfer at surfaces and interfaces, and new reaction pathways at surfaces.

\section{Electron-stimulated reactions in amorphous solid water}

We are investigating the electron-stimulated production of neutral atomic and molecular products from thin films of amorphous solid water (ASW) and crystalline ice. Our results indicate that the reactions occur primarily at either the substrate/water interface or the water/vacuum interface. However, the energy that drives the reactions is deposited primarily in the bulk of the film and subsequently diffuses to the interfaces.

\section{Motivation and Background}

The structure of condensed water (Mayer and Pletze 1986; Jenniskens and Blake 1994; Stevenson et al. 1999) and its interactions with electrons (Rowntree et al. 1991; Noell et al. 1985; Kimmell, et al. 1994, 1995, 1996, 1997), photons (Kouchi and Kuroda 1990; Westley et al. 1995), and ions (Kouchi and Kuroda 1990; Westley et al. 1995) have been extensively studied due to water's importance in scientific disciplines as diverse as radiation biology and astrophysics. Stimulated reactions in ASW and crystalline ice lead to the formation of a variety of products such as anions $\left(\mathrm{H}^{-}\right)$, neutral atoms $(\mathrm{O}, \mathrm{H})$, radicals $(\mathrm{OH})$ and molecules $\left(\mathrm{H}_{2}, \mathrm{O}_{2}\right)$. Electronically excited states, dissociative electron attachment, electron-ion 
recombination and exciton dissociation have all been implicated in stimulated reactions in water. In addition to initiating chemical reactions, energetic particles can induce changes in the structure of the water films. For example, a recent report indicates that lowenergy electrons can induce crystallization of thin ASW films (Chakarov and Kasems 1998).

Despite the studies mentioned above, important aspects of the interaction of electrons with crystalline ice and ASW remain unclear. For example, while dissociative electron attachment is known to occur on the surface of thin water films, its role in the bulk is not understood. In fact, the relative importance of surface and bulk reactions is not well known for any electron-stimulated reaction. More generally, there is no consensus regarding the mechanisms of nonthermal, electron-stimulated reactions in molecular solids.

One approach for investigating the role of surface and bulk reactions in ASW is to grow layered films using $\mathrm{H}_{2} \mathrm{O}$ and $\mathrm{D}_{2} \mathrm{O}$ and then measure the relative yields of the hydrogen containing products. For example, lowenergy electrons $(10-100 \mathrm{eV})$ induce electronic excitations that lead to the formation and desorption of neutral hydrogen atoms and other products. By studying the electron stimulated desorption yield from $\mathrm{H}_{2} \mathrm{O}$ films as a function of $\mathrm{D}_{2} \mathrm{O}$ coverage, we can investigate the depth at which the hydrogen containing reaction products (e.g. $\mathrm{H}, \mathrm{OH}, \mathrm{H}_{2}$ ) were formed. Isotopic labeling with $\mathrm{H}_{2} \mathrm{O}^{18}$ can also be used to investigate the formation of products involving oxygen.

The diffusion of electronic excitations, atoms and molecules within ASW and crystalline ice is of interest to astrophysicists since it is important for the formation of molecules on grains within interstellar dust clouds. Diffusion is also critical for understanding the product yields observed in the radiolysis of water-a subject of great interest in radiation biology. Studies using isotopically labeled layers will also allow us to investigate the diffusion kinetics of atoms $(\mathrm{H}$ and $\mathrm{O})$ and molecules $\left(\mathrm{H}_{2}\right)$ formed by energetic electrons in the ices. Preliminary data (discussed below) indicate that $\mathrm{D}_{2}$ molecules formed below the surface during electron irradiation of $\mathrm{D}_{2} \mathrm{O}$, which subsequently diffuse to the surface of the film, are a significant fraction of the total electron-stimulated production of $\mathrm{D}_{2}$. Additionally, these preliminary studies indicate that the diffusion of excitons in the ASW films is also important in electron-stimulated reactions.

\section{Experimental Approach}

The experiments are performed in two ultra-high vacuum (base pressure $\sim 1 \times 10^{-10}$ torr) systems. System I is currently operational and System II is being developed. System I is equipped with a temperaturecontrolled ( $\sim 90 \mathrm{~K}-1500 \mathrm{~K})$ 4-axis sample manipulator, a quadrupole mass spectrometer, a time-of-flight (TOF) mass spectrometer, and a sputter gun.

System II is equipped with a temperature-controlled ( $20 \mathrm{~K}-1500 \mathrm{~K})$ 4-axis sample manipulator, a quadrupole mass spectrometer, a TOF mass spectrometer, an auger electron spectrometer, a sputter gun, thin film evaporators, and a molecular beam doser. Each system is equipped with a low-energy electron gun (Kimball Physics ELG-2) that produces a monoenergetic (FWHM $\sim 0.3 \mathrm{eV})$, tunable $(\sim 2 \mathrm{eV}-1000 \mathrm{eV})$ electron beam. In both systems, thin films with controlled coverages (submonolayer to multilayers) of the target molecules can be adsorbed on atomically clean, well-characterized surfaces. Accurate coverage calibrations are achieved using temperature programmed desorption (TPD) techniques. The neutral desorbates are detected using quadrupole mass spectroscopy and/or REMPI-TOF spectroscopy. REMPI is a sensitive, quantum-state-specific detection technique which allows for complete characterization of the energy partitioning (electronic, translation, vibration, and rotation) of the desorption products. The frequencies necessary for REMPI detection are generated by nonlinear frequency conversion of the output of a pulsed, Nd:YAG-pumped, tunable dye-laser. The entire experiment is computer controlled with a versatile, custom software package developed by the Instrument Design Laboratory at PNNL.

\section{Results}

We are currently investigating the electron-stimulated production of $\mathrm{D}, \mathrm{D}_{2}$ and $\mathrm{O}_{2}$ from thin films of ASW and crystalline ice deposited on a Pt(111) substrate using System I. Thin films (thicknesses less than $\sim 400 \mathrm{ML}$ ) of water are deposited on an $\mathrm{LN}_{2}$ cooled $\operatorname{Pt}(111)$ crystal. In these experiments, the films are then irradiated with relatively long duration pulses ( $\sim 1-40$ seconds) of low-energy electrons and the neutral desorbates are detected with a quadrupole mass spectrometer. For most of these experiments, the 
incident electron energy, $E_{i}$, is $100 \mathrm{eV}$ and the sample temperature, $\mathrm{T}_{\mathrm{s}}$, is $100 \mathrm{~K}$.

Figure 3.16 shows the $\mathrm{D}_{2}$ electron-stimulated desorption (ESD) signal as a function of time for a $40 \mathrm{~s}$ long pulse of $100 \mathrm{eV}$ electrons incident on a) $210 \mathrm{ML}$ and b) $70 \mathrm{ML}$ films of $\mathrm{D}_{2} \mathrm{O}$ ASW. For the $210 \mathrm{ML}$ film, the $\mathrm{D}_{2}$ signal is approximately constant during the electron beam pulse. In contrast, for the $70 \mathrm{ML}$ film, there is a prompt increase in the $\mathrm{D}_{2}$ signal when the beam turns on followed by a gradual increase during the pulse duration. After the incident electron pulse ends, the $\mathrm{D}_{2}$ signal decreases approximately exponentially with a time constant of several seconds. Below, we will present evidence that there are two components to the $\mathrm{D}_{2}$ signal measured in these experiments. The first component, as highlighted in Figure 3.16a, is a "prompt" component (i.e., $\mathrm{D}_{2}$, which is only present when the electron beam is on and which is approximately constant versus time). For the $70 \mathrm{ML}$ film, in addition to the "prompt" component, there is a second ("delayed") component that varies with time and can be present after the electron beam irradiation has ended (Figure 3.16b). We will argue that the prompt component is due to reactions that occur at the ASW/vacuum interface and that the delayed component is due to reactions at the Pt/ASW interface.

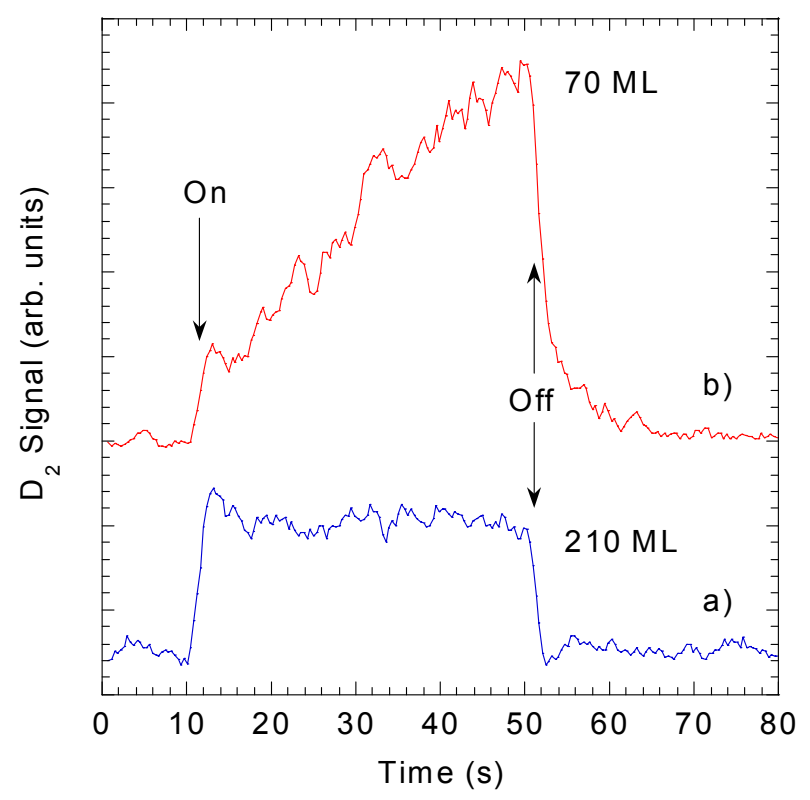

Figure 3.16. $D_{2} E S D$ from $\mathrm{Pt}(111)$ vs. time $\mathrm{E}_{\mathrm{i}}=100 \mathrm{eV}, \mathrm{Ts}=100 \mathrm{~K}$. a) $210 \mathrm{ML} \mathrm{D}_{2} \mathrm{O}$; b) $70 \mathrm{ML} \mathrm{D}_{2} \mathrm{O}$.
The integrated $\mathrm{D}_{2}$ yield versus film thickness for ASW films deposited at $100 \mathrm{~K}$ is shown in Figure 3.17. The electron beam energy is $100 \mathrm{eV}$. In Figure 3.17a (3.17b) the beam pulse duration is 20 (40) seconds. Figure 3.17 a shows the $\mathrm{D}_{2}$ yield integrated over the entire electron pulse - including the $\mathrm{D}_{2}$ which desorbs after the pulse ends. The signal increases rapidly at low coverages, has a maximum at $\sim 50 \mathrm{ML}$, and is constant for coverages greater than $\sim 200 \mathrm{ML}$. In Figure $3.17 \mathrm{~b}$, the yield integrated over 2 second "windows" during (and after) the 40 second electron pulse is plotted versus film coverage. For 0 to $2 \mathrm{~s}$, which is representative of the "prompt" signal, the $\mathrm{D}_{2}$ yield
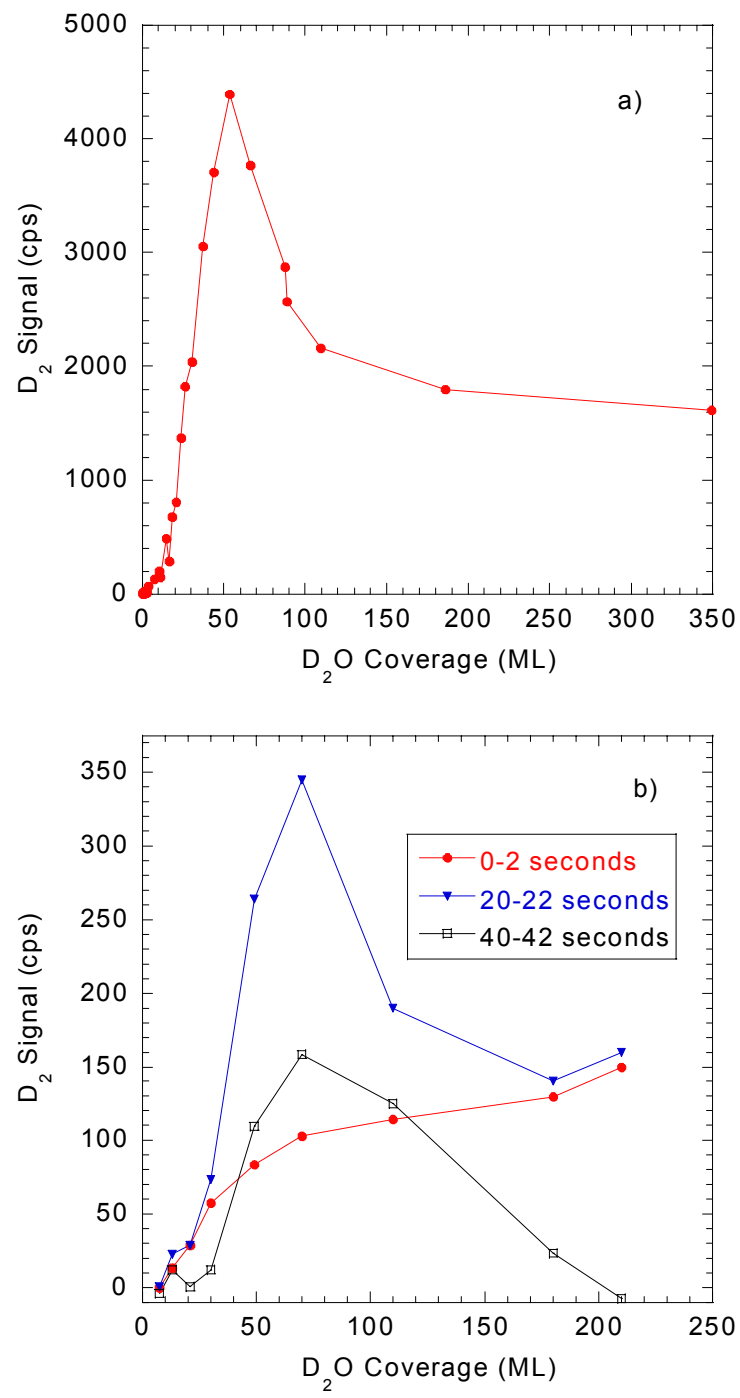

Figure 3.17. a) $D_{2}$ ESD vs. $D_{2} O$ coverage. b) $D_{2}$ ESD vs. $D_{2} \mathrm{O}$ coverage. 
increases monotonically, becoming constant for coverages above $\sim 200 \mathrm{ML}$. For 40 to 42 s, (i.e. after the beam is off), the signal has a maximum at $\sim 70 \mathrm{ML}$ and vanishes for coverages greater than $\sim 200 \mathrm{ML}$. This behavior is characteristic of the "delayed" component. During the middle of the 40 second beam pulse (e.g., 20-22 s), the signal has contributions from both components.

We are using isotopically layered ASW films to investigate the thickness dependence of the electronstimulated production of $\mathrm{D}_{2}$. The $\mathrm{D}_{2}$ yield versus time during irradiation with $100 \mathrm{eV}$ electrons at $\mathrm{T}_{\mathrm{s}}=100 \mathrm{~K}$ is shown for two films in Figure 3.18. In

Figure $3.18 \mathrm{a}$, the $82 \mathrm{ML}$ ASW film is pure $\mathrm{D}_{2} \mathrm{O}$ and the $\mathrm{D}_{2}$ yield versus time has the characteristic shape discussed above (Figure 3.17b). In Figure 3.18b, 5$10 \mathrm{~mL}$ of $\mathrm{H}_{2} \mathrm{O}$ was deposited on the $\mathrm{Pt}(111)$ followed by $\sim 66 \mathrm{ML}$ of $\mathrm{D}_{2} \mathrm{O}$. In this case, the $\mathrm{D}_{2}$ yield, is dramatically reduced, is approximately constant, and is only appreciable during the electron beam pulse, i.e., only the "prompt" component remains. These data suggest that the "delayed" $\mathrm{D}_{2}$ yield is due to reactions in the vicinity (first several ML) of the $\mathrm{Pt}(111)$ surface. The $\mathrm{D}_{2}$ formed near the Pt/ASW interface subsequently diffuses through the ASW film and desorbs. It is this diffusion that is responsible for the $\mathrm{D}_{2}$ observed after the end of electron beam pulse (see Figure 3.16a and Figure 3.18b).

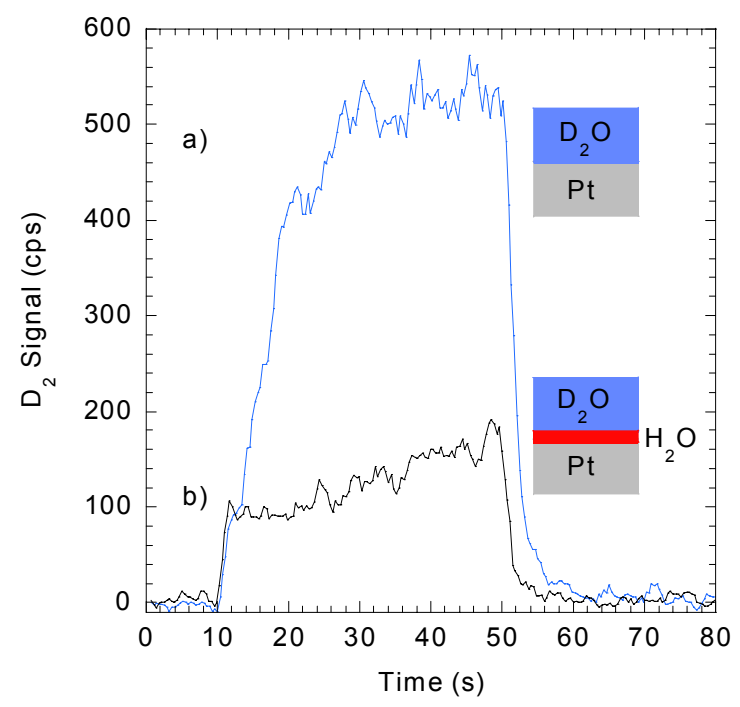

Figure 3.18. $D_{2} E S D$ vs. time. $E_{i}=100 \mathrm{eV}, T_{s}=$ $100 \mathrm{~K}$. a) $\sim 82 \mathrm{ML} \mathrm{D}_{2} \mathrm{O}$ on $\mathrm{Pt}(111)$. B) $\sim 66 \mathrm{ML} \mathrm{D}_{2} \mathrm{O}$ and $\sim 10 \mathrm{ML} \mathrm{H}_{2} \mathrm{O} \mathrm{Pt}(111)$.
Figure 3.19 demonstrates what happens when an $\mathrm{H}_{2} \mathrm{O}$ layer is deposited on top of the $\mathrm{D}_{2} \mathrm{O}$ film. $\mathrm{D}_{2}$ yield versus time from a neat, $\sim 37 \mathrm{ML}$ film of $\mathrm{D}_{2} \mathrm{O}$ is shown in a). $\mathrm{D}_{2}$ yield versus time for $37 \mathrm{ML}$ film of $\mathrm{D}_{2} \mathrm{O}$ capped with $\sim 15 \mathrm{ML}$ of $\mathrm{H}_{2} \mathrm{O}$ is shown in b). In this case, the "prompt" component is suppressed, while the "delayed" component survives, suggesting that the "prompt" $\mathrm{D}_{2}$ component is due to reactions that occur at or near the ASW/vacuum interface. Since it is clear that $\mathrm{D}_{2}$ readily diffuses in ASW at $100 \mathrm{~K}$, the data in Figure 3.18 and 3.19 indicate that if appreciable amounts of $\mathrm{D}_{2}$ were formed in the bulk of the ASW films (i.e., not at one of the interfaces) it would be observable. Its absence (Figure 3.18 ) suggests that relatively little $\mathrm{D}_{2}$ is formed in the bulk.

While the isotopic labeling suggests that the reactions that lead to $\mathrm{D}_{2}$ occur at or near the interfaces, the yield versus film thickness suggest that the energy for the reactions is deposited in the bulk of the film. For the delayed component, which apparently emanates from the first few monolayers near the Pt/ASW interface, the yield reaches a maximum only after $\sim 70 \mathrm{ML}$. Likewise the prompt component, which results from dissociations at or near the ASW/vacuum interface, does not reach its maximum value until the film is more than $100 \mathrm{ML}$ thick. The isotopic labeling experiments also suggest that radicals that might be created in the bulk and that might subsequently

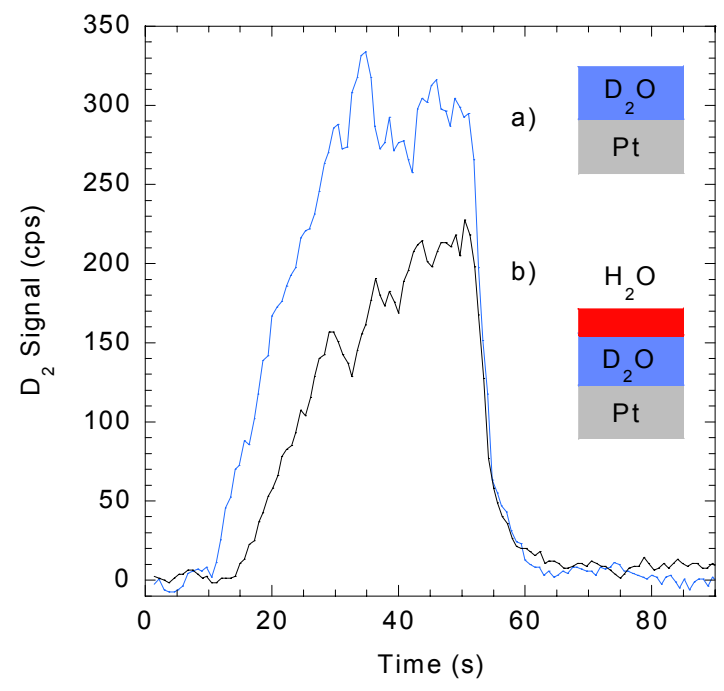

Figure 3.19. $D_{2} E S D$ vs. time. $E_{i}=100 \mathrm{eV}, T_{s}=$ $100 \mathrm{~K}$. a) $\sim 37 \mathrm{ML} \mathrm{D}_{2} \mathrm{O}$ on $\mathrm{Pt}(111)$. b) $\sim 15 \mathrm{ML} \mathrm{H}_{2} \mathrm{O}$ and $\sim 37 \mathrm{ML} \mathrm{D}_{2} \mathrm{O}$ on $\mathrm{Pt}(111)$. 
diffuse to the interfaces are not contributing to the observed $\mathrm{D}_{2}$ yield (the yield of $\mathrm{HD}$ also supports this conclusion). However, ionizations and excitations that lead to the formation of excitons in the bulk might be responsible for the thickness dependence of the $\mathrm{D}_{2}$ yield. In this picture, excitons formed throughout the film are highly mobile and can rapidly diffuse to the interfaces. For thin films, the yield of $\mathrm{D}_{2}$ from both interfaces is limited by the number excitations in the film. For thicknesses that are less than the exciton diffusion length scale and the incident electron penetration depth, increasing the film thickness increases the $\mathrm{D}_{2}$ yield. Some of our previous investigations of the ESD of $\mathrm{D}$ and $\mathrm{O}$ atoms from $\mathrm{D}_{2} \mathrm{O}$ films support this picture of exciton diffusion (Orlando and Kimmel 1997).

For coverages greater than $\sim 200 \mathrm{ML}$, there is apparently no $\mathrm{D}_{2}$ produced at the $\mathrm{Pt}(111) / \mathrm{ASW}$ interface suggesting that the electron beam penetration depth at $100 \mathrm{eV}$ is less than the thickness of the films. For these thicker films, since excitons are still being formed in the outer layers by the incident electron beam, the lack of $D_{2}$ production also suggests that the film thickness is greater than the exciton diffusion length. For $\mathrm{D}_{2}$ produced at the ASW/vacuum interface, the yield eventually saturates for thicker films. In this case, the saturation may be due to the limited diffusion length of the excitons or the penetration depth of the incident electrons. Unfortunately, while electron scattering cross-sections have been measured in ASW films at low energy (1-20 eV) (Michaud and Sanche 1987), experimental scattering cross-sections are not available for higher energies (Pimpblott and Mozumder 1991).

Apparently, electronic excitations do not lead to significant production of $\mathrm{D}_{2}$ in the bulk. This could be due to a number of reasons. For example, it could be that "cage" effects in the bulk result in rapid and efficient back reactions, limiting the $\mathrm{D}_{2}$ yield. Alternatively, if the exciton is highly mobile, it might hop from site to site on a time scale that is short compared to the dissociation time for reaction. In that case, the interface might act as a trap for the exciton, allowing dissociation to proceed.
We have also measured the yields of $\mathrm{D}$ and $\mathrm{O}_{2}$ versus film thickness. The dependence of the yields of these species on film thickness is qualitatively similar to that found for $\mathrm{D}_{2}$ (data not shown). However, we do not yet know if the $\mathrm{D}$ and $\mathrm{O}_{2}$ are also produced primarily at the ASW interfaces. In general, all the results discussed in this section are preliminary in nature. As a result, data interpretations are also preliminary. Our primary short-term objective is to address in detail the issues raised by these experiments.

\section{References}

Chakarov, D. and B. Kasemo, Physical Review Letters 81, 5181 (1998).

Jenniskens, P. and D. F. Blake Scienc. 265, 753 (1994).

Kimmel, G. A. and T. M. Orlando, Physical Review Letters 75, 3983 (1996).

Kimmel, G. A. and T. M. Orlando, Physical Review Letters 75, (13) 2606-2609 (1995).

Kimmel, G. A., et al., J. Chem. Phys. 101, 3282-3286 (1994).

Kimmel, G. A., et al., Journal of Physical Chemistry, B 101, 6301 (1997).

Kimmel, G.A., R. G. Tonkyn, and T. M. Orlando, Nuclear Instruments and Methods in Physics Research, B 101, 179 (1995).

Kouchi, A. and T. Kuroda, Nature 344, 134 (1990). Mayer, E. and R. Pletzer, Nature 319, 298 (1986).

Michaud, M. and L. Sanche, Physical Review A 36, 4672-4683 (1987).

Noell, J. O., C.F. Melius, and R. H. Stulen, Surface Science 157, 119-150 (1985).

Orlando, T. M. and G. A. Kimmel, Surface Science 390, 79 (1997).

Pimblott, S. M. and A. Mozumder, J. Phys. Chem. 95, 7291-7300 (1991).

Rowntree, P., L. Parenteau, and L. Sanche, J. Chem. Phys. 95, (15) 8570-8576 (1991).

Stevenson, K. P., et al. Science 283, 1505 (1999).

Westley, M. S., et al. Nature 373, 405 (1995). 


\section{XAFS Study of Rare Earth Element Coordination in Calcite}

\author{
E. J. Elzinga, ${ }^{(a)}$ R. J. Reeder, ${ }^{(a)}$ \\ S. H. Withers, ${ }^{(b)}$ R. E. Peale, ${ }^{(b)}$ \\ R.A.Mason, ${ }^{(c)}$ K. M. Beck, ${ }^{(d)}$ and \\ W.P. Hess ${ }^{(d)}$
}

This work was funding by the Environmental

Management Science Program of DOE.

Drs Beck and Hess were also supported by the

Divisions of Chemical Sciences of the Office of

Basic Energy Sciences.

(a) Department of Geosciences, State University of New York at Stony Brook.

(b) Department of Physics, University of Central Florida.

(c) Department of Earth Sciences, Memorial University of Newfoundland, St. John's, NF, Canada.

(d) Pacific Northwest National Laboratory.

Rare earth element (REE) patterns have long been recognized as valuable indicators of provenance and alteration for minerals and rocks. Ca-bearing minerals are commonly enriched in REEs relative to other phases, reflecting the ease with which REEs substitute for $\mathrm{Ca}$. The REEs usually occur as trivalent ions and show similar chemical behaviors owing to their electronic configurations. A notable difference among the trivalent ions is a decrease in ionic radius with increasing atomic number, known as the lanthanide contraction. The size range covered by the trivalent REEs encompasses that of $\mathrm{Ca}$, and this similarity is one reason for the compatibility of trivalent REEs with Ca-bearing phases, despite the difference in charge. Relatively few studies have addressed the incorporation of REEs into calcium carbonate, despite the widespread occurrence of calcite and aragonite in sediments and sedimentary rocks. More recently, interest in trivalent REEs in carbonate systems has developed because of expected similarities with the chemical behavior of trivalent actinide species (e.g., $\mathrm{Am}^{3+}, \mathrm{Cm}^{3+}, \mathrm{Pu}^{3+}$ ), which pose serious threats as contaminants associated with radionuclide waste sources. The use of REEs as analogs avoids some of the difficulties associated with handling actinides in experiments. Uptake by calcite and other carbonates may influence the mobility and fate of actinides in the near-surface environment.
Experimental and field studies have shown that REEs are strongly partitioned into calcite during growth and it has become clear that REEs are effectively scavenged from solutions in which calcite is precipitating, in even small quantities. Trivalent actinides may be similarly taken up by calcite.

A fundamental question for any heterovalent substitution is the charge compensation mechanism. Zhong and Mucci (1995) observed that $\mathrm{Na}^{+}$co-precipitation (from seawater-like solutions) was linearly correlated with REE co-precipitation. On this basis, they argued that REEs substitute in the Ca site and that coprecipitation of $\mathrm{Na}^{+}$may compensate for the trivalent charge of the REE. Presumably that would be effective only if $\mathrm{Na}^{+}$or another monovalent ion were a near neighbor of the $\mathrm{REE}^{3+}$ and also substituting for a $\mathrm{Ca}^{2+}$. However, it is also possible that the local coordination of the REE is affected in other ways.

Our focus in the present study is the structural environment of REEs in dilute calcite solid solutions. We use X-ray absorption fine structure (XAFS) spectroscopy to examine the local coordination of selected $\mathrm{REE}^{3+}$ co-precipitated with calcite. We considered $\mathrm{Nd}, \mathrm{Sm}, \mathrm{Dy}$, and $\mathrm{Yb}$, a choice partly constrained by practical considerations but nevertheless spanning a significant portion of the lanthanide series. Because concentrations of REEs in natural calcite are generally too low for XAFS study, we have synthesized calcites with higher concentration of the REEs of interest. Our results confirm that REEs occupy the Ca site as expected, but also indicate that the REEs Nd and Sm adopt a different local coordination than Dy and $\mathrm{Yb}$. This difference should be reflected in the stabilities of the dilute solid solutions and perhaps in their ability to retain the lanthanide impurity.

The near-edge regions of the normalized L3-edge absorption spectra are shown in Figure 3.17. The REE-doped calcite spectra are generally similar in appearance, with all showing a characteristic intense white line feature. The REE-doped calcite spectra differ in detail from the spectra of the corresponding rare-earth carbonates and also from the REE oxides. There is no evidence of features in any of the spectra attributable to multi-electron excitations such as those described by Solera et al. (1995) and D'Angelo et al. (2001). Fourier transform magnitudes of all calcite:REE spectra (Figure 3.21.) are dominated by a single low-R peak attributable to the first shell of 


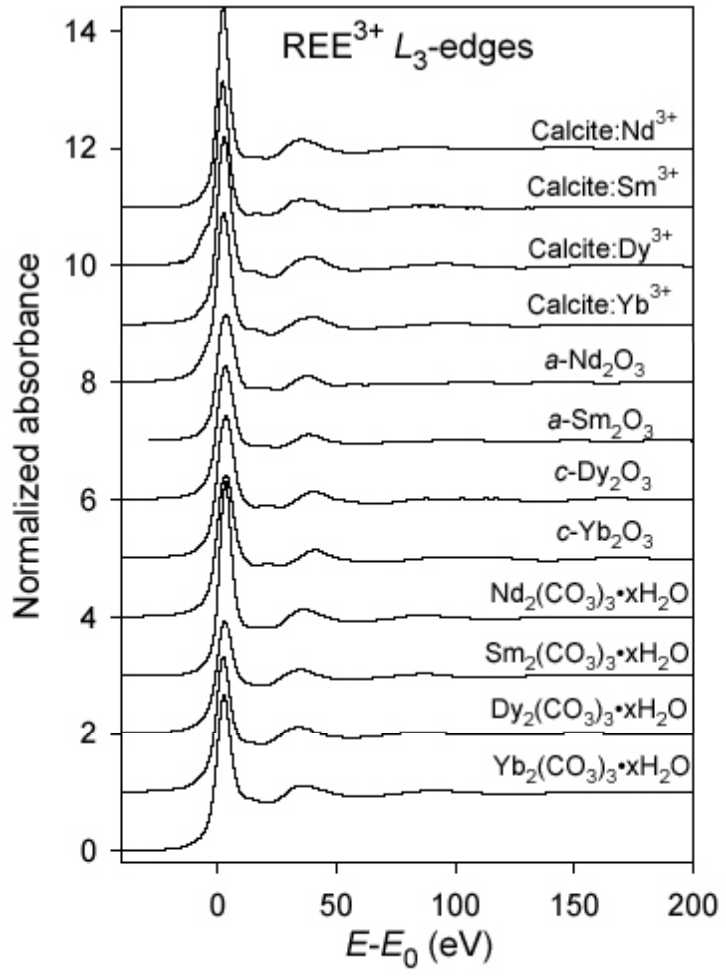

Figure 3.20. The near-edge regions of the normalized REE L3-edge absorption spectra of Nd-, $\mathrm{Sm}-$, Dy-, and $\mathrm{Yb}$-doped calcite. Also shown are the L3-edge absorption spectra of solid $\mathrm{REE}_{2} \mathrm{O}_{3}$ and $\mathrm{REE}_{2}\left(\mathrm{CO}_{3}\right)_{3} \cdot \mathrm{xH}_{2} \mathrm{O}$ reference standards $(\mathrm{REE}=\mathrm{Nd}$, $\mathrm{Sm}, \mathrm{Dy}$, and $\mathrm{Yb}$ ).

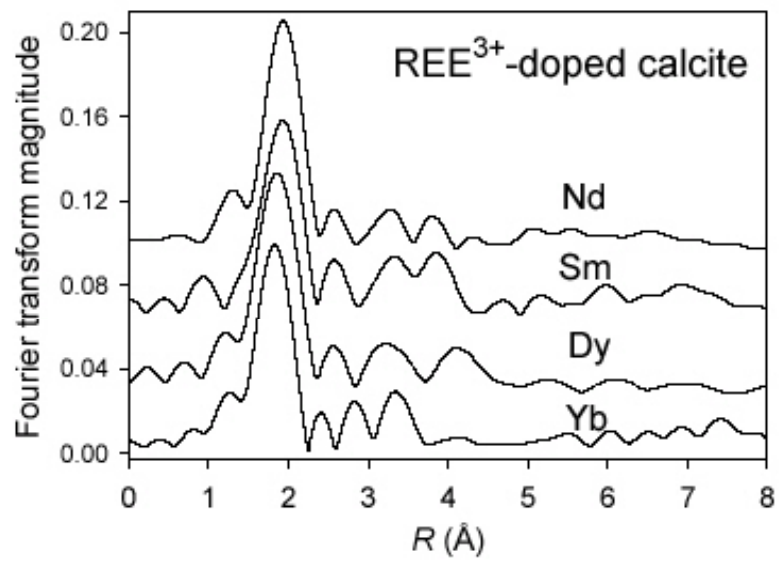

Figure 3.21. Fourier transform (FT) magnitudes (not corrected for phase shifts) of the REE ${ }^{3+}$-doped calcites.

oxygen atoms. Smaller peaks at higher $\mathrm{R}$ are consistent with shells from $\mathrm{C}, \mathrm{O}$, and $\mathrm{Ca}$, consistent with occupation of the REE at the unique Ca site. Initial fitting used paths calculated for the ideal calcite structure using FEFF7. Best-fit parameters using these paths are given in Table 3.1.

The observed first-shell REE-O distances decrease systematically across the series, being $2.41,2.38$, 2.30, and $2.24 \AA$ for $\mathrm{Nd}, \mathrm{Sm}, \mathrm{Dy}$, and $\mathrm{Yb}$, respectively. This trend is consistent with the decrease in

\begin{tabular}{|c|c|c|c|c|c|c|}
\hline \multirow[t]{2}{*}{ REE } & \multicolumn{4}{|c|}{ REE-O1 } & \multicolumn{2}{|c|}{ REE-C } \\
\hline & $N$ & $R(\AA)$ & ó $2(\AA ̊ 2)$ & $N^{*}$ & $R(\AA)$ & ó $2(\AA 2)$ \\
\hline $\mathrm{Nd}$ & 9.5 & 2.41 & 0.008 & 6 & 3.26 & 0.018 \\
\hline $\mathrm{Sm}$ & 8.3 & 2.38 & 0009 & 6 & 3.24 & 0.008 \\
\hline Dy & 7.9 & 2.30 & 0.007 & 6 & 3.21 & 0.006 \\
\hline \multirow[t]{3}{*}{$\mathrm{Yb}$} & 7.5 & 2.24 & 0.008 & 6 & 3.14 & 0.010 \\
\hline & \multicolumn{4}{|c|}{ REE-02 } & \multicolumn{2}{|c|}{ REE-Ca } \\
\hline & $N$ & $R(\AA)$ & ó $2(\AA 22)$ & $N^{*}$ & $R(\AA)$ & ó $2(\AA 2)$ \\
\hline $\mathrm{Nd}$ & 6 & 3.55 & 0.012 & 6 & 4.13 & 0.022 \\
\hline Sm & 6 & 3.48 & 0.012 & 6 & 4.09 & 0.012 \\
\hline Dy & 6 & 3.43 & 0.006 & 6 & 4.06 & 0.020 \\
\hline $\mathrm{Yb}$ & 6 & 3.27 & 0.011 & 6 & 4.04 & 0.015 \\
\hline
\end{tabular}

Notes: Estimated errors for coordination number $(\mathrm{N})$ are $\pm 20 \%$, for $R \pm 0.02$ $\AA$, and for ó $2 \pm 0.002 \AA 2 . * N$ fixed at value in ideal structure. 
REE3 ${ }^{+}$ionic radii from the light to the heavy ends of the series known as the lanthanide contraction. Fitted coordination numbers range from 7.5 to 9.5 , which are larger than would be expected for occupation of the octahedral Ca site. As we note later, the first-shell REE-O distances, on closer inspection, suggest differences in coordination among these lanthanide ions in calcite.

In the ideal calcite structure, the shells beyond the first oxygen coordination include carbon (at $3.21 \AA$ ), a second oxygen shell (at $3.46 \AA$ ), and calcium (at $4.05 \AA$ ), each having ideal $\mathrm{N}$ values of six

(Figure. 3.22). The Ca shell corresponds to the centers of the octahedra that share corners with the absorber octahedron. A more distant $\mathrm{Ca}$ shell exists at $4.99 \AA$ but was not considered. On the assumption that the local structure is largely like that in ideal calcite, except for localized expansion or contraction, coordination numbers for the carbon, second oxygen, and calcium shells were fixed at 6 ; this reduced the number of fit parameters needed. Fit quality using these four shells was visually good but some discrepancies were evident beyond the Ca shell. Best fit REE-X distances (Table 3.1) for the carbon, second oxygen, and calcium shells also show a decrease from $\mathrm{Nd}$ to $\mathrm{Yb}$, as would be expected for decreasing ionic radius. The REE-Ca distance varies systematically from 4.13 to $4.04 \AA$ for $\mathrm{Nd}$ through $\mathrm{Yb}$. These distances are very near or just slightly larger than the $\mathrm{Ca}-\mathrm{Ca}$ distance in ideal calcite (4.05 $\AA$ ). This confirms that the REEs occupy the Ca position and is

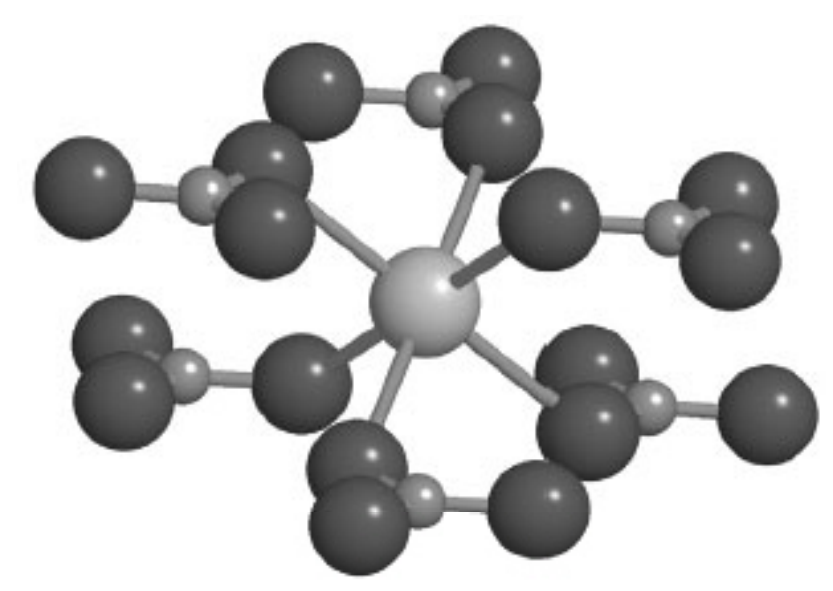

Figure 3.22. Schematic model of the calcite structure showing the octahedral coordination of the Ca site. consistent with previous observations for divalent metal substitution that the $\mathrm{M}^{2+}$-Ca distance deviates only slightly from the ideal value.

The XAFS fit results, accounting for oxygen, carbon, a second oxygen, and calcium shells, clearly support the occupation of the $\mathrm{Nd}^{3+}, \mathrm{Sm}^{3+}, \mathrm{Dy}^{3+}$, and $\mathrm{Yb}^{3+}$ dopants at the Ca site in calcite, as expected. The findings for divalent metal substitution in calcite. Closer inspection of the first-shell oxygen distances, however, reveal some unexpected trends. The Nd-O distance in calcite: $\mathrm{Nd}(2.41 \AA)$ is $0.05 \AA$ greater than the $\mathrm{Ca}-\mathrm{O}$ distance in ideal calcite $(2.36 \AA)$, yet the six-fold ionic radii of $\mathrm{Nd}^{3+}$ and $\mathrm{Ca}^{2+}$ are nearly identical (0.983 and $1.00 \AA$ [Shannon 1976]). This difference of $0.05 \AA$ is greater than the associated estimated errors. The Sm-O distance in calcite: $\operatorname{Sm}(2.38 \AA)$ is also slightly larger than the $2.36 \AA$ $\mathrm{Ca}-\mathrm{O}$ distance in calcite, yet the six-fold ionic radius of $\mathrm{Sm}^{+}(0.958 \AA)$ is smaller than that of $\mathrm{Ca}^{+}(1.00$ $\AA)$. In contrast, the $\mathrm{Dy}-\mathrm{O}$ and $\mathrm{Yb}-\mathrm{O}$ distances in calcite:Dy and calcite: $\mathrm{Yb}$ (2.30 and $2.24 \AA$, respectively) are proportionately smaller than the $2.36 \AA$ Ca-O distance, as would be expected from their smaller ionic radii (Dy $3^{+} 0.912 \AA ; \mathrm{Yb} 3^{+} 0.868 \AA$; [Shannon 1976]). Thus, the Nd-O and Sm-O distances appear anomalously large for the Ca site whereas the Dy-O and $\mathrm{Yb}-\mathrm{O}$ distances appear reasonable. REEs oxygen coordinations in calcite possibly reflects differences in the mechanism of charge compensation when substituting trivalent REEs for divalent $\mathrm{Ca}$ in the calcite structure, and may affect the relative stability of REEcalcite solid solutions.

Taken as a single trend, the observed REE-O distances do not seem to fit either trend line calculated from the six-fold and the seven-fold ionic radii from Shannon (1976). This emphasizes the previous observation, that the $\mathrm{Nd}-\mathrm{O}$ and $\mathrm{Sm}-\mathrm{O}$ distances in calcite are longer than would be expected for six-fold coordination. Yet the Dy-O and $\mathrm{Yb}-\mathrm{O}$ distances are consistent with sixfold coordination. Because the XAFS fits confirm that all four REEs occupy the Ca site, the most obvious interpretation is that differences in local coordination occur among the REE ions in calcite; the REE impurities Dy and $\mathrm{Yb}$ are dominantly in six-fold coordination and the REEs Nd and Sm are dominantly in seven-fold coordination, the latter being a modified Ca site. 


\section{References}

D'Angelo, P., N. V. Pavel, and M. Borowski. J. Synchrotron Rad. 8, 666-668 (2001). Shannon R. D., Acta Cryst. A32, 751-767 (1976). Solera J. A., J. Garcia, and M. G. Proietti. Phys. Rev. B 51, 2678-2686 (1995).

Zhong S. and A. Mucci. Geochim. Cosmochim. Acta 59, 443-453 (1995).

\section{Preparation of $\mathrm{Pt} / \mathrm{TiO}_{2}$} Nanocomposite Thin Films by Pulsed Laser Deposition and Their Photoelectrochemical

\section{Behaviors}

T. Sasaki, ${ }^{(a)}$ N. Koshizaki, ${ }^{(a)}$

J. W. Yoon, ${ }^{(a)}$ and K. M. Beck ${ }^{(b)}$

This study was supported by the Center of Excellence (COE) project from the Science and Technology Agency (STA) of Japan. Dr. Beck was supported by Japan STA and COE research fellowships, and the Office of Basic Energy Sciences, U.S. Department of Energy.

(a) Nanoarchitectonics Research Center, National Institute of Advanced Industrial Science and Technology, Japan.

(b) Pacific Northwest National Laboratory.

The size reduction of materials to the nanometer scale reveals unique physical properties (Chiang 1997). To use these properties, we must develop the capability to build up tailored nanostructures for a given function by controlling the materials at the molecular level (Roco 1999; Hu and Shaw 1999). By preparing nanocomposites made of oxide films containing nano-sized semiconductor or metal particles, we can create systems with unusual optical and electrical properties. Such unique optical and chemical properties result from quantum size effects of the nanoparticles embedded in the matrix and from phenomena occurring at the interface between nanoparticles and matrix (Sasaki et al. 1998).

$\mathrm{TiO}_{2}$ in its anatase form is far less understood than the rutile modification. The rutile form is stable at high temperatures $\left(>700^{\circ} \mathrm{C}\right)$ and is usually obtained when pure $\mathrm{TiO}_{2}$ crystal is synthesized. Recent application of colloidal anatase in novel photochemical solar cells, high mobility n-type charge carriers, and the metal-nonmetal transition in the impurity band of reduced anatase thin films have stimulated interest and investigations (Mo and Ching 1995; Durand et al. 1995). Titanium dioxide is a promising photoactive material, though it has a bandgap of $3.2 \mathrm{eV}$ that results in almost no functionality in the visible light range. The preparation of nanocomposites dispersed with noble metal particles is an approach to overcome such a drawback. Zhao et al. (1996) reported that the $\mathrm{TiO}_{2}$ films containing $\mathrm{Au}$ and $\mathrm{Ag}$ metal particles also have photosensitivity to visible light, where the anodic photocurrent for oxygen evolution reaction in aqueous solutions can be observed at the wavelengths shorter than $700 \mathrm{~nm}$. Pt nanoparticles are an effective sensitizer of $\mathrm{TiO}_{2}$ photocatalysts because of their high catalytic activity and low over-potential for hydrogen evolution reaction. In the decomposition of water in the $\mathrm{Pt} / \mathrm{TiO}_{2}$ system, $\mathrm{Pt}$ nanoparticles on $\mathrm{TiO}_{2}$ can effectively trap the photoexcited electrons in the conduction band of $\mathrm{TiO}_{2}$, followed by the evolution of hydrogen in aqueous solutions. $\mathrm{Pt} / \mathrm{TiO}_{2}$ composites and nanocomposites have been prepared by a variety of methods such as sol-gel, sputtering, pulsed laser deposition and thermal decomposition methods. Choi et al. (1992) investigated the photoelectrochemical properties of $\mathrm{Pt} / \mathrm{TiO}_{2}$ electrodes prepared by thermal oxidation of titanium sheets followed by the electrodeposition of platinum, where anodic photocurrents were observed only at wavelengths of 200$400 \mathrm{~nm}$. This result was quite different from the $\mathrm{Au} / \mathrm{Ti} \mathrm{O}_{2}$ and $\mathrm{Ag} / \mathrm{TiO}_{2}$ systems described above. We have previously reported the preparation of $\mathrm{Pt} / \mathrm{TiO}_{2}$ nanocomposite thin films by pulsed laser deposition (Beck et al. 1999). In this paper we demonstrate the photoelectrochemical properties of these thin films with well-controlled nanostructures in UV-visual light range. The mechanism of the photo-response in the visible light of the $\mathrm{Pt} / \mathrm{TiO}_{2}$ nanocomposite thin film electrodes is also discussed.

We have previously described the synthesis of $\mathrm{Pt} / \mathrm{TiO}_{2}$ nanocomposite films deposited on quartz slides (Beck et al. 1999). Here we use the same process, but deposit the thin films on indium tin oxide (ITO) glass substrates for photo-electrochemical measurements by pulsed laser deposition. The mixed pellets of $\mathrm{Pt}$ and the $\mathrm{TiO}_{2}$ were used as the target materials, and the simultaneous ablation of both $\mathrm{Pt}$ and $\mathrm{TiO}_{2}$ components was used to deposit the $\mathrm{Pt} / \mathrm{TiO}_{2}$ nanocomposite films. 
All the samples were heated in air at $600^{\circ} \mathrm{C}$ for crystallization, since the as-deposited films prepared at room temperature were always amorphous. The structures of the films were examined by X-ray diffraction (XRD). Nano-scale inhomogeneity and microstructure of the films were analyzed by transmission electron microscope (TEM) observation. The chemical state of $\mathrm{Pt}$, and the $\mathrm{Pt} / \mathrm{Ti}$ atomic ratio in the nanocomposites were examined by X-ray photoelectron spectroscopy (XPS). The optical transmittance of the nanocomposite films was measured using a UVVis spectrometer. Luminescence emission was also measured using a laser-based spectrometer with a cryostat at $24 \mathrm{~K}$ in vacuum with an excitation of $325 \mathrm{~nm}$ light.

The annealed and as-deposited $\mathrm{Pt} / \mathrm{TiO}_{2}$ nanocomposite films on ITO glass substrate were used for photoelectrochemical measurement. The electrical lead wire was attached to the ITO surface by silver paste. The substrates were covered with epoxy resin, except for the nanocomposite and $\mathrm{TiO}_{2}$ surfaces. A conventional three-electrode system was used in the photoelectrochemical experiments; nanocomposite samples, Pt and $\mathrm{Ag} / \mathrm{AgCl}$ electrodes were used as working, counter, and reference electrodes, respectively. The potential of the working electrode in the electrolyte $(0.1 \mathrm{M}$ $\mathrm{Na}_{2} \mathrm{SO}_{4}$ aqueous solution) was controlled using a potentiostat. Photocurrents were measured under the irradiation of the light through a monochromator and an electronic shutter.

Figure 3.23 displays the XPS spectra of Pt $4 \mathrm{f}$ levels in the as-deposited film deposited from 20 percent $\mathrm{Pt}$ by weight target at $5.7 \mathrm{~J} / \mathrm{cm}^{2}$ target. The $\mathrm{Pt} / \mathrm{Ti}$ atomic ratios in the deposited films are more strongly dependent on the initial content of Pt in the target than on laser fluence. Pt/Ti atomic ratios in the deposited films increased with the platinum content of the targets. The atomic ratios in deposited films were always smaller than those in the target materials. This result could reflect the difference in the ablation threshold of platinum and $\mathrm{TiO}_{2} . \mathrm{TiO}_{2}$ is more effectively ablated by the laser irradiation than platinum because platinum has a higher ablation threshold than $\mathrm{TiO}_{2}$ (Chrisey and Haber 1994). In contrast, the film thickness is more strongly dependent on the laser fluence than on the initial Pt content in the target. Film thickness increased only with an increase in the laser fluence under the deposition. Higher fluence can

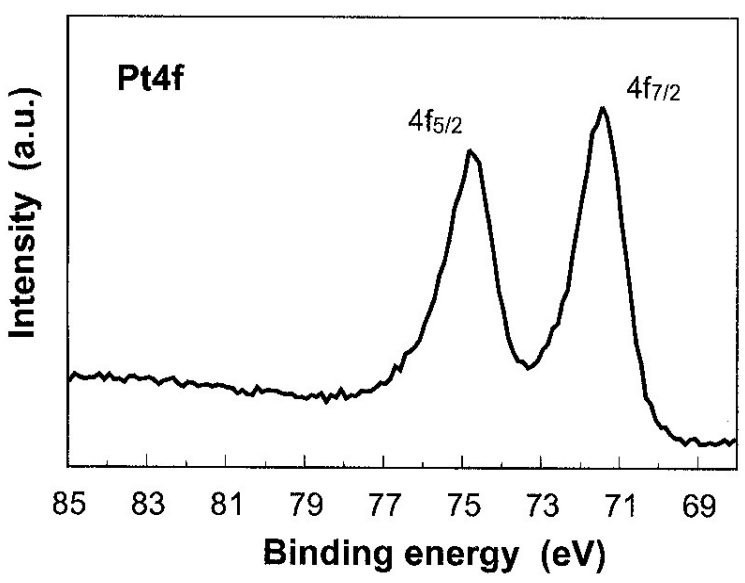

Figure 3.23. XPS spectra of Pt $4 \mathrm{f}$ levels in the asdeposited film deposited from the $20 \%$ Pt by weight target at $5.7 \mathrm{~J} / \mathrm{cm}^{2}$.

increase the flux of evaporated species from the target materials, resulting in the thicker deposits. A binding energy of around $71.4 \mathrm{eV}$ was observed in all of the as-deposited $\mathrm{Pt} / \mathrm{TiO}_{2}$ nanocomposite films, where the $\mathrm{Pt} / \mathrm{Ti}$ atomic ratio was less than 0.06 , indicating that $\mathrm{Pt}$ in the nanocomposite films is a metallic state (Davidson et al. 1987). Platinum was frequently oxidized to $\mathrm{PtO}$ or $\mathrm{PtO}_{2}$ during the deposition when $\mathrm{Pt} / \mathrm{TiO}_{2}$ nanocomposite films were deposited by cosputtering (Mo and Ching 1995). Post-annealing of sputtered films was sometimes required to form metallic Pt nanoparticles in such co-sputtered films. For pulsed laser deposition, metallic Pt nanoparticles can easily be obtained in $\mathrm{Pt} / \mathrm{TiO}_{2}$ nanocomposite films without the post-annealing. Nanoparticles with diameters of about $30 \mathrm{~nm}$ are observed in TEM (Beck et al. 1999). Single electron diffraction was observed in a few, and the cubic fringe spacings were estimated to be 2.27 and $2.00-1.92 \AA$. These values agree well with Pt metallic lattices ( $\left(\begin{array}{lll}1 & 1 & 1\end{array}\right)$ and (2 00$)$, respectively (CAS \#7440-06-04, =2 $265 \AA$ as prepared at NBS, Gaitherburg, MD).

In order to estimate the optical bandgap of the films, the UV-Vis spectra of samples were recorded after annealing. The variation in the absorption coefficient with photon energy for direct allowed band-to-band transition can be represented as, $\mathrm{a}^{2}=\mathrm{a}_{0}^{2} \mathrm{~h} v(\Delta \mathrm{E})$, where $a$ is the absorption coefficient and $\Delta \mathrm{E}$ is the bandgap. After correcting for reflection losses, the optical bandgap can be estimated by extrapolating the linear portion of the curve to a $2=0$. For the pure $\mathrm{TiO}_{2}$ film, we obtain a value of $\Delta \mathrm{E}=3.15 \mathrm{eV}$. The 
bandgaps of $\mathrm{Pt} / \mathrm{TiO}_{2}$ nanocomposite films prepared from 5, 10 and 20 percent Pt by weight targets were estimated to be 2.9, 2.8 and $2.3 \mathrm{eV}$, respectively (Beck et al. 1999). In addition, luminescence emission was observed by UV irradiation at $680-800 \mathrm{~nm}$ only from $\mathrm{Pt} / \mathrm{TiO}_{2}$. These results suggest that the some energy levels could be formed in the bandgap of $\mathrm{TiO}_{2}$.

We expected that photoelectrochemical properties of $\mathrm{Pt} / \mathrm{TiO}_{2}$ nanocomposite electrodes would be completely different from those of pure $\mathrm{TiO}_{2}$ electrodes, and that the former should have a photo-response in the visible light range. Indeed, the photoelectrochemical properties of $\mathrm{Pt} / \mathrm{TiO}_{2}$ nanocomposite electrodes were completely different from those of $\mathrm{TiO}_{2}$ electrodes. Figure 3.24 illustrates a typical cyclic voltammogram of the heated $\mathrm{Pt} / \mathrm{TiO}_{2}$ nanocomposite electrode prepared from 5 percent Pt by weight target at $5.7 \mathrm{~J} / \mathrm{cm}^{2}$. The heated and as-deposited $\mathrm{Pt} / \mathrm{TiO}_{2}$ nanocomposite electrodes have almost the same behavior. Under anodic polarization with the irradiation of the total light from a Xe lamp, anodic photocurrents for the oxygen evolution reaction were observed at potentials above $0.2 \mathrm{~V}$. The anodic photocurrents of all the nanocomposite electrodes were smaller than those of pure $\mathrm{TiO}_{2}$ electrodes. Cathodic photocurrents were also observed in all nanocomposite electrodes. These photocurrents increased in the solution under oxygen bubbling rather than nitrogen bubbling, indicating that the

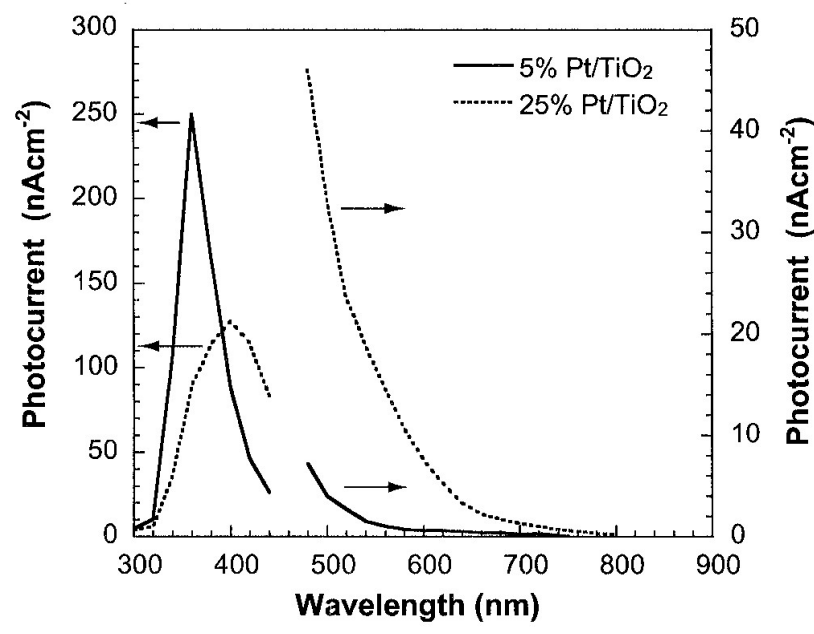

Figure 3.24. A typical cyclic voltammogram of the heated $\mathrm{Pt} / \mathrm{TiO}_{2}$ nanocomposite electrode prepared from $5 \% \mathrm{Pt}$ by weight target at $5.7 \mathrm{~J} / \mathrm{cm}^{2}$. cathodic photocurrent can be ascribed to the reduction of oxygen by photoexcited electrons.

Figure 3.25 shows the anodic photocurrent at $1.0 \mathrm{~V}$ for $\mathrm{Pt} / \mathrm{TiO}_{2}$ nanocomposite electrodes prepared from 5 and 20 percent Pt by weight target as a function of the irradiating light wavelength. The photocurrents of as-deposited nanocomposite electrodes were higher than those of heated electrodes.

This trend was the same as the cyclic voltammogram of all samples. Many defects in as-deposited samples could affect the electrical conductivities, resulting in the higher photocurrent. However, the anodic photocurrent of as-deposited electrodes was a little unstable. In contrast, anodic photocurrents in the wavelength region from 450 to $750 \mathrm{~nm}$ were clearly observed and stable in the annealed $\mathrm{Pt} / \mathrm{TiO}_{2}$ nanocomposite electrodes prepared from 20 percent Pt by weight target. The anodic photocurrent in visible light is higher in the $\mathrm{Pt} / \mathrm{TiO}_{2}$ nanocomposite film prepared from 20 percent than 5 percent $\mathrm{Pt}$ by weight target. Metallic $\mathrm{Pt}$ nanoparticles exist in the heated $\mathrm{Pt} / \mathrm{TiO}_{2}$ nanocomposite films prepared from 20 percent $\mathrm{Pt}$ by weight target, as previously stated. These results suggest that the metallic Pt nanoparticles could play an important role in creating energy levels in the

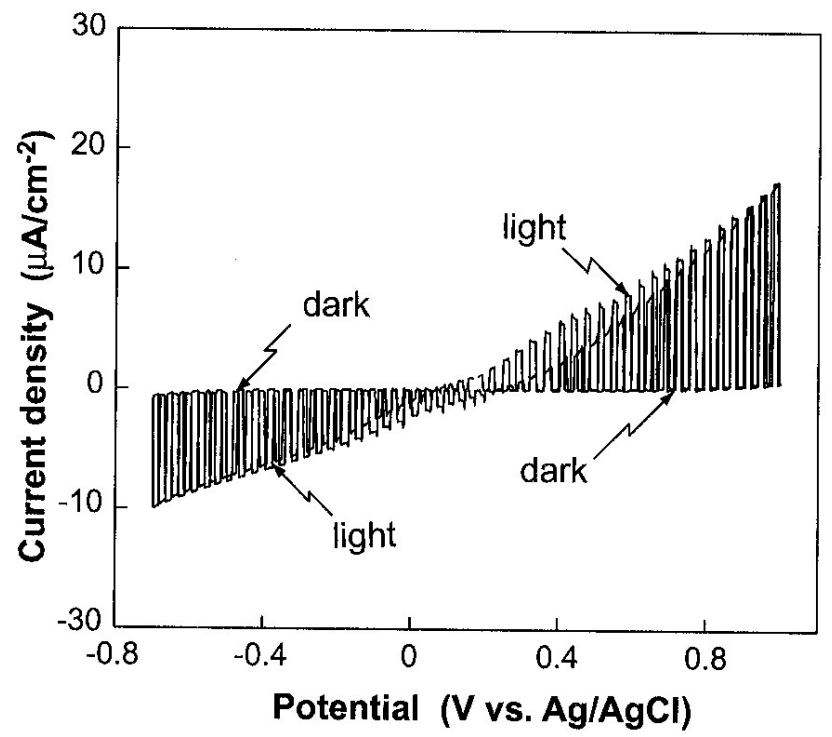

Figure 3.25. The anodic photocurrent at $1.0 \mathrm{~V}$ for $\mathrm{Pt} / \mathrm{TiO}_{2}$ nanocomposite electrodes prepared from 5 and 20 percent $\mathrm{Pt}$ by weight target as a function of the irradiating light wavelength. 
bandgap. It is well known that a Schottky barrier is formed at the interface between a metal and semiconductor. The barrier height of the $\mathrm{Pt}-\mathrm{TiO}_{2}$ interface is estimated to be $1.64 \mathrm{eV}$ from the work function of $\mathrm{Pt}$ $(5.64 \mathrm{eV})$ (Michaelson 1997) and the electron affinity of $\mathrm{TiO}_{2}(4.0 \mathrm{eV})$ (Choi et al. 1992). The photoexcitation of electrons from the Pt metal to the conduction band of $\mathrm{TiO} 2$ could take place under the irradiation of light with energy exceeding $1.64 \mathrm{eV}$.

It should be noted that the Pt nanoparticles are isolated in $\mathrm{Pt} / \mathrm{TiO}_{2}$ nanocomposite films, indicating that each Schottky barrier between $\mathrm{Pt}$ nanoparticles and $\mathrm{TiO}_{2}$ is localized in the $\mathrm{TiO}_{2}$ matrix. The number density of such localized interfaces can also be important for the total photoresponse of $\mathrm{Pt} / \mathrm{TiO}_{2}$ nanocomposite films. A high number density of $\mathrm{Pt}$ nanoparticles in $\mathrm{TiO}_{2}$ allows production of an efficient electronic state density of the energy levels, which can contribute to the photoexcitation with lower energy than that of the bandgap of $\mathrm{TiO}_{2}$, resulting in a unique photoresponse in visible light.

$\mathrm{Pt} / \mathrm{TiO}_{2}$ nanocomposite films were prepared from mixture targets of $\mathrm{Pt}$ and $\mathrm{TiO}_{2}$ by pulsed laser deposition. The $\mathrm{Pt} / \mathrm{Ti}$ atomic ratio in films was controlled by the initial content of Pt in the target, and was independent of laser fluence. It is inferred from TEM, XPS and XRD measurements that as-deposited $\mathrm{Pt} / \mathrm{TiO}_{2}$ nanocomposite films are composed of metallic Pt nanoparticles with diameters of about $30 \mathrm{~nm}$ and an amorphous $\mathrm{TiO}_{2}$ matrix, which are crystallized into the dominant crystal structure of anatase phase after heating at $600^{\circ} \mathrm{C}$. The optical bandgap of $\mathrm{Pt} / \mathrm{TiO}_{2}$ nanocomposite films was less than $2.3 \mathrm{eV}$, and a photoluminescence emission between 680 and $800 \mathrm{~nm}$ at $24 \mathrm{~K}$ was observed. The anodic photocurrents of $\mathrm{Pt} / \mathrm{TiO}_{2}$ nanocomposite electrodes at $1.0 \mathrm{~V}$ were also observed in visible light. Some energy levels can be formed by the interface between Pt nanoparticles and $\mathrm{TiO}_{2}$, which also affect both the photoelectrochemical and optical properties of $\mathrm{Pt} / \mathrm{TiO}_{2}$ nanocomposite films. The localized Schottky barrier at the interface between $\mathrm{Pt}$ nanoparticles and $\mathrm{TiO}_{2}$ could contribute to the photoresponse in the visible light range.

\section{References}

Beck, ,K. M., T. Sasaki, and N. Koshizaki, Chem. Phys. Letts 301, 336 (1999).

Chiang, Y. M., J. Electroceram 1, 205 (1997).

Choi,Y. K., S. S. Seo, K. H. Chjo, Q. W. Choi, and S. M. Park, J. Electrochem. Soc. 139, 1803 (1992).

Chrisey, D. B., and G. K. Huber (Eds.), Pulsed Laser Deposition of Thin Films, Wiley, New York, 455 (1994).

Davidson, M., G. Hofiund, L. Niinisto, and H. Laitinen, J. Electroanal. Chem. 228, 471 (1987).

Durand, H.-A., J.-H. Brimaud, O. Hellman, H. Shibata, S. Sakuragi, Y. Makita, D. Gesbert, and P. Meyrues, Appl. Surf. Sci. 86, 122 (1995).

Hu, E. L., and D. T. Shaw, in: R. W. Siegel, E. L. Hu, and M. C. Roco (Eds.), Nanostructure Science and Technology, Kluwer Academic Publishers, Dordrecht, 15-33 (1999).

Michaelson H. B., J. Appl. Phys. 48, 4729 (1977).

Mo, S.-D., and W. Y. Ching, Phys. Rev. B 51, 13023 (1995).

Roco, M. C., J. Nanoparticle Res. 1, 1 (1999).

Sasaki, T., N. Koshizaki, S. Terauchi, H. Umehara, Y. Matsumoto, and M. Koinuma, Nanostruct. Mater. 8, 1077 (1998).

Zhao, G., H. Kozuka, and T. Yoko, Thin Solid Films 277147 (1996). 


\section{Cluster Models of the Condensed Phase}

This research is aimed at providing a molecular-level understanding of solvation and subsequent reactions in simple and complex systems as they relate to the chemistry of complex wastes, contaminated solids and groundwater, and other systems found in nature. A major experimental and theoretical effort is devoted to understanding surface and interface properties using cluster models to study structure and bonding. Small and controllable cluster systems provide atomic-level models that enable us to understand bulk surfaces and defect sites. They are also an excellent testing ground to benchmark theories intended for large and "real-world" systems. 


\section{Clusters and Multiply Charged} Anions: Model Studies and Solvations

\author{
L. S. Wang, X. B. Wang, ${ }^{(a)}$ \\ X. Yang ${ }^{(a)}, J$. B. Nicholas, ${ }^{(b)}$ and \\ E. R. Vorpagel ${ }^{(c)}$
}

Supported by DOE Office of Basic Energy Sciences.

(a) Washington State University.

(b) Genentech, Inc.

(c) Pacific Northwest National Laboratory, William R. Wiley Environmental Molecular Sciences Laboratory.

\section{Photodetachment of $\mathrm{F}^{-}\left(\mathrm{H}_{2} \mathrm{O}\right)_{\mathrm{n}}(n=1$ to 4$)$}

\section{(Yang et al. 2001)}

We carried out a photoelectron spectroscopic study of $\mathrm{F}^{-}\left(\mathrm{H}_{2} \mathrm{O}\right)_{\mathrm{n}}(n=1-4)$ at 193 and $157 \mathrm{~nm}$. In addition to a detachment feature from $\mathrm{F}^{-}$, we observed a higher binding energy feature in all spectra due to ionization of the solvent molecules leading to $\mathrm{F}^{-}\left(\mathrm{H}_{2} \mathrm{O}\right)_{n}{ }^{+}$chargetransfer (CT) states (Figure 4.1). Detachment of an electron from $\mathrm{F}^{-}$in $\mathrm{F}^{-}\left(\mathrm{H}_{2} \mathrm{O}\right)$ reaches the transition state region of the reaction, $\mathrm{F}+\mathrm{H}_{2} \mathrm{O} \rightarrow \mathrm{HF}+\mathrm{OH}$. The bound to non-bound transition resulted in a long tail at the low binding energy side in the photoelectron spectrum of $\mathrm{F}^{-}\left(\mathrm{H}_{2} \mathrm{O}\right)$. We estimated that the neutral $\mathrm{F} \cdots \mathrm{H}_{2} \mathrm{O}$ complex at the anion geometry, which is near the transition state region of the $\mathrm{H}$-abstraction reaction, is $6 \pm 1 \mathrm{kcal} / \mathrm{mol}$ above the $\mathrm{F}+\mathrm{H}_{2} \mathrm{O}$ asymptote, consistent with a previous estimation of a $4 \mathrm{kcal} / \mathrm{mol}$ entrance barrier height for this reaction.

\section{Bulk-Like Features in the Photoemission Spectra of Hydrated Doubly-Charged Anion Clusters}

\section{(Wang et al. 2001a)}

We produced gaseous hydrated clusters of sulfate and oxalate anions $\left[\mathrm{SO}_{4}{ }^{2-}\left(\mathrm{H}_{2} \mathrm{O}\right)_{\mathrm{n}}\right.$ and $\mathrm{C}_{2} \mathrm{O}_{4}{ }^{2-}\left(\mathrm{H}_{2} \mathrm{O}\right)_{\mathrm{n}}$ $(n=4-40)$ ] (Figure 4.2). Photoelectron spectra of these clusters revealed that the solute dianions were in the center of the water cluster, $\left(\mathrm{H}_{2} \mathrm{O}\right)_{\mathrm{n}}$. For small clusters, the spectra were characteristic of the

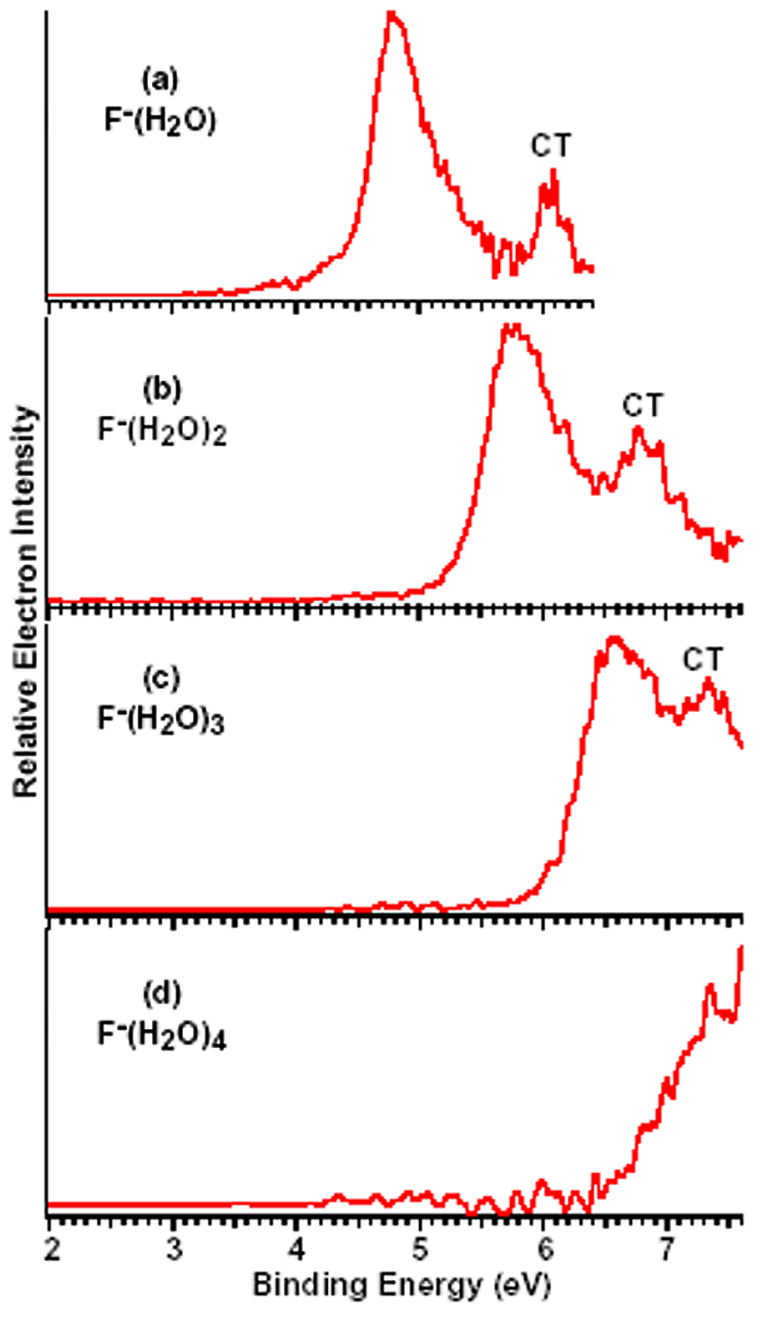

Figure 4.1. Photoelectron spectra of $\mathrm{F}^{-}\left(\mathrm{H}_{2} \mathrm{O}\right)_{\mathrm{n}}$ $(n=1-4)$ at $157 \mathrm{~nm}$.

respective solutes, but beyond the first solvation shell $(n \approx 12)$, features in the spectra from the solutes were diminished and a new feature from ionization of water emerged, analogous to bulk aqueous solutions. For large clusters with dimensions over one nanometer, the solute photoemission features disappeared and the spectra were dominated by the ionization of water as the solvent coverage increased. A smooth transition from gas-phase clusters to behavior of electrolyte solutions was clearly revealed. The large solvated clusters can be used as molecular models to investigate the photophysics and chemistry of aqueous electrolyte solutions. 

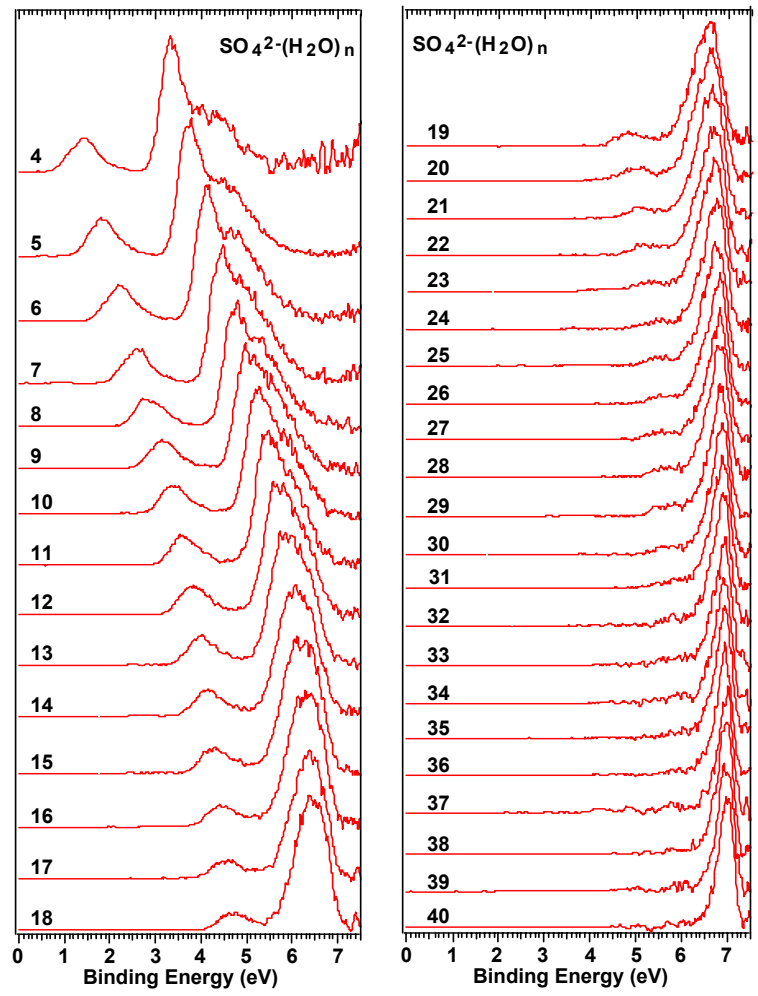

Figure 4.2. Photoelectron spectra of $\mathrm{SO}_{4}{ }^{2-}\left(\mathrm{H}_{2} \mathrm{O}\right)_{\mathrm{n}}$ $(n=4-40)$ at $157 \mathrm{~nm}(7.866 \mathrm{eV})$.

\section{Experimental and Theoretical Investigations of the Stability, Energetics, and Structures of $\mathrm{H}_{2} \mathrm{PO}_{4}{ }^{-}$, $\mathrm{H}_{2} \mathrm{P}_{2} \mathrm{O}_{7}{ }^{2-}$, and $\mathrm{H}_{3} \mathrm{P}_{3} \mathrm{O}_{10}{ }^{2-}$ in the Gas Phase}

\section{(Wang et al., 2001b)}

The stability, energetics, and structures of three common inorganic phosphate species, $\mathrm{H}_{2} \mathrm{PO}_{4}{ }_{4}$, $\mathrm{H}_{2} \mathrm{P}_{2} \mathrm{O}_{7}{ }^{2-}, \mathrm{H}_{3} \mathrm{P}_{3} \mathrm{O}_{10}{ }^{2-}$, and their corresponding neutral radical and monoanions, were investigated in the gas phase using photodetachment photoelectron spectroscopy and theoretical calculations. We found that $\mathrm{H}_{2} \mathrm{P}_{2} \mathrm{O}_{7}{ }^{2-}$ and $\mathrm{H}_{3} \mathrm{P}_{3} \mathrm{O}_{10}{ }^{2-}$ are stable in the gas phase with adiabatic electron binding energies of 1.16 and $2.45 \mathrm{eV}$, respectively. A very high adiabatic electron binding energy of $4.57 \mathrm{eV}$ was measured for $\mathrm{H}_{2} \mathrm{PO}_{4}{ }^{-}$. The intramolecular Coulomb repulsion energies in $\mathrm{H}_{2} \mathrm{P}_{2} \mathrm{O}_{7}{ }^{2-}(\sim 2.7 \mathrm{eV})$ and $\mathrm{H}_{3} \mathrm{P}_{3} \mathrm{O}_{10}{ }^{2-}(\sim 2.3 \mathrm{eV})$ were estimated from photon-energy-dependent photoelectron spectra. Density-functional theory calculations were used to search the optimal geometries for both the doubly and singly charged species. We found only one minimum energy conformation for $\mathrm{H}_{2} \mathrm{P}_{2} \mathrm{O}_{7}{ }^{2-}$ with two intramolecular $\mathrm{H}$-bonds and $\mathrm{C}_{2}$ symmetry, and three minimum energy structures for $\mathrm{H}_{3} \mathrm{P}_{3} \mathrm{O}_{10}{ }^{2-}$. The lowest energy structure of $\mathrm{H}_{3} \mathrm{P}_{3} \mathrm{O}_{10}{ }^{2-}$ has three intramolecular $\mathrm{H}$-bonds that do not share a common oxygen atom. The calculated electron detachment energy of $\mathrm{H}_{2} \mathrm{PO}_{4}{ }^{-}$agrees well with the experimental value, but the calculated detachment energies for $\mathrm{H}_{2} \mathrm{P}_{2} \mathrm{O}_{7}{ }^{2-}$ and $\mathrm{H}_{3} \mathrm{P}_{3} \mathrm{O}_{10}{ }^{2-}$ are $\sim 0.3$ and $\sim 0.7 \mathrm{eV}$ smaller than the experimental values, respectively. The observed spectral features, due to removal of electrons from lone-pair oxygen orbitals in the phosphate groups (Figure 4.3), were assigned qualitatively on the basis of the theoretical calculations.

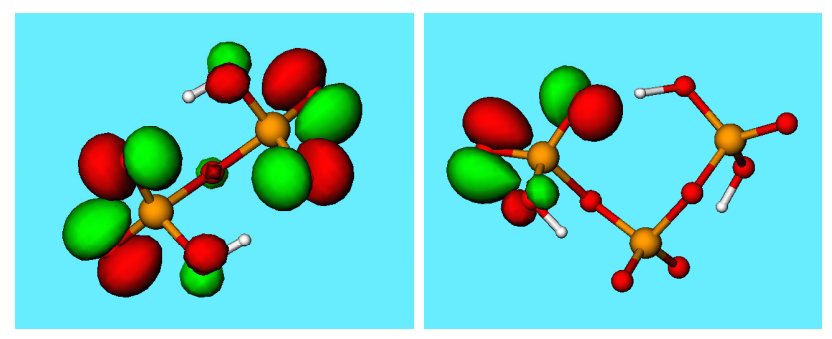

Figure 4.3. The highest occupied molecular orbitals for (a) $\mathrm{H}_{2} \mathrm{P}_{2} \mathrm{O}_{7}{ }^{2-}$ and (b) $\mathrm{H}_{3} \mathrm{P}_{3} \mathrm{O}_{10}{ }^{2-}$.

\section{References}

Wang, X. B., X. Yang, J. B. Nicholas, and

L. S. Wang, Science 294, 1322-1325 (2001a).

Wang, X. B., E. R. Vorpagel, X. Yang, and

L. S. Wang, J. Phys. Chem. A 105, 10468-10474 (2001b).

Yang, X., X. B. Wang, and L. S. Wang, J. Chem. Phys. 115, 2889-2892 (2001). 


\section{Probing the Electronic} Structure of Transition Metal Clusters and Complexes

\author{
L. S. Wang, X. B. Wang, ${ }^{(a)}$ \\ H. J. Zhai, ${ }^{(a)}$ S. Liu, ${ }^{(a)}$ R. Brown, ${ }^{(b)}$ \\ P. Schwerdtfeger, ${ }^{(b)}$ D. Schroder, ${ }^{(c)}$ \\ H. Schwarz, ${ }^{(c)}$ G. L. Gutsev, ${ }^{(d)}$ and \\ P. Jena ${ }^{(d)}$
}

Supported by National Science Foundation (CHEM).

(a) Washington State University.

(b) University of Auckland, New Zealand.

(c) Technical University of Berlin, Germany.

(d) Virginia Commonwealth University.

\section{The Electronic Structure of $\mathrm{CuCl}_{2}$ and $\mathrm{CuBr}_{2}$ from Anion Photoelectron Spectroscopy and $A b$ Initio Calculations}

\section{(Wang et al. 2001)}

The electronic structures of $\mathrm{CuX}_{2}(\mathrm{X}=\mathrm{Cl}$ and $\mathrm{Br})$ were investigated in the gas phase by means of anion photodetachment photoelectron spectroscopy and $a b$ initio theory. The photoelectron spectra of $\mathrm{CuX}_{2}^{-}$ were recorded at two photon energies, 193 and $157 \mathrm{~nm}$. Well-resolved and rich photodetachment features in the spectra provide unprecedented details for the low-lying electronic states of $\mathrm{CuCl}_{2}$ and $\mathrm{CuBr}_{2}$. The excitation energies for five low-lying electronic states of $\mathrm{CuX}_{2}$ were determined, and they explain well the two previously observed optical absorption bands. The electron affinities for $\mathrm{CuCl}_{2}$ and $\mathrm{CuBr}_{2}$ were determined to be identical, $4.35 \pm 0.05 \mathrm{eV}$ within the experimental uncertainty. Both the anions and neutral $\mathrm{CuX}_{2}$ species were calculated to be linear with only a slight bond length variation between the charged and neutral species. The calculated electron affinities and vertical excitation energies for the excited states agree well with the experimental values, yielding a definite assignment for the electronic states of $\mathrm{CuCl}_{2}$ and $\mathrm{CuBr}_{2}$.

\section{Electronic and Structural Evolution of Co $_{n}$ Clusters $(n=1-108)$ by Photoelectron Spectroscopy}

\section{(Liu et al. 2001)}

Well-resolved photoelectron spectra were obtained for $\mathrm{Co}_{\mathrm{n}}{ }^{-}(n=1-108)$ clusters. The data provide evidence for structural transitions and reveal in detail the molecular to bulk electronic evolution in Co clusters. Clusters with $n<20$ behave molecular-like with discrete transitions and dramatic size variations. Clusters with $n \geq 20$ behave bulk-like with broad spectral features and smooth size evolution. But discrete and well-resolved spectral features are observed around $n=55$, evident of a highly symmetric $\mathrm{Co}_{55}$ cluster. The molecular to bulk-like transition is also shown in the electron affinities as a function of size, which obey the metallic droplet model above $\mathrm{Co}_{20}$ and extrapolate to the bulk workfunction.

\section{Electronic Structure of Chromium Oxides, $\mathrm{CrO}_{n}{ }^{-}$and $\mathrm{CrO}_{n}(\mathrm{n}=1-5)$ from Photoelectron Spectroscopy and Density Functional Theory Calculations}

\section{(Gutsev et al. 2001)}

The electronic structure of $\mathrm{CrO}_{\mathrm{n}}{ }^{-}$and $\mathrm{CrO}_{\mathrm{n}}(n=1-5)$ was investigated using anion photoelectron spectroscopy and density functional theory. Photoelectron spectra of $\mathrm{CrO}_{\mathrm{n}}{ }^{-}$were obtained at several photon energies and yielded electron affinities, vibrational and electronic structure information about the neutral $\mathrm{CrO}_{\mathrm{n}}$ species. Density functional theory calculations were carried out for both the neutrals and anions (Figure 4.4) and were used to interpret the

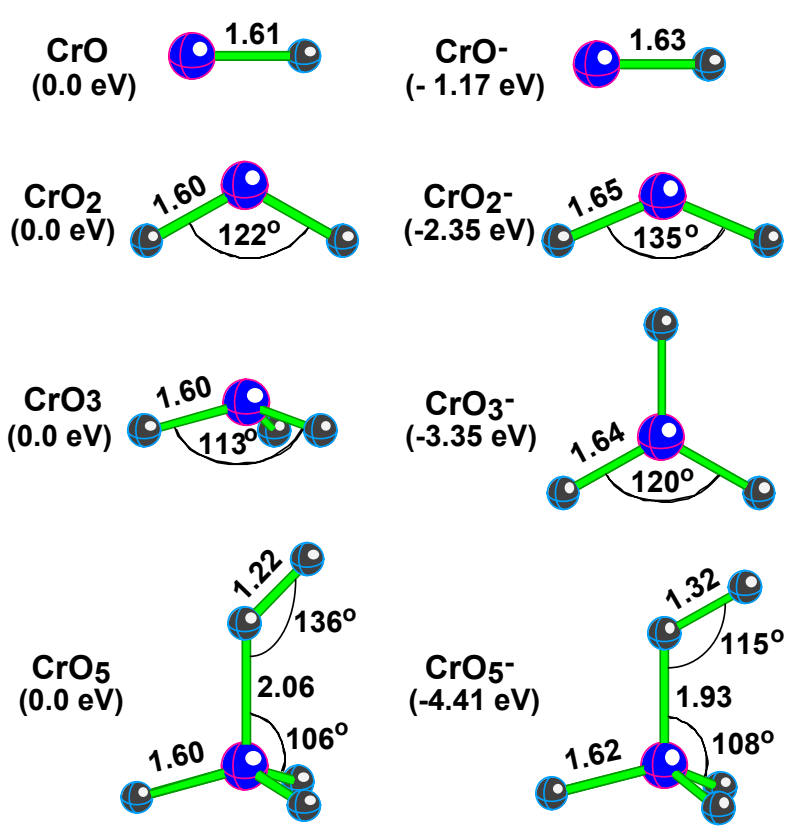

Figure 4.4. Ground state geometrical configurations of $\mathrm{CrO}, \mathrm{CrO}_{2}, \mathrm{CrO}_{3}$, and $\mathrm{CrO}_{5}$ and their anions. 
experimental spectra. Several low-lying electronic states of $\mathrm{CrO}$ were observed and assigned from photodetachment of the $\mathrm{CrO}^{-}$ground state $\left({ }^{6} \Sigma^{+}\right)$and an excited state $\left({ }^{4} \Pi\right)$, which is only $0.1 \mathrm{eV}$ higher. The main spectral features of $\mathrm{CrO}_{2}^{-}$were interpreted based on a $\mathrm{C}_{2 \mathrm{v}} \mathrm{CrO}_{2}^{-}\left({ }^{4} \mathrm{~B}_{1}\right)$. A very weak $\mathrm{Cr}\left(\mathrm{O}_{2}\right)^{-}$isomer was also observed with lower electron binding energies. Relatively simple and vibrationally resolved spectra were observed for $\mathrm{CrO}_{3}{ }^{-}$, which was determined to be $\mathrm{D}_{3 \mathrm{~h}}$. The $\mathrm{CrO}_{3}$ neutral was calculated to be $\mathrm{C}_{3 \mathrm{v}}$ with the $\mathrm{Cr}$ atom slightly out of the plane of the three $\mathrm{O}$ atoms. The spectrum of $\mathrm{CrO}_{4}{ }^{-}$revealed a very high electron binding energy. Several isomers of $\mathrm{CrO}_{4}^{-}$ were predicted and the ground state has a distorted tetrahedral structure $\left(\mathrm{C}_{2}\right)$ without any $\mathrm{O}-\mathrm{O}$ bonding. Only one stable structure was predicted for $\mathrm{CrO}_{5}{ }^{-}$with a superoxo $\mathrm{O}_{2}$ bonded to a $\mathrm{C}_{3 \mathrm{v}} \mathrm{CrO}_{3}$.

\section{References}

Gutsev, G. L., P. Jena, H. J. Zhai, and L. S. Wang, J.Chem. Phys. 115, 7935-7944 (2001).

Liu, S. R., H. J. Zhai, and L. S. Wang, Phys. Rev. B. 64, 153402 (2001).

Wang, X. B., L. S. Wang, R. Brown,

P. Schwerdtfeger, D. Schröder, and H. Schwarz,

J. Chem. Phys. 114, 7388-7395 (2001).

\section{Investigation of Metal-Carbon Mixed Clusters and Aluminum Alloy Clusters}

\section{S. Wang, X. Li, ${ }^{(a)}$ H. F. Zhang, ${ }^{(a)}$ H. J. Zhai, ${ }^{(a)}$ A. I. Boldyrev, ${ }^{(b)}$ and J. D. Corbett ${ }^{(c)}$}

Supported by National Science Foundation (DMR).

(a) Washington State University.

(b) Utah State University.

(c) Iowa State University.

\section{Observation of All-Metal Aromatic Molecules}

\section{(Li et al. 2001a)}

Aromaticity is a concept invented to account for the unusual stability of an important class of organic molecules, the aromatic compounds. Here we report experimental and theoretical evidence of aromaticity in all-metal systems. A series of bimetallic clusters $\mathrm{MAl}_{4}{ }^{-}(\mathrm{M}=\mathrm{Li}, \mathrm{Na}, \mathrm{Cu})$ were created and studied using photoelectron spectroscopy and ab initio calculations. All the $\mathrm{MAl}_{4}{ }^{-}$species possess a pyramidal ground state structure containing an $\mathrm{M}^{+}$ cation interacting with a square $\mathrm{Al}_{4}{ }^{2-}$ unit and a lowlying isomer in planar geometries (Figure 4.5). $A b$ initio studies indicate that $\mathrm{Al}_{4}^{2-}$ exhibits characteristics of aromaticity with two delocalized $\pi$-electrons (thus following the $4 n+2$ electron counting rule) and a square-planar structure and maintains its structural and electronic features in all the $\mathrm{MAl}_{4}{ }^{-}$complexes. The current findings expand the aromaticity concept into the arena of all-metal species.
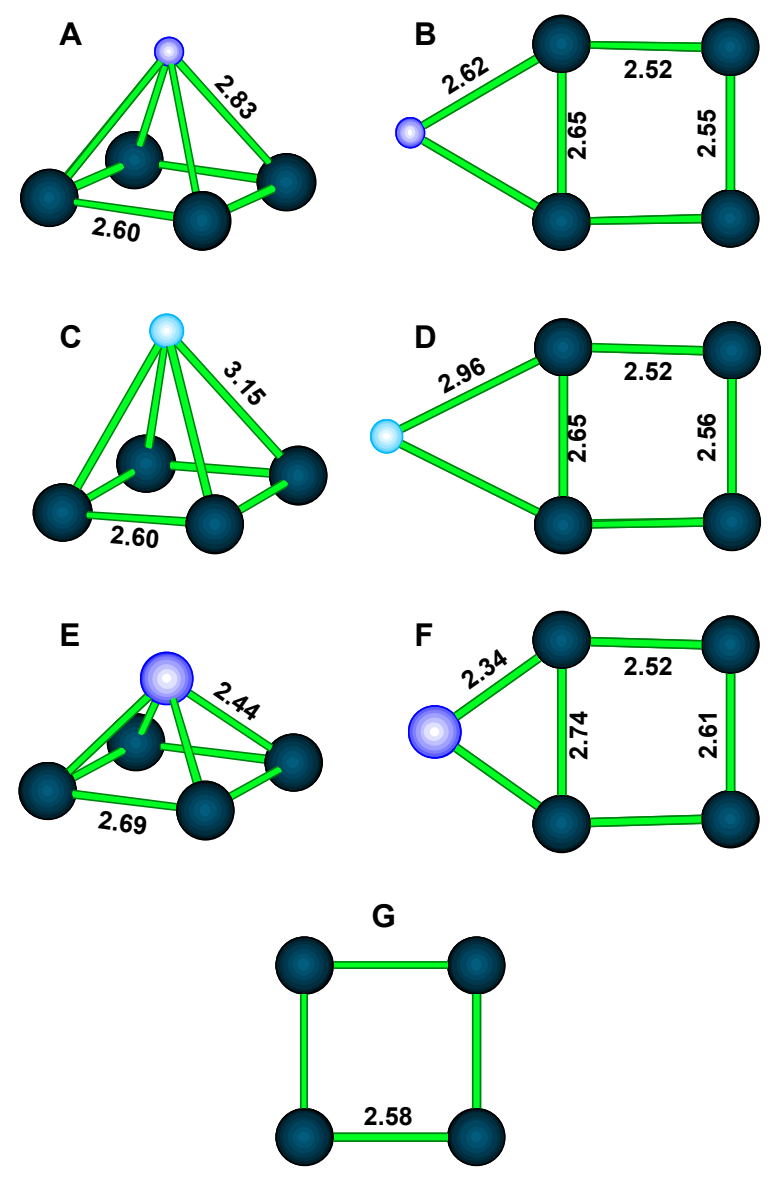

Figure 4.5. Optimized structures of $\mathrm{MAl}_{4}^{-}(\mathrm{M}=\mathrm{Li}, \mathrm{Na}$, $\mathrm{Cu})$ and $\mathrm{Al}_{4}{ }^{2-}$. 


\section{Experimental and Theoretical Observations of Aromaticity in Heterocyclic $\mathrm{XAl}_{3}^{-}(\mathrm{X}=\mathrm{Si}, \mathrm{Ge}, \mathrm{Sn}, \mathrm{Pb})$ Systems}

\section{(Li et al. 2001b)}

We performed a combined experimental and theoretical investigation of a series of Al-mixed clusters, $\mathrm{XAl}_{3}{ }^{-}(\mathrm{X}=\mathrm{Si}, \mathrm{Ge}, \mathrm{Sn}, \mathrm{Pb})$. We extended the concept of aromaticity into the hetero-cyclic four-membered ring all-metallic systems, $\mathrm{XAl}_{3}{ }^{-}$. We showed systematically the importance of the delocalization of the $\pi$ electrons for the stability of the cyclic aromatic structure. Metallic systems can be aromatic when they are composed of hetero-atoms, provided that the heteroatoms have electronegativities relatively similar to the rest of the ring. However, if the hereto-atoms differ substantially in their electronegativities, such as $\mathrm{C}$ in $\mathrm{CAl}_{3}{ }^{-}$, the cyclic aromatic structure is no longer a minimum.

\section{Photoelectron Spectroscopy of Mono-Niobium Carbide Clusters $\mathrm{NbC}_{n}{ }^{-}$ $(n=2-7)$ : Evidence for a Cyclic to Linear Structural Transition}

\section{(Zhai et al. 2001)}

We investigated a series of mono-niobium carbide clusters, $\mathrm{NbC}_{\mathrm{n}}^{-}(n=2-7)$, using anion photoelectron spectroscopy (Figure 4.6). Vibrationally resolved photoelectron spectra were observed for $\mathrm{NbC}_{2}{ }^{-}$and $\mathrm{NbC}_{3}{ }^{-}$, which were both shown to have cyclic $\mathrm{C}_{2 \mathrm{v}}$ structures. Two isomers were observed for $\mathrm{NbC}_{4}{ }^{-}$and $\mathrm{NbC}_{5}{ }^{-}$. The weak and low electron binding energy isomers were shown to be cyclic structures forming a series with $\mathrm{NbC}_{2}{ }^{-}$and $\mathrm{NbC}_{3}{ }^{-}$and all have similar and low electron binding energies. The main isomers of $\mathrm{NbC}_{4}{ }^{-}$and $\mathrm{NbC}_{5}{ }^{-}$, which possess much higher electron binding energies, were shown to be due to linear structures, which form a series with $\mathrm{NbC}_{6}^{-}$and $\mathrm{NbC}_{7}^{-}$. All the linear $\mathrm{NbC}_{\mathrm{n}}{ }^{-}$clusters were observed to have high electron binding energies and exhibit an evenodd alternation, similar to that observed for pure linear carbon clusters in the same size range. A cyclic to linear structural transition was thus observed for the $\mathrm{NbC}_{\mathrm{n}}^{-}$clusters from $\mathrm{NbC}_{3}{ }^{-}$to $\mathrm{NbC}_{4}^{-}$with the cyclic structures favored for the smaller clusters and the linear isomers favored for the larger clusters.

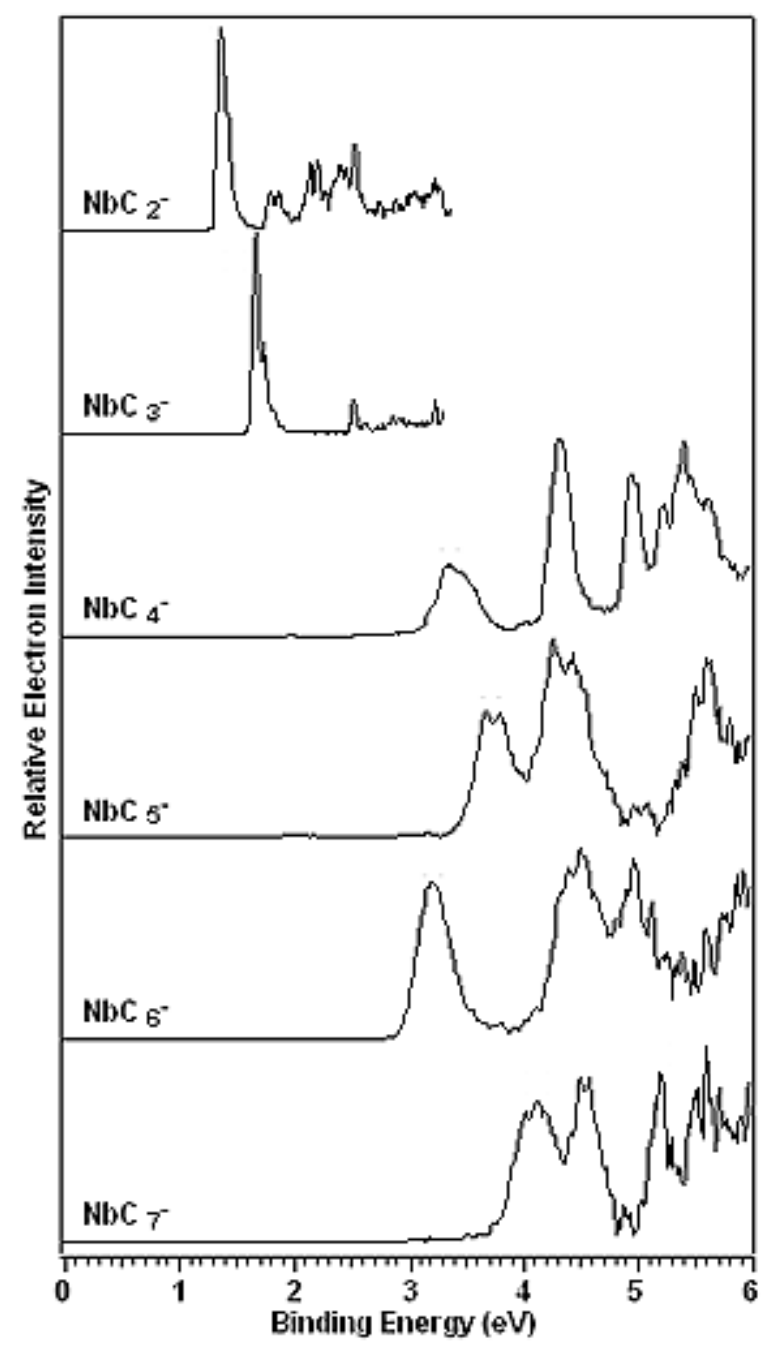

Figure 4.6. Photoelectron spectra of $\mathrm{NaC}_{\mathrm{n}}^{-}(n=2-7)$.

\section{On the Aromaticity of Square Planar $\mathrm{Ga}_{4}{ }^{2-}$ and $\mathrm{In}_{4}{ }^{2-}$ in Gaseous $\mathrm{NaGa}_{4}{ }^{-}$and $\mathrm{Naln}_{4}^{-}$Clusters}

\section{(Kuznetsov et al. 2001a)}

We investigated the electronic structure and chemical bonding of two bimetallic clusters, $\mathrm{NaGa}_{4}{ }^{-}$and $\mathrm{NaIn}_{4}{ }^{-}$. Photoelectron spectra of the anions were obtained and compared with ab initio calculations. We found that the ground state of the two anions contains a square planar dianion interacting with a $\mathrm{Na}^{+}$cation. The $\mathrm{Ga}_{4}{ }^{2-}$ and $\mathrm{In}_{4}{ }^{2-}$ dianions both possess two delocalized $\pi$ electrons and are considered to be aromatic, similar to that recently found in $\mathrm{Al}_{4}{ }^{2-}$. Using calculations for a model compound, we showed that a recently synthesized $\mathrm{Ga}_{4}$-organometallic compound also contains an aromatic $\mathrm{Ga}_{4}{ }^{2-}$ - unit, analogous to the gaseous clusters. 


\section{Aromatic Mercury Clusters in Ancient Amalgams}

\section{(Kuznetsov et al. 2001b)}

Mercury forms a wide range of alloys (amalgams), some of which were known from ancient times. In many alkali metal amalgams, mercury tends to nucleate into clusters or ribbons, depending on the mercury content. The square-planar $\mathrm{Hg}_{4}{ }^{6-}$ unit has been found to be a particularly favorable building block in alkali metal amalgams with a $3 / 2 \mathrm{M} / \mathrm{Hg}$ ratio. Why $\mathrm{Hg}_{4}{ }^{6-}$ is such a stable building block and why it assumes a perfect square structure are questions that have not been answered. We showed that these unusual properties of $\mathrm{Hg}_{4}{ }^{6-}$ are actually derived from aromaticity. We found, through a theoretical analysis of an isolated $\mathrm{Na}_{6} \mathrm{Hg}_{4}$ species, that $\mathrm{Hg}_{4}{ }^{6-}$ is aromatic, analogous to the recently discovered all-metal aromatic $\mathrm{Al}_{4}{ }^{2-}$ (Figure 4.7).
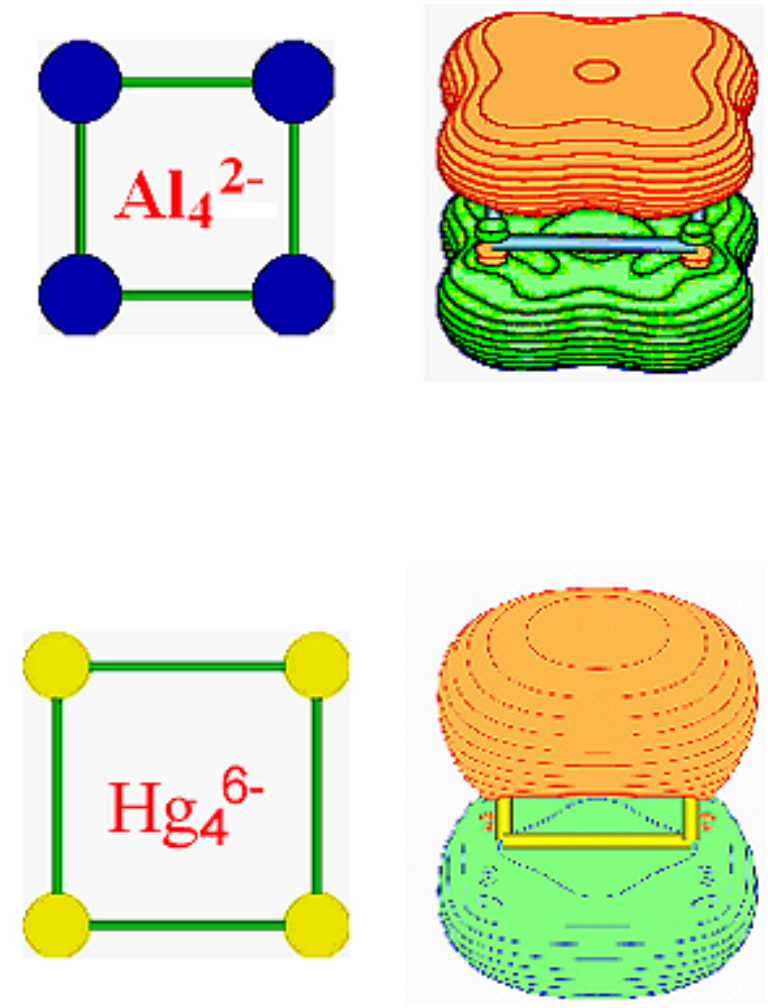

Figure 4.7. The square-planar structures of $\mathrm{Al}_{4}{ }^{2-}$ and $\mathrm{Hg}_{4}{ }^{6-}$, and their $\pi$ orbitals.

\section{References}

Kuznetsov, A. E., A. I. Boldyrev, X. Li, and

L. S. Wang, J. Am. Chem. Soc. 123, 8825-8831 (2001a).

Kuznetsov, A. E., J. D. Corbett, L. S. Wang, and A. I. Boldyrev, Angew. Chem. Int. Ed. 40, 33693372 (2001b).

Li, X., A. E. Kuznetsov, H. F. Zhang, A. I. Boldyrev, and L. S. Wang, Science 291, 859-861 (2001a).

Li, X., H. F. Zhang, L. S. Wang, A. E. Kuznetsov, N. A. Cannon, and A. I. Boldyrev, Angew. Chem. Int. Ed. 40, 1867-1870 (2001b)

Zhai, H. J., S. Liu, X. Li, and L. S. Wang, J. Chem. Phys. 115, 5170-5178 (2001).

\section{High Resolution Infrared Spectroscopy of Small Molecular Clusters}

\author{
T. A. Blake, R. L. Sams, \\ S. W. Sharpe, and S. S. Xantheas ${ }^{(a)}$
}

Supported by DOE.
(a) Pacific Northwest National Laboratory, Willilam R. Wiley Environmental Molecular Sciences Laboratory.

The cooling achieved in supersonic molecular beams can be used to enhance the formation of molecular clusters. The study of such small clusters provides insights into the nature of intermolecular interactions, which determine, for example, the structures of liquids and solids, control chemical reactions and proton exchange, and influence bio-molecule activity. We have ongoing projects to record and analyze the infrared spectra of several different molecular clusters. These include water clusters, clusters involving carbon dioxide and nitrous oxide, and hydrogen fluoride clusters. These spectra were recorded using either our cw slit-jet/Fourier transform infrared spectrometer or our pulsed slit-jet/infrared diode laser spectrometer.

The importance of liquid water as a solvent and moderator of biological activity is unquestioned. Consequently, its chemical and physical properties 
have received close study for many years. One course of research has been to examine progressively larger water cluster sizes using high-resolution spectroscopy. Recently (Goss et al. 1999) we successfully recorded low resolution survey spectra of larger water clusters using a Fourier transform infrared spectrometer to interrogate our cw slit-jet molecular beam source. The clusters were formed by flowing argon through a heated container of water to produce a 33 percent water-in-argon mixture that was expanded through the $12 \mathrm{~cm} \times 50 \mu \mathrm{m}$ slit. The survey spectra showed cluster absorption features for intermolecular librational modes, bending, and free and bound $\mathrm{OH}$ stretching vibrations. Based on intensity profiles, we estimated that the water clusters sizes ranged between 10 and 100 water molecules. Since the cluster sizes were so large, no rotational structure could be observed when the cluster absorption features were scanned under high resolution $\left(0.0015 \mathrm{~cm}^{-1}\right)$. With several improvements made to increase detection sensitivity, Prof. Lisa Goss of Idaho State University made several attempts during 2001 to scan through the water cluster features again using high resolution. This time, however, the water concentration in the flowing mixture were lowered to reduce cluster size. Unfortunately, no high resolution spectra of smaller water clusters were observed despite attempts to vary the expansion backing pressure and temperature, carrier gas (argon, helium, and nitrogen), and water concentration ( 5 percent to 30 percent). But since we now know where the clusters absorption features are from the low-resolution survey scans in the cw slit-jet FTIR experiments, we believe that these experiments could be successfully done using our pulsed slitjet/diode laser spectrometer, which has greater detection sensitivity than the FTIR spectrometer.

One of the advantages that the cw slit jet/FTIR spectrometer has over the diode laser system is that the FTIR provides broad spectral coverage. High resolution infrared spectra of small molecule clusters such as those involving carbon dioxide and nitrous oxide have been studied in the past using either diode or color center lasers. These lasers have high spectral brightness, but do not always provide seamless spectral coverage. We have recently examined asymmetric stretch spectra of the clusters $\mathrm{N}_{2} \mathrm{O}-\mathrm{N}_{2} \mathrm{O}, \mathrm{N}_{2} \mathrm{O}-$ $\mathrm{CO}_{2}, \mathrm{CO}_{2}-\mathrm{CO}_{2}, \mathrm{Ar}-\mathrm{N}_{2} \mathrm{O}$, and $\mathrm{Ar}-\mathrm{CO}_{2}$ using the $\mathrm{cw}$ slit jet/FTIR spectrometer. The spectrum recorded of $\mathrm{N}_{2} \mathrm{O}-\mathrm{N}_{2} \mathrm{O}$, for example, included five hundred transitions that were assigned and fit to a semi-rigid rotor Hamiltonian compared to 157 transitions that were assigned and fit from a diode laser spectrum (Qian 1997) (see Figure 4.8). Our fit constants include the first values for the quartic distortion constants for this small cluster. In fact, the quality of the fit of our data is approximately three times better than that of the diode laser data fit (RMS [obs - calc] $=$ $0.00015 \mathrm{~cm}^{-1}$ for our FTIR data fit vs. $0.00045 \mathrm{~cm}^{-1}$ for the diode laser data fit). Analysis of the fit centrifugal distortion constants to determine the van der Waals stretching frequencies for this cluster is continuing.

The vapor phase of hydrogen fluoride exhibits a number of superlative thermodynamic properties such as its high heat capacity and high thermal conductivity. It has long been assumed that these properties are due to the formation of hydrogen bonded oligimers. In fact, the gas phase average molecular weight was measured to be close to $60 \mathrm{amu}$ (Sahm 1995). Consequently, small clusters of hydrogen fluoride $\left([\mathrm{HF}]_{\mathrm{n}}\right.$ with $\mathrm{n}<8$ ) have been of considerable theoretical interest over the past two decades and recent theoretical results have predicted planar, oblate symmetric top structures $\left(C_{n h}\right.$ symmetry) for the trimer, tetramer, and pentamer (Maerker et al. 1997) at the global minimum of their potential energy surfaces. These clusters are strongly bonded: the tetramer, for example, has a dissociation energy $\left(D_{0}\right)$ of over $7000 \mathrm{~cm}^{-1}$ with respect to fragmentation into separate monomers. As a result of this deep well, there are a number of intermolecular vibrational modes that should be accessible to the now-standard molecular beam-infrared laser techniques, but, except for the dimer, there has been little detailed (that is, rotationally resolved) experimental information for these complexes. Using our pulsed, slit-jet infrared diode laser spectrometer, we have recorded three rotationally resolved bands in the 700 to $800 \mathrm{~cm}^{-1}$ region. An intense beam of clusters is formed by expanding a 4 percent mixture of hydrogen fluoride in helium through a slit nozzle into a vacuum chamber. The beam is interrogated with the output of a lead-salt infrared diode laser. The jet absorption spectrum, along with a reference frequency spectrum, is digitized and recorded by computer. One of the observed bands is an out-of-plane $\mathrm{H}-\mathrm{F}$ torsion 


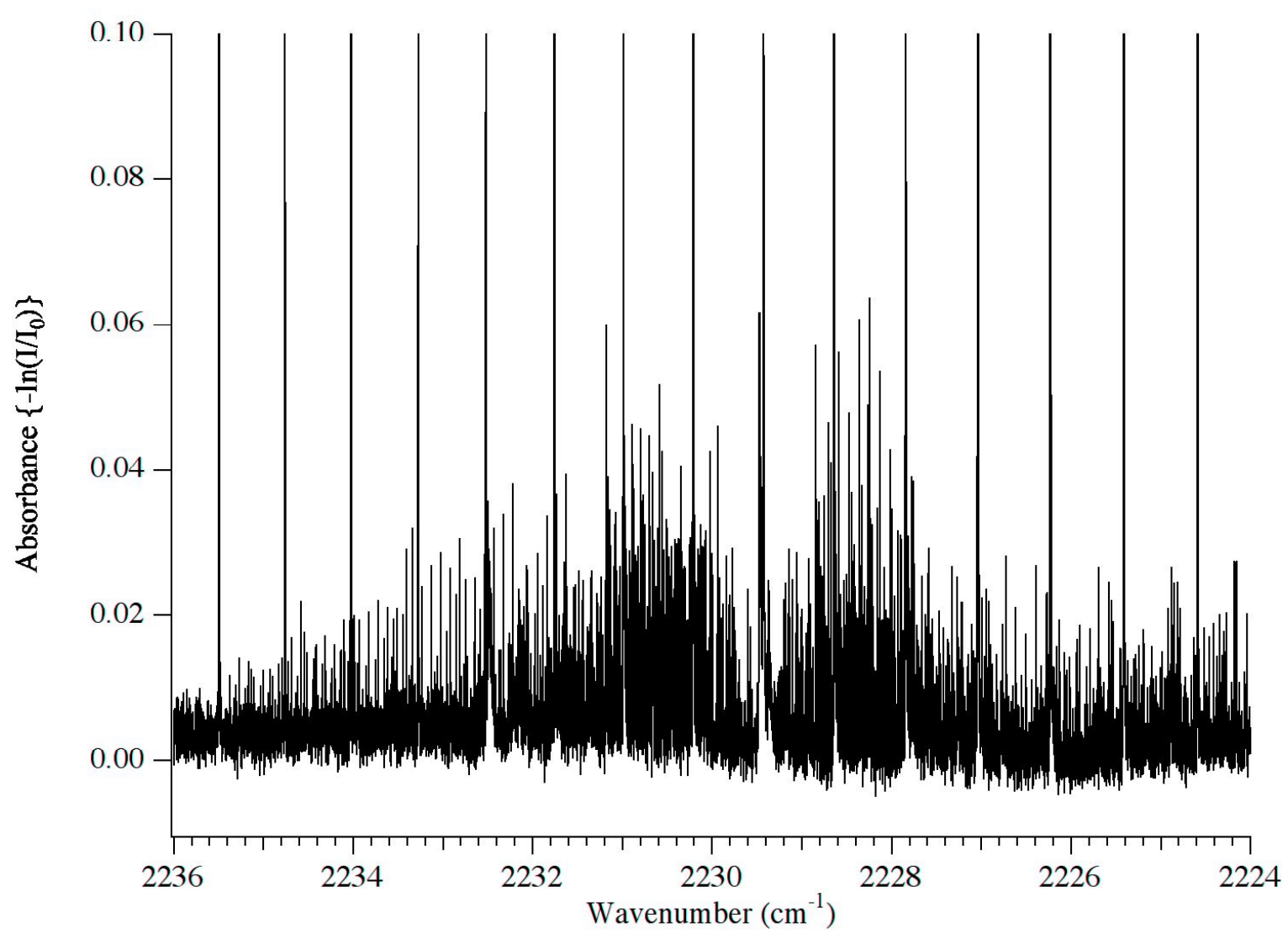

Figure 4.8. High-resolution infrared spectrum of nitrous oxide dimer recorded with a Fourier transform infrared spectrometer. Asymmetric stretch region. Evenly spaced, strongly absorbing peaks are nitrous oxide monomer peaks. The dimer was generated by expansion of a mixture of nitrous oxide and argon through a $12 \mathrm{~cm} \times 50 \mu \mathrm{m}$ slit jet.

fundamental $\left(A^{\prime \prime}\right)$ of hydrogen fluoride pentamer centered at 740.9701(3) $\mathrm{cm}^{-1}$. The band is indeed of a planar symmetric top ( $C_{5 h}$ symmetry), as predicted, with a measured ground state rotational constant, $B^{\prime \prime}$, of $0.07579(2) \mathrm{cm}^{-1}$; the predicted $B_{e}$ value is $0.07667 \mathrm{~cm}^{-1}$ (Maerker et al. 1997) (see Figure 4.9).

The two other bands have been assigned to the hydrogen fluoride tetramer: a perpendicular band centered at $752.7 \mathrm{~cm}^{-1}$ is assigned as an in-plane $\mathrm{H}-\mathrm{F}$ torsion fundamental $\left(E_{u}\right)$ and a parallel band centered at $714.7849(1) \mathrm{cm}^{-1}$ is assigned as an out-of-plane $\mathrm{H}-\mathrm{F}$ torsion fundamental $\left(A_{u}\right)$. Though resolved, the tetramer perpendicular band is quite complex and difficult to assign; but a preliminary assignment and fit of the rotational structure gives a ground state $B^{\prime \prime}$ value of $0.132 \mathrm{~cm}^{-1}$. The parallel band fits very well to a semirigid-rotor Hamiltonian and gives a groundstate $B^{\prime \prime}$ value of $0.132081(7) \mathrm{cm}^{-1}$. The predicted $B$ value for the tetramer is $0.1358 \mathrm{~cm}^{-1}$
(Maerker et al. 1997). Data and analysis of the tetramer parallel band has been recently published (Blake et al. 2000). We are presently extending our spectral searches for other intermolecular bands of $(\mathrm{HF})_{\mathrm{n}}$ and $(\mathrm{DF})_{\mathrm{n}}$ with $\mathrm{n}=3-6$.

\section{References}

Blake, T. A., S. W. Sharpe, S. S. Xantheas, J. Chem. Phys. 113, 707 (2000).

Goss, L. M., S. W. Sharpe, T. A. Blake, V. Vaida, and J. W. Brault, J. Phys. Chem. A 103, 8620 (1999).

Maerker, C., P. R. Schleyer, K. R. Liedl, T.-K. Ha, M. Quack, and M. A. Suhm, J. Comp. Chem. 18, 1695 (1997).

Qian, H., W. A. Herrebout, and B. J. Howard, Mol. Phys. 91, 689 (1997).

Suhm, M. A, Ber. Bunsenges. Phys. Chem. 99, 1159 (1995). 


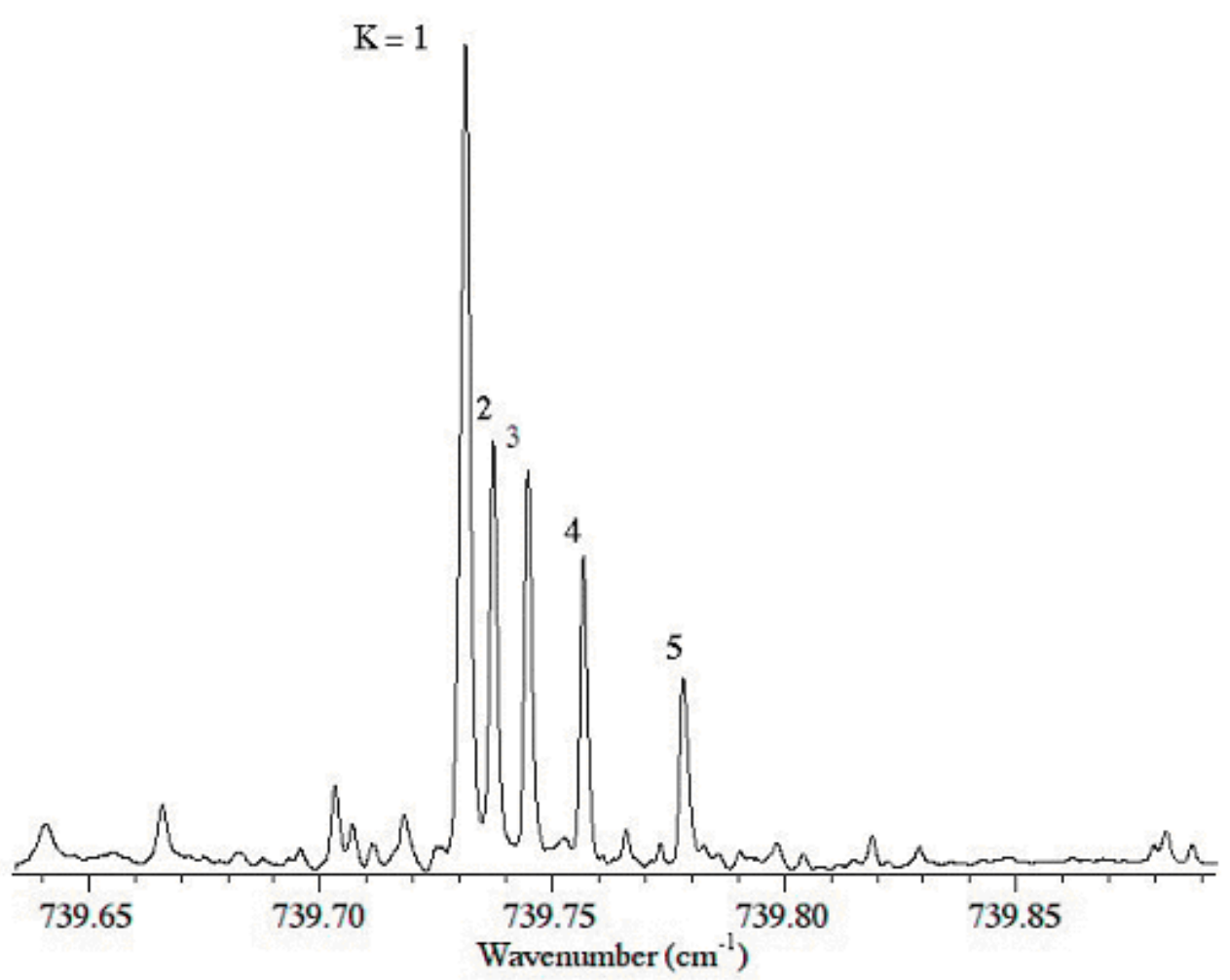

Figure 4.9. Hydrogen fluoride pentameter, $H-F$ out-of-plane libration $(A "), p(g)$ transition. $K$ substructure of the $P(8)$ rovibrational transition of the $\mathrm{H}-\mathrm{F}$ out-of-plane librational mode of hydrogen fluoride pentamer, recorded using a lead-salt infrared diode laser. The cluster was formed by expanding a $4 \% \mathrm{HF}$-in-helium mixture through a $12 \mathrm{~cm} \times 200 \mu \mathrm{m}$ pulsed slit into a $1 \times 10^{-5}$ torr vacuum chamber. 


\section{Single-Molecule Spectroscopy and Biomolecular Sciences}

This research emphasizes single-molecule spectroscopy and high-resolution biological imaging techniques for studying biological systems. Recent advances in fluorescence microscopy, at PNNL and elsewhere, make it possible to detect single molecules at room temperature, and to conduct spectroscopic measurements to monitor their dynamic processes. We have demonstrated fluorescence imaging of single molecules by two-photon excitation with a femto-second laser. This has the advantages that the excitation volume is small, the penetration is deep, and photo-damage is reduced for biological samples, offering the opportunity of viewing chemical reactions in a living cell in real-time. Structures are known for many proteins that perform vital cell functions, including DNA damage repair, reaction catalysis, and cell signaling. However, how they perform these functions is generally not known. Single-molecule and single-cell measurements provide real-time data on the molecular motions involved during these functions and how the timing of these reactions is correlated with other cellular biological activities. These data are likely to produce new information otherwise hidden, and will open up exciting possibilities for probing cellular processes.

Another research area is that of cellular response to low-dose radiation at levels below standard detection limits. A novel spatially-resolved cell irradiator allows the selective irradiation of individual cells to reveal the individual pathways that lead to radio-adaptive responses. This will enable researchers to understand the biological consequences and health effects associated with very low radiation doses. Such knowledge can help form more technically rigorous bases for human health and environmental protection standards. 


\section{Single-Molecule Nanosecond Anisotropy Dynamics of Tethered Protein Diffusion}

\author{
D. $\mathrm{Hu}^{(a)}$ and H. P. Lu
}

Supported by EMSL Operations and DOE Office of Basic Energy Sciences

(a) Postdoctoral Research Associate.

Confined and hindered protein motions are generally found in living cells, with tethered rotational motions of proteins or protein domains particularly associated with and relevant to the early events of molecular interactions in cell signaling at exterior- and innercellular membrane surfaces. Ensemble-averaged time-resolved fluorescence anisotropy (Cross and Fleming 1988) has been extensively applied to study the protein rotational and conformational motion dynamics under physiologically relevant conditions. However, the spatial and temporal inhomogeneities of the non-synchronizable stochastic protein rotational and conformational motions are extremely difficult for such ensemble-averaged measurements to characterize. We studied spatially confined rotational diffusion dynamics of individual tethered proteins on a hydrocarbon modified glass surface under water using a novel approach of single-molecule nanosecond anisotropy (Figure 5.1).

We have observed dynamic inhomogeneity of the rotational diffusion dynamics, i.e., diffusion rate fluctuation, due to interactions between the proteins and the surface. We have also found that the longtime averages of the dynamically inhomogeneous diffusion rates of single molecules are essentially homogeneous amongst the single molecules examined. Moreover, tethered proteins stay predominately in solution phase, rather than interacting with the modified surface, and the infrequent interactions are not energetic enough to fix the protein rotational motions. These conclusions imply that the tethered protein or protein domain motions on the exterior- and inner-cell membrane surfaces are dynamically inhomogeneous, even if the membrane surface or the local environment are homogeneous; in contrast, the static inhomogeneity of the rotation dynamics can only exist when the local membrane surface or the local environment are inhomogeneous.

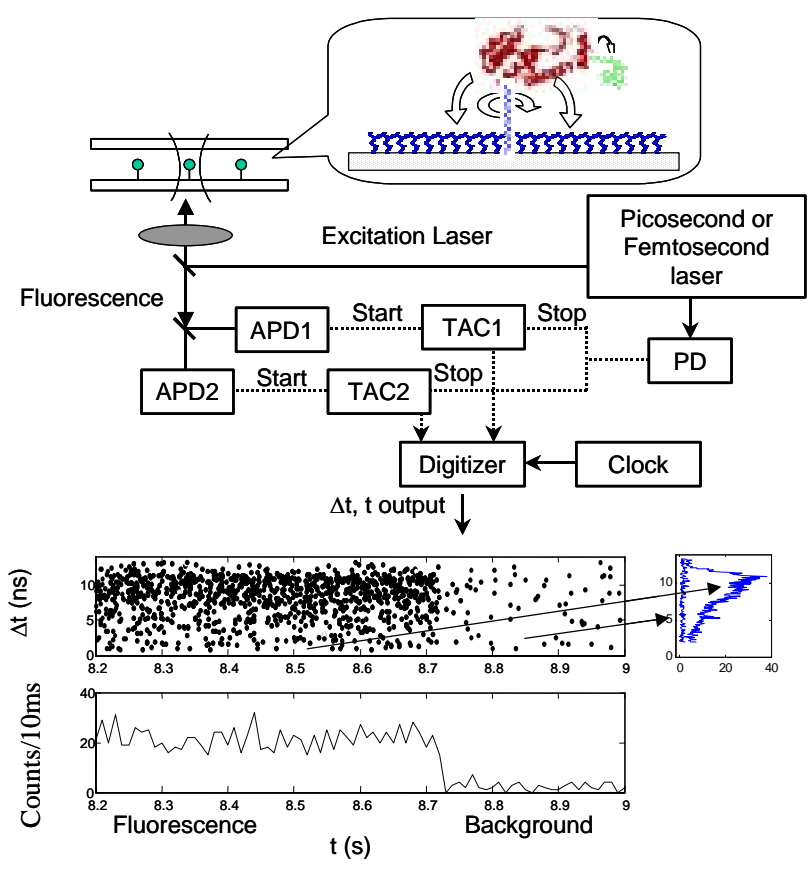

Figure 5.1. Schematic of a single-molecule confocal microscope with two-channel photon time-stamping TCSPC. It allows us to study the fluorescence intensity, lifetime, and anisotropy of a single molecule simultaneously by recording the arrival time and delay time of each fluorescence photon. The middle left plot is an example of the raw data of the photon timestamping TCSPC of a detector channel. Each dot corresponds to a photon plotted by its arrival time ( $\mathrm{t}$ ) and delay time $(\Delta t)$. The fluorescence intensity trajectory (bottom plot) is calculated from the histogram of arrival time (t) with 0.01 -second time-bin resolution. The molecule was photo-bleached at 8.71 seconds. The nanosecond fluorescence decay curves (top-right plot) are the histograms of the delay time of the fluorescence photons $(t<8.1)$ and background photons $(\mathrm{t}>8.1)$.

The new approach of combining photon-time stamping (Fries et al. 1998) and single-molecule nanosecond anisotropy demonstrated and applied in our work allows measurement of single-molecule rotational motion dynamics in a wide time-scale from nanoseconds to seconds (Figure 5.1; Figure 5.2). This permits recording time trajectories of single-molecule rotational rate constants and time-zero anisotropy amplitudes. It also effectively decouples the measurement of anisotropy decay time fluctuation from that of the fluorescence lifetime fluctuation. 

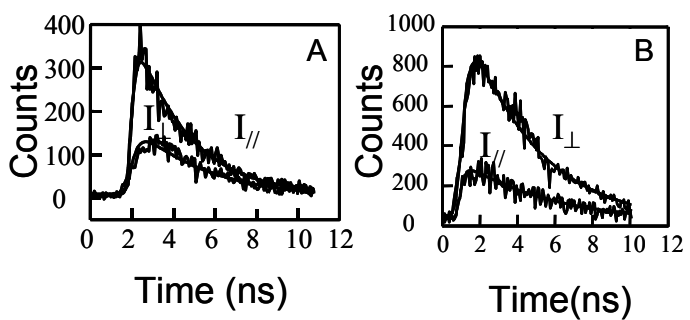

Figure 5.2. The parallel and perpendicular fluorescence decay and single exponential fits of a single T4 lysozyme/Alexa 488 molecule covalently linked to surface $(A)$ and a single rhodamine 123 molecule on PMMA surface (B).

\section{References}

Cross, A. J. and G. R. Fleming, Biophys. J. 50, 507 (1986).

Fries, J. R., L. Brand, C. Eggeling, M. Kollner, and C.A.M. Seidel, J. Phys. Chem. 102, 6601 (1998).

\section{Single-Molecule Dynamics of Protein-DNA Interactions in DNA Damage Recognition}

\author{
H. Peter Lu, Lilia M. Iakoucheva, ${ }^{(a, b)}$ \\ and Eric J. Ackerman ${ }^{(b)}$ \\ Supported by Laboratory Directed Research and \\ Development (LDRD) funding and DOE Office \\ of Basic Energy Sciences. \\ (a) Postdoctoral Research Associate. \\ (b) PNNL, Molecular Biosciences Department.
}

DNA damage recognition is an essential early step in DNA repair. Understanding the molecular basis for numerous subtle interactions amongst proteins and complexes with DNA is the foundation for designing reliable models of the repair process. It is crucial to obtain quantitative data about the dynamic parameters regulating repair protein-DNA interactions; e.g., time, interaction distances, changes in bend angles of DNA, binding strengths, and stochiometric effects (Lu et al. 2001). The proteins and substrates characterized in this manner can then be analyzed by single-molecule spectroscopy, atomic force microscopy (AFM) (Lu et al. 1998; Lu and Xie 1997), and fluorescence imaging microscopy (Figure 5.3). Thus, combined information can be obtained at the ensemble level as well as at the single-molecule level, maximizing our depth of understanding. A vivid, testable picture

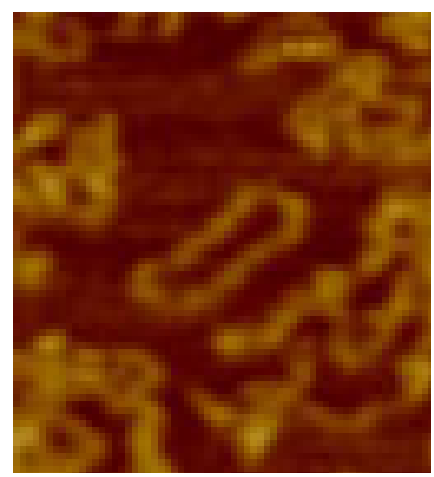

Figure 5.3. AFM imaging DNA plasmid pUC-18 on mica under buffer solution using an Au-coated AFM tip. Significant conformational inhomogeneity is evident. This imaging capability will be combined with single-molecule spectroscopic and imaging capability to achieve combined measurements on DNA or DNAXPA molecules.

of the controlling first step in repair, damage recognition, will provide a worthwhile beginning.

Our single-molecule spectroscopy study (Lu et al. 2001) reveals the dynamics of fluctuating molecular noncovalent interactions within single protein-DNA complexes involving in the damage-recognition process in DNA repair. Xeroderma pigmentosum group-A protein (XPA) is involved in recognizing DNA lesions. Our experiments used recombinant, fulllength Xenopus XPA and a double-stranded (ds) 55-mer oligonucleotide with fluorescein covalently attached at a 5'-end. Fluorescein is a lesion for DNA repair, and fluorescence quenching is observed upon XPA binding.

We observed fluorescence intensity fluctuations in single-XPA-DNA complexes at two different time scales (Lu et al. 2001). The slow intensity fluctuation amplitude change is comparable to that of the bound and unbound states of XPA-DNA observed by conventional fluorescence spectroscopy. In single-molecule experiments, fluorescence fluctuations reflect conformational changes between the bound and loosely bound states while the overall complex is still associated. We attribute the fast fluctuations to conformational changes in DNA and the slow fluctuations to the XPA-DNA interactive motions. Slow and largeamplitude conformational motions by DNA-XPA complexes have not been reported previously and this finding is consistent with XPA's ability to recognize a wide variety of DNA lesions. 
Approximately 10-fold variation occurs in the rates of the slow interactive conformational motions among the individual complexes (Figure 5.4). This inhomogeneity is most likely associated with the existence of different subsets of protein conformations seeking an induced-fit to a conformationaly fluctuating DNA lesion. These slow-fluctuating interactions could be crucial in XPA-DNA interaction dynamics and intrinsically pertinent to the protein-DNA complexes in other DNA damage recognition processes. This hypothesis is currently being evaluated by using different types of DNA with site-specific lesions (lesion in the middle of double-stringed and plasmid DNAs) and XPA mutants with targeted modifications.

Our single-molecule approaches reveal detailed DNAprotein and protein-protein dynamics that are unobtainable by conventional ensemble-averaged approaches due to the static and dynamic inhomogeneities and non-synchronizable conformational fluctuations of the DNA-protein interactions. The methodologies of single-molecule, real-time topologi$\mathrm{cal} /$ optical imaging and spectroscopy that are developed in this research will be widely applicable to understanding the general interaction mechanisms of biological complexes, e.g., the transcription, replication, and signal transduction networks involved in cancer.

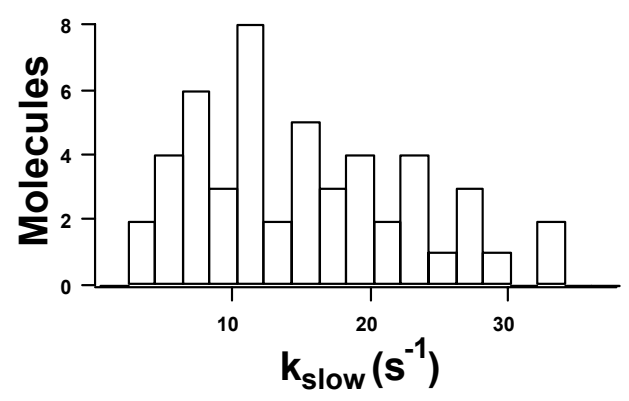

Figure 5.4. Distribution of the slow XPA-DNA binding-unbinding nuclear motion rates for 50 individual complexes. A 20 -fold variation of the XPA binding-unbinding motion rate is observed, and the distribution is peaked at $11 \pm 3 \mathrm{~s}^{-1}$.

\section{References}

Lu, H. P., L. Xun, and X. S. Xie, Science 282, 1877 (1998).

Lu, H. P., L. M. Iakoucheva, and E. J. Ackerman, J. Am. Chem. Soc. 123, 9184 (2001).

Lu, H. P. and X. S. Xie, Nature 385, 143 (1997).

\section{Revealing Function-Regulating Conformational Dynamics of an lon Channel by Simultaneous Single-Molecule Fluorescence Imaging and Patch-Clamp Recording}

\author{
G. Harms, ${ }^{(a)}$ G. Orr, B. Thrall, ${ }^{(b)}$ S. Colson, \\ M. Montal, ${ }^{(c)}$ and H. P. Lu
}

Supported by Laboratory Directed Research and Development (LDRD) funding.

(a) Postdoctoral Research Associate.

(b) PNNL, Molecular Biosciences Department.

(c) UCSD, Department of Biology.

Ion channels control and regulate the ion current flux and therefore play a key role in regulating how cells respond to their changing environment. We have been developing a unique technique by combining our extensive imaging capabilities, a confocal scanning linear/non-linear optical microscope, with state-of-theart patch clamp technologies. Application of this unique instrumentation presents an unprecedented opportunity of seeking a mechanistic and dynamic understanding of ion channel functions and structures in living cells.

Subtle conformational changes of ion channels control gating and selectivity (Ferrer-Montiel et al. 1996).

Structure-function studies of ion channels at the single molecule level are needed to identify such conformational changes within the membrane that govern their dynamic activity. We have used single-molecule fluorescence spectroscopy (Lu et al. 1998) simultaneously with single-channel current recordings (Sakmann and Neher 1998), to detect discrete structural changes simultaneously with fluctuations in channel activity of single gramicidin channels in a lipid bilayer membranes and single NMDA ion channel in a living cell (Figure 5.5). Single pair fluorescence resonant energy transfer (spFRET) maging and single-molecule polarization imaging experiments and single-channel current correlation analysis revealed the correlation of multiconformational states with multiconductance levels of the channel. Our findings suggest that the inhomogeneous, dynamic conformational changes of the gramicidin channel underlie the correlation of the structural changes with the channel conductance. 


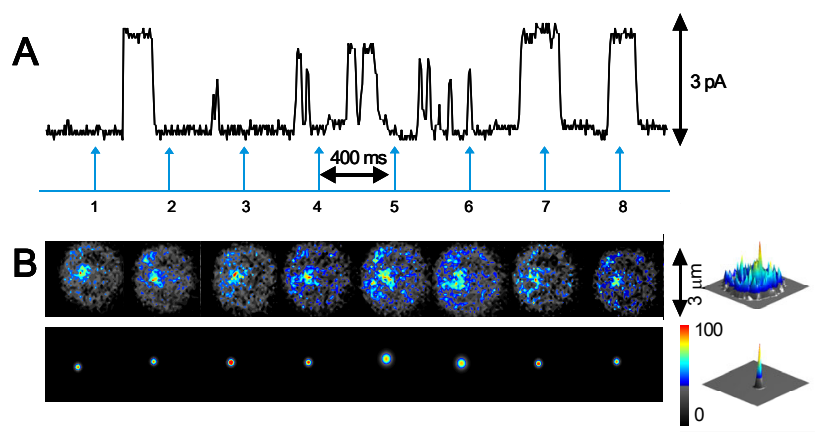

Figure 5.5. A) Upper trace: single channel current recorded at $100 \mathrm{mV}$ from a TMR labeled single gramicidin channel incorporated in a lipid bilayer. Lower trace: time flags indicating the $5 \mathrm{~ms}$ of laser excitation and CCD camera exposure simultaneously acquired with the single channel recording. B) top panel: consecutive $3 \mu \mathrm{m} \times 3 \mu \mathrm{m}$ fluorescence images of the pipette-tip taken simultaneously with the singlechannel current showed in A. TMR labeled gramicidin was excited with $514 \mathrm{~nm}$ for $5 \mathrm{~ms}$. The images were taken every $400 \mathrm{~ms}$ and were numbered according to the time flags in A. Bottom panel: plot of the 2-D Gaussian fitting for the above image. Right insert: 3-D plot of the first image (top) and 3-D plot of the 2-D Gaussian fitting (bottom).

Correlation function analysis of single channel currents supports the combined single channel fluorescence and conductance results by indicating multiple open/closed states and a dependence between the two states. From experimental evidence that has accumulated during the years, the multiple states may arise from intra-and inter-molecular conformational changes and geminate recombination.

We have also validated a novel technique and methodology of simultaneously probing ion-channel modespecific conformational change and its correlated channel trajectory, combining two real-time singlemolecule approaches. Ion channel structural dynamics and kinetics can now be studied site-specifically at the molecular level under physiologically relevant conditions. Refinements of this strategy will make it possible to study subtle conformational changes in the receptor-ligand complexes that control ligand-gated channel activity in intact excitable cells.

\section{References}

Ferrer-Montiel, A., W. Sun, and M. Montal, Biophys J. 71, 749-758 (1996).

Lu, H. P., L. Xun, and X. S. Xie, Science 282, $1877-$ 1882 (1998).

Sakmann, B. and E. Neher, in Single-Channel Recording, Plenum, ed. by B. Sakmann and E. Neher (1995).

\section{Single-Molecule Spectroscopy Studies of Dynamic Protein-Protein Interactions in Cellular Signaling}

X. Tan, ${ }^{(a)}$ K. Hahn, ${ }^{(b)}$ and H. P. Lu

Supported by Laboratory Directed Research and

Development (LDRD) funding

(a) Postdoctoral Research Associate.

(b) The Scripps Research Institute, San Diego.

Cdc42 is a single-subunit GTP-binding protein (GTPase) that acts as a molecular switch in signaling pathways that regulate diverse cellular responses (Kraynov et al 2000). Cdc42 interacts with a variety of targets, and the Wiskott-Aldrich syndrome protein (WASP) is postulated to link Cdc42 activation directly to actin rearrangement. Single-molecule spectroscopy is a powerful technique for probing conformational changes in protein interactions (Lu et al. 2001) and has the potential of providing molecular-level understanding of the intracellular signaling processes.

In this study, we are using CBD, a dye-labeled fragment from WASP, which binds only the active GTP-bound Cdc42, as a biosensor to report Cdc42 activity. The solvatochromic dye responds to the strong hydrophobic nature of the active Cdc42-CBD binding, as shown by the dramatic emission intensity increase in Figure 5.6.

Single Cdc42-CBD protein complexes at $\mathrm{nM}$ concentration were imbedded in agarose gel, sandwiched between two glass coverslips, and located with an inverted fluorescence confocal microscope by rasterscanning the sample with focused diffraction-limited laser at $568 \mathrm{~nm}$. We observed fluorescence intensity 


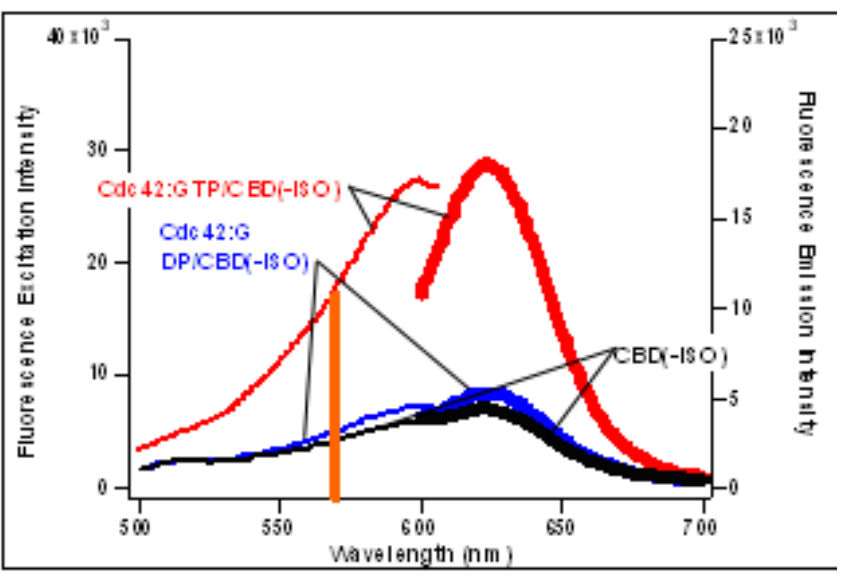

Figure 5.6. Excitation and emission spectra of dyelabeled protein-protein complexes. Ensemble fluorescence assays of Cdc42 interactions with CBD biosensor. CBD binds only the active GTP-bound Cdc42, which is demonstrated by dramatic increase in the dye emission intensity. A nonhydrolyzable GTP analogue, GTP- $\gamma-S$, is used to lock Cdc42 into the active form. Thin curves use left scale; thick curves use right scale. Vertical line marks excitation frequency.

fluctuations in single Cdc42-CBD complexes at different time scales. Most of the autocorrelation functions derived from single-complex trajectories show bi-exponential decays with a fast and a slow component (Figure 5.7). The slow fluctuation ( $150 \mathrm{~ms}$ to $10 \mathrm{~s})$ is spontaneous while the fast component $(<10 \mathrm{~ms})$ might be photon-induced.

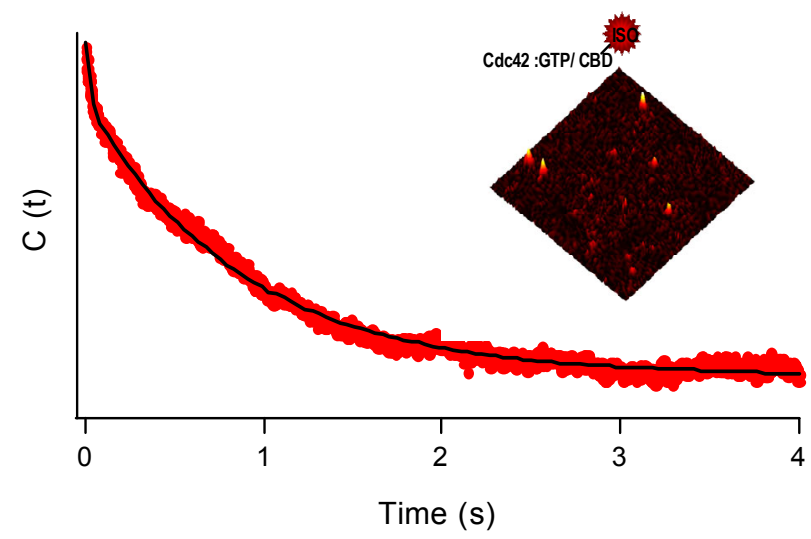

Figure 5.7. The autocorrelation function, $C(t)$, calculated from a fluorescence intensity trajectory of a single Cdc42-CBD protein complex. The solid line is a biexponential fit. Inset: Single-molecule fluorescence scanning image of individual Cdc42-CBD protein complexes.
We attribute the slow component, which occasionally splits into two, to the conformational fluctuations. We suggest the Cdc42-CBD binding is quite dynamic, giving rise to various conformations between the bound and loosely bound states where the overall complex is still associated. Fluorescence fluctuations in single-molecule experiments reflect the conformational changes between the bound and loosely bound states. The slow conformational fluctuation, likely associated with the interactive motions of the $\mathrm{Cdc} 42-$ CBD complex at the binding site, exhibits a wide variation in the rates among the individual complexes.

Further statistical analysis and single-molecule fluorescence anisotropy measurements will help unravel the dynamics of protein interactions in the $\mathrm{Cdc} 42$ -

CBD complex.

\section{References}

Lu, H. P., L. M. Iakoucheva, and E. J. Ackerman, J. Am. Chem. Soc. 123, 9184 (2001).

Kraynov, V. S., C. Chamberlain, G. M. Bokoch, M. A. Schwartz, S. Slabaugh, and K. M. Hahn, Science 290, 337 (2000).

\section{Surface Enhanced Raman Spectroscopy of Nanoparticles and Nanostructures: Towards Vibrational Mode-Specific Single- Molecule Electron Transfer Dynamics}

\author{
L. Zhu, ${ }^{(a)}$ G. Schenter, ${ }^{(b)}$ Y. D. Suh, ${ }^{(a)}$ and \\ H. P. $\mathrm{Lu}$
}

Supported by Laboratory Directed Research and Development (LDRD) funding and DOE Office of Basic Energy Sciences.

(a) Postdoctoral Research Associate.

(b) PNNL Theory, Modeling \& Simulation.

Single-molecule dynamics have been "traditionally" studied using fluorescence spectroscopy that probes the overall molecular or active site conformational fluctuations and the excited-state lifetimes.

Vibrational-mode resolved single-molecule dynamics have never been conducted due to the lack of vibrational specificity of fluorescence. Quite conceivably, Raman spectroscopy, including surface-enhanced 
Raman spectroscopy (SERS), could provide rich information on vibrational relaxation, vibrational coupling, chemical bond formation/dissociation, and mode-specific nuclear displacements.

In recent years, there have been tremendous advances in applying SERS to study nanoparticles and nanostructures at high sensitivity at the single-molecule level (Hongxing et al. 2000; Katrin et al 1999; Shuming and Emory 1997). To carry on the study of vibrational-mode resolved single-molecule dynamics, we have set up an atomic force microscopy (AFM) coupled SERS microscope system capable of simultaneously measuring topographic and spectroscopic information at nanometer scale or for a single nanoparticle (Figure 5.8). We have measured a series

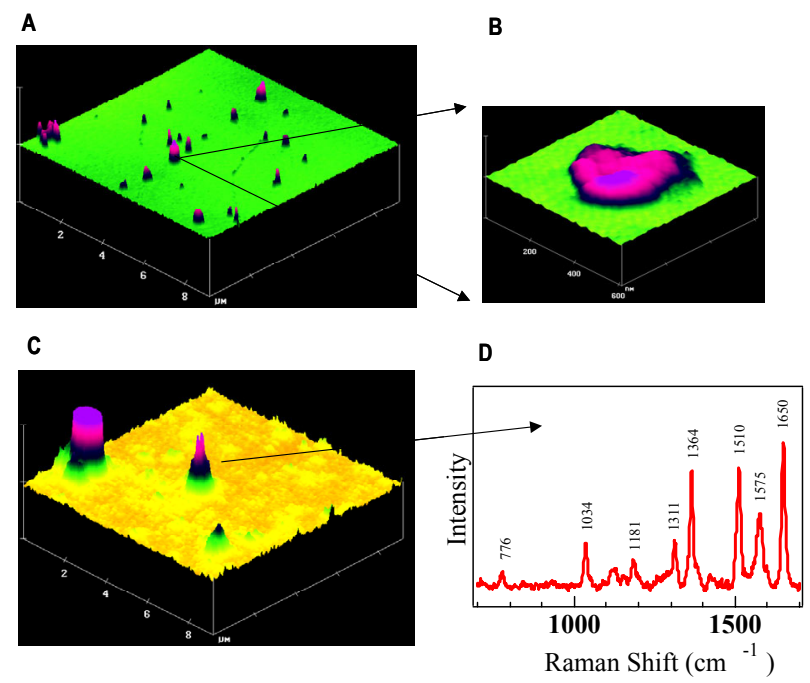

Figure 5.8. Correlated topographic and spectroscopic imaging and SERS spectroscopy of nanoparticles.

A) AFM image $\left(9 \times 9 \mu \mathrm{m}^{2}\right)$ of the rhodamine $6 \mathrm{G}$ molecules adsorbed on silver nanoparticles. B) AFM image zoom-in image of a single nanoparticle cluster from the imaging area of (A). C) 2-D Raman intensity image of the rhodamine $6 \mathrm{G}$ molecules adsorbed on silver nanoparticles at $514.5 \mathrm{~nm}$ excitation from the same area of (A). Each feature on the image is corresponded to a "hot" Raman spot. The Raman signal was detected by using a APD detector after a 30-nm-bandwidth bandpass filter at $564 \mathrm{~nm}$. D) SERS spectrum from the nanoparticle cluster imaged in $(A, B, C)$. of SERS spectra of R6G, myoglobin, cytochrome C, and hemoglobin proteins from single Ag nanoparticle clusters. It is observed that the peak intensities, peak/peak intensity ratios, and peak positions show fluctuating behaviors. Similar fluctuations have been reported as a signature of single-molecule SERS signals. However, our laser intensity dependent experimental results suggest that the fluctuations can even exist in multiple-dye-adsorbated nanoparticles at a high laser excitation intensity $\left(>\right.$ ca. $\left.100 \mathrm{~W} / \mathrm{cm}^{2}\right)$ and that the fluctuations are predominately photoinduced. In contrast, we also see spontaneous fluctuations at low laser excitation intensity $\left(<50 \mathrm{~W} / \mathrm{cm}^{2}\right)$.

The overall Raman intensity fluctuation is probably dictated by the change of the interaction of the molecules with the local electric field of a nanoparticle, for example, the rotational and translational motions of the adsorbed molecules under the near-field and gradient-field of the nanoparticles. The peak fluctuations are most likely associated with the change of molecular conformations reflecting the changes in the Franck-Condon coupling between the ground and excited states of the molecules. Computational modeling and simulation provide insights into the origins of the Raman spectral fluctuation. Quantitatively understanding these fluctuations will provide a foundation for applying SERS to study vibrational modespecific single-molecule dynamics to nanoscale systems.

\section{References}

Hongxing, X., E. J. Bjerneld, M. Käll, and L. Börjesson, Phy. Rev. Lett. 83, 4357-4360 (2000).

Katrin, K., H. Kneipp, I. Itzkan, R. R. Dasari, and M. S. Feld, Chem. Rev. 99, 2957-2975 (1999).

Shuming, N. and S. R. Emory, Science 275, 1102-1106 (1997). 


\section{Probing Single-Molecule Conformational Mode-Specific Dynamics under Enzymatic Reaction Conditions in Real Time}

\author{
Y. Chen, ${ }^{(a)}$ D. Hu, ${ }^{(b)}$ E. R. Vorpagel, ${ }^{(c)}$ and \\ H. P. Lu
}

Supported by EMSL Operations and DOE Office of Basic Energy Sciences.

(a) Visiting Scientist.

(b) Postdoctoral Research Associate.

(c) PNNL Theory, Modeling \& Simulation.

We have studied single-molecule enzymatic reaction dynamics and enzyme conformational motions of the T4 lysozyme during hydrolysis of the polysaccharide walls of $E$. coli $B$ cells. Although enzymatic reactions are traditionally studied at the ensemble level, the inhomogeneities of the reaction rate, the correlated enzyme conformational motions, and the nonsynchronized nature of these reactions make it extremely difficult to obtain stepwise mechanistic and dynamic information from such studies. Presumably, the protein conformational motions at the active site of the enzyme are most critically responsible for the inhomogeneities in the enzymatic reactions ( $\mathrm{Lu} \mathrm{1998,}$ 2001). However, there have been no real-time observations showing the correlation of single-molecule enzymatic reactions with unambiguously assigned protein conformational motions, which would be critical in understanding the mechanisms and dynamics of intrinsic inhomogeneous and non-synchronizable enzymatic reactions. Applying single-molecule spectroscopy, we are now able to probe protein conformational motions of a single enzyme (T4 lysozyme, an 18.6-kDa, single-subunit, and 164-amino acid enzyme comprising two domains connected by an $\alpha$-helix) during hydrolysis of the polysaccharide walls of $E$ coli $B$ cells.

Besides obtaining the rates for the specific steps of a complex and nonsynchronizable reaction, we find that the time required for the enzyme to locate a bindingsite dominates the inhomogeneity in the overall reaction rate and that the subsequent steps have homogeneous rates among the individual enzymes examined. These results are not obtainable for conventional ensemble-averaged experiments. Our results represent a step forward in achieving the potential for single-molecule methods to unravel the nature of biochemical processes within living cells. This is where reactions generally cannot be synchronized in ensemble-average mechanistic studies and where the inhomogeneous nature of living cells requires single-molecule measurements if the controling factors are to be understood and manipulated for health intervention or other biological purposes.

By attaching a donor-acceptor (tetramethylrhodamineTexas Red) pair of dye molecules site-specifically to non-interfering sites on the enzyme, we measured the dynamics of the hinge-bending motions of the enzyme under enzymatic turnovers by monitoring the donoracceptor emission intensity changes due to single-pair fluorescence resonance energy transfer (spFRET) (Figure 5.9). The overall enzymatic reaction rate

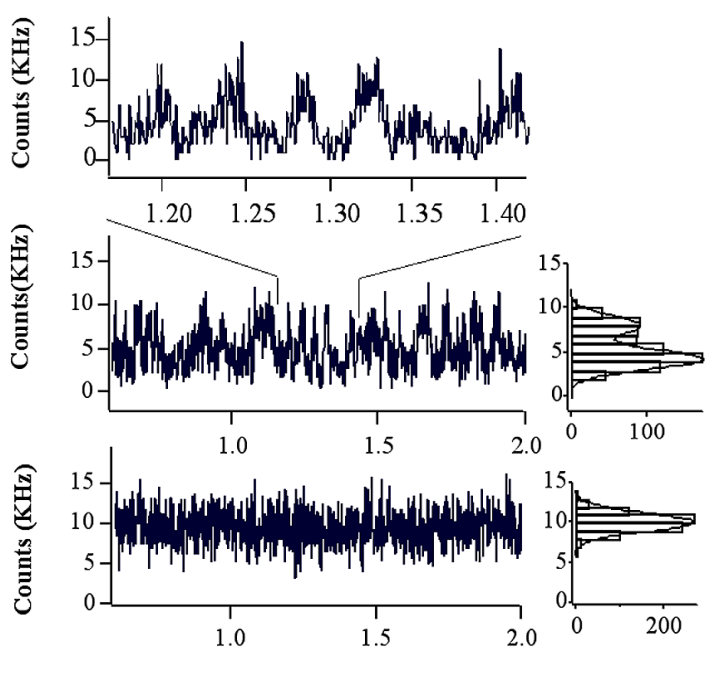

Time (s)

Figure 5.9. Real-time observation of single-T4 lysozyme conformational motions and enzymatic reaction turnovers during hydrolysis of bacterial cell walls. The data in the three panels were recorded at 0.65 ms per channel for the same reaction conditions. The upper panel shows an extended portion of middle-panel trajectory of the donor fluorescence of a donor-acceptor labeled single-T4 lysozyme. Intensity wiggles in the trajectory are evident. The lower panel shows a portion of a trajectory recorded from a donoronly-labeled protein. The fluorescence intensity distributions derived from the two trajectories are shown in the insets of the middle and lower panels. The solid lines are fits using single and bimodal Gaussian functions, respectively. The formation of ES and ES* involves significant domain breathing motions along the $\alpha$-helix "hinge" causing the large amplitude wiggles and the bimodal amplitude distribution. From this it can be seen that spFRET measurements are effective real-time probes of the enzymatic reaction dynamics. 
constants were found to vary widely from molecule to molecule. The enzyme's searching for reactive sites in the substrate was thought to account for most of this inhomogeneity. By applying a molecular dynamics (MD) simulation and a random-walk model to analyze the enzyme-substrate complex formation dynamics, we have revealed multiple intermediate conformational states in the chemical reaction process. This approach provides information on the microscopic conformational change mean drifting velocity, diffusion coefficient, friction coefficient, energy consumed by friction along the reaction coordinate, and the energy landscape. This information cannot be obtained by an ensemble-averaged experiment, but is obtainable by a single-molecule approach. Our combined approach is the first simultaneous measurement of enzyme conformational motion and enzymatic turnovers and demonstrates the potential of singlemolecule studies in understanding non-synchronizable, multi-step reaction dynamics and mechanisms.

\section{References}

Lu, H. P., L. Xun, and X. S. Xie, Science 282, 1877 (1998).

Lu, H. P., L. M. Iakoucheva, and E. J. Ackerman, J. Am. Chem. Soc. 123, 9184 (2001).

\section{Improving Image Quality in CARS Microscopy Using Spectroscopic Markers and New Optical Methods}

\author{
G. R. Holtom, B. D. Thrall, ${ }^{(a)}$ S. D. Colson, \\ and C.S. Chen ${ }^{(a)}$
}

Supported by Laboratory Directed Research and Development (LDRD) funding

(a) PNNL Molecular Biosciences.

Imaging the lipid distribution in membranes is a difficult problem, since the attachment of fluorescent probes can affect solubility and segregation of the construct. This is an ideal opportunity for applying the CARS imaging microscopy method. While the native molecules present in cells is useful for imaging, image contrast is low due to the small volume of membranes within a typical voxel under investigation. We are very interested in developing methods to improve the situation, and we may use both chemical and optical methods.
A very interesting possibility is using deuterated hydrocarbons. The fatty acid tails have very strong $\mathrm{C}$-D bonds that are not replaceable with hydrogen in the cellular environment, and these bonds have vibrational frequencies that are easily separable from the native hydrocarbons. In fact, virtually no native molecules have a resonance frequency in the range of $2120 \mathrm{~cm}^{-1}$, the frequency of deuterated palmitic acid. These labeled molecules are available in pure form, and the fatty acids may be incorporated synthetically into lipid constituents. A demonstration of this capability has been published, and is shown in Figure 5.10 (Holtom et al. 2001).

Other means for improving image contrast employ optical methods. We have the ability to collect a signal which is epi-directed, that substantially removes signal from bulk or isotropically distributed constituents and preferentially shows the subdiffraction limited size features within the focal

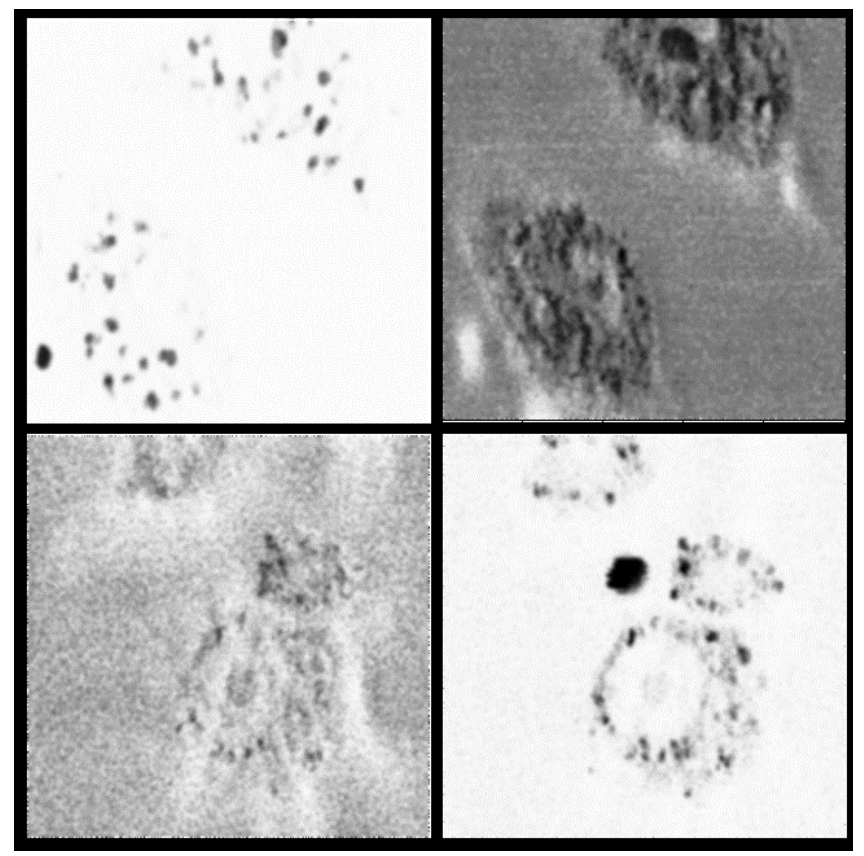

Figure 5.10. Illustration of the contrast provided with deuterated (D) labels in RAW264.7 cells (upper panels), compared with the normal hydrogen $(\mathrm{H})$ lipid mixture (lower panels). At a Raman frequency of $2120 \mathrm{~cm}^{-1}$ the D-lipid in vesicles is bright (upper left) and at $2850 \mathrm{~cm}^{-1}$ (upper right), only the more evenly distributed $\mathrm{H}$-containing species are present. The control experiment, using $\mathrm{H}$-lipids, has a very weak signal and low contrast at $2120 \mathrm{~cm}^{-1}$ (lower left), and the $\mathrm{H}$-vesicles appear brightly at $2850 \mathrm{~cm}^{-1}$ (lower right). Each image is $30 \mu \mathrm{m}$. 
volume. We have also implemented polarization methods to improve image contrast, using the fact that interfering electronic contributions to the image signal are always fully polarized, and the desirable vibrational signatures are usually depolarized

\section{Reference}

Holtom, G. R., B. D. Thrall, B. Y. Chin, H. S. Wiley, and S. D. Colson, Traffic 2, 781-788 (2001). 


\section{Ultrasensitive and Environmental Analysis}

This research area focuses on developing a suite of instrumentation for fast, accurate, highly sensitive, and discriminatory real-time analyses of chemical and biological natural or human-made agents. Such instruments are ideally suited to identify and quantify the many gases and pollutants, trace isotopic species, metabolic products in the breath, and chemical precursors and products from industrial processes. Some of these techniques can be developed for remote probing over long optical paths for remote interrogation of trace absorption features. Our infrared methods are supported by a state-of-the-art high-resolution molecular spectroscopy laboratory that is also used for studies of the structures and dynamics of molecular species important in contaminant chemistry, photochemistry, and atmospheric processes.

Characterization of particulate matter in the atmosphere represents a unique challenge. Several approaches are employed that, in combination, are designed to understand the evolving inventory of atmospheric particulate matter and how particulates are changed by reactions with gas phase species, photochemistry as well as condensation and evaporation processes. One approach uses new mass spectrometric techniques for aerosol analysis. This instrumentation is built around a laser desorption/ionization mass spectrometer with an asymmetric ion trap for simultaneous positive/negative ion detection. It provides real-time sampling and analysis of aerosol particles in a field-deployable unit more compact and less costly than current instruments.

In conjunction with this instrumentation, new devices are being developed and deployed to collect and preserve field samples for later automated analysis of individual particles in the laboratory. These same samples enable the chemical reactivity characterization of native aerosols for comparison to laboratory studies of the formation

and reactivity of model aerosol species. These studies are complemented by fundamental studies of the gas-phase chemistry of aerosol precursor molecules. 


\section{The PNNL Electron Microbeam}

G. Kimmel, M. S. Resat, B. Thrall,

L. Kathman, J. Miller, and D. Strom

Funded by the DOE Low Dose Radiation Research Program.

\section{General Description}

The PNNL electron microbeam uses energetic electrons to mimic radiation damage from gamma and $\mathrm{X}$-ray sources. It was designed such that high-energy electrons deposit energy in a pre-selected subset of cells so the unirradiated neighbors can easily be identified. By targeting individual cells with a highly spatially resolved dose, the biological responses of a single irradiated cell or a bystander can be studied. With this device, we study electron energies in the range of $20-80 \mathrm{keV}$ at various doses and dose rates.

Figure 6.1 is a schematic drawing of the electron microbeam, including the electron gun, vacuum system, sample stage and optical microscope. The electron gun (Kimball Physics EGH-8102/EGPS-8102) is housed in a small vacuum system and produces monoenergetic beams $\left(\Delta \mathrm{E}_{\text {init }} \sim 0.4 \mathrm{eV}\right)$ with energies ranging from $20 \mathrm{keV}$ to $80 \mathrm{keV}$. The electron beam current can be adjusted over a wide range $(\sim 1 \mathrm{pA}$ to $\sim 10 \mu \mathrm{A}$ ) and is measured with a Faraday cup. Rapid beam pulsing ( $\sim 10 \mathrm{~ns}$ resolution)

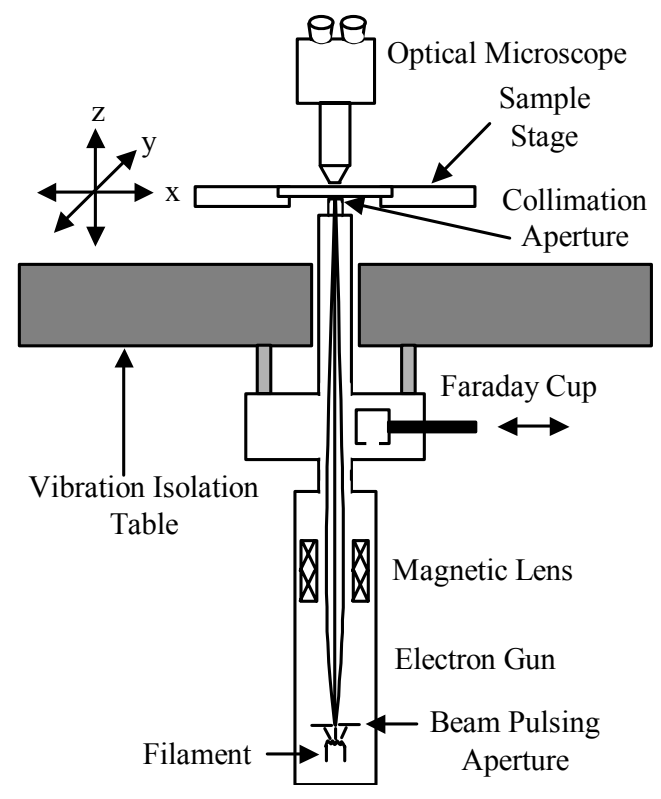

Figure 6.1. Electron microbeam. is achieved with a voltage pulse applied to the gun's suppression grid. A magnetic lens is used to focus the electron beam, typically to a diameter of $\sim 0.5 \mathrm{~mm}$. The final spatial resolution for the beam is achieved by passing the beam through an aperture (diameters $\sim 3 \mu \mathrm{m}-15 \mu \mathrm{m}$ ) fabricated in a thin metal foil typically $250 \mu \mathrm{m}$ thick tantalum. The apertures are fabricated by laser drilling (Resonetics Inc.) and are covered with a thin $(200-400 \mathrm{~nm})$ polyimide vacuum window (Luxel Corp.). The aperture is mounted on a small bellows assembly for adjusting its tilt relative to the beam.

The cell culture dishes consist of a Mylar film captured between two stainless steel plates with an o-ring seal. The Mylar film is placed directly on the electron microbeam aperture during irradiation (Figure 6.2). The thin, low-density films of polyimide and Mylar through which the microbeam is transported prior to irradiating the target are highly transparent to energetic electrons. The electron gun mounts on the underside of the vibration isolation table on which an upright optical microscope sits. The tilt assembly and aperture for the microbeam are inserted through the bottom of the microscope where the condenser would normally be, making the microbeam an integral part of the epifluorescence imaging system. The optical microscope, which has a focusing nosepiece, is positioned such that the electron beam aperture is centered in the field of view allowing for in situ monitoring of the irradiated area. The sample dishes are mounted on the $x-y-z$ stage of the optical microscope and the position can be set with sub-micron resolution. The dishes are re-locatable so samples can be targeted, irradiated, and removed for study at a later time, while still retaining information on the precise location of the

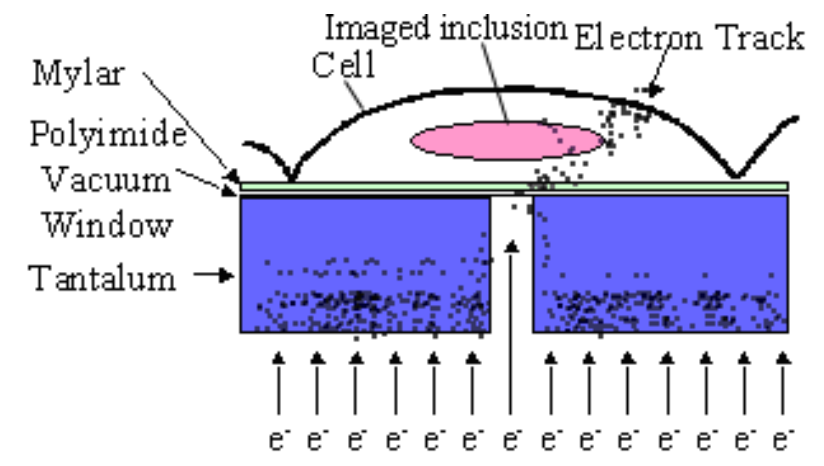

Figure 6.2. Schematic of electron microbeam sample interface. 
targeted dose. This feature allows for the possibility of combining the irradiator with other imaging analysis techniques using instruments in labs adjacent to the microbeam source. The cell irradiation area is contained within a temperature-controlled box that is maintained at $37^{\circ} \mathrm{C}$.

\section{Monte Carlo Simulations}

Based on the geometry of the PNNL electron microbeam, Monte Carlo simulations (Miller et al. 2000) suggest that $25-100 \mathrm{keV}$ electrons can irradiate individual mammalian cells in a confluent monolayer with minimal dose leakage to their neighbors. The simulations looked at energy deposition in various compartments (i.e., window, targeted cell, neighbors, or medium above cells). For ten thousand $25 \mathrm{keV}$ electron track simulations, no electrons were scattered to the spatial region occupied by neighbors of the target cell. The simulation also showed that varying the beam energy preferentially deposits energy in different regions of the targeted cell.

The spatial extent of the beam is largest at $50 \mathrm{keV}$, where the mean energy deposited in all the neighbors was 16 percent of that deposited in the target cell. This ratio was reduced to 5 percent at $90 \mathrm{keV}$. As the incident electron energy increases, both the penetrating power and the predominance of forward scattering increases. The former enhances lateral beam spread, while the latter diminishes it. A trade-off between these two effects produces the maximum spread at $50 \mathrm{keV}$.

The LET spectrum of the dose changes as a function of incident electron energy and can be obtained from the simulations. As we increase the beam energy, the lineal energy spectrum of energy deposited events approaches that of gamma rays. As the beam energy decreases, the event size distributions approach those expected for electron track ends (i.e., more like highLET radiation). By comparing the results at high and low beam energies, we can address different aspects of low-LET radiation. The ability to experimentally vary the LET distribution by changing the incident beam energy allows investigation of the relative biological importance of various parts of the energetic electron's track.

\section{Device Characterization}

We have used several approaches to characterize the operation of the electron microbeam. The electron beam current is measured with Faraday cups both before and after collimation. Dosimetric measurements are performed using GAFCHROMIC HD-810 dosimetry film. These films are suitable for beam profiling and dose mapping over a wide range of absorbed dose (Niroomand-Rad et al. 1998). Film calibrations were made at various doses using a ${ }^{60} \mathrm{Co}$ source. Figure 6.3 illustrates the spatial extent of the electron microbeam at two different energies, 50 and $80 \mathrm{keV}$. Figure $6.3 \mathrm{a}$ shows the beam profile at $50 \mathrm{keV}$ as measure on radiochromic film and imaged with a CCD camera. Figure $6.3 \mathrm{~b}$ shows the intensity profile along a line transecting the spot in Figure 6.3a. As was found in the theoretical work, we find the beam is broadened with increasing energy up to a point and then the predominance of forward scattering takes over. The full width at half maximum is approximately 25 and $10 \mu \mathrm{m}$ for 50 and $80 \mathrm{keV}$ respectively. The simulation results discussed above can obtain very specific information regarding the energy deposition by an electron in a cell. Experimentally, we can only measure an average over this distribution and, therefore, theoretical results can greatly enhance understanding of results for very low doses.

We have also used a sensitive, calibrated photodiode to characterize the spatial distribution of the electron microbeam in air. The spatial distribution of energy
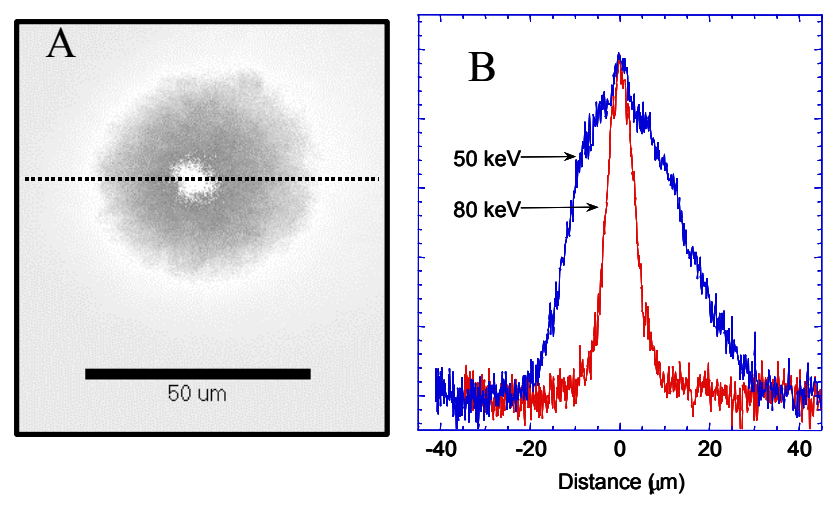

Figure 6.3. Spatial extent of the collimated electron beam as measured using radiochromic film. A: $50 \mathrm{keV}$ beam imaged with CCD camera using a 40X objective. B: Intensity profile along the line indicated in A. Similarly measured for $80 \mathrm{keV}$. 
deposition events of an electron track, $\mathbf{R}_{\mathrm{i}}$, in material, $i$, of density $\rho_{\mathrm{i}}$ is related to the track, $\mathbf{R}_{\mathrm{j}}$, in material, $j$, of density $\rho_{\mathrm{j}}$ by $\mathbf{R}_{\mathrm{i}} \rho_{\mathrm{i}}=\eta \mathbf{R}_{\mathrm{j}} \rho_{\mathrm{j}}$ where $\eta$ is a factor of order unity which depends on the materials, $i$ and $j$, but is nearly independent of the particle energy (Shultis and Faw 1996). Using this scaling relationship and the fact that the ratio of the density of air to water is $\sim 1200$, we can relate the beam measurements on the millimeter scale in air to the expected dose distribution in the target cells on the micron scale. In particular, the measurement in air corresponds to the dose distribution for a collimated point source in the target cell. Therefore, the total dose distribution in the target cell is given by the convolution of the collimated point source distribution and finite-sized exit aperture. This approach allows us to characterize the dosimetry of the electron microbeam in detail. We are collaborating with Prof. W. E. Wilson to compare our experimentally measured dose distributions with his Monte Carlo simulations of low-energy electron microdosimetry. We have also measured the X-ray background produced by the electron microbeam and found it to be negligible.

Figure 6.4 shows the intensity of a $50 \mathrm{keV}$ electron beam as a function of radius measured with the

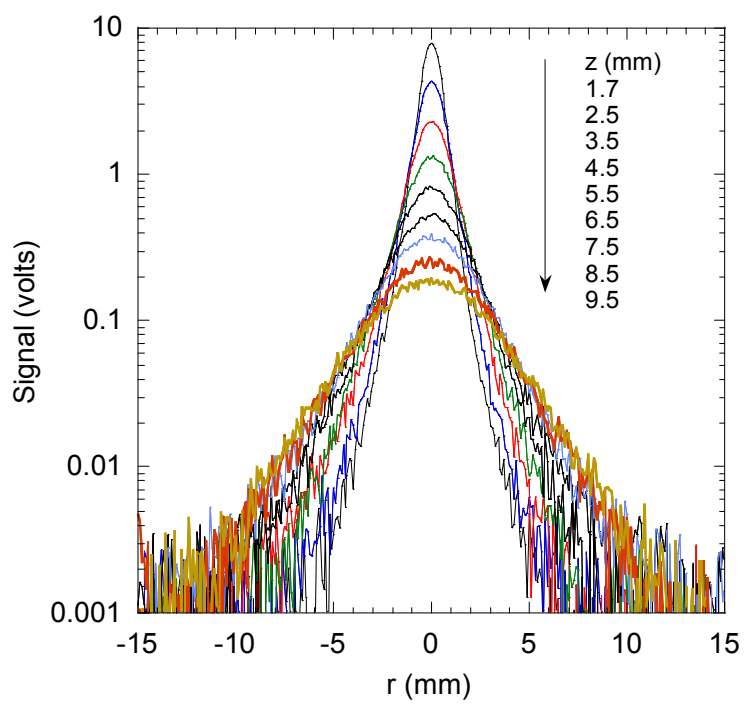

Figure 6.4. Beam intensity versus radius. The beam intensity versus radius in air for a $50 \mathrm{keV}$ beam was measured with a photodiode for several distances (z) of the photodiode above the aperture. photodiode at various distances from the exit aperture. At all heights, the electron microbeam has an approximately gaussian intensity distribution. Due to electron scattering, the peak intensity rapidly decreases and the beam width rapidly increases as the beam propagates through the air (or the biological target). At lower incident energies, the beam expands more rapidly due to enhanced electron-electron scattering.

Figure 6.5 shows the integrated beam intensity (integrated over a plane of constant height, $\mathrm{z}$ ) versus distance for a variety of different initial beam energies. The distance is plotted as the height, $\mathrm{z}$, normalized by the continuous stopping distance approximation (CSDA) range. (The CSDA range is related to the penetration depth of an energetic electron.) When distance is scaled by the CSDA range (or any other measure of the electron penetration depth such as the $\mathrm{x}_{90}$ range), the data fall on a universal curve. This observation is consistent with calculations of electron scattering kernels that show they are nearly independent of electron energy in the range from 25 to $100 \mathrm{keV}$ (Cross et al. 1992).

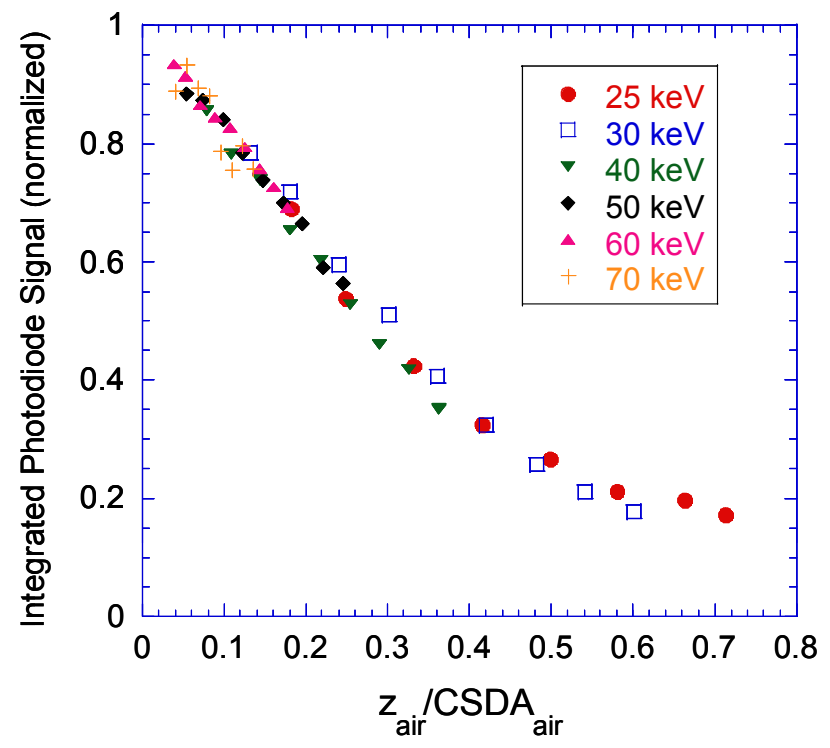

Figure 6.5. Integrated intensity versus height. For each energy, the height, $z_{\text {air }}$, is normalized by the CSDA range. For this energy range, the data fall on a universal curve when plotted in this manner. 


\section{Chromosome Damage Following Targeted and Non-Targeted Radiation Exposure}

Micronuclei are chromosomal fragments that are excluded from the primary cell nucleus at cell division. The micronuclei assay was used in various studies as a measure of chromosomal damage (Fenech 1997). To demonstrate the capabilities of the electron microbeam and to verify our operating conditions, we examined effects of ionizing radiation on human diploid fibroblasts, AG01522. These studies are patterned after work conducted at the Gray Laboratory using a micronuclei assay to study the effects of both targeted and non-targeted radiation upon the fraction of damaged cells produced (Belyakov et al. 1999, Belyakov et al. 2001, Prise et al. 1998). In the results presented below, we report the total fraction of damaged cells. We did not distinguish between micronuclei and apoptotic cells in the scoring of the preliminary data. From the Gray lab results, the percentage of damaged cells due to apoptosis exhibits a similar trend to that seen for micronuclei and at 3 days is about approximately 2 percent of the total fraction of damaged cells for low-LET X-ray data. Future studies will score apoptosis explicitly based on nuclear morphology.

\section{Non-Targeted Effects}

Dose Dependence at $30 \mathrm{keV}$ : Before conducting targeted irradiations, it is necessary to characterize the response of human diploid fibroblasts to "broad" beam electron radiation. Because our source has a finite size, these experiments are actually performed as irradiations of individual "spots" with sufficient overlap to obtain a uniform dose distribution over a broad area. Upon leaving the vacuum, the electrons are scattered in air and the lateral beam dimension is increased as the distance from the exit aperture is increased. Therefore, we perform these irradiations with an air gap of a few millimeters between the electron exit and the sample. Here the spot dimension is large compared to the step size on an $x-y$ translation stage. The $x-y$ translation stage is then stepped as the electron source is pulsed. The uniformity of the dose is verified using GAFCHROMIC film. Irradiations are done in $0.5 \mathrm{ml}$ of $20 \mathrm{mM}$ HEPES buffered growth medium at $37^{\circ} \mathrm{C}$. Bulk irradiations typically took
10 minutes per dish. Immediately following irradiation, cells are rinsed twice with fresh medium, $1 \mathrm{ml}$ is added and cells are incubated for 48 hours at $37^{\circ} \mathrm{C}$ and 5 percent $\mathrm{CO}_{2}$.

The fraction of damaged cells versus dose for an initial electron energy of $30 \mathrm{keV}$ is shown in

Figure 6.6. Complementary data obtained from ${ }^{60} \mathrm{Co}$ irradiations are also included. For the electron gun irradiation, we see a peak in the amount of chromosome damage at approximately $7 \mathrm{~Gy}$, closely paralleling the $5 \mathrm{~Gy}{ }^{60} \mathrm{Co}$ response. At higher doses, the percentage of damaged cells begins to fall off. Prise et al. (1998) observed a similar fall off for alpha particle irradiation and attributed it to a pronounced cell cycle delay. They found increased yields of damaged cells after 10 days incubation. Our preliminary results indicate a reduction in total cell number after higher dose exposures, consistent with the interpretation of Prise et al. (1998).

Initial Electron Energy Dependence: A unique feature of the microbeam device developed at PNNL is the ability to vary the energy of the incident electron. This feature allows us to examine different portions of the LET spectrum. Figure 6.7 shows the dependence on initial electron energy for non-targeted irradiation of human fibroblast at $3 \mathrm{~Gy}$. For the same total dose, there does not appear to be a strong

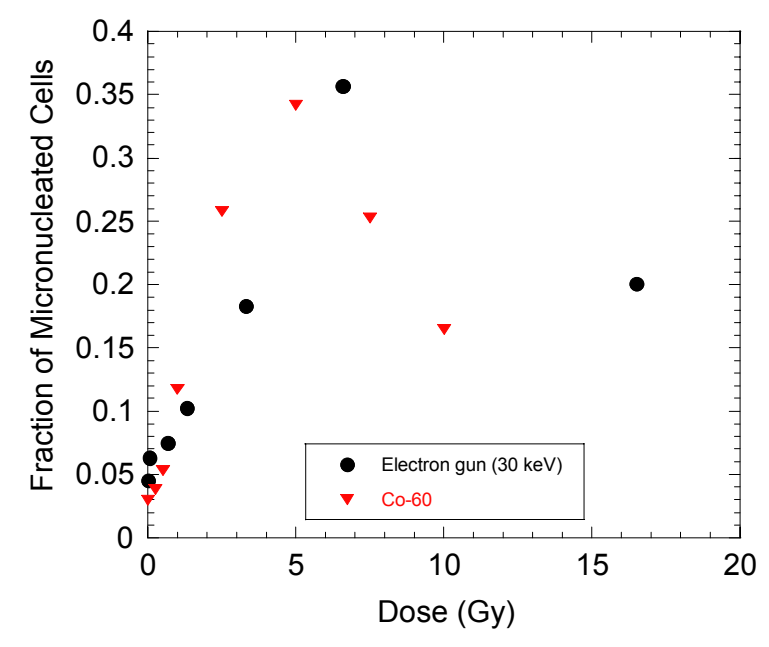

Figure 6.6. Non-targeted dose dependence upon the fraction of damaged cells at $30 \mathrm{kV}$. Also plotted is the dose dependence for ${ }^{60} \mathrm{Co}$ broad beam irradiation. 


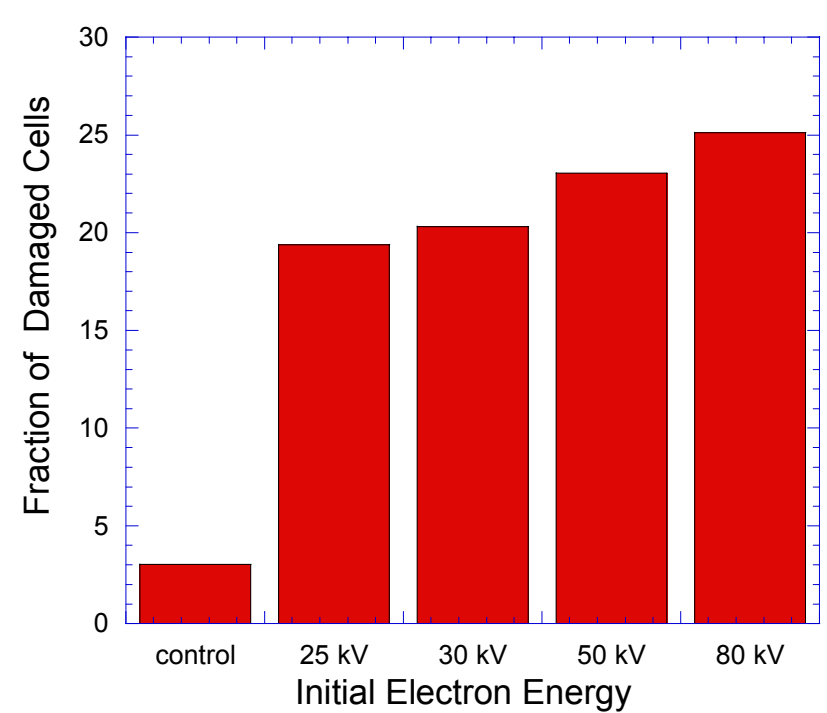

Figure 6.7. Initial electron energy dependence. Each bar represents the fraction of damaged cells produced at energies ranging from 25 to $80 \mathrm{kV}$.

dependence upon initial electron energy. However, we will need to obtain the complete dose response at each incident energy (as in Figure 6.6 for $E_{i}=30 \mathrm{keV}$ ) before this somewhat surprising result can be fully understood.

\section{Targeted Effects}

Figure 6.8 shows the capability of the gun to deliver a targeted dose to single cells. Cells were plated at a density of approximately 5 cells $/ \mathrm{mm}^{2}(\sim 100$ cells total) and either 25 or 50 cells were irradiated with targeted $30 \mathrm{keV}$ electrons, in contact with the gun.

We observed an increase in the fraction of damaged cells as we increased the number of cells targeted. These initial experiments were done at a high dose ( $>10$ Gy). Therefore, the intent is simply to demonstrate the ability of the gun to cause cellular damage when used in the targeting mode. In the experiment reported here, we targeted a fixed number of cells. Ongoing experiments are being conducted with optimized microbeam settings and where the precise number of cells on a plate is first obtained by counting on a phase contrast microscope. Variability in the plating of the fibroblasts on Mylar makes the latter step necessary to ensure we are targeting an exact fraction of cells in the population.

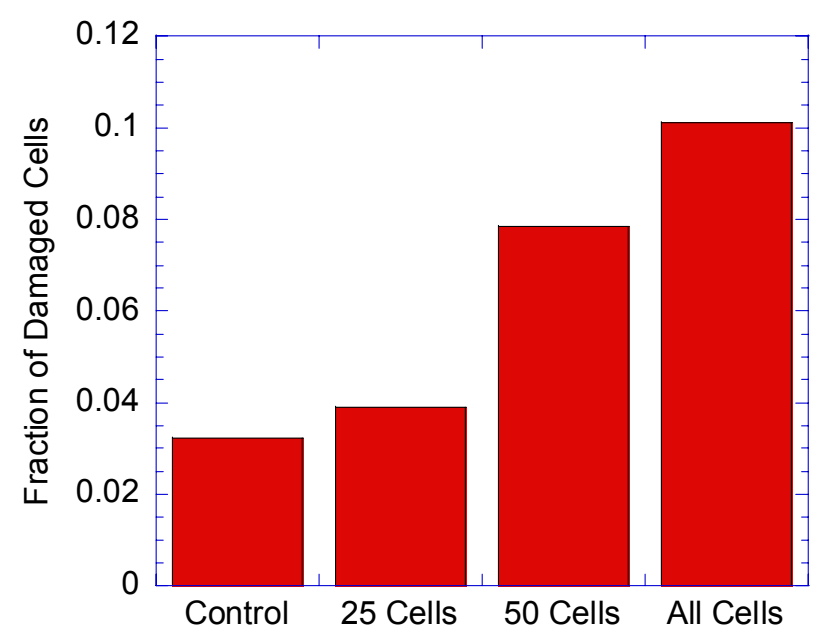

Figure 6.8. The effect of targeted population dosing on the fraction of damaged cells. The data obtained where all cells were irradiated was performed as a non-targeted broad beam irradiation discussed in the text.

\section{References}

Belyakov, O. V., K. R. Trott, B. D. Michael. International Journal of Radiation Biology 75(8), 985-993 (1999).

Belyakov, O. V., A. M. Malcolmson, M. Folkard, K. M. Prise, and B. D. Michael. British Journal of Cancer 84(5), 674-679 (2001).

Cross, W. G, N. O. Freedman, and P. Y. Wong. Tables of Beta-Ray Dose Distributions. Chalk River Laboratories, Chalk River, Ontario, Canada, (1992).

Fenech, M. Mutation Research-Genetic Toxicology and Environmental Mutagenesis. 392(1-2), 11-18 (1997).

Niroomand-Rad, A., C. R. Blackwell, B. M. Coursey, K. P. Gall, J. M. Galvin, W. L. McLaughlin, A. S. Meigooni, R. Nath, J. E. Rodgers, and C. G. Soares. 1998. "Radiochromic Film Dosimetry: Recommendation of AAPM Radiation Therapy Committee Task Group 55, Medical Physics 25(11): 2093-2115.

Prise, K. M., O. V. Belyakov, M. Folkard, and B. D. Michael. 1998. "Studies of Bystander Effects in Human Fibroblasts Using a Charged Particle microbeam. International Journal of Radiation Biology 74:793-798. 


\section{Chemistry of Atmospheric Aerosols}

\author{
A. Laskin, M. J. Iedema, J. P. Cowin,
}

R. S. Disselkamp, C. M. Berkowitz, and

J. S. Young

Supported by the US Department of Transportation, DOE Office of Basic Energy Sciences, and EMSL Operations.

\section{Time-Resolved Field Studies of Particles}

Traditional field studies of atmospheric pollution gather much data on time-resolved concentrations of trace molecules, but comparably little data on the time-resolved chemical makeup of atmospheric particles believed to influence that pollution. Atmospheric particulates comprise a complex mix of specific minerals, organics, etc. To best understand them in the field, time-resolved speciation of individual particles is essential. We constructed a Time Resolved Aerosol Collector (TRAC) to address these needs. The field device is compact and inexpensive, and permits much more extensive aerosol characterization than would any field-based analysis that collected aerosols and returned them to the lab. Laboratory characterization includes

1. automated single-particle analysis of elemental composition via scanning electron microscopy (SEM) and energy-dispersed $\mathrm{x}$-ray analysis (EDX)

2. time-of-flight secondary ion mass spectrometry (TOF-SIMS) to probe the inorganic and organic composition of both the aerosol surface and bulk with nanometer depth resolution, probing any organic overlayers.

Additionally, Temperature Programmed Desorption (TPD) performed in the TOF-SIMS instrument will characterize the behavior of reactive gases on the collected particles, as well as probe desorption kinetics of semi-volatile components in the aerosols. The SEM/EDX is the main tool, and as automated, can analyzed over 20,000 particles per day, for all elements of a mass $>$ boron, and for particles 0.2 micron in size or larger.

We have also developed a very small (button sized) and inexpensive passive monitor for personal exposure determination, the Tiny Air Quality monitor (TAQ). Somewhat larger and more sensitive active collectors are also being created. These allow us to examine epidemiological links in populations between specific particulate species (e.g., $\mathrm{NH}_{4} \mathrm{HSO}_{4}$, diesel soot, wood smoke, etc.) and health effects (including possible mechanisms for those health effects).

Field Study-Houston, 2000: In the fall of 2000, we used our automated sampler, as part of a group of a national team of researchers, in a month-long study of Houston air pollution. The formation, composition, and day-night cycles of ozone and particulate matter were studied, as well as how these pollutants are affected by weather. We have around 3000 samples taken at 10 minute intervals for almost all of the 30-day study (each sample contains thousands of aerosol particles). Figure 6.9 shows analytical results for about 100 particles, from just one sample. We can select from the complex mix of particles any subgroup, to follow versus time. Looking just sea-salt derived particles (leftmost in Figure 6.9) we see that many are depleted in $\mathrm{Cl}$ and are richer in $\mathrm{O}$ and $\mathrm{N}$ than fresh sea salt should be. The sea-salt particles are reacting with trace $\mathrm{HNO}_{3}$ in the air, during their 70-mile trek from the ocean, leaving $\mathrm{NaNO}_{3}$ particles and gaseous $\mathrm{HCl}$. We can follow this stochiometrically, as shown in Figure 6.10, versus time (one 24 period shown). Much other information can be extracted from the data such as secondary organic material growth on mineral particles or heterogeneous conversion of $\mathrm{SO}_{2}$ to $\mathrm{H}_{2} \mathrm{SO}_{4}$. 
Each vertical line is a bar graph of an individual particle's composition

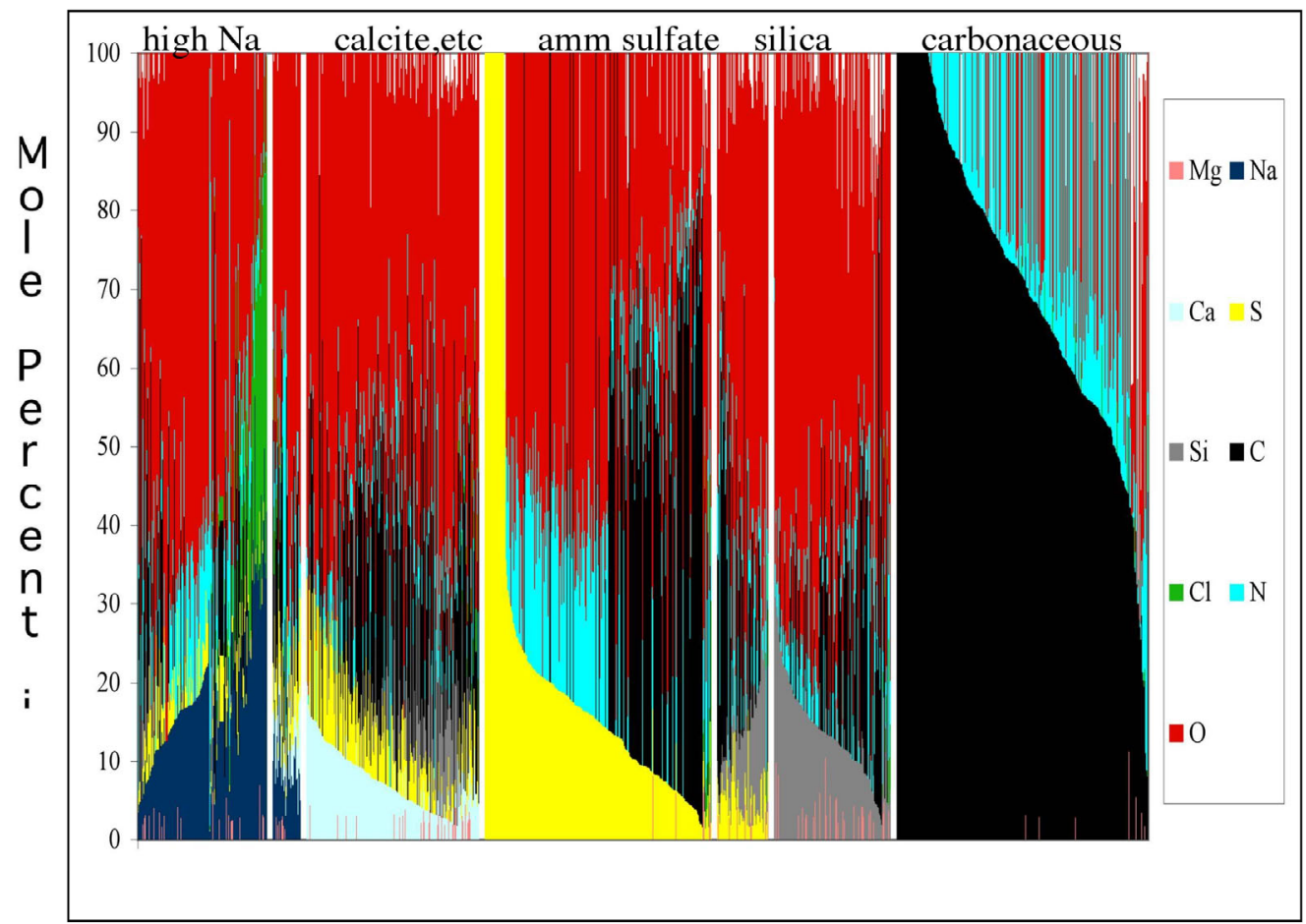

\section{All particles $<0.7$ micron, 8/17/00 11:08 to 11:18 AM}

Figure 6.9. Individual particle compositions. Each vertical, multicolored line represents a single particle, form one sample, from Houston. The colors represent the elemental composition of the particles. The particles have been sorted into several groups.

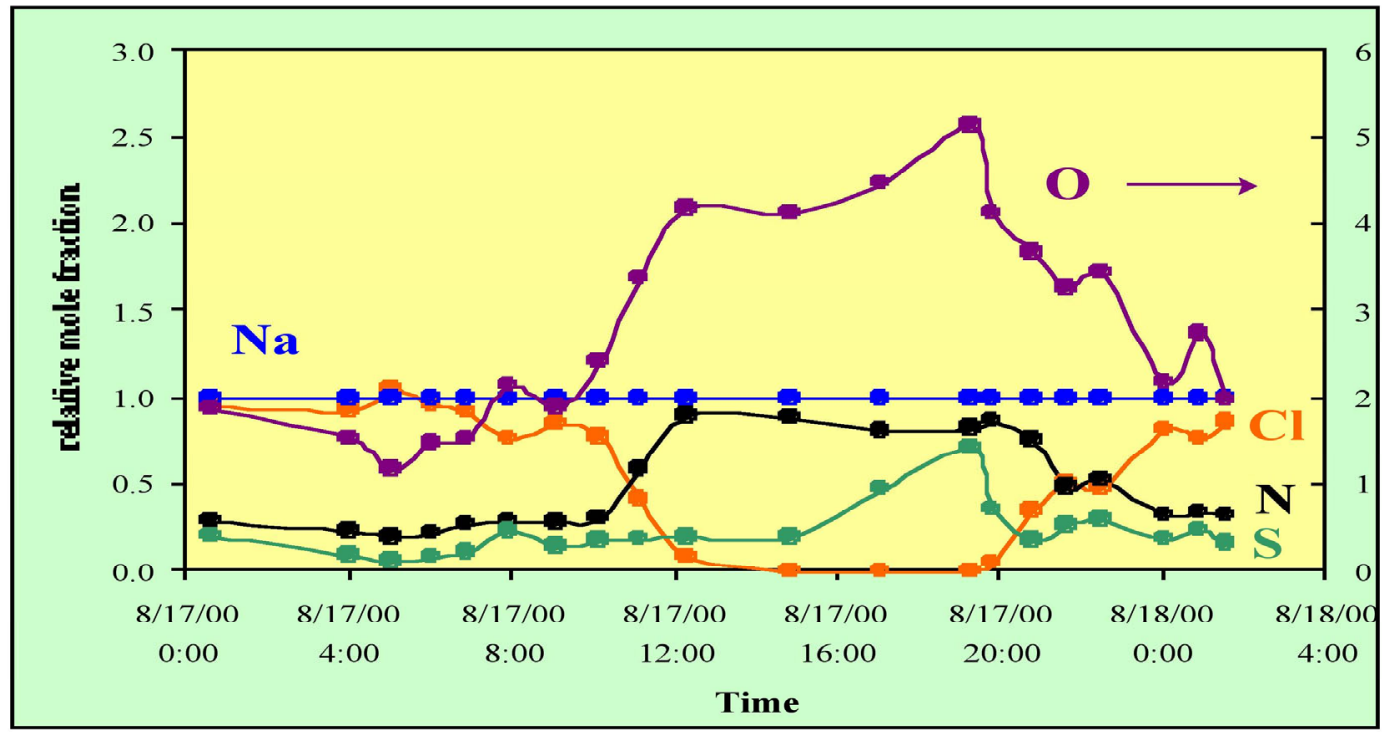

Figure 6.10. Sea salt derived particles. In Houston, Texas, the sea salt seldom gets there from the ocean. The composition of just this subclass is shown versus time, for one 24 hour period. 


\section{Laboratory Measurement of Water Nucleation Using a Laminar Flow Tube Reactor}

\author{
V. B. Mikheev, ${ }^{(a)}$ P. M. Irving, ${ }^{(a)}$ N. S. Laulainen ${ }^{(b)}$ \\ S. E. Barlow, ${ }^{(b)}$ and V. V. Pervukhin ${ }^{(c)}$
}

This research was supported by the DOE Office of Biological and Environmental Research.

(a) Innova Tek, Inc, Richland, Washington

(b) Pacific Northwest National Laboratory

(c) Institute of Inorganic Chemistry of SB RAS, Novosibirsk, Russia.

\section{Introduction}

When pure water vapor is mixed with an inert gas such as pure helium, $\mathrm{O}_{2}, \mathrm{~N}_{2}$, etc., and the gas cooled, the water will not spontaneously condense into droplets until the relative humidity is several times the equilibrium saturation value. This reluctance to cross the phase boundary is completely analogous to the more familiar phenomena of super heating, or cooling. Instead, normally in nature, trace "impurities" are said to "nucleate" the phase transitions. Thus, in the atmosphere, we never find more than 100 percent relative humidity. (By contrast, an undergraduate chemistry instructor always warns beginners to add boiling chips before attempting to boil a clean solution.)

One might believe that such a ubiquitous process as nucleation would be well studied and understood. In fact it is not. This is because a "super" state is metastable and almost any perturbation can initiate the phase transition. In particular, gas-to-condensedphase transitions are very sensitive to impurities, turbulence and temperature. The large response to perturbations has been known for more than a century and forms the basis for very sensitive detection schemes such as the Wilson cloud chamber of highenergy physics.

Ideally, a study of nucleation would begin with an understanding of the behavior of pure substances and then move to more complex systems. (In the language of homogeneous nucleation, the nucleation of a single component is called "unary," if two different substances, say water and sulfur trioxide are involve it is called "binary.") In so far as pure substances are concerned, the nucleation and condensation of water is perhaps of greatest interest and importance owning to its unique role on the earth's surface, in its atmosphere, and in biological systems. In spite of repeated attempts over the years, it is only very recently that reliable measurements of water nucleation have become available (Wölk and Strey 2001).

Ultimately we would like to investigate binary (or higher order) nucleation with water because of its environmental importance. However the techniques which produced the best water nucleation results are unsuited to the study of binary systems. To circumvent these limitations, we designed, built, and tested a Laminar Flow Tube Reactor (LFTR).

However, before we can make definitive statements about complex systems, we needed to validate our approach. We did this by remeasuring pure water nucleation. We found excellent agreement with Wölk and Strey (2001) for the temperature ranges where our data overlapped. We were however able to extend the measurements to even lower temperatures where we found significant difference between our results and those expected by classical nucleation theory.

\section{Experimental}

To conduct these experiments, we designed, built, and tested the new Laminar Flow Tube Reactor (LFTR) illustrated schematically in Figure 6.11. The LFTR features very heavy walled aluminum tubes through which we bored channels for coolant to circulate. Adjacent channels were connected via headers on each end of the main tube and these headers were sealed with ultra high vacuum (UHV) compatible indium gasket wire. In fact we found it essential to use UHV throughout the design to reach adequate levels of purity for nucleation measurements.

The LFTR has two main sections - the saturator and the condenser. The ultra high purity (UHP) helium carrier gas flows from one section to the other through a $1 \mathrm{~mm}$ gap that is defined by a Teflon gasket. Because of the massive construction and the inherently high thermal conductivity of aluminum, only minor thermal gradients exist along the respective surfaces of the central flow tube in the vicinity 


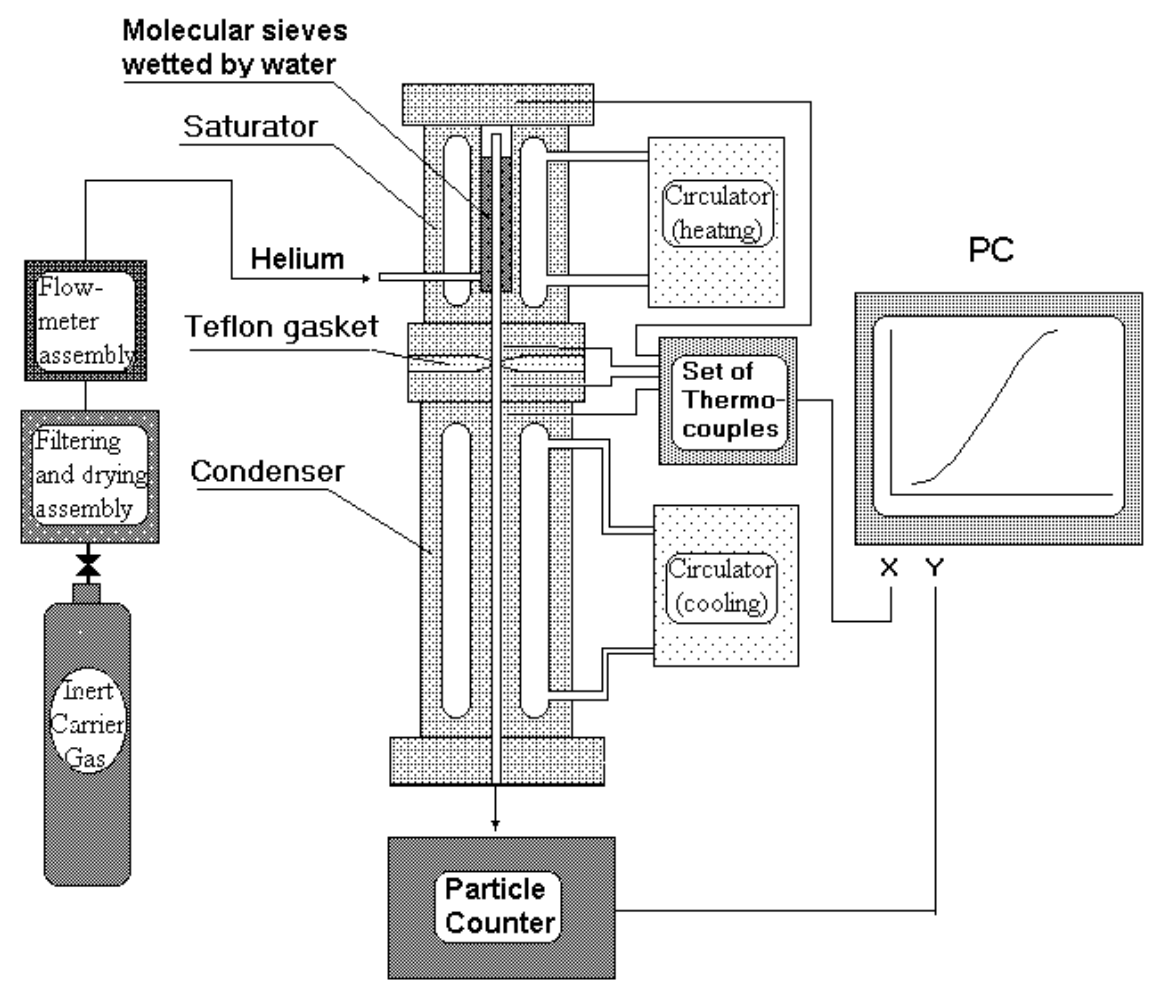

Figure 6.11. General schematic of experimental setup.

of the insulator. Temperature was stabilized in each section by its own heater/chiller bath. These temperatures were generally found to be stable to \pm 0.05 $\mathrm{C}$ or better.

In operation, helium buffer is introduced to the saturator as shown in Figure 6.11. It first passes through a porous medium that was saturated with liquid water and becomes saturated itself. (We went to considerable lengths to insure that it indeed was saturated). Upon entering the condenser, the gas begins cooling immediately and becomes supersaturated. When a critical temperature is reached (Becker and Doring 1935; Zeldovich 1942) nucleation begins, its rate, $\mathrm{J}$, being dependent on the supersaturation ratio, $\mathrm{S}$. The freshly nucleated particles continue down the stream and grow. Eventually they reach the optical particle counter and are measured.

Proper measurement requires that nucleation occur predominately near the central axis of the flow tube. This requires detailed modeling of the gas flow and its thermal evolution. We find that by carefully controlling the flow, we can indeed force nucleation to occur on axis as opposed to near the walls. Of course, ice formation and build-up are continual problems and after each measurement the system must be dried before the next measurement.

\section{Results}

Figure 6.12 is shows two log-log lots of nucleation rate, J, versus super-saturation, $\mathrm{S}$ for various temperatures. Figure 6.12a compares our results with classical nucleation theory predictions. At higher temperatures we have excellent agreement, but as the temperature falls our results are three orders of magnitude smaller that the theory predicts. (One order of magnitude is generally considered good agreement for nucleation measurements.) It is very difficult to see how this could be an experimental problems - most problems cause the measured rate to increase. The source of the problem probably lies in the relatively simplistic extrapolation of bulk liquid properties down to the region of 15 to 20 molecules that goes into the calculations of the classical theory.

Recent theoretical developments that include a new paradigm for thinking about nucleation and allow the inclusion of realistic intermolecular forces may help to resolve these discrepancies (Kathmann et al. 2002). 


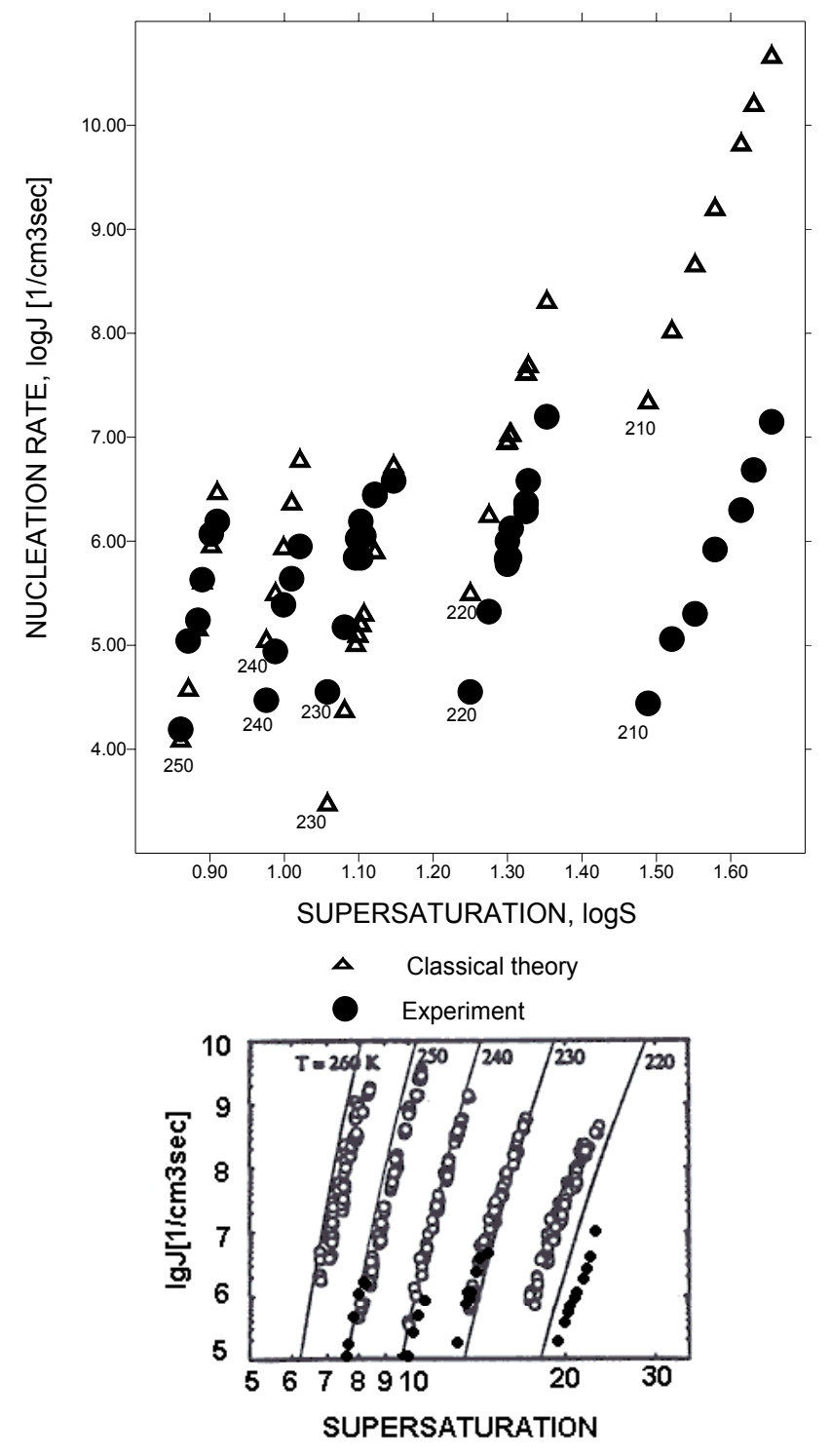

Figure 6.12. Nucleation of water in He. (a) Experimental results (black circles) of nucleation rate $J$ dependence on supersaturation $S$ and theoretical predictions (triangles) made using classical nucleation theory (Becker and Doring 1935; Zeldovich 1942) (b) A comparison of our data (black circles) with Wölk and Strey (2001) (open circles) and with the classical theory (Becker and Doring 1935; Zeldovich 1942) predictions. Nucleation temperatures (in K) are indicated at the bottom (top) of each data set.

\section{Acknowledgements}

This work has been supported by the Atmospheric Chemistry Program of the U.S. Department of Energy. This research was performed in the William R. Wiley Environmental Molecular Sciences Laboratory, a national scientific user facility sponsored by the
Department of Energy's Office of Biological and Environmental Research and located at Pacific Northwest National Laboratory. Pacific Northwest National Laboratory is operated for the DOE by Battelle Memorial Institute. It has also been supported by InnovaTek, Inc., Richland, Washington.

\section{References}

Becker, R. and W. Doring, Ann. Phys. (Leipzig) 26, 719 (1935).

Kathmann, S. M., G. K. Schenter, and B. C. Garrett, J. Chem. Phys. 116, 5046, (2002).

Wölk, J. and R. Strey, J. Phys. Chem. B 105, 11683 (2001)

Zeldovich, Ya. B., Zh. Eksp. Teor. Fiz. 12, 525 (1942).

\section{High Resolution Infrared Spectroscopy Facility}

\section{T. A. Blake, R. S. Disselkamp, T. J. Johnson, J. F. Kelly, R. L Sams, and S. W. Sharpe \\ This research was supported by EMSL Operations, LDRD funding, and the DOE Office of Nuclear Non-Proliferation.}

\section{Introduction}

Infrared spectroscopy is one of several powerful techniques that the physical chemist can call upon for studying the behavior of matter on an atomic scale. In addition, infrared spectroscopy forms the basis for a suite of analytical techniques that are highly specific, extremely sensitive, and can be directly applied to a number of monitoring problems. Brief descriptions of several ongoing projects in both basic and applied research are summarized below for the PNNL Infrared Spectroscopy group.

\section{Tools of the Trade}

High Resolution Fourier Transform Infrared Spectrometer: A well maintained Bruker 120HR Fourier transform infrared (FTIR) spectrometer is the workhorse instrument for much of our basic research. This system is based on a double pass, 3-meter Michelson interferometer capable of $0.0015 \mathrm{~cm}^{-1}$ spectral resolution. Spectral coverage includes the ultraviolet $\left(30,000 \mathrm{~cm}^{-1}\right)$ through the millimeter $\left(10 \mathrm{~cm}^{-1}\right)$ region. The $120 \mathrm{HR}$ can be interfaced to a 
number of commercial and custom sampling systems including a 40-meter White cell and a soon to be completed 200-meter temperature controlled White cell. In addition, gas samples may be introduced to a 20-cm single pass, temperature controlled cell with precision pressure measurement capabilities (Baratron transducers, 0.12 percent full scale accuracy).

A Cool Source of Molecules: High resolution gas phase infrared spectroscopic techniques have traditionally been plagued by a number of problems including spectral congestion, Doppler broadening, and pressure broadening. Spectral congestion is related to the number of quantum states populated at a given temperature and dictated by Maxwell-

Boltzmann statistics. Doppler broadening is related to temperature through the kinetic energy relationship but also involves the random three-dimensional motion of the gas molecules. Pressure broadening is related to temperature through the collision frequency of the molecules which, in turn depends on the density and velocity of the sample. By cooling and reducing the pressure of a gas sample through supersonic adiabatic expansion these three broadening effects may be substantially reduced.

Supersonic expansion and subsequent rotational cooling is accomplished by expanding a gas sample through a slit orifice that measures $12 \mathrm{~cm}$ in length by $50 \mu \mathrm{m}$ wide. The ensuing ribbon of gas expands at supersonic velocities and in the process molecules entrained in this ribbon are cooled to a few degrees above absolute zero. In addition, the random three-dimensional motion of the gas molecules are converted to a two-dimensional flow with only a small velocity component in the plane of expansion but perpendicular to the mass flow. If infrared light is used to interrogate the gas molecules, through the plane of expansion, spectral congestion, Doppler broadening and pressure broadening are reduced significantly.

Recently, we have succeeded in interfacing our Bruker 120HR FTIR with a continuous slit expansion source. The PNNL FTIRS-beam machine is capable of recording high resolution $\left(\Delta v \geq 0.0015 \mathrm{~cm}^{-1}\right)$ spectra at rotational temperatures of $15 \mathrm{~K}$ anywhere in the near- to far-infrared spectral region (see Figure 6.13).

\section{Basic Research}

Much of the basic research that we do is through external collaborations with academic and government institutions. This allows us to share our unique high resolution infrared capabilities with other experts in the field.

Sulfur Trioxide: One of these ongoing collaborations includes a detailed study of the sulfur trioxide molecule. In an effort to improve the quality of the spectroscopic and force field constants of the sulfur trioxide molecule, we have entered into a collaboration with researchers at Oregon State University to record and analyze the rotationally resolved infrared spectra of this molecule. We have recorded all of the infrared fundamental, overtone, and combination bands from 400 to $3000 \mathrm{~cm}^{-1}$ of the four isotopic modifications ${ }^{32} \mathrm{~S}^{16} \mathrm{O}_{3},{ }^{32} \mathrm{~S}^{18} \mathrm{O}_{3},{ }^{34} \mathrm{~S}^{16} \mathrm{O}_{3},{ }^{34} \mathrm{~S}^{18} \mathrm{O}_{3}$. These gas phase spectra were recorded in a room temperature cell using our Fourier transform spectrometer. The resolution at which the spectra were recorded $\left(0.002 \mathrm{~cm}^{-1}\right)$ is a factor of two better than the studies of this molecule performed in the 1970s and 1980s (Kaldor and Maki 1973; Ortigoso et al. 1989). As a

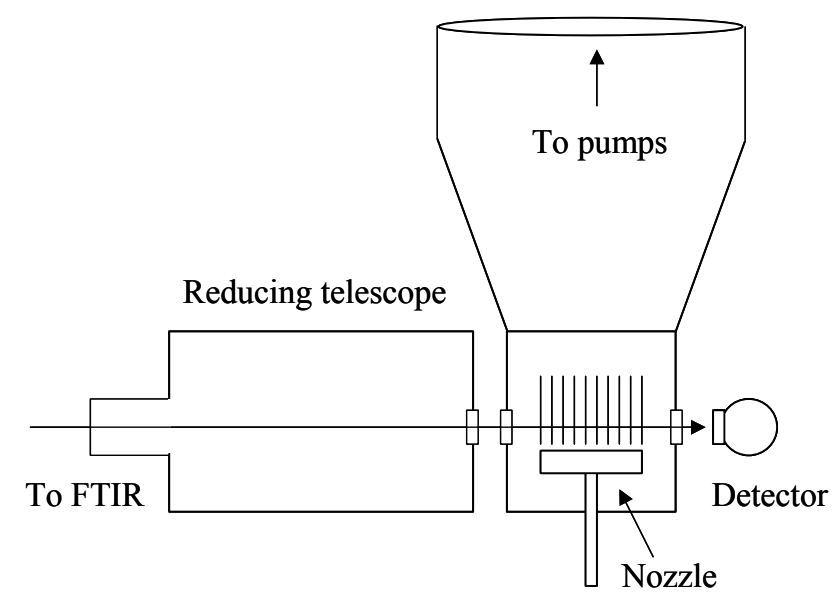

Figure 6.13. Layout of FTIR spectrometer-supersonic nozzle source. Box at left contains beam reducing optics between FTIR and expansion chamber. Expansion chamber contains supersonic nozzle and multi-pass (White cell) optics for increased optical path length (not shown in figure). Pumping system consists of 4 roots blowers backed by a large mechanical pump. Pumping speed is approximately 6,000 liter/sec at pressures up to 10 torr, giving the pumping system superb throughput. 
result, we have been able to not only fit a set of ground state rotational constants from the complete data set, but also, for the first time, we have been able to see the effects of Coriolis coupling perturbations on the rotational structure of the spectra. These effects can now be taken into account when fitting the upper state vibrational energy levels. Data and analysis of the $v_{2}, v_{4}, v_{3}$, and $2 v_{3}$ infrared bands of ${ }^{32} \mathrm{~S}^{16} \mathrm{O}_{3}$ have recently been presented in the literature (Maki et al. 2001) along with preliminary results for the Raman active $v_{1}$ bands of all the aforementioned isotopomers (Chrysostom et al. 2001).

The Raman spectrum of the $v_{1}$ band of ${ }^{32} \mathrm{~S}^{16} \mathrm{O}_{3}$ is shown in Figure 6.14 (thin trace) and was recorded using a Coherent Anti-Stokes Raman Spectrometer (CARS) at Oregon State University. The band shows a significant amount of perturbation, particularly evidenced by the large gap at $1066.5 \mathrm{~cm}^{-1}$. To understand the nature of these perturbations it was necessary to obtain accurate spectroscopic constants of the nearby perturbing states. This was done by recording and assigning the hot band transitions associated with the perturbing states, namely $2 v_{2}, 2 v_{4}(1=0)$, and $2 v_{4}$ $(1=2), v_{2}+v_{4}$, arising from the low frequency fundamentals $v_{2}$ and $v_{4}$ (see Figure 6.15). A detailed analysis of the perturbations shows that the $v_{1}$ band of ${ }^{32} \mathrm{~S}^{16} \mathrm{O}_{3}$ is perturbed by Fermi resonances from the $2 v_{2}$ and $2 v_{4}$ states, but these states in turn are perturbed by the close lying $2 v_{4}(1=2)$ and $v_{2}+v_{4}(l=1)$ states via Coriolis couplings (see Figure 6.16). The CARS spectrum can now be calculated from first principles using the spectroscopic constants determined in this study (see the thick trace in Figure 6.14). A manuscript detailing this work is in preparation (Barber et al. manuscript in preparation).

Nitric acid: Nitric acid plays an important role in the NOx chemistry of the earth's atmosphere and the ability to accurately quantify its concentration in field campaigns has become a major goal of atmospheric chemists. Typical tropospheric concentrations are on the order of $100 \mathrm{ppt}$ to $1 \mathrm{ppb}$ in remote to rural areas and Fourier transform infrared spectroscopy or tunable infrared diode laser spectroscopy is used to make these measurements (Finlayson-Pitts 2000). Consistent laboratory data relating observed infrared line strengths to concentration does not exist (Giver et al. 1984; Goldman et al. 1998), so we have entered

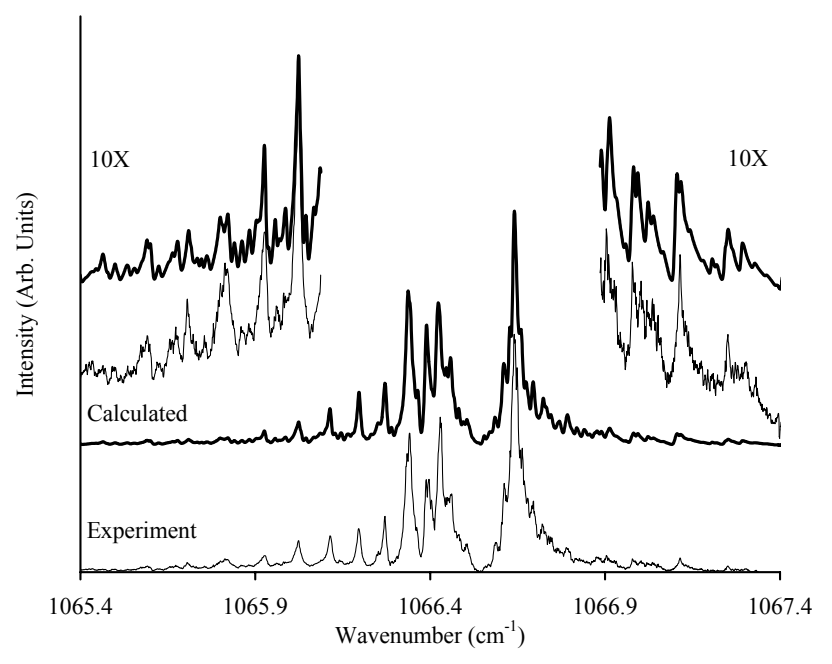

Figure 6.14. Raman spectrum of the $v_{1}$ band of ${ }^{32} \mathrm{~S}^{16} \mathrm{O}_{3}$. The spectrum was recorded using a CARS spectrometer at Oregon State University. Only the Q-branch is observed. The thin line trace is the experimental result, which shows a strong perturbation of the band. The heavy line trace is the calculated spectrum.

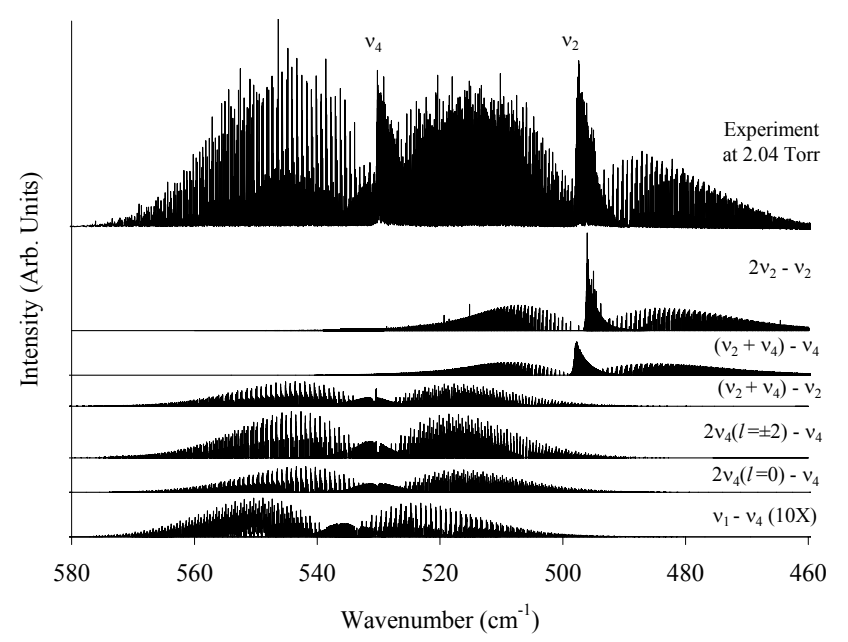

Figure 6.15. Infrared spectrum of the $v_{4}$ and $v_{2}$ region of ${ }^{32} \mathrm{~S}^{16} \mathrm{O}_{3}$ (top trace). The spectrum was recorded at PNNL using a high resolution Fourier transform infrared spectrometer. The lower traces show the hot bands separated from the fundamental spectrum. Assignment of the hot band spectra was necessary to understand the perturbations of the $v_{1}$ band.

absolute infrared cross sections, line shift, and broadening parameters for anhydrous nitric acid. Nitric acid is a notoriously sticky molecule and measuring its concentration in a sample cell is difficult, but with some care in the selection of cell 


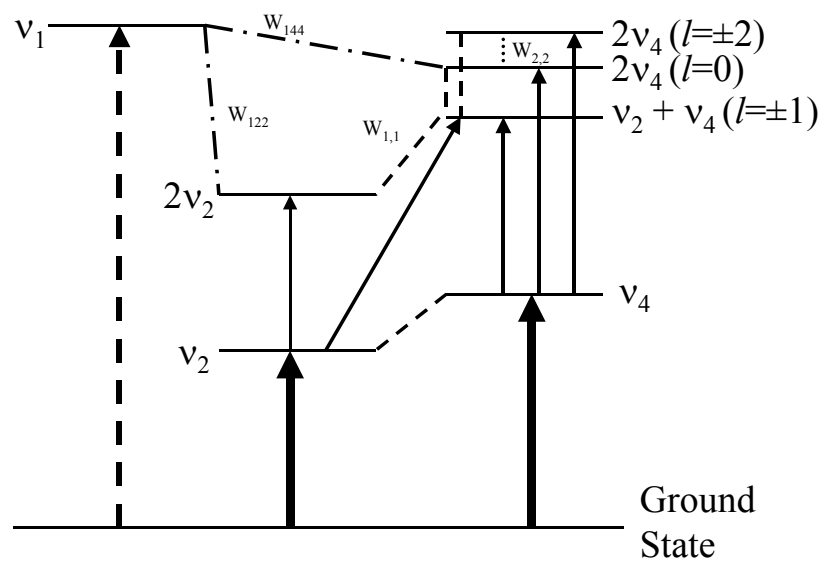

Figure 6.16. Energy level diagram showing the perturbations affecting the $v_{1}$ fundamental. The $W_{122}$ and $W_{144}$ are Fermi resonances between $v_{1}$ and $2 v_{2}$ and $2 v_{4} . W_{1,1}$ is a Coriolis interaction between states with different vibrational quantum numbers. $W_{2,2}$ is a Coriolis interaction within a vibrational state.

and window materials and experimental technique that includes flowing the compound through the absorption cell, we have been able to measure absolute absorption cross sections for individual ro-vibrational transitions for several fundamental vibrational bands using our high resolution Fourier transform spectrometer. Analysis of the data is ongoing. A spectrum of the $v_{2}$ band of anhydrous $\mathrm{HNO}_{3}$ is shown in Figure 6.17.

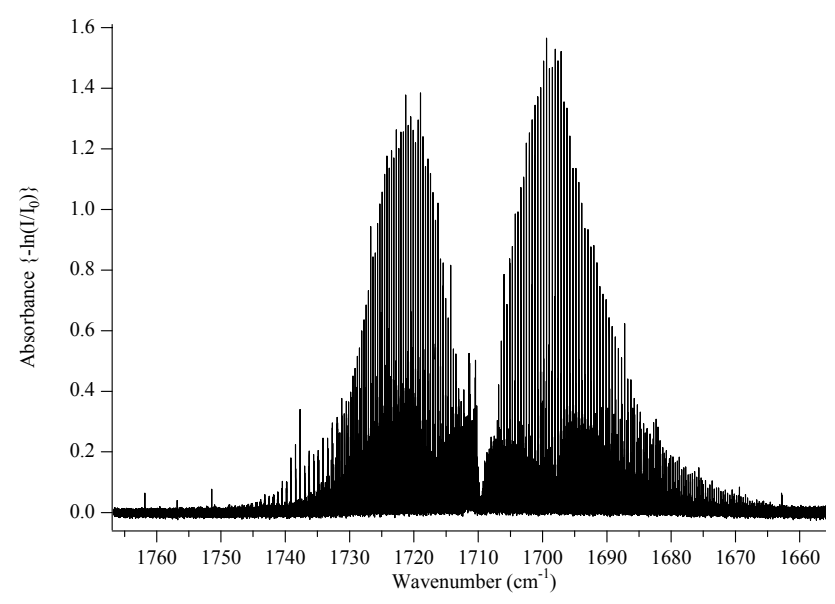

Figure 6.17. $v_{2}$ band of anhydrous $\mathrm{HNO}_{3}$. The spectrum was recorded using a 0.244 torr sample in a $10 \mathrm{~cm}$ cell, which was constructed from gold-coated stainless steel and was capped with $\mathrm{BaF}_{2}$ windows. Spectral resolution was $0.0025 \mathrm{~cm}^{-1}$. Sixty-four scans were co-added.
Perfluorocyclobutane: For many molecules, analysis of rotationally resolved infrared spectra is not possible due to the high density of states populated at room temperature. One such molecule is perfluorocyclobutane. For perfluorocyclobutane, high resolution infrared spectroscopy at room temperature provides no insight into the nature of the molecule's vibrational band structure. In fact, band assignments and the structure of this molecule have been debated for more than thirty years (Fisher et al. 1997). We have recently recorded jet cooled spectra of the vibrational bands of this molecule that show that previous band assignments are, in fact, erroneous. The spectra are recorded by crossing the focused light from a high resolution Fourier transform infrared spectrometer with the supersonic expansion of a mixture of 10 percent $c-\mathrm{C}_{4} \mathrm{~F}_{8}$ in helium. Figure 6.13 shows a schematic of the interface of the spectrometer and the supersonic expansion chamber. Figure 6.18 shows the $v_{13}$ fundamental vibrational band recorded in this manner. The rotational temperature of the jet is approximately $15 \mathrm{~K}$ and the vibrational temperature is estimated to be about $170 \mathrm{~K}$. The rotational structure of the parallel band centered at $1240.34858(4) \mathrm{cm}^{-1}$

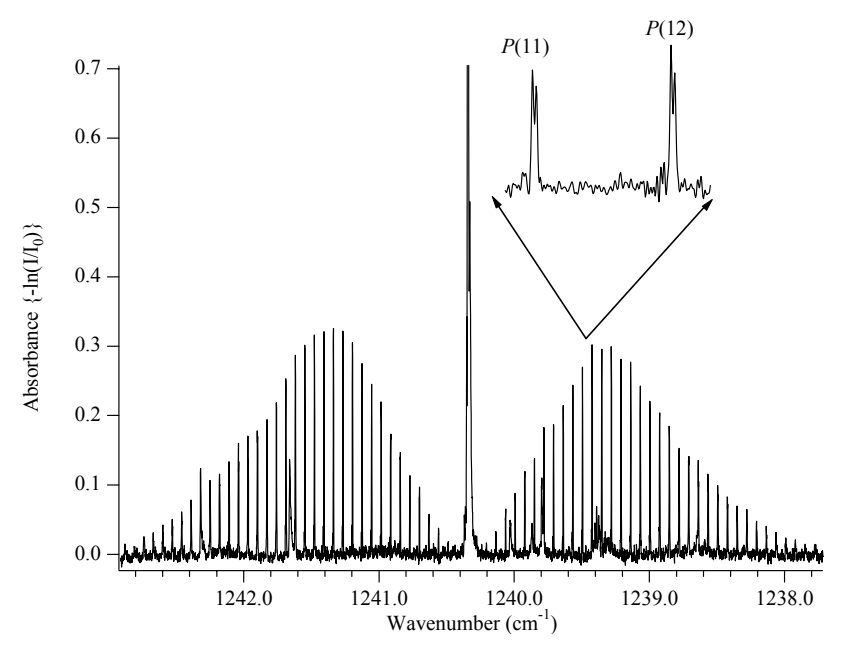

Figure 6.18. Rotationally resolved spectrum of the $v_{13},\left(b_{1 g}\right.$ symmetry under $\left.D_{4 h}\right)$ band of perfluorocyclobutane recorded by crossing jet expansion of 10 percent perfluorocyclobutane mixed in helium with the light from the Fourier transform spectrometer. The rotational temperature is approximately $15 \mathrm{~K}$. The inset figure shows the splitting that can be resolved under these experimental conditions. The rotational transitions in this band obey $\pm \leftarrow$ m selection rules with respect to the ring puckering energy levels. 
can be easily fit to obtain a ground state $B^{\prime \prime}$ value of 0.0354355 (7) $\mathrm{cm}^{-1}$ which is consistent with the $D_{2 d}$, bent ring structure of the molecule. The ring structure of perfluorocyclobutane undergoes a hindered puckering motion and evidence of this can be seen in the splitting of the rotational lines of the $v_{13}$ band (see the inset of Figure 6.18). The rotational transitions in this band $\left(b_{1 g}\right.$ symmetry under $\left.D_{4 h}\right)$ obey $\pm \leftarrow$ m selection rules with respect to the ring puckering energy levels. Analysis of the data is ongoing and ab initio calculations of the molecule's structure, vibrational energy levels, and ring puckering barrier are underway.

Tropolone: A collaboration with Prof. Richard Redington of Texas Tech. University involves the high resolution infrared spectroscopy of tropolone (a seven-member cyclic ring with a ketone and hydroxyl group adjacent to each other). Tropolone exhibits state specific hydrogen tunneling. The effect of small amplitude motions of heavy skeletal atoms on large amplitude hydrogen tunneling motions has been elucidated in Redington and Sams (2000). These tunneling effects are of enormous biological control and signaling relevance and tropolone has provided an excellent intermediary molecule bridging the gap from water to biological molecules. An example of the effects that tunneling has on the IR spectroscopy of Tropolone and may be seen in Figure 6.19.

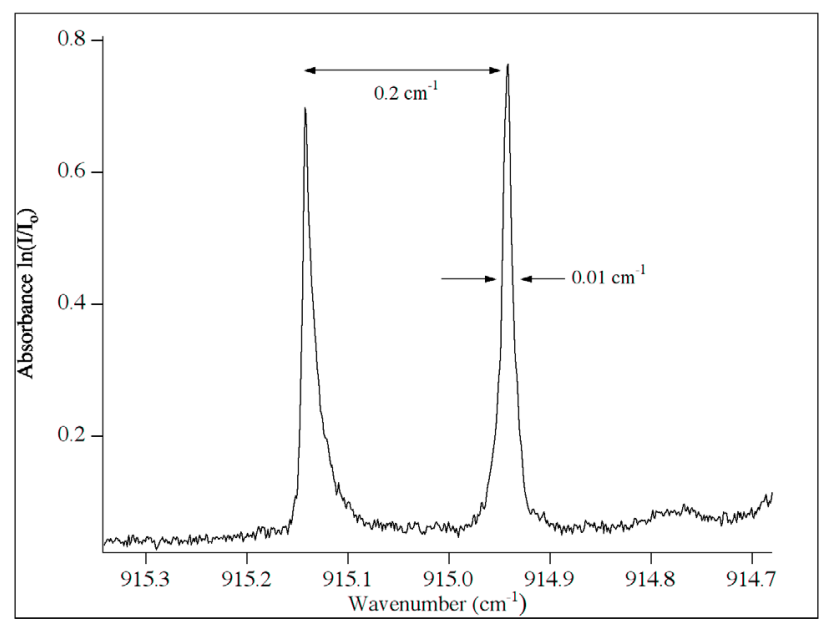

Figure 6.19. Tunneling splitting associated with the $v_{21}$ or $\mathrm{CH}$ wagging mode of tropolone. The tunneling motion involves exchange of a proton between the $\mathrm{OH}$ and $\mathrm{O}$ functional groups appended to the tropolones ring structure.

\section{Applied Research}

Many of the scientific challenges that The Department of Energy must tackle require highly directed, applied research. This may include remediation of legacy sites or the minimization of pollution at operational sites. To this end, the PNNL Infrared Spectroscopy group is involved in a number of DOE sponsored applied research programs that uses infrared optical technologies for characterization and sensing of the environment.

\section{Development of a Quantitative Vapor Phase} Infrared Spectral Library: The National Institute of Standards and Technology (NIST) and the Pacific Northwest National Laboratory (PNNL) are independently creating quantitative, $\sim 0.10 \mathrm{~cm}^{-1}$ resolution, infrared spectral libraries of vapor phase compounds. The NIST library will consist of approximately 100 vapor phase spectra of volatile hazardous air pollutants (HAPs) and suspected greenhouse gases (Chu et al. 1999). The PNNL library will consist of approximately 500 vapor phase spectra associated with DOE's remediation mission (VSBSS 2001).

A critical part of creating and validating any quantitative work involves independent verification based on inter-laboratory comparisons. The two laboratories use significantly different sample preparation and handling techniques. NIST uses gravimetric dilution and a continuous flowing sample while PNNL uses partial pressure dilution and a static sample. Agreement is generally found to be within the statistical uncertainties $(\mathrm{k}=2,2 \sigma)$ of the Beer's law fit and less than 3 percent of the total integrated band areas for the four chemicals used in this comparison. There does appear to be a small (approximately 1.5 percent) systematic difference between the PNNL and NIST data, however. Possible sources of the systematic difference will be discussed as well as technical details concerning the sample preparation and the procedures for overcoming instrumental artifacts.

With the advent of commercially available dispersive infrared spectrometers in the 1950s and Fouriertransform infrared (FTIR) spectrometers in the early 1960s, infrared spectroscopy has become an accepted technique for the identification and quantification of many types of samples. While analysis of liquids and solids is by far the largest application of infrared spectroscopy, vapor phase sample analysis is steadily 
growing. In particular, increased national and international pressure for reduced anthropogenic emissions including hazardous air pollutants, greenhouse gases, and ozone-destroying chemicals has pushed FTIR vapor analysis to new importance. For instance, the Environmental Protection Agency (EPA) has released Method TO-16 for the monitoring of atmospheric gases using long-path, open-path Fourier transform infrared spectroscopy (EPA 1999). Alternatively, EPA Method 320 describes the procedures required for extractive FTIR measurements of vapor phase organic and inorganic emissions (EPA 1993). In addition to FTIR techniques, active open-path monitoring has also been performed using a variety of laser based techniques.

Regardless of the technique used for analysis, the success of both open-path and extractive infrared procedures require a comprehensive and accurate set of reference spectra. These reference spectra must fulfill certain minimum criteria to be generally useful including

- accurate and statistically meaningful absorbance values

- accurate frequency values

- documented sample purity

- sufficient spectral resolution.

There are numerous sources for both hard-copy and digital, vapor-phase, infrared spectral libraries. Some of these libraries are not quantitative, but do use extremely high purity samples, such as The Aldrich Library of FT-IR Spectra of Vapor Phase (Pouchert 1989). Other spectral libraries are quantitative, but are based on a single absorbance spectrum and not on a Beer's law fit to multiple samples with different concentration path length combinations (burdens). Many of these spectral libraries are acquired at relatively low spectral resolution (e.g., $4 \mathrm{~cm}^{-1}$ ) and are inadequate for laser based, tropospheric monitoring techniques.

In an effort to develop a comprehensive set of reference spectra useful for general infrared analysis of pressure broadened, vapor phase samples, the National Institute of Standards and Technology is in the process of creating The NIST Quantitative Infrared Database. In an independent effort, the Pacific Northwest National Laboratory has recently been requested by the Department of Energy to create a quantitative spectral library. Early in this effort, it became apparent that laboratory comparisons between PNNL and NIST would be beneficial. In particular, these comparisons resulted in a better understanding of instrumental artifacts that might otherwise compromise the quantitative nature of the resulting spectral data.

One such molecule that was recently completed for the IR Spectral Library is nitric acid. Nitric acid is extremely reactive and requires careful handling in order to accurately measure its quantitative infrared absorption spectrum. Nitric acid was prepared by dripping concentrated sulfuric acid onto sodium nitrate. The resulting $\mathrm{HNO}_{3}$ vapor was then condensed and kept frozen until needed. The pressure broadened (1 atm. UHP nitrogen) spectra were recorded for a variety of partial pressures of $\mathrm{HNO}_{3}$ ranging from 0.1 to 5 torr. These individual spectra were then fit to Beer's law plot using a weighted linear least squares algorithm. The resulting slope at each frequency channel $\left(6,500-600 \mathrm{~cm}^{-1}\right)$ is the statistical value for the absorption coefficient. One such fitted spectrum for $\mathrm{HNO}_{3}$ at $25^{\circ} \mathrm{C}$ appears in Figure 6.20. Typical uncertainties for the integrated band areas are found to be on the order of 3 percent $(2 \sigma)$.

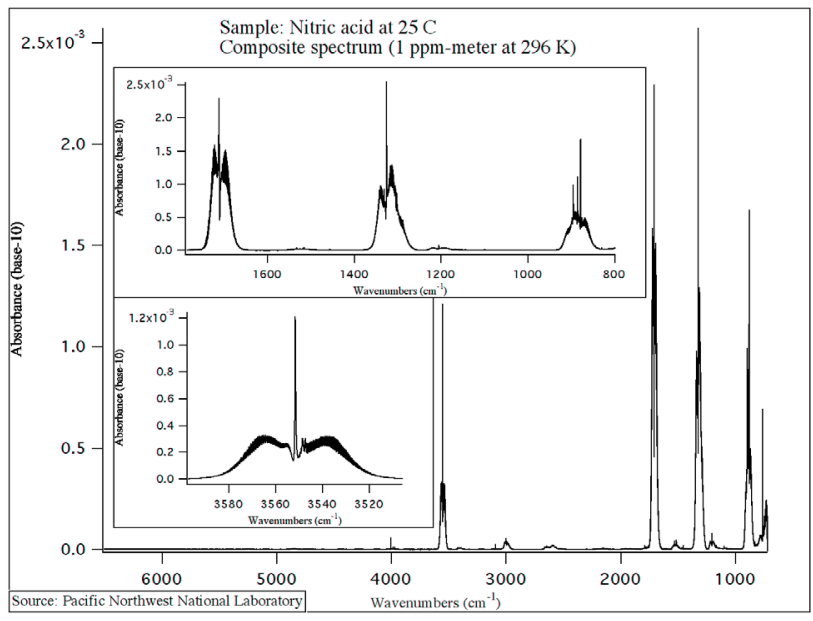

Figure 6.20. Representative spectrum from the PNNL/DOE Infrared Spectral Library. This spectrum corresponds to a statistical fit to Beer's law for many individual absorbance spectra at different partial pressures of nitric acid. 


\section{Identifying and Fixing Intensity Artifacts in Gas Phase FTIR Spectroscopy: FTIR spectroscopy} retains many advantages but is still susceptible to spectral artifacts such as "aliasing" when light of a given frequency shows up at the wrong wavelength (e.g., due to an insufficient digitization rate) as well as the common problem of non-linear light response from some detectors, most notably photoconductive mercury cadmium telluride (MCT) detectors. Although both the aliasing and non-linearity artifacts are understood and modern software/hardware exists to avoid these pitfalls, it is still important for the user to be aware of them and to understand their origin. In the present research, however, we report observation of two additional artifacts, both associated with the single limiting aperture in most commercial FTIRs. The first of these has been observed and noted in the literature (Griffiths and de Haseth 1986; Birk et al. 1996), but we present a more detailed description as to how the annulus of the aperture can be considered as a $\sim 300 \mathrm{~K}$ black body source that contributes to (and consequently distorts) the spectrum. The second effect also originates from the aperture, namely when light is reflected from its surface that light can betwice modulated by the interferometer (Giver et al. 1992; Birch and Clarke 1995; Learner and Brault 1996). The two effects are considered in turn, as well as their solutions.

At even moderately high resolution the image seen by a liquid-nitrogen cooled infrared detector such as an MCT actually consists of an inner hot $(\sim 1500 \mathrm{~K})$ circle and an outer warm $(\sim 300 \mathrm{~K})$ annulus. The inner circle is the aperture into which the IR source is focused from the source, typically having a temperature near $1500 \mathrm{~K}$. About this "hot" inner circle is an annulus whose outer imaged dimension is determined by the detector element size and the spectrometer demagnification. For many commercial spectrometers capable of moderately high resolution (e.g., 0.125 to $0.10 \mathrm{~cm}^{-1}$ ), an $\sim \mathrm{f} / 4$ optical speed is typical. For a typical demagnification of say, 4 , and a typical MCT element size of $1 \times 1 \mathrm{~mm}$, the image the cooled detector sees would be a $4 \times 4 \mathrm{~mm}$ square at approximately $300 \mathrm{~K}$ due to the cold-shield, as well as an inner image near $1500 \mathrm{~K}$ due to the focused source. Typical aperture settings for gas phase work at $1 / 8$ or $1 / 10 \mathrm{~cm}^{-}$ ${ }^{1}$ resolution would prescribe an aperture on the order of 1 to $1.5 \mathrm{~mm}$ diameter. The "warm aperture" problem manifests itself in the spectra in rather subtle ways, since the spectrometer thus effectively has two sources at two different temper-atures. It is recalled that in order to achieve a certain spectral resolution in a Michelson interferometer, the aperture diameter $A$ cannot exceed a certain value (details of the derivation can be found, for example, Kaldor and Maki [1973]): $A=2 * F\left(\Delta \sigma / \sigma_{\max }\right)^{1 / 2}$

Here $F$ is the spectrometer focal length, $\Delta \sigma$ is the desired resolution in $\mathrm{cm}^{-1}$, and $\sigma_{\max }$ is the maximal frequency to be measured (in $\mathrm{cm}^{-1}$ ). For a given spectrometer and with measurement at a given resolution, this equation dictates the largest allowed aperture that will still allow one to fulfill the resolution condition up to the specified wave number. Should the (effective) aperture be made larger than this, the off-axis rays entering the spectrometer cause two distortions: First, the instrumental line width will be broader, especially at the short wavelength end of the spectrum, and second, the line shape can be distorted. Albeit a weaker thermal source, the outer annulus is effectively an unapertured source distorting the line shape and intensity due to off-axis rays. The effect of the thermal contamination on spectral line shape is often a tail to longer wavelengths in the region of thermal output to the detector cutoff, e.g., 1200 to $600 \mathrm{~cm}^{-1}$.

For the quantitative gas phase applications in our laboratories, we have successfully implemented a solution first suggested by others (Birk et al. 1996; Giver et al. 1992) to remove this artifact. By placing a second aperture after the interferometer, the "warm aperture" problem is minimized. So long as the étundue of the second focusing mirror/aperture system is chosen to match that of the first, the detector sees only the source light focused through the first aperture, and not the annulus. Any thermal radiation from the second aperture is not modulated by the interferometer and is thus discarded as a DC signal during Fourier transformation. With careful alignment, we observed light losses of $<25$ percent, even though four additional mirrors and an aperture were installed. Before the second aperture was installed, Figure 6.21 shows the single beam spectra recorded with the infrared source "on" and "off," showing significant contribution to the spectrum in the 600 to $1200 \mathrm{~cm}^{-1}$ region. Source off spectra were produced by switching the source mirror from the glowbar position to an extinguished tungsten lamp. With the second aperture in place, the effect is reduced by more than an order of magnitude. 


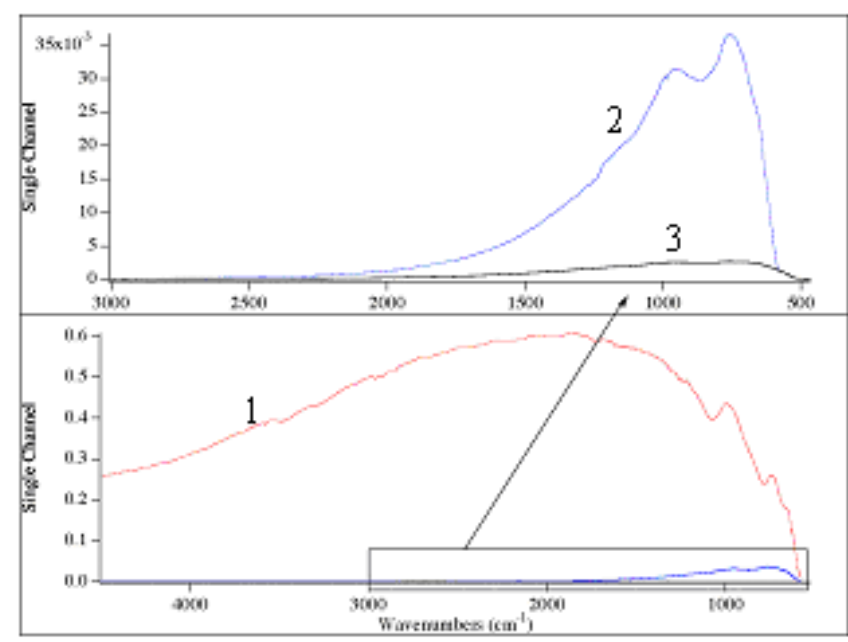

Figure 6.21. Single beam spectra showing the effect of thermal contribution from the aperture. Lower and upper traces: traces include source on without $2^{\text {nd }}$ aperture, source off without $2^{\text {nd }}$ aperture, source off with $2^{\text {nd }}$ aperture.

We can further observe the effect of this thermal contamination by recording spectra of ethene with the infrared source both on and off. With the second aperture mechanism in place, the intensities and line shapes for the $\mathrm{C}_{2} \mathrm{H}_{4}$ absorption lines do not change significantly for those at shorter wavelengths (e.g., shorter than $5 \mu \mathrm{m}$ ). As seen in Figure 6.22, for the fingerprint region, there is a serious discrepancy due to the black body distribution of radiation emitted from the back side of the warm aperture. These discrepancies in intensity can be 10 percent or more for features appearing below $1000 \mathrm{~cm}^{-1}$.

Only half of the modulated light that exits a Michelson interferometer is directed to the detectorthe other half is directed back towards the aperture and source. This is well known, but not considered since the light is normally not used. However, we observed a subtle yet reproducible error in the spectra that can clearly be attributed to infrared light being twice modulated. The effect seen on the Bruker IFS $66 \mathrm{v}$ is presumably seen in all single aperture instruments. Figure 6.23 shows for both the $\mathrm{CO}$ and $\mathrm{CO}_{2}$ lines an "alias" at exactly twice the fundamental frequency. We emphasize that these are not overtone lines (at less than $2 \mathrm{f}$ due to anharmonicity) but arise from light twice modulated by the interferometer. We also point out that although the effect is only seen at the few percent level for strongly absorbing lines, it in fact indicates that the entire spectrum suffers from

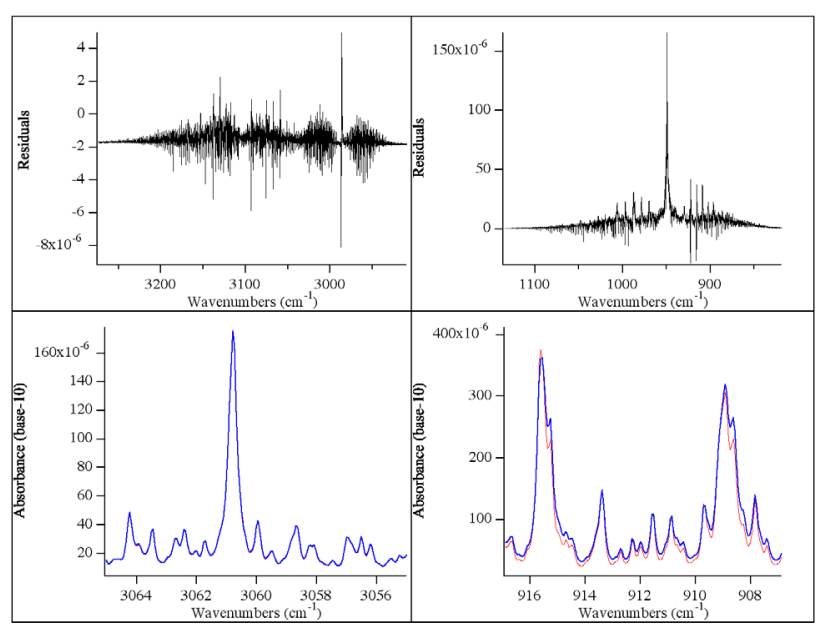

Figure 6.22. Absorbance spectra of $\mathrm{C}_{2} \mathrm{H}_{4}$ in the MWIR and LWIR (fingerprint) region showing the effect of the warm aperture. Lower two traces correspond to ethene spectra before and after installation of $2^{\text {nd }}$ aperture. Upper two traces correspond to residuals of the before- $2^{\text {nd }}$-apertrure minus the after- $2^{\text {nd }}$-aperture. Note the significant increase in residuals at the lower frequencies.

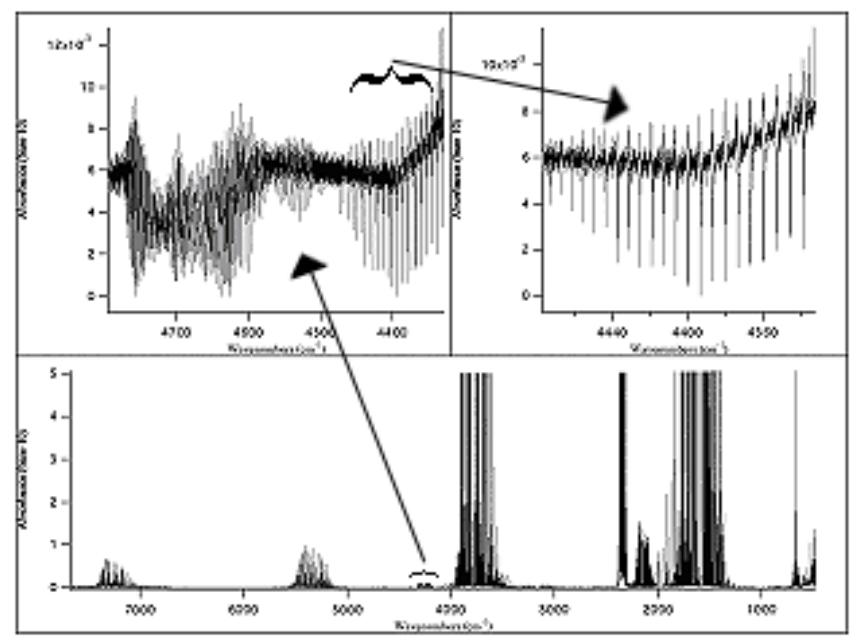

Figure 6.23. The spectral effect of double modulation or "aperture ghosting" due to reflection off the back side of the aperture. The light passes the interferometer twice and shows up at twice its natural frequency.

intensity contributions due to light at half the frequency being twice modulated. This in turn implies that the recorded intensity (or absorbance values) suffer from an additional systematic error on the order of a few percent. One solution to this would be to tilt the aperture relative to beam normal, but this in turn distorts the image. We found the best method was to 
either black anodize the rear side of the aper-ture or (as was used for the second Jacquinot stop) to form a conical, reflective piece on the side facing the interferometer to reflect rays at oblique angles.

\section{Rapid Standoff Detection of Chemical Spills:}

Rapid standoff detection of soil surface contaminants would be useful, for example, for monitoring the accretion of low volatility compounds on the ground near factories, determining the content and extent of chemical spills, verifying treaty compliance at suspected weapons of mass destruction production sites, and application-monitoring of agri-chemicals such as fertilizers, pesticides, and herbicides. We say "would" because there has been comparatively little progress made in standoff detection in these situations. Traditionally, soil samples have been collected and brought back to the laboratory for analysis, which is reasonable since qualitative and quantitative techniques, such as gas chromatography and mass spectrometry and their hybrids, are very sensitive and selective. But where to collect the samples? Treaty verification, for example, allows for only a limited time on-site to collect samples. If the ground could be scanned using infrared reflection spectroscopy to look for classes of compounds such as phosphates, phosphonates, nitrates, etc. - or better yet, specific compounds - that appear with concentrations well above the anticipated background, sample collection could be hastened considerably. Using Laboratory Directed Research and Development (LDRD) funding, our work on this subject thus far has involved laboratory proof-of-principle experiments to examine the feasibility of using infrared reflection spectroscopy as a technique for detecting chemical compounds on the surface of soils. We present reflection spectral signatures for several compounds, determine detection limits, and discuss the effects of angle of incidence and polarization on detectability (Blake and Gassman 2002).

A bench top Fourier transform infrared spectrometer and specular reflection accessory has been used to record reflection spectra of several chemical compounds coating a loamy, psamment soil (we refer to this as Hanford soil). Unpolarized reflection spectra were recorded between 4600 and $500 \mathrm{~cm}^{-1}$ with a resolution of $4 \mathrm{~cm}^{-1}$ for the compounds dimethyl methylphosphonate, trimethyl phosphate, methylphosphonic acid, 2,2'-thiodiethanol, diazinon, diesel fuel, and ammonium nitrate mixed separately into soil samples with concentrations of 10,5 , and $1 \mathrm{mg}$ of analyte compound per gram of soil. The soil reflection spectra are compared to liquid or solid transmission spectra of the pure compound and frequency shifts and relative intensity changes for the absorption features are noted. As an example of detection sensitivity, we have estimated that we can detect 300 nanograms of dimethyl methylphosphonate on the surface layer of soil in the focal spot $\left(15 \mathrm{~mm}^{2}\right)$ of the reflection accessory using one of DMMP's $\mathrm{C}-\mathrm{H}$ stretch modes (see Figure 6.24). The signal-tonoise (peak-to-peak) of this spectral feature under
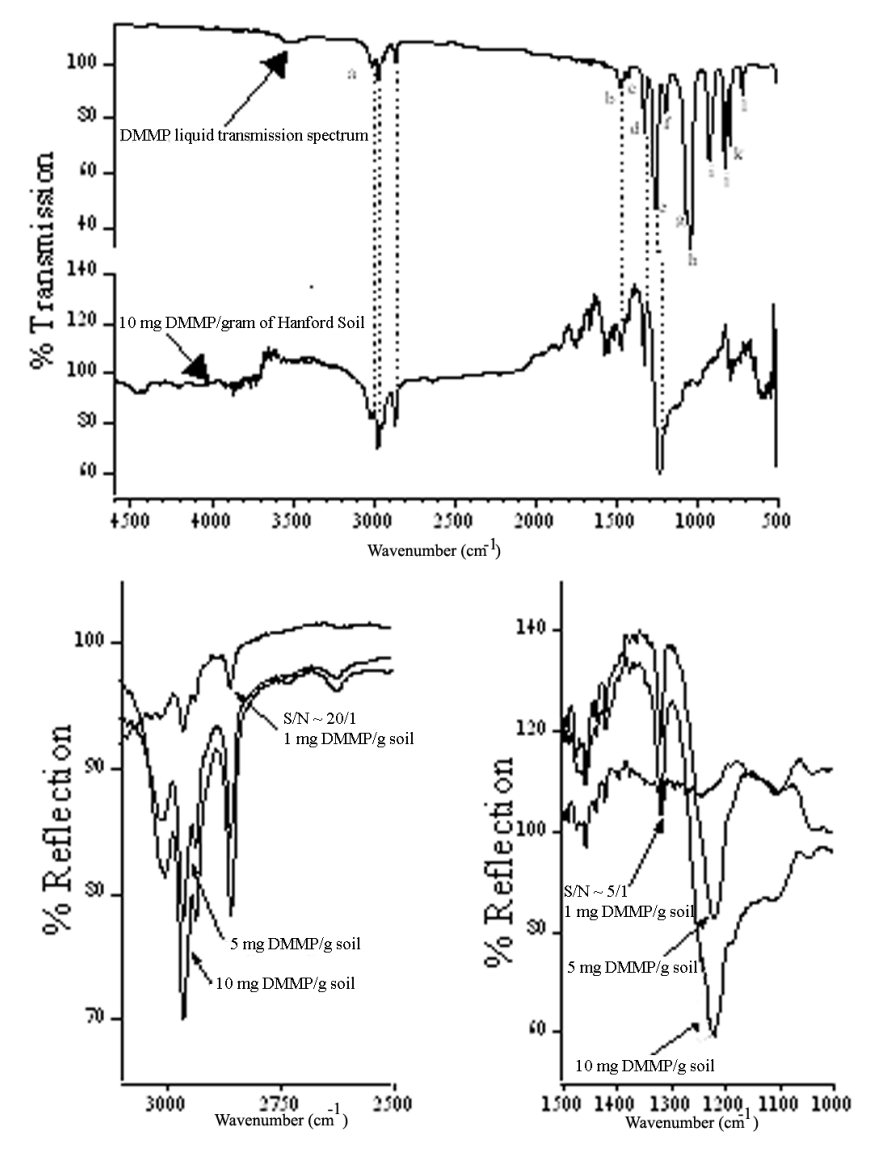

Figure 6.24. Improvement in signal-to-noise due to optimal filtering is illustrated by comparing spectra collected using: (a) background subtraction only; and (b) two spectra each undergoing background subtraction followed by application of optimal filtering $(\mathrm{N}=1000$ scans each). The dominant spectroscopic feature is due to ambient $\mathrm{CH}_{4}$ absorption at $1245.220 \mathrm{~cm}^{-1}$ (HITRAN), whereas the weak absorption feature at $1.45 \mathrm{~ms}$ is from ambient $\mathrm{CO}_{2}$ at $1245.415 \mathrm{~cm}^{-1}$. 
these circumstances would be $3 / 1$. We also estimate that the flux of infrared photons reaching the soil is $10^{14}$ photons $/ \mathrm{sec} / \mathrm{cm}^{2}$. We have also recorded polarization-resolved reflection spectra of the uncoated soil and coarse and fine grained quartz sands as a function of angle of incidence and reflection and have determined the degree of polarization for light reflected off of these materials at frequencies associated with volume scattering and surface scattering features. As might be expected, the volume scattering features show a significant depolarization of light: the degree of polarization after reflection is $<20$ percent and the surface scattering features retain a much higher degree of polarization upon reflection, $>75$ percent. We have also recorded polarization resolved spectra of tributyl phosphate on the soil and found significant differences between the $s$ - and $p$-polarized spectra. This fact could be used to employ polarization modulation detection to improve detection sensitivity.

The Hanford soil sample was obtained from the arid shrub-steppe environment of southeastern Washington State, USA. The soil is a mixed, thermic, loamy psamment derived from 0 to $15 \mathrm{~cm}$ depth. The soil used for spectroscopic measurements was sieved to be less than $500 \mu \mathrm{m}$ equivalent spherical diameter (ESD).

\section{A Laser Based, Hollow Waveguide System for} Environmental Monitoring: Compact and portable laser sensing devices are important for in situ environmental sensing, industrial hygiene monitoring, medical applications, intelligence gathering operations, and laboratory analysis. Mid-infrared laser diodes are ideal light sources for this kind of detection because of their high spectral brightness and the molecular specificity available in this frequency range. Even so, many detection scenarios require detecting compounds in the part per million concentration range or smaller. This in turn requires pathlengths of many meters to achieve the required detectivity. Typically, multireflection cells such as White or Herriott cells are used to increase pathlength, but these are heavy, bulky, expensive, and require careful alignment. In the past decade, high efficiency hollow waveguide fibers that transmit light in the midinfrared have become commercially available. By flowing gas containing the analyte compound through the fiber and launching laser light into the fiber through windows on the ends of the fiber, a compact, lightweight, and relatively inexpensive long path-length absorption cell can be used. The waveguide fibers have internal bore sizes on the order of $1 \mathrm{~mm}$, can be cut to any length, and through whispering gallery mode reflections in the waveguide, absorption enhancement can be achieved. Figure 6.25 shows a schematic diagram of the infrared laser diode and hollow waveguide system that we have constructed.

Using this system spectroscopy can be performed either as direct absorption or as wavelength modulated absorption. Figure 6.26 shows a spectrum of the ${ }^{2} \Pi_{3 / 2}$, $\mathrm{v}=1-0, R(8.5)$ transition of NO at $1906.73 \mathrm{~cm}^{-1}$. The spectrum was recorded by flowing a $1 \mathrm{ppm}$ NOin-nitrogen mixture through the hollow waveguide fiber and launching light from a tunable lead-salt laser through the fiber. Experiments are continuing to characterize this system for performing in situ chemical sensing.

\section{Quantum Cascade Laser Based System for} Atmospheric Monitoring: Optical feedback to the laser source in tunable diode laser spectroscopy (TDLS) is known to create intensity modulation noise due to étaloning and optical feedback (i.e., multiplicative technical noise) that usually limits spectral signalto-noise $(\mathrm{S} / \mathrm{N})$. The large technical noise often limits absorption spectroscopy to noise floors 100 -fold greater than the Poisson shot noise limit due to fluctuations in the laser intensity. The high output powers generated from quantum cascade (QC) lasers, along with their high gain, makes these injection laser systems especially susceptible to technical noise.

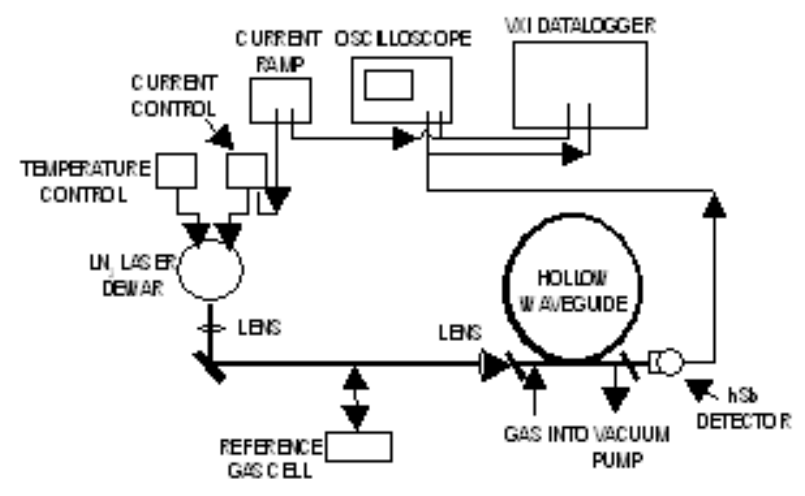

Figure 6.25. Schematic diagram of the infrared diode laser and waveguide set up. The laser is a lead-salt diode laser operated at liquid nitrogen temperature. The waveguide is three meters in length and has a bore of $1 \mathrm{~mm}$ and the detector is a liquid nitrogencooled $\mathrm{HgCdTe}$ detector. 


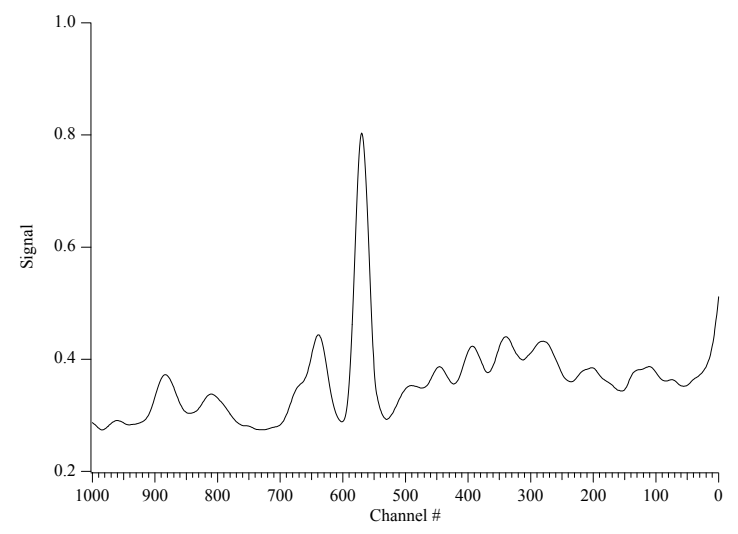

Figure 6.26. Spectrum of the ${ }^{2} \Pi_{3 / 2}, v=1-0, R(8.5)$ transition of $\mathrm{NO}$ at $1906.73 \mathrm{~cm}^{-1}$. The spectrum was recorded by flowing a $1 \mathrm{ppm}$ NO in nitrogen mixture through a hollow waveguide fiber and launching light from a tunable lead-salt laser throught the fiber. The laser was frequency modulated at $300 \mathrm{kHz}$ and the infrared detector signal demodulated by a HewlettPackard 8563A spectrum analyzer. The laser frequency was ramped over the transtion at a rate of $1.131 \mathrm{kHz}$ and 10,000 scans were coadded in 3.6 seconds. Wavenumbers are increasing left to right on the bottom axis.

We have developed a method of using optimal filtering to reduce technical noise (Anderson and Moore 1979). Observed $\mathrm{S} / \mathrm{N}$ enhancements ranging from $\sim 20$ percent to $\sim 50$ percent have been noted. The degree to which optimal filtering enhances $\mathrm{S} / \mathrm{N}$ depends on the similarity between the Fourier components of the technical noise and those of the signal, with lower $\mathrm{S} / \mathrm{N}$ enhancements observed for more similar Fourier decompositions of the signal and technical noise. In our work we have demonstrated that optimal filtered spectra for both time and intensity is linear. This was accomplished by creating a synthetic spectrum for the species being studied $\left(\mathrm{CH}_{4}, \mathrm{~N}_{2} \mathrm{O}\right.$, $\mathrm{CO}_{2}, \mathrm{H}_{2} \mathrm{O}$ in ambient air) using line-positions and line-widths with an assumed Voigt-profile from a commercial database (HITRAN). Agreement better than 0.036 percent in wavenumber, and 1.64 percent in intensity (up to a 260 -fold intensity ratio employed), was observed. Our results suggest that rapid ex post facto digital optimal filtering can be used to enhance $\mathrm{S} / \mathrm{N}$ for routine trace gas detection. An example of spectral $\mathrm{S} / \mathrm{N}$ enhancement is shown in Figure 6.27 for a (a) non-optimal filtered, and (b) optimal filtered, spectrum.
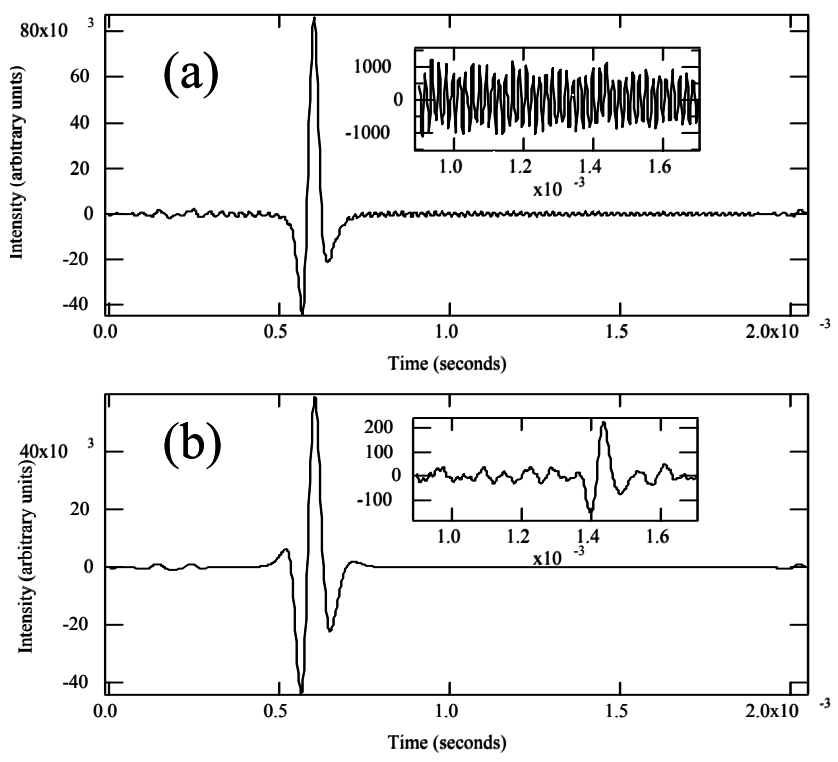

Figure 6.27. Improvement in signal-to-noise due to optimal filtering is illustrated by comparing spectra collected using: (a) background subtraction only and (b) two spectra each undergoing background subtraction followed by application of optimal filtering ( $N=1000$ scans each). The dominant spectroscopic feature is due to ambient $\mathrm{CH}_{4}$ absorption at $1245.220 \mathrm{~cm}^{-1}$ [HITRAN], whereas the weak absorption feature at $1.45 \mathrm{~ms}$ is from ambient $\mathrm{CO}_{2}$ at $1245.415 \mathrm{~cm}^{-1}$.

\section{References}

Anderson, B. D. O, and J. B. Moore, Optimal Filtering, Prentice-Hall Inc., Englewood Cliffs, NJ (1979).

Barber, J., E. t. H. Chrysostom, A. Masiello, J. W. Nibler, A. G. Maki, A. Weber, T. A. Blake, R. L. Sams; manuscript in preparation.

Birch, J. R., and F.J.J. Clarke, Spectroscopy Europa 7, 16-22 (1995).

Birk, M., D. Hausamann, G. Wagner and J.W. Johns, Appl. Opt. 35, 2971, (1996).

Blake, T. A. and P. L. Gassman, SPIE Proceedings 4577, 239 (2002).

Chrysostom, E. H., N. Vulpanovici, A. Masiello, J. Barber, J. W. Nibler, A. Weber, A. G. Maki, T. A. Blake, J. Mol Spectrosc. 210, 233 (2001). 
Chu, P. M., F. R. Guenther, G. C. Rhoderick and W. J. Lafferty, J. of Res. of the NIST, 104, 59-81 (1999).

Finlayson-Pitts, J., J. N. Pitts, Chemistry of the Upper and Lower Atmosphere, Academic Press, San Francisco (2000).

Fisher, G., R. L. Purchase, D. M. Smith, J. Mol. Struc. 405, 159 (1997).

Giver, L. P., F.P.J. Valero, D. Goorvitch, F. S. Bonomo, J. Optical Soc. Amer. B 1, 715 (1984).

Giver, L. P., C. Chackerian, Jr. and M. N. Spencer, "Thermal Contamination of BOMEM FTIR Line Shapes," High Resolution Fourier Transform Spectroscopy 1992 Technical Digest Volume 21, Optical Society of America (1992).

Goldman, C. P. Rinsland, A. Perrin, J-M. Flaud, J. Quant. Spectrosc. Radiat. Transfer 60, 851 (1998).

Griffiths, P. R., and J. A. de Haseth, "Fourier Transform Infrared Spectroscopy," (J. Wiley and Sons Inc. New York (1986).Kaldor, A. G. Maki, J. Mol. Struc. 15, 123 (1973).

Learner, R. C. and J. W. Brault, Appl. Opt. 35, 2947-2954 (1996).
Maki, G., T. A. Blake, R. L. Sams, N. Vulpanovici, J. Barber, E. t. H. Chrysostom, A. Masiello, J. W. Nibler, A. Weber, J. Mol Spectrosc. 210, 240 (2001).

Ortigoso, J., R. Escribano, A. G. Maki, J. Mol. Spec. 138, 602 (1989).

Pouchert, C. J., The Aldrich Library of FT-IR Spectra Vapor Phase, Edition 1, Vol. 3, The Aldrich Chemical Comp. Inc.: Milwaukee, WI (1989).

Redinton, R. L. and R. L. Sams, J. Phys. Chem B 106, 7497-7511 (2002).

U.S. Environmental Protection Agency (EPA) Method 320, Compendium of Methods for the Determination of Toxic Organic Compounds in Ambient Air, Second Edition, (EPA/625/R-96/010b) (1999).

U.S. Environmental Protection Agency (EPA) Method 320, Measurement of Vapor Phase Organic and Inorganic Emissions by Extractive Fourier Transform Infrared Spectroscopy (1993) http://www.epa.gov. 


\section{Appendix}




\section{Chemical Structure and Dynamics Staff}

\section{Associate Director, EMSL}

Steven D. Colson

(509) 376-4598

steven.colson@pnl.gov

B.S. Utah State University 1963; Ph.D.

California Institute of Technology 1968; Post-

doctoral Fellow, National Research Council

(Ottawa) 1968; Assistant Professor to

Professor of Chemistry, Yale University, 1968-1989; joined PNNL as Associate

Director for Chemical Structure and Dynamics June 1989. Research interests: photochemistry, photophysics, and molecular dynamics; electronic structures of molecules; processes at the molecule/surface interface.

\section{Program Manager}

Roy E. Gephart

(509) 376-1421

roy.gephart@.pnl.gov

B.A. (Geology) Miami University 1971; M.S.

(Geohydrology) Wright State University 1974; 1974-1987 geohydrologist and line manager with prime Hanford contractors; joined PNNL in 1987 as Program Manager for Environmental Restoration studies. Numerous local to international science outreach and writing awards. Interests: groundwater hydrology, geology, science-policy linkages, science education, nuclear waste issues.

\section{Operations Administrator}

Cynthia A. Irwin

\section{Office Support}

Jo Breneman (to July 2001)

Jayme R. Crow

Brenda K. Plata (from August 2001)

Kathelene J. Valdez (from August 2001)

Anna Akopova (COE to June 2001)

\section{Staff}

Stephan E. Barlow

(509) 376-9051

se.barlow@pnl.gov

B.A. Southern Oregon State College 1976;

Ph.D. University of Colorado 1984, Post-

doctoral Fellow 1984-1989; Senior Instructor

in Chemistry, University of Denver 1989

1991; joined PNNL June 1991. Research

interests: development and application of

mass spectrometric techniques to gas-phase

chemical kinetics, reaction mechanisms, and

structure.

James P. Cowin

(509) 376-6330

ip.cowin@pnl.gov

B.S. Case Western Reserve University 1974;

Ph.D. University of Chicago 1981; Post-

doctoral Fellow, University of Toronto 1981-

1983; Assistant Professor of Chemistry,

University of California, Santa Barbara, 19831990; joined PNNL July 1990. Research interests: molecular motions at interfaces, especially energy and charge transfer, bond formation dynamics, and light-stimulated surface reactions.

Robert S. Disselkamp

(509) 372-6061

robert.disselkamp@.pnl.gov

B.S. University of Minnesota 1985; Ph.D. Indiana University 1990; Postdoctoral Fellow, Colorado State University 1990-1993 and University of Colorado 1993-1995; Assistant Professor, University of Alaska, 1995-1998; joined PNNL 1998 on a joint appointment with Atmospheric Sciences group and CS\&D. Research interests: atmospheric heterogeneous chemistry; atmospheric aerosols and clouds; optical properties of particle ensembles; remote sensing of clouds. 
Wayne P. Hess

(509) 376-9907

wayne.hess@pnl.gov

B.A. University of Colorado 1981; M.S.

University of Oregon 1983; Ph.D. University

of Colorado 1988; Postdoctoral Fellow,

Sandia Livermore Combustion Research

Facility 1988-1990; joined PNNL January

1990. Research interests: modeling con-

densed-phase chemistry through the study of energetic reactive processes in molecular solids and thin films (dissociation and fragmentation, electronic and vibrational energy transfer), using laser-induced desorption and ablation of adsorbed molecules and solid surfaces.

Gary R. Holtom

(509) 376-5331

gary.holtom@pnl.gov

B.A. Pfeiffer College 1969; Ph.D. University of California, Berkeley, 1978; Postdoctoral Fellow, Wesleyan University 1978-1979; Head of Laser Operations, University of Pennsylvania Regional Laser and Biotechnology Laboratory, 1979-1989; joined PNNL September 1989. Research interests: shortpulse laser spectroscopy; condensed-phase photochemistry, in particular the dynamics of electronically excited organic molecules.

Stephen A. Joyce

B.S. Boston College 1982; Ph.D. M.I.T. 1987; Postdoctoral Fellow, National Institute of Standards and Technology 1987-1989; Sandia National Laboratories 1989-1991; PNNL Oct. 1991 - April 2001. Research interests: scanning probe microscopies for studying molecularly-resolved chemistry on solid surfaces. Now at Los Alamos National Laboratory.
Bruce D. Kay

(509) 376-0028

bruce.kay@pnl.gov

B.S. University of Illinois 1976; Ph.D.

University of Colorado 1982; Senior Member

of the Technical Staff, Sandia National

Laboratories, 1982-1991; joined PNNL

November 1991. Research interests: experimental studies employing molecular beamsurface scattering and laser spectroscopic techniques aimed at elucidating the chemical kinetics and dynamics of adsorption, desorption, diffusion, phase transformation, and solvation at model aqueous and oxide interfaces relevant to the environment; nanoporous thin-film synthesis and characterization; hydrogen in and on metals; phase transitions in amorphous materials and deeply supercooled liquids.

Gregory A. Kimmel

(509) 376-2501

gregory.kimmel@pnl.gov

B.S. Cornell University 1984, Ph.D. 1992;

Postdoctoral Fellow PNNL, 1992-1995; joined PNNL staff as Senior Research Scientist September 1995. Research interests: gas-surface dynamics; energetic processes at surfaces and interfaces; nanoscience.

Hong (Peter) Lu

(509) 376-3897

hong.lu@pnl.gov

B.A. Peking University 1982, M.Sc. 1984; M. Phil. Columbia 1987, Ph.D. 1991; Postdoctoral Fellow, Northwestern 1991-1995; Postdoctoral Fellow, PNNL, 1995-1996; joined PNNL staff as Senior Research Scientist November 1996. Research interests: near-field and confocal single-molecule linear and nonlinear spectroscopy and imaging; single-molecule enzymatic and protein conformational change dynamics; biomolecular interaction and recognition dynamics; singlemolecule interfacial electron transfer dynamics. 
Steven W. Sharpe

(509) 376-7769

sw.sharpe@pnl.gov

B.S. University of Bridgeport 1979; Ph.D.

State University of New York at Stony Brook

1986; Postdoctoral Fellow, University of

Southern California 1987-1990; joined PNNL

May 1990. Research interests: high-

resolution molecular spectroscopy; remote

sensing.

Lai-Sheng Wang

(509) 376-8709

ls.wang@pnl.gov

B.S. Wuhan University 1982; Ph.D.

University of California, Berkeley, 1989;

Postdoctoral Fellow, Rice University 19901992; joint Washington State University/

PNNL appointment January 1993. Research interests: nanoclusters and nanomaterials; cluster models of environmentally important materials; electronic structure and chemical bonding of atomic clusters; tetracoordinate planar carbon molecules; aromaticity in metallic clusters; photoelectron spectroscopy and pulsed laser vaporization techniques; multiply charged anions; repulsive Coulomb barriers; ion solvation and H-bonding; gasphase studies of solution-phase chemistry, electrospray, and ion trap techniques.

\section{Research Scientists}

Kenneth M. Beck

(509) 376-9152

kenneth.beck@pnl.gov

B.A. Northwestern University 1972; M.S.

University of Illinois at Chicago 1985, Ph.D.

1988; Postdoctoral Fellow University of

Pennsylvania 1988-1990, PNNL 1990-1991;

Asst. Professor, University of Central Florida

1991-1995; PNNL 1996; joined PNNL

September 1996.
Thomas A. Blake

(509) 376-8974

ta.blake@pnl.gov

B.S. University of Bridgeport 1981; Ph.D.

Wesleyan University 1989; Postdoctoral

Fellow, University of Washington 1988-1991;

NRC Research Associate, NASA Ames

Research Center 1991-1994; joined PNNL

September 1994.

John L. Daschbach

(509) 376-2467

john.daschbach@pnl.gov

B.S. Lewis \& Clark College, 1983, Ph.D.

University of Utah, 1989; Postdoctoral Fellow,

University of Utah, 1990-1991; Postdoctoral

Fellow, University of Oregon, 1991-1993;

joined PNNL January 1994; joined PNNL

December 2000.

Zdenek Dohnálek

(509) 376-3726

zdenek@pnl.gov

B.S. Institute of Chemical Engineering,

Prague, 1989, M.S. 1991; Ph.D. University of

Pittsburgh 1997; Postdoctoral Fellow, CS\&D, with Bruce Kay, 1997-2000; joined PNNL

July 2000.

Martin J. Iedema

(509) 376-6039

martin.iedema@pnl.gov

B.S. University of Southern California 1988;

M.S. Washington State University, 1999;

Technician, Mt. Wilson Observatory $1987-$

1990; joined PNNL September 1990.

Alan G. Joly

(509) 376-7707

agjoly@pnl.gov

B.S. University of Rochester 1985; Ph.D.

M.I.T. 1990; Postdoctoral Fellow, PNNL

1990-1993; joined PNNL June 1993.

Alexander Laskin

(509) 376-8741

alexander.laskin@pnl.gov

M.S. Technical University of Leningrad 1991;

Ph.D. Hebrew University, Jerusalem, 1998;

Postdoctoral Fellow, CS\&D, with Jim Cowin, 1999-2001; joined PNNL September 2001. 
Galya Orr

(509) 376-9592

galya.orr@pnl.gov

B.S. The Technion, Israel Institute of Technology 1984; Ph.D. University of Arizona 2002; Graduate Student, PNNL 1999-2000; joined PNNL January 2001.

Nikolay G. Petrik

(509) 376-5379

nikolai.petrik@pnl.gov

M.S. St. Petersburg Institute of Technology 1977, Ph.D. 1980, D.Sc. 1994; Senior

Scientist with All-Russian Project and Research Institute of Complex Power Technology (VNIPIET), St. Petersburg, 19821998; SABIT (Special American Business Internship Training Program) internee at PNNL with Thom Orlando, 1996; Visiting Scientist 1997-2000; joined PNNL February 2000 .

Marianne Sowa Resat

(509) 376-9104

Marianne.sowa-resat@pnl.gov

B.S. Spring Hill College (Alabama) 1989;

Ph.D. State University of New York at Stony

Brook 1994; Postdoctoral Fellow, University of California at San Diego 1994-1996; Assistant Professor, Bosphorus University, Istanbul, 1997-1999; Postdoctoral Fellow PNNL 1999-2000; joined PNNL October 2000.

Robert L. Sams

(509) 376-8995

robert.sams@pnl.gov

B.A. Western Washington University 1962;

M.S. University of Washington 1967; National

Bureau of Standards/National Institute of

Standards and Technology 1967-1995; AWU

Visiting Scientist Fellowship, PNNL, 1995-

1998; joined PNNL October 1998.

R. Scott Smith

(509) 376-0907

scott.smith@pnl.gov

B.S. Northern Arizona University 1983; Ph.D.

University of Utah 1988; Postdoctoral Fellow, University of Arizona 1988-1989, PNNL 1990-1992; joined PNNL October 1992.
Russell G. Tonkyn

(509) 376-8817

rg.tonkyn@pnl.gov

B.A. Reed College 1978; Ph.D. University of Wisconsin 1988; Postdoctoral Fellow, Brookhaven National Laboratory 1988-1991, PNNL 1991-1992; joined PNNL November 1992.

XueBin Wang (509) 376-8958

xuebin.wang@pnl.gov

B.S. University of Science and Technology, Hefei, 1988; Ph.D. Institute of Chemistry, Chinese Academy of Sciences 1995; Postdoctoral Fellow, Columbia University 19951997; Postdoctoral Fellow, PNNL, with LaiSheng Wang, 1997-2000; Research Scientist, Washington State University, from June 2000.

\section{Scientist Emeritus}

Robin S. (Rod) McDowell

(509) 376-3532

rs.mcdowell@pnl.gov

B.A. Haverford College 1956; Ph.D. M.I.T.

1960; Staff Member, Assistant Group Leader, and Laboratory Fellow, Los Alamos National Laboratory, 1960-1991; joined PNNL as Program Manager for Chemical Structure and Dynamics April 1991; PNNL Scientist Emeritus from June 2001. Research interests: high-resolution infrared and Raman spectroscopy applied to molecular structure and bonding; molecular dynamics and force fields; statistical mechanics; infrared analytical techniques.

\section{Postdoctoral Fellows}

Patrick Ayotte

B.Sc. Université Laval 1993; M.Sc. Université de Sherbrooke 1995; Ph.D. Yale University 1999; Postdoctoral Fellow, CS\&D, with Bruce Kay, 1999-2001.

Richard C. Bell

Ph.D. Pennsylvania State University 2000;

Postdoctoral Fellow, CS\&D, with Jim Cowin, 2000- . 
Kiran Boggavarapu

B.Sc. Osmania University 1989; M.Sc.

University of Hyderabad 1991; Ph.D.

University of Hyderabad 1997; Postdoctoral

Fellow, CS\&D, with Lai-Sheng Wang, 2002-.

You-Jun Fu

B.S. Qufu Normal University, China 1984;

M.S. Chinese Academy of Science 1987;

Ph.D. Ocean University of Qingdao, China

1994; Senior Research Fellow, CS\&D, with

Lai-Sheng Wang, 2002-.

Greg Harms

B.A./B.S. Bethel College 1992; Fulbright

Scholar/Research Assistant, Eidgennössische

Technische Hochschule, Zurich, 1997-1998;

Ph.D. University of Kansas 1998; Postdoctoral

Fellow, Leiden University (Netherlands)/

University of Linz (Austria), 1998-2000;

Postdoctoral Fellow, CS\&D, with H. Peter Lu, 2000- .

Matthias Henyk

Diploma thesis in Physics, Technical

University of Berlin 1996, Ph.D. Technical

University of Cottbus 2001; Postdoctoral

Fellow, CS\&D, with Wayne Hess, 2001- .

Dehong $\mathrm{Hu}$

B.S. Peking University 1991, M.S. 1994;

Ph.D. University of Minnesota 1999; Postdoctoral Researcher, University of Texas, Austin, 1999-2000; Postdoctoral Fellow, CS\&D, with H. Peter Lu, 2000- .

Miodrag "Mickey" Micic

B.Eng. Polytechnic Academy, New Belgrade 1991; B.Sc., B.Ed., M.Sc. and D.Sc.

University of Belgrade 1996, 1998, 2000;

$\mathrm{Ph}$.D. University of Miami 2001; Postdoctoral

Fellow, CS\&D, with H. Peter Lu, 2002-.

Serdar Ozcelik

B.S. and M.S. Ankara University, Turkey 1986, 1988; Ph.D. The City University of New York 1996; Senior Research Fellow with H. Peter Lu, 2002-.
Yung Doug Suh

B.S. Seoul National University, M.S., Ph.D. 1999; Postdoctoral Research Associate, Eidgennössische Technische Hochschule, Zurich, 1999-2001; Postdoctoral Fellow, CS\&D, with H. Peter Lu, 2001- .

Sofie X. Tan

B.S. Peking University 1991; M.S., Institute of Physics, Academia Sinica, Beijing 1994;

Ph.D. Ohio State University 2001; Postdoctoral Fellow, CS\&D, with H. Peter Lu, 2001- .

Glenn R. Teeter

B.S. Stetson University 1992; Ph.D.

University of Texas at Austin 1999; Post-

doctoral Fellow, CS\&D, with Thom Orlando

1999-2000, with Bruce Kay, 2000-2001.

Hangfu Wang

Ph.D. University of Minnesota at Twin Cities 2001; Postdoctoral Fellow, CS\&D, with Jim Cowin, 2001- .

Xin Yang

B.S. Anhui Normal University, 1989; Ph.D.

Hong Kong University of Science and

Technology, 2000; Postdoctoral Fellow,

CS\&D, with Lai-Sheng Wang, 2000- .

Hua-Jin Zhai

B.S. Wuhan University, 1990; Ph.D. Shanghai

Institute of Optics and Fine Mechanics, Chinese Academy of Sciences, 1998; Postdoctoral Fellow, CS\&D, with Lai-Sheng Wang, 2000- .

Hai-Feng Zhang

B.S. University of Science and Technology, Hefei, 1989; Ph.D., 1994; Postdoctoral Fellow, CS\&D, with Lai-Sheng Wang, 2000- .

Leyun Zhu

B.Sc. Lanzhou University 1983; M. Sc.

Northeastern University 1991, Ph.D. 1995, Research Associate 1996; NIH Postdoctoral Fellow, University of California, Berkeley, 1997-1999; Postdoctoral Fellow, CS\&D, with Peter Lu, 1999-2001. 


\section{Graduate Students}

Tiffany Coulter Chemical Engineering Dept., University of Washington, working with Wayne Hess, 2000 - .

\section{Lifeng Cui}

Physics Dept., Washington State University, working with Lai-Sheng Wang, 2002-.

\section{Jason Donev}

University of Washington, working with Steve Joyce then Scott Smith, 2000- .

\section{Xi Li}

B.S. Northwest University, Lanzhou 1986;

M.S. Chengdu University of Science and Technology 1991; Researcher, Gansu United University 1992-1995; working with LaiSheng Wang, 1996- .

Shurong (Sherry) Liu

B.S. Tianjin Normal University, 1984; M.S. 1989; Washington State University, working with Lai-Sheng Wang, 1998- .

Aimee Taylor

Oregon State University, working with Steve Barlow, Summer 2001.

Allan Tuan Chemical Engineering Dept., University of Washington, working with Wayne Hess, 2000- .

Sandra Withers-Kirby University of Central Florida, working with Wayne Hess and Ken Beck, 2000- .

\section{Undergraduate Students}

Nicholas Dupuis

Columbia University, Laboratory Co-Op

student with Wayne Hess, Summer 2001.

Brooke Holben

Washington State University, ERULF*

student with Marianne Sowa Resat, Summer 2001.

Michelle Fleck

University of Washington, Laboratory Co-Op student with Jim Cowin, Summer 2001.

Brian Long University of Washington, student with Russ Tonkyn, Summer 2001.

Nathan Moore University of Washington, ERULF* student with Jim Cowin, Summer 2001.

David Panther George Fox University, ERULF* student with Peter Lu, Summer 2002.

Brandon Peden Western Washington University, ERULF* student with Bruce Kay and Scott Smith, Summer 2001.

* ERULF - Energy Research Undergraduate Laboratory Fellowship 


\section{Publications and}

\section{Presentations from October 2000 to December 2001}

\section{Publications}

J. Akola, M. Manninen, H. Hakkinen, U. Landman, X. Li, and L. S. Wang, "Aluminum Cluster Anions: Photoelectron Spectroscopy and Ab Initio Simulations," Phys. Rev. B 62, 1321613228 (2000).

P. Ayotte, R. S. Smith, K.P. Stevenson, Z. Dohnalek, G. A. Kimmel, and B. D. Kay. "Effect of Porosity on the Adsorption, Desorption, Trapping and Release of Volatile Gases by Amorphous Solid Water," J. Geophys. ResPlanet. 106(E12):33,387-33,392 (2001).

S. E. Barlow, A. E. Taylor, and K. Swanson, "Determination of Analytic Potentials from Finite Element Computations," Int. J. Mass. Spectrom. 207, 19-29 (2001).

S. E. Barlow, R. G. Tonkyn, J. W. Hoard, and W. C. Follmer, "Cascade Processing NOx by Two-Step Discharge/Catalyst," SAE Technical Paper 2001-01-3509 (2001).

S. E. Barlow, R. G. Tonkyn, J. Hoard, and W. Follmer, "Multi-Step Discharge/Catalyst Processing of NOx in Synthetic Diesel Exhaust," SAE Technical Paper 2001-01-3510 (2001).

K. M. Beck, A. G. Joly, and W. P. Hess, "Timeresolved Femtosecond Laser Induced Desorption from Magnesium Oxide and Lithium Fluoride Single Crystals.” Sur. Sci. 451, 166-173 (2001).

K. M. Beck, A. G. Joly, and W. P. Hess, "Evidence for a Surface Exciton in $\mathrm{KBr}$ via Laser Desorption," Phys. Rev. B 63, 125423 (2001).

K. M. Beck, T. Sasaki, and N. Koshizaki, "Preparation of $\mathrm{Pt} / \mathrm{TiO} 2$ Nanocomposite Thin Films by Pulsed Laser Deposition and their Photoelectrochemical Behaviors," J. of Photoc. and Photobio. A-Chem. 145(1-2 SP ISS):11-16 (2001). (Invited paper)
A. I. Boldyrev and L. S. Wang, "Beyond Classical Stoichiometry: Experiment and Theory," J. Phys. Chem. A 105, 10759-10775 (2001). (Invited Feature Article)

J. A. Campbell, W. P. Hess, J. R. Lohman, and S. C. Goheen, "Analysis of Hanford-Related Organics Using Matrix Assisted Laser Desorption/ Ionization Time-of-Flight Mass Spectrometry, $J$. Radioanal. Nuc. Ch. 250, 247 (2001).

W. Chen, A. G. Joly, J. Z. Zhang, "Up-conversion luminescence of $\mathrm{Mn} 2+$ in $\mathrm{ZnS}: \mathrm{Mn} 2+$ nanoparticles," Phys. Rev. B, 64 (4): art. no. 041202 (2001).

B. Y. Chin, G. R. Holtom, B. D. Thrall, S. D. Colson, A. Choi, and T. H. Risby, "Using Coherent Anti-Stokes Raman Spectroscopy (CARS) to Determine Environmental Particulate Matter (EPM) Induced Macrophage (M Theta) Injury," FASEB J. 15 (4): A170-A170 Part 1 (2001).

E. Chrysostom, N. Vulpanovici, A. Masiello, J. Barber, J.W. Nibler, A. Weber, A. Maki, and T. A. Blake, "Coherent Raman and Infrared Studies of Sulfur Trioxide." J. Mol. Spect. 210, 233-239 (2001)

S. D. Colson, R. E. Gephart, V. L. Hunter, J. Janata, and L. G. Morgan, "A Risk and Outcome Based Strategy for Justifying Characterization to Resolve Tank Waste," in Science and Technology for Disposal of Radioactive Tank Wastes, W. W. Schulz and N. J. Lombardo, eds. (Plenum Publishing, New York, 1998), 77-99.

R. S. Disselkamp, M. A. Carpenter, and J. P. Cowin, "A Chamber Investigation of Nitric Acid-Soot Aerosol Chemistry at 298 K," J. Atmos. Chem. 37, 113-123 (2000).

Z. Dohnálek, G. A. Kimmel, S. A. Joyce, P. Ayotte, R. S. Smith, and B. D. Kay, "Physisorption of CO on the $\mathrm{MgO}(100)$ Surface," J. Phys. Chem. B 105, 3747-3751 (2001). 
A. R. Felmy, Z. Wang, D. A. Dixon, A. G. Joly, J. R. Rustad, and M. J. Mason. "The Aqueous Complexation of Eu(III) with Organic Chelates at High Base Concentration: Molecular and Thermodynamic Modeling Results." Chapter 5 in Nuclear Site Remediation: First Accomplishments of the Environmental Management Science Program, Vol. 778, ed. P. G. Eller, W. R. Heinemann. American Chemical Society Symposium Series, Washington DC. (2001) pp. 63-82

G. D. Geske, A. I. Boldyrev, X. Li, and L.-S. Wang, "On the Origin of Planarity in $\mathrm{Al}_{5}{ }^{-}$ and $\mathrm{Al}_{5}$ Clusters: The Importance of a FourCenter Peripheral Bond," J. Chem. Phys. 113, 5130-5133 (2000).

M. Gutowski, P. Skurski, X. Li, and L.-S. Wang, "( $\mathrm{MgO})_{\mathrm{n}}^{-}(n=1-5)$ Clusters: Multipole-Bound Anions and Photodetachment Spectroscopy," Phys. Rev. Lett. 85, 3145-3148 (2000).

G. L. Gutsev, P. Jena, H. J. Zhai, and L.-S. Wang, "Electronic Structure of Chromium Oxides, $\mathrm{CrO}_{\mathrm{n}}$ " and $\mathrm{CrO}_{\mathrm{n}}(\mathrm{n}=1-5)$ from Photoelectron Spectroscopy and Density Functional Theory Calculations," J. Chem. Phys. 115, 7935-7944 (2001). $10 / 9 / 00$

W. P. Hess, A. G. Joly, D. P. Gerrity, K. M. Beck, P. V. Sushko, and A. L. Shluger, "Selective Laser Desorption of Ionic Surfaces: Resonant Surface Excitation of KBr," J. Chem. Phys. 115, 9463 (2001).

J. Hoard, P. Laing, M. L. Balmer, and R. G. Tonkyn, "Comparison of Plasma-Catalyst and Active Lean NOx Catalyst for Diesel NOx Reduction," SAE Tech. Paper 2001-01-2895 (2000).

G. R. Holtom, B. D. Thrall, B.-Y. Chin, H. S. Wiley, and S. D. Colson, "Achieving Molecular Selectivity In Imaging Using Multiphoton Raman Spectroscopy Techniques," Traffic 2 (11):781 (2001). (Invited paper)

Th. Huser, L. Novotny, Th. Lacoste, R. Eckert, and H. Heinzelmann, "Observation and Analysis of Near-Field Optical Diffraction," J. Opt. Soc. Am. A, 16 (1): 141-148 (1999).
A. G. Joly, W. Chen, J. Roark, and J. Z. Zhang, "Temperature Dependence of Up-Conversion Luminescence and Photoluminescence of $\mathrm{Mn} 2+$ in Zns: Mn2+ Nanoparticles," J Nanosci. Nanotech. 1 (3):295-301 (2001).

S. Kato, T. T. Dang, S. E. Barlow, C. H. DePuy, and V. M. Bierbaum, "Gas-Phase Association Reactions of Hydroxide, Methoxide, Ethoxide and Their Deuterated Analogs," Int. J. Mass Spectrom. and Ion Processes, 196, 625-638 Sp. Iss. (2000).

J. F. Kelly, R. M. Williams, T. L. Myers, M. S. Taubman, D. M. Sheen, and S. W. Sharpe, "Chemical Sensing with Quantum Cascade Lasers," LEOS 2000_IEEE Annual Meeting Conference Proceedings, 191-192 (2000).

J. H. Kim, X. Li, L.-S. Wang, H. L. de Clercq, C. A. Fancher, O. C. Thomas, and K. H. Bowen, "Vibrationally Resolved Photoelectron Spectroscopy of $\mathrm{MgO}^{-}$and $\mathrm{ZnO}^{-}$and the Low-Lying Electronic States of $\mathrm{MgO}$ and $\mathrm{ZnO}$," J. Phys. Chem. A 105, 5709-5718 (2001).

G. A. Kimmel, K. P. Stevenson, Z. Dohnálek, R. S. Smith, and B. D. Kay, "Control of Amorphous Solid Water Morphology Using Molecular Beams. I. Experimental Results," J. Chem. Phys. 114, 5284-5294 (2001).

G. A. Kimmel, Z. Dohnálek, K. P. Stevenson, R. S. Smith, and B. D. Kay, "Control of Amorphous Solid Water Morphology Using Molecular Beams. II. Ballistic Deposition Simulations," $J$. Chem. Phys. 114, 5295-5303 (2001).

A. E. Kuznetsov, A. I. Boldyrev, X. Li, and L.-S. Wang, "On the Aromaticity of Square Planar $\mathrm{Ga}_{4}{ }^{2-}$ and $\mathrm{In}_{4}{ }^{2-}$ in Gaseous $\mathrm{NaGa}_{4}{ }^{-}$and $\mathrm{NaIn}_{4}{ }^{-}$ Clusters," J. Am. Chem. Soc. 123, 8825-8831 (2001).

A. E. Kuznetsov, J. D. Corbett, L.-S. Wang, and A. I. Boldyrev, "Aromatic Mercury Clusters in Ancient Amalgams," Angew. Chem. Int. Edit. 40, 3369-3372 (2001).

A. Laskin and J. P. Cowin, "Automated SingleParticle SEM/EDX Analysis of Submicrometer Particles down to $0.1 \mu \mathrm{m}$," Anal. Chem. 73, 10231029 (2001). 
X. Li, A. E. Kuznetsov, H. F. Zhang, A. I. Boldyrev, and L.-S. Wang, "Observation of All-Metal Aromatic Molecules," Science 291, 859-861 (2001).

X. Li, H. F. Zhang, L.-S. Wang, G. D. Geske, and A. I. Boldyrev, "Pentaatomic Tetracoordinate Planar Carbon, $\left[\mathrm{CAl}_{4}\right]^{2-}$ : A New Structural Unit and Its Salt Complexes," Angew. Chem. Int. Ed. Engl. 39, 3630-3633 (2000).

A. G. Maki, T. A. Blake, R. L. Sams, N. Vulpanovici H. F. Zhang, L.-S. Wang, A.E. Kuznetsov, N. A. Cannon, and A. I. Boldyrev, "Experimental and Theoretical Observations of Artomaticity in Heterocyclic $\mathrm{XAl}_{3}^{-}(\mathrm{X}=\mathrm{Si}, \mathrm{Ge}, \mathrm{Sn}, \mathrm{Pb})$ Systems," Angew. Chem. Int. Edit. 40, 1867-1870 (2001).

J. C. Linehan, C. R. Yonker, R. S. Addleman, S.Autrey, J. T. Bays, T. E. Bitterwolf, and J. L. Daschbach, "Solvent Study of the Kinetics of Molybdenum Radical Self-Termination," Organometallics 20(3):401-407 (2001).

S. R. Liu, H. J. Zhai, and L.-S. Wang, "Electronic and Structural Evolution of $\mathrm{Co}_{\mathrm{n}}$ Clusters ( $n=1-108)$ by Photoelectron Spectroscopy," Phys. Rev. B. 64, 153402-1-4 (2001).

H. Lu, L. M. Iakoucheva, and E. J. Ackerman, "Single-Molecule Conformational Dynamics of Fluctuating Noncovalent DNA - Protein Interactions in a DNA Damage Recognition," $J$. of the $A C S$, 123(37):9184-9185 (2001).

K. Luong, T. G. Clements, M. S. Resat, and R. E. Continetti, "Energetics and Dissociative Photodetachment Dynamics of Superoxide-Water clusters: $\mathrm{O}^{2-}\left(\mathrm{H}_{2} \mathrm{O}\right)_{n}, n=1-6, " J$. Chem. Phys. 114, 3449 (2001).

A. G. Maki, T. A. Blake, R. L. Sams, N. Vulpanovici, J. B. Barber, E. Chrsostom, A.Masiello, J. W. Nibler, and A. Weber, "High Resolution Infrared Spectra of the v2, v3, v4 and $2 v 3$ Band of ${ }^{32} \mathrm{~S}^{16} \mathrm{O}_{3}$," J. Mol. Spect. 210, 240-249 (2001).

T. B. McCord, T. M. Orlando, G. Teeter, G. B. Hansen, M. T. Sieger, N. G. Petrik, and L. Van Keulen, "Thermal and Radiation Stability of the Hydrated Salt Minerals Epsomite, Mirabilite, and Natron under Europa
Environmental Conditions," J. Geophys. Res.Planets 106, 3311-3319 (2001).

P. R. McCurdy, E. R. Vorpagel, and W. P. Hess, "Ultraviolet Photochemistry of Hydrogen-Bonded $\mathrm{HBr}$-Acetone Complexes in Argon Matrices," J. Chem. Phys. A 114, 169-178 (2001).

A. Panov, M. L. Balmer, S. Yoon, A. Kolwaite, R. G. Tonkyn, and S. E. Barlow, "Effect of Stimulated Diesel Exhaust Gas Composition and Catalyst Temperature on NOx Reduction Behavior of Alumina and Zeolite Catalysts in Combination with Non-Thermal Plasmas," SAE Technical Paper 2001-01-2964 (2000).

A. G. Panov, R. G. Tonkyn, C. H. Peden, J. W. Hoard, and M. L. Balmer, "Selective Reduction of NOx in Oxygen Rich Environment with Plasma-Assisted Catalysis: Role of Plasma and Reactive Intermediates," SAE Technical Paper 2001-01-3513 (2001).

N. G. Petrik, A. B. Alexandrov, and A. I. Vall, "Interfacial Energy Transfer during Gamma Radiolysis of Water on the Surface of $\mathrm{ZrO} 2$ and Some Other Oxides," J. Phys. Chem. B 105(25):5935-5944, (2001).

R. J. Reeder, M. Nugent, C. D. Tait, D. E. Morris, S. M. Heald, K. M. Beck, W. P. Hess, and A. Lanzirotti, "Coprecipitation of Uranium(VI) with Calcite: XAFS, Micro-XAS, and Luminescence Characterization," Geochim. Cosmochim. Acta 65, 3491 (2001).

C. L. Schiller, S. Locquiao, T. J. Johnson, and G. W. Harris, "Atmospheric Measurements of HONO by Tunable Diode Laser Absorption Spectroscopy," J. Atm. Chem. 40 (3): 275-293 (2001).

M. D. Tinkle and S. E. Barlow, "Image Charge Forces Inside Conducting Boundaries," J. Appl. Phys. 90(3):1612 (2001).

R. Tonkyn, S. Yoon, S. E. Barlow, A. Panov, A. Kolwaite, and M. L. Balmer, "Lean NOx Reduction in Two Stages: Non-Thermal Plasma Followed by Heterogeneous Catalysis," SAE Technical Paper 2001-01-2896 (2000). 
L.-S. Wang, X. Li, and H. F. Zhang, "Probing the Electronic Structure of Iron Clusters Using Photoelectron Spectroscopy," Chem. Phys. 262, 53-63 (2000).

L.-S. Wang, "Photodetachment of MultiplyCharged Anions," Comments on Mod. Phys. 2 (5): D207-D221 (2001).

X. B. Wang, J. B. Nicholas, and L.-S. Wang, "Electronic Instability of Isolated $\mathrm{SO}_{4}{ }^{2-}$ and its Solvation Stabilization," J. Chem. Phys. 113, 10837-10840 (2000).

X. B. Wang and L.-S. Wang, "The Electronic Structure and Electron Affinities of Higher Chlorine Oxide Radicals $\mathrm{ClO}_{x}(x=2-4)$ from Photoelectron Spectroscopy of $\mathrm{ClO}_{x}^{-}$Anions," J. Chem. Phys. 113, 10928-10933 (2000).

X.-B. Wang, L.-S. Wang, R. Brown, P. Schwerdtfeger, D. Schroder, and H. Schwarz, "The Electronic Structure of $\mathrm{CuCl}_{2}$ and $\mathrm{CuBr}_{2}$ from Anion Photoelectron Spectroscopy and $\mathrm{Ab}$ Initio Calculations," J. Chem. Phys. 114, 73887395 (2001).

X. B. Wang, X. Yang, J. B. Nicholas, and L.-S. Wang, "Bulk-Like Features in the Photoemission Spectra of Hydrated Doubly-Charged Anion Clusters," Science 294, 1322-1325 (2001).

X. B. Wang, E. R. Vorpagel, X. Yang, and L.-S. Wang, "Experimental and Theoretical Investigations of the Stability, Energetics, and Structures of $\mathrm{H}_{2} \mathrm{PO}_{4}^{-}, \mathrm{H}_{2} \mathrm{P}_{2} \mathrm{O}_{7}{ }^{2-}$, and $\mathrm{H}_{3} \mathrm{P}_{3} \mathrm{O}_{10}{ }^{2-}$ in the Gas Phase," J. Phys. Chem. A 105, 1046810474 (2001).

Z. Wang, D. M. Friedrich, C. C. Ainsworth, S. L. Hemmer, A. G. Joly, and M. R. Beversluis, "Ground-State Proton Transfer Tautomer of Al(III)-Salicylate Complexes in Ethanol Solution," J. Phys. Chem. A 105, 942-950 (2001).
Z. Wang, D. Friedrich, M. R. Beversluis, S. L.Hemmer, A. G. Joly, M. H. Huesemann, M. J. Truex, R. G. Riley, C. J. Thompson, and B. M. Peyton, "A Fluorescence Spectroscopic Study of Phenanthrene Sorbed on Porous Silica," Environ. Sci.Tech. 35(13):2710-2716 (2001).

Z. Wang, S. L. Hemmer, D. Friedrich, and A. G. Joly, "Anthracene as the Origin of the RedShifted Emission from Commercial Zone-Refined Phenanthrene Sorbed on Mineral Surfaces," J. Phys. Chem. A 105(25):6020-6023 (2001).

R. A. Wind, K. R. Minard, G. R. Holtom, P. D. Majors, E. J. Ackerman, S. D. Colson, D. G. Cory, D. S. Daly, P. D. Ellis, N. F. Metting, C. I. Parkinson, J. M. Price, and X. W. Tang, "An Integrated Confocal and Magnetic Resonance Microscope for Cellular Research," J. Mag. Res. 147, 371-377 (2000).

K. Wu, M. J. Iedema, G. K. Schenter, and J. P. Cowin, "Sculpting the Oil-Water Interface to Probe Ion Solvation," J. Phys. Chem. B 105, 2483-2498 (2001). (Invited Feature Article.)

X. Yang, X. B. Wang, and L.-S. Wang, "Photodetachment of $\mathrm{F}^{-}\left(\mathrm{H}_{2} \mathrm{O}\right)_{\mathrm{n}}(n=1$ to 4$)$ : Observation of Charge-Transfer States $\left[\mathrm{F}^{-}\left(\mathrm{H}_{2} \mathrm{O}\right)_{\mathrm{n}}{ }^{+}\right]$and the Transition State of $\mathrm{F}+\mathrm{H}_{2} \mathrm{O}$ Hydrogen Abstraction Reaction," J. Chem. Phys. 115, 2889-2892 (2001).

H. J. Zhai, S. Liu, X. Li, and L.-S. Wang, "Photoelectron Spectroscopy of Mono-Niobium Carbide Clusters $\mathrm{NbC}_{\mathrm{n}}^{-}(n=2-7)$ : Evidence for a Cyclic to Linear Structural Transition," J. Chem. Phys. 115, 5170-5178 (2001). 


\section{In Press}

P. Ayotte, S. B. Nielsen, G. H. Weddle, M. A. Johnson, and S. S. Xantheas, "Spectroscopic Observation of Ion-Induced Water Dimer Desorption in the $\mathrm{X}-\left(\mathrm{H}_{2} \mathrm{O}\right)_{2}(\mathrm{X}=\mathrm{F}, \mathrm{Cl}, \mathrm{Br}, \mathrm{I})$ Clusters," J. Phys. Chem. A, in press.

S. E. Barlow, A. E. Taylor, and K. R. Swanson, "Analytic Potentials for Realistic Electrodes," Non-Neutral Plasma Physics IV, in press.

K. M. Beck, A. G. Joly, W. P. Hess, D. P. Gerrity, N. F. Dupuis, P. V. Sushko, and A. L. Shluger "Transient Center Photodecomposition in Potassium Bromide" Appl. Surf. Sci., in press.

L. P. Cramer, S. C. Lanford, W. P. Hess, and J. T. Dickinson. "Interactions of Wide Bandgap Single Crystals with 157-nm Excimer Laser Radiation I. $\mathrm{NaNO}_{3}$." Appl. Surf. Sci., in press.

V. M. Devi, D. C. Benner, L. R. Brown, M. A. H. Smith, C. P. Rinsland, R. L. Sams, and S. W. Sharpe, "Multispectrum Analysis of Selfand $\mathrm{N}_{2}$-Broadening, Shifting and Line Mixing Coefficients in the $v_{6}$ Band of ${ }^{12} \mathrm{CH}_{3} \mathrm{D}$," J. Quant. Spect.Rad. Trans., in press.

Z. Dohnalek, G. A. Kimmel, D. E. McCready, J. S. Young, A. Dohnalkova, R. S. Smith, and B. D. Kay, "Structural and Chemical Characterization of Aligned Crystalline Nanoporous $\mathrm{MgO}$ Films Grown via Reactive Ballistic Deposition," J. Phys. Chem B., in press.

E. J. Elzinga, R. J. Reeder, S. H. Withers, R. E. Peale, R. A. Mason, K. M. Beck, and W. P Hess, "XAFS Study of Rare Earth Element Coordination in Calcite." Geochim. Cosmochim. Acta, in press.

G. S. Harms, G. Orr, M. Montal, B. D. Thrall, S. D. Colson, and H. P. Lu, "Combined PatchClamp Recording and Single-Molecule Imaging Microscopy Study of Single-Molecule Ion Channel Dynamics," Biophys. J., in press.

W. P. Hess, A. G. Joly, D. P. Gerrity, K. M. Beck, P. V. Sushko, and A. L. Shluger, "Control of Laser Desorption Using Tunable Single Pulses and Pulse Pairs." J. Chem. Phys., in press.
W. P. Hess, A. G. Joly, K. M. Beck, D. P. Gerrity, P. V. Sushko, and A. L. Shluger, "Solid-state Halogen Atom Source for Chemical Dynamics and Etching." Appl. Phys. Lett., in press.

G. R. Holtom, B. Thrall, T. Weber, L. Zhu, D. Hopkins, C. Parkinson, S. Colson, J. Price, B. Y. Chin, M. E. Choi, and T. Risby, "Application of Coherent Anti-Stokes Raman Scattering (CARS) to Imaging Mammalian Cells: A Means for Gaining Molecular Selectivity in Multiphoton Imaging," Proc. SPIE, in press.

D. H. Hu, Y. Chen, E. R. Vorpagel, and H. P. Lu, "Single-Molecule Conformational Mode-Specific Dynamics Under Enzymatic Reaction,"

Biophys. J., in press

A. G. Joly, W. P. Hess, K. M. Beck, J. T. Dickinson, P. V. Sushko, and A. L. Shluger, "Femtosecond Time-Resolved Photostimulated Desorption from Ionic Crystals," Appl. Surf. Sci., in press.

A. Laskin and J. P. Cowin, "On Deposition Efficiency of Point-to-Plate Electrostatic Precipitator," J. Aerosol Sci., in press

X. Li and L.-S. Wang, "Experimental Search and Characterization of Icosahedral Clusters: A112X$(\mathrm{X}=\mathrm{C}, \mathrm{Ge}, \mathrm{Sn}, \mathrm{Pb})$, , Phys. Rev, B., in press.

H. P. Lu and Y. Chen, "Single-Molecule Spectroscopy Study of the Mechanisms and Dynamics of Non-Covalent and Covalent Molecular Interactions," Biophys. J., in press.

H. P. Lu and X. S. Xie, "Single-Molecule Enzymatic Dynamics: Static Disorder and Dynamic Disorder," J. Lumin., in press.

P. R. McCurdy, W. P. Hess, and S. Xantheas, "Nitric Acid-Water Complexes: Theoretical Calculations and Comparison to Experiment." J. Phys. Chem. In press

V. B. Mikheev, P. M. Irving, N. S. Laulainen, S. E. Barlow, and V. V. Pervukhin, "Laboratory Measurement of Water Nucleation Using Laminar Flow Tube Reactor," J. Chem. Phys. in press.

G. Orr, G. S. Harms, B. D. Thrall, M. Montal, S. D. Colson, and H. P. Lu, "Probing SingleMolecule Ligand-Channel Interaction Dynamics in a Living Cell," Biophys J., in press. 
G. Orr, M. Montal, B. D. Thrall, S. D. Colson, and H. P. Lu, "Single Channel Patch-Clamp Recording Coupled with Linear and Nonlinear Confocal Scanning Fluorescence Spectroscopy: Towards the Simultaneous Probing of Single-Ion Channel Conformational Changes and Channel Kinetics, Biophys. J., in press.

M. R. Papantonakis, J. Kim, W. P. Hess, and R. F. Haglund, Jr., "What Do MALDI Mass Spectra Reveal About Ionization Mechanisms?" $J$. Mass Spectrom. in press.

R. S. Smith, Z. Dohnálek, G. A. Kimmel, K. P. Stevenson, and B. D. Kay, "The Relationship between the Self-Diffusivity of Supercooled and Amorphous Solid Water," in Liquid Dynamics: Into the New Millennium, ACS Symposium Series, in press.

R. Tonkyn, S. E. Barlow, S. Yoon, A. Panov, A. Ebeling, and M. L. Balmer, "Lean NOx Reduction by Plasma Assisted Catalysis," Proc. DEER 2000, in press.

L. S. Wang, "Clusters and Nanoscale Structures," Encyclopedia of Chemical Physics and Physical Chemistry, ed. by J. H. Moore and N. D. Spencer (IOP Publishing Inc., Philadelphia, 2000), in press.

X. B. Wang, X. Yang, L.-S. Wang, and

J. B. Nicholas, "Photodetachment and Theoretical Study of Free and Water-Solvated Nitrate Anions, NO3-(H2O)(n) (n=0-6)," J. Chem. Phys. in press.

R. A. Wind, P. D. Majors, K. R. Minard, E. J. Ackerman, D. S. Daly, G. R. Holtom, B. D. Thrall, and T. J. Weber, "Combined Confocal and Magnetic Resonance Microscopy," Appl. Mag. Res. in press.

H.-F. Zhang, A. C. Dohnalkova, C. M. Wang, J. S. Young, E. C. Buck, and L.-S. Wang, "Lithium-Assisted Self-Assembly of Aluminum Carbide Nanowires and Nanoribbons," Nano Lett., in press.

\section{Presentations}

Presenter underlined; $*$ = invited talk.

P. Ayotte, J. L. Daschbach, G. A. Kimmel, A. Billic, H. Jonsson, and B. D. Kay, "Molecular Beam and Classical Trajectory Studies of Helium Absorption and Diffusion in Ice," Poster at the Gordon Research Conference on Dynamics at Surfaces, Andover, NH, August 2001.

P. Ayotte, J. L. Daschbach, G. A. Kimmel, A. Billic, H. Jonsson, and B. D. Kay, "Molecular Beam and Classical Trajectory Studies of Helium Absorption and Diffusion in Ice," American Physical Society, Seattle, WA, Mar. 16, 2001.

P. Ayotte, Z. Dohnálek, G. A. Kimmel, S. Smith, and B. D. Kay, "Using Nanoscale Amorphous Films to Study Mixing, Transport and Phase Separation in Deeply Supercooled, Metastable Binary Solutions of Methanol, Ethanol, and Water," National Meeting, American Vacuum Society, San Francisco, CA, Oct. 30 - Nov. 1, 2001.

P. Ayotte, Z. Dohnálek, G. A. Kimmel, S. Smith, and B. D. Kay, "Using Nanoscale Amorphous Films to Study Mixing, Transport and Phase Separation in Deeply Supercooled, Metastable Binary Solutions of Methanol, Ethanol, and Water," Poster at the Gordon Research Conference on Dynamics at Surfaces, Andover, NH, August 2001.

S. E. Barlow, A. E. Taylor, and K. Swanson, "Determination of Analytic Potentials from Finite Element Computations," Workshop on NonNeutral Plasmas, San Diego, CA, July 31, 2001.

K. M. Beck, "Toxic Metal Uptake by Carbonates in Soil," Coastal Carolina University, Conway S.C, Oct. 19, 2000.*

K. M. Beck, A. G. Joly, W. P. Hess, D. Gerrity, P. V. Sushko, and A. L. Shluger, "Transient Center Photodecomposition in Potassium Bromide," Conference on Laser Ablation 2001 (COLA), Tsukuba, Japan, Oct. 1-5, 2001.

R. C. Bell, K. Wu, M. J. Iedema, and J. P. Cowin, "Nano-Scale Effects on the Fluidity of Organic Films," American Physical Society, Seattle, WA, Mar. 15, 2001. 
R. C. Bell, K. Wu, M. J. Iedema, and J. P. Cowin, "Nano-Scale Effects on the Interfacial Fluidity of Organic Films," Northwest Regional Meeting, American Chemical Society, Seattle, WA, June 17, 2001.

A. Bilic, H. Jonsson, and B. D. Kay, "A Molecular Dynamics Study of the Helium Absorption by Amorphous and Crystalline Ice," American Physical Society, Seattle, WA, Mar. 16, 2001.

T. A. Blake, R. L. Sams, A. Maki, T. Masiello, J. Barber, N. Vulpanovici, E.T.H. Chrysostom, J. W. Nibler, and A. Weber, "Observation and Analysis of the $2 v_{3}$ Band of ${ }^{32} \mathrm{~S}^{16} \mathrm{O}_{3}$, , 56th International Symposium on Molecular Spectroscopy, Columbus, OH, June 11-15, 2001.

L. R. Brown, R. L. Sams, I. Kleiner, and C. Cottaz, "Line Intensities of Phosphine in the $10 \mu \mathrm{m}$ Region for Planetary Spectra Analysis," 56th International Symposium on Molecular Spectroscopy, Columbus, OH, June 11-15, 2001.

E. T. H. Chrysostom, T. Masiello, J. Barber, N. Vulpanovici, J. W. Nibler, A. Weber, A. Maki, T. A. Blake, and R. L. Sams, "CARS Observation and Analysis of the $v_{1}$ Band of ${ }^{32} \mathrm{~S}^{16} \mathrm{O}_{3}$," 56th International Symposium on Molecular Spectroscopy, Columbus, OH, June 11-15, 2001.

L. Cognet G. S. Harms, G. Blab, P. H. Lommerse, and T. Schmidt, "Simultaneous Dual Color, Dual Polarization Detection of Single Molecules," Annual Meeting, American Biophysical Society, Boston, MA, Feb. 18, 2001.

S. D. Colson, "Live Cell Multi-Functional Imaging," NORM 2001, Seattle, WA, May 14, 2001.*

J. P. Cowin and M. J. Iedema, "Mapping the Solvation Potential at the Oil/Water Interface," Department of Chemistry, University of Wisconsin, Madison, WI, Oct. 17, 2000.*

J. P. Cowin and M. J. Iedema, "Ion Transport in Organics and Their Solvation at Aqueous Interfaces," Chemical Sciences, Argonne National Laboratory, Argonne, IL, Oct. 18, 2000.*
J. P. Cowin, M. J. Iedema, and A. Laskin, "TimeResolved Field Collector for Aerosols," Annual Symposium, American Association of Aerosol Researchers, St. Louis, MO, Nov. 6-10, 2000 (poster).

J. P. Cowin, M. J. Iedema, A. Laskin, S. Joyce, and D. Gaspar, "Surface Sensitive Analysis of Atmospheric Aerosols," Annual Symposium, American Association of Aerosol Researchers, St. Louis, Mo, Nov. 6-10, 2000 (poster).

J. P. Cowin and A. Laskin, "Single Particle Analysis of Air-borne Particles from Houston," EPA PM Center, University of Washington, Seattle, WA, Jan. 11, 2001.*

J. P. Cowin, A. Laskin, and M. Iedema, "Single Particle Analysis of Houston Area Atmospheric Aerosols," Environmental Health Dept., University of Washington, Seattle, WA, Jan. 11, 2001.

J. L. Daschbach, B. M. Peden, G. R. Teeter, Glenn, R. S. Smith, B. D. Kay, "A Molecular Beam Study of Water Adsorption, Desorption, and Clustering on Pt (111)," Poster at the Gordon Research Conference on Dynamics at Surfaces, Andover, NH, August 2001.

J. L. Daschbach, B. M. Peden, G. R. Teeter, R. S. Smith, B. D. Kay, "A Molecular Beam Study of Water Adsorption, Desorption, and Clustering on Pt(111)," AVS Northwest Chapter Meeting, Forest Grove, OR, September 2001.

J. L. Daschbach, B. M. Peden, G. R. Teeter, R. S. Smith, B. D. Kay, "A Molecular Beam Study of Water Adsorption, Desorption, and Clustering on Pt(111)," 48th International Symposium of American Vacuum Society, San Francisco, CA, October 2001.

J. L. Daschbach, P. Ayotte, G. A. Kimmel, Z. Dohnálek, S. Smith, and B. D. Kay, “A Molecular Beam Study of Helium Absorption and Diffusion in Amorphous and Crystalline Ice," National Meeting, American Vacuum Society, San Francisco, CA, Oct. 30 - Nov. 1, 2001. 


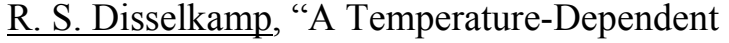
Ozonolysis Study of Ethene and Propene," American Geophysical Union, San Francisco, Dec. 15-19, 2000.

Z. Dohnálek, G. A. Kimmel, P. Ayotte, R. S. Smith, S. A. Joyce, and B. D. Kay, "Defect Characterization on $\mathrm{MgO}(100)$ Using Adsorption of Small Molecules," 47th International Symposium, American Vacuum Society, Boston, MA, Oct. 1-5, 2000.

Z. Dohnálek, G. A. Kimmel, P. Ayotte, R. S. Smith, S. A. Joyce, and B. D. Kay, "Defect Characterization on $\mathrm{MgO}(100)$ Using Adsorption of Small Molecules," 2000 National Laboratory Catalysis Research Conference, Argonne IL., Oct. 12-13, 2000.

Z. Dohnálek, D. E. McCready, J. S. Young, A. Dohnalkova, G. A. Kimmel, S. A. Joyce, P. Ayotte, S. Smith, and B. D. Kay, "Characterization of Surface Defects on Flat and Porous MgO Surfaces," 221 st Annual Meeting, American Chemical Society, San Diego, CA, Apr. 1-5, 2001.*

Z. Dohnálek, G. A. Kimmel, D. E. McCready, J. S. Young, A. Dohnálková, R. S. Smith, and B.D. Kay, "Synthesis and Characterization of Nanoporous Thin MgO Films using Molecular Beams," The Workshop on Exploiting Opportunities in Nanoscience and Nanotechnology, Richland, WA, Feb. 2001.

Z. Dohnálek, "From Ice to Oxides: Synthesis and Characterization of Nanoporous Thin Films Using Molecular Beams," Nanotechnology Seminar Series at University of Washington, Seattle, WA, May 2001.*

Z. Dohnálek, G. A. Kimmel, D. E. McCready, J. S. Young, A. Dohnálková, R. S. Smith, and B. D. Kay, "Synthesis and Characterization of Nanoporous Thin MgO Films using Molecular Beams," Dynamics at Surfaces, Gordon Research Conference, Andover, NH, August 2001.

Z. Dohnálek, G. A. Kimmel, P. Ayotte, R. S. Smith, S. A. Joyce, and B. D. Kay, "Adsorption - Desorption Studies on $\mathrm{MgO}(100)$ Surfaces," Dynamics at Surfaces, Gordon Research Conference, Andover, NH, August 2001.
Z. Dohnálek, "From Ice to Oxides: Synthesis and Characterization of Nanoporous Thin Films Using Molecular Beams," Seminar at University of Missouri-Rolla, Rolla, MO, September 2001.*

Z. Dohnálek, D. E. McCready, J. S. Young, A. Donalkova, G. A. Kimmel, S. Smith, and B. D. Kay, "Characterization of Surface Defects on Flat and Porous MgO Surfaces," National Meeting, American Vacuum Society, San Francisco, CA, Oct. 30 - Nov. 1, 2001.

J. M. Donev, S. C. Fain Jr., and S. A. Joyce, "Atomic Force Microscopy (AFM) of Ice VaporDeposited on $\mathrm{Au}(111)$ at $100 \mathrm{~K}$," Third Annual Meeting, Northwest Section, American Physical Society, Seattle, WA, May 25, 2001.

J. M. Donev, S. A. Joyce, and S. C. Fain, "Morphology of Vapor-Deposited Ice on $\mathrm{Au}(111)$ at Low Temperatures," Scanning Tunneling Microscopy/Spectroscopy and Related Techniques, Vancouver BC, July 17, 2001.

S. C. Fain, J. M. Donev, and S. A. Joyce, "Morphology of Vapor-Deposited Ice at Low Temperatures by Atomic Force Microscopy," 48th International Meeting, American Vacuum Society, San Francisco, CA, Oct. 15, 2001.

G. S. Harms, P. H. Lommerse, H. C. Kahr, and N. Soldatov, "The Aggregation of Individual eYPF-Labeled L-Type Calcium Ion Channels in Living Cell Membranes," Annual Meeting, American Biophysical Society, Boston, MA, Feb. 18, 2001.

G. S. Harms, P. H. Lommerse, and N. Soldatov, "The Autofluorescent Proteins in Single Molecule Research: Applications to Cells," Annual Meeting, American Biophysical Society, Boston, MA, Feb. 18, 2001.

J. G. Hering, D. E. Giammar, Z. Wang, and A. G. Joly, "Investigation of the structure of surface complexes using a fluorescent probe," Division of Environmental Chemistry, 222 ${ }^{\text {nd }}$ American Chemical Society (ACS) National Meeting, Chicago, IL, Aug. 26-30 (2001).

W. P. Hess, A. G. Joly, K. M. Beck, and D. P. Gerrity, "Vaporization of Solids using High Power Pulsed Lasers," Reed College, Portland, OR, October 2000.* 
W. P. Hess, A. G. Joly, K. M. Beck, D. P. Gerrity, P. Sushko, and A. L. Shluger, "Photostimulated Desorption from Crystalline Solids: Laser Control of Product States and Femtosecond TimeResolved Studies," University of Central Florida, Orlando, FL, October 2000.*

W. P. Hess, A. G. Joly, K. M. Beck, D. P. Gerrity, P. Sushko, and A. L. Shluger, "Control of Laser Induced Reactions in Solids Using Femtosecond and Nanosecond Pulses," Conference on Laser Ablation 2001 (COLA), Tsukuba, Japan, Oct. 1-5, 2001.

W. P. Hess, A. G. Joly, K. M. Beck, D. P. Gerrity, P. Sushko, and A. L. Shluger, "Photostimulated Desorption from Crystalline Solids: Laser Control of Product States and Femtosecond and Nanosecond Lasers," University College, London, Nov. 16,2000 * $^{*}$

W. P. Hess, A. G. Joly, K. M. Beck, D. P. Gerrity, P. Sushko, and A. L. Shluger, "Laser Induced Reactions in Solids: Femtosecond Time-Resolved Studies and Product State Control," Oregon State University, Corvallis, November 2000.*

W. P. Hess, A. G. Joly, D. P. Gerrity, K. M. Beck, P. Sushko, and A. Shluger, "Photostimulated Desorption from Crystalline Solids: Laser Control of Product States and Femtosecond TimeResolved Studies," Symposium on Femtosecond Materials Science and Technology, Materials Research Society, San Francisco, CA, April 18, 2001.*

W.P. Hess, A.G. Joly, D.P. Gerrity, K.M. Beck, P.V. Sushko, and A.L. Shluger, "Laser Desorption of Ground State Neutrals from Crystalline KBr," Gordon Research Conference on Dynamics at Surfaces, Andover, NH, August 2001.

W.P. Hess, A.G. Joly, D.P. Gerrity, K.M. Beck, P.V. Sushko, and A.L. Shluger, "Laser Control of a Solid-State Reaction: Photostimulated Desorption from Potassium Bromide," Gordon Research Conference on Dynamics at Surfaces, Andover, NH, August 2001.
G. R. Holtom and B. D. Thrall, "Application of Coherent Anti-Stokes Raman Scattering (CARS) to Imaging Mammalian Cells: A Means for Gaining Molecular Selectivity in Multiphoton Imaging," SPIE Meeting, San Jose, CA, Jan. 23,2001.

G. R. Holtom, B. D. Thrall, B. Y. Chin, and T. H. Risby, "The Application of Nonlinear Raman Spectroscopy to Provide Molecular Specificity in 3-D Biological Imaging," CLEOQELS 2001, Baltimore, MD, Mar. 8, 2001.

G. R. Holtom, B. D. Thrall, T. J. Weber, L. Zhu, D. F. Hopkins, C. I. Parkinson, S. D. Colson, J. M. Price, B. Y. Chin, A. Choi, and T. H. Risby, "Application of Coherent Anti-Stokes Raman Scattering (CARS) to Imaging Mammalian Cells: A Means for Gaining Molecular Selectivity in Multiphoton Imaging," SPIE Bios/Photonics West, San Jose CA, Jan. 23, 2001.

A. G. Joly, W. P. Hess, K. M. Beck, J. T. Dickinson, P. Sushko, and A. L. Shluger, "Femtosecond Time-Resolved Photostimulated Desorption from Ionic Crystals," European Materials Research Society, Strasbourg, France, June 7, 2001.

A. G. Joly, W. P. Hess, D. P. Gerrity, K. M. Beck, P. Sushko, and A. L. Shluger, "Laser Control of a Solid State Reaction: Photostimulated Desorption from Potassium Bromide," European Materials Research Society, Strasbourg, France, June 7,2001.

S. A. Joyce, J. T. Atherley, and J.P. Cowin, "SEM Studies of the Hydration of Inorganic Salts," 47th International Symposium, American Vacuum Society, Boston, MA, Oct. 1-5, 2000.

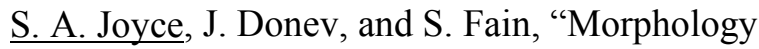
of Vapor-Deposited Ice on Au(111) at Low Temperatures," American Physical Society, Seattle, WA, Mar. 16, 2001.

B. D. Kay, Z. Dohnálek, G. A. Kimmel, K. P. Stevenson, and R. S. Smith, "Molecular Beam Studies of Nanoscale Films of Amorphous Solid Water," Chemical Engineering Seminar, University of Texas, Austin, TX, Oct. 24, 2000.* 
B. D. Kay, P. Ayotte, J. L. Daschbach, Z. Dohnálek, G. A. Kimmel, K. P. Stevenson, and R. S. Smith, G. R. Teeter, "Molecular Beam Studies of Nanoscale Films of Amorphous Solid Water," Gordon Conference n Chemical Reactions at Surfaces, Ventura, CA, Feb. 18-22, 2001.*

B. D. Kay, P. Ayotte, J. L. Daschbach, Z. Dohnálek, G. A. Kimmel, K. P. Stevenson, and R. S. Smith, G. R. Teeter, "Molecular Beam Studies of Nanoscale Films of Amorphous Solid Water," $221^{\text {st }}$ Annual Meeting, American Chemical Society, San Diego, CA, April 1-5, 2001.*

G. A. Kimmel, Z. Dohnálek, R. S. Smith, and B.D. Kay, "The Role of Two-Dimensional Compressibility in Physisorption, Competitive Adsorption, and Dynamic Displacement," 47th International Symposium, American Vacuum Society, Boston, MA, Oct. 1-5, 2000.*

A. Laskin, M. J. Iedema, S. A. Joyce, D. J. Gaspar, and J. P. Cowin, "Time-tagged Chemical Characterization of Atmospheric Aerosols," American Geophysical Union Fall Meeting, San Francisco, CA, Dec. 19, 2000.

P. H. Lommerse, G. S. Harms, H. Spaink, E. Snaar-Jagalska, G. Blab, and T. Schmidt, "Mobility of Lipid-Anchored Proteins in a Cell Membrane Visualized by Single-Molecule Spectroscopy," Annual Meeting, American Biophysical Society, Boston, MA, Feb. 18, 2001.

H. P. Lu, "Single-Molecule DNA Damage Recognition," Photophysics and Photochemistry 2000, Oeiras, Portugal, Oct. 19-21, 2000.

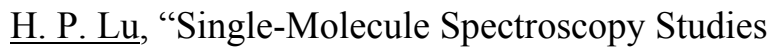
of the Mechanisms and Dynamics of NonCovalent and Covalent Molecular Interactions," Photochemistry and Photophysics 2000, October 2000.

H. P. Lu and Y. Chen, "Single-Molecule Spectroscopy Study of the Mechanisms and Dynamics of Non-Covalent and Covalent Molecular Interactions," Annual Meeting, Biophysical Society, Boston, MA, Feb. 20, 2001. Abstract: Biophys. J. 80, 340A (2000).

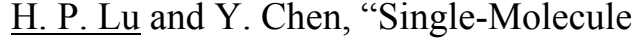
Spectroscopy Study of the Mechanisms and Dynamics of Molecular Interactions and Enzymatic Reactions," 221st Annual Meeting, American Chemical Society, San Diego, CA, Apr. 1-5, 2001.

H. P. Lu, "Single-Molecule Protein Dynamics," Nanoscale Science and Technology Workshop Seattle, WA, August 2001.*

G. Orr, M. Montal, B. D. Thrall, S. D. Colson, and H. P. Lu, "Single-Channel Patch-Clamp Recording Coupled with Linear and Nonlinear Confocal Scanning Fluorescence Spectroscopy: Towards the Simultaneous Probing of Single-Ion Channel Conformational Changes and Channel Kinetics," Annual Meeting, Biophysical Society, Boston, Feb. 18, 2001. Abstract: Biophys. J. 80, 151A (2000).

N. G. Petrik, G. A. Kimmel, and J. P. Cowin, "Radiolysis of Adsorbed Water Nanofilms," Gordon Research Conference, Andover, NH, Aug. 14, 2001.

M. S. Resat, G. A. Kimmel, J. H. Miller, J. C. McDonald, M. K. Murphy, D. J. Strom, N. F. Metting, B. D. Thrall, and S. D. Colson, "Spatially Resolved Single Cell Irradiator for Studying the Effects of Low-LET Radiation," Gordon Research Conference, Ventura, CA, 2001.

M. S. Resat, G. A. Kimmel, J. H. Miller, J. C. McDonald, M. K. Murphy, D. J. Strom, N. F. Metting, B. D. Thrall, and S. D. Colson, "Spatially Resolved Single Cell Irradiator for Studying the Effects of Low-LET Radiation," $13^{\text {th }}$ Symposium on Microdosimetry (MICROS), Stresa, Italy May 27 - June 1, 2001.

R. L. Sams, T. A. Blake, and S. W. Sharpe, "Cool Spectroscopy Using High Resolution Fourier Transform Infrared Spectroscopy," Optical Society of America Topical Meeting on Fourier Transform Spectroscopy and Optical Remote Sensing of the Atmosphere, Coeur d'Alene, ID, Feb. 5-8, 2001. 
S. W. Sharpe, R. L. Sams, T. J. Johnson, and P. M. Chu, "Intensity Artifacts in Gas-Phase FTIR Spectroscopy: Focus on the Aperture," Optical Society of America Topical Meeting on Fourier Transform Spectroscopy and Optical Remote Sensing of the Atmosphere, Coeur d'Alene, ID Feb. 5-8, 2001.

G. R. Teeter, Z. Dohnálek, S. Smith, G. A. Kimmel, and B. D. Kay, "Crystallization Kinetics of Amorphous Solid Water on Hydrophobic and Hydrophilic Substrates," Gordon Research Conference on Dynamics at Surfaces, Andover, NH, August 2001.

G. R. Teeter, Z. Dohnálek, S. Smith, G. A. Kimmel, and B. D. Kay, "Crystallization Kinetics of Amorphous Solid Water on Hydrophobic and Hydrophilic Substrates," National Meeting, American Vacuum Society, San Francisco, CA, Oct. 30 - Nov. 1, 2001.

L. S. Wang, "Probing Solution Phase Chemistry in the Gas Phase: Photodetachment of Multiply Charged Anions," Department of Chemistry, Louisiana State University, Baton Rouge, LA, Oct. 9, 2000.*

L. S. Wang, "Probing the Electronic Structure of Clusters Using Photoelectron Spectroscopy: from Manmade Clusters to Nature's Clusters," Tenth International Symposium on Small Particles and Inorganic Clusters, Atlanta, GA, Oct. 11$15,2000{ }^{*}$

L. S. Wang, "Probing the Electronic Structure of Novel Clusters Using Anion Photoelectron Spectroscopy," Department of Chemistry, University of Kentucky, Lexington, KY, Nov. 10, 2000.*

L. S. Wang, "Probing Metal-Metal and MetalLigand Interactions in Singly and Multiply Charged Transition Metal Complexes in the Gas Phase Using Photodetachment Spectroscopy," 2000 International Chemical Congress of Pacific Basin Societies (Pacifichem 2000), Honolulu, HI, Dec. 14-19, 2000.*
L. S. Wang, "Photoelectron Spectroscopy of Doubly-Charged Anions: Intramolecular Coulomb Repulsion and Solvent Stabilization," 2000 International Chemical Congress of Pacific Basin Societies (Pacifichem 2000), Honolulu, HI, Dec. 14-19, 2000.

L. S. Wang, "Probing Solution Phase Chemistry in the Gas Phase Using Electrospray and Photoelectron Spectroscopy," Department of Chemistry, Iowa State University, Ames, IA, Jan. 6, 2001.*

L. S. Wang, "Photodetachment of Gaseous Multiply Charged Anions," Department of Chemistry, Kansas State University, Manhattan, KS, Feb. 8, 2001.*

L. S. Wang, "Photodetachment of Multiply Charged Anions in the Gas Phase," $6{ }^{\text {th }}$ Winter Gordon Research Conference on Gaseous Ions: Structures, Energetics, and Reactions, Ventura, CA, Feb. 25 - Mar. 2, 2001.*

L. S. Wang, "Photodetachment Photoelectron Spectroscopy of Multiply Charged Anions," 221st Annual Meeting, American Chemical Society, San Diego, CA, Apr. 1-5, 2001.*

L. S. Wang, "Photodetachment Spectroscopy of Cluster Anions," Department of Physics, University of Missouri-Rolla, Rolla, MO, May 10, 2001.*

L. S. Wang, "Photodetachment Photoelectron Spectroscopy of Multiply Charged Anions," 2001 Northwest Regional Meeting of the American Chemical Society (NORM 2001), Seattle, WA, June 14-17, 2001.*

L. S. Wang, "Probing the Electronic Structure of Metal Clusters Using Anion Photoelectron Spectroscopy," Department of Chemistry, Universidad Nacional Autonoma de Mexico, July 18,2001 .* $^{*}$

L. S. Wang, "Probing the Solvation of Complex and Multiply Charged Anions in the Gas Phase Using Photodetachment Photoelectron Spectroscopy," $222^{\text {nd }}$ ACS National Meeting, Chicago, IL, August 26-30, 2001.* 
L. S. Wang, "Probing Condensed Phase Phenomena in the Gas Phase: From Solid to Solution," Department of Physics, Washington State University, Pullman, WA, Sept. 18, 2001.*

L. S. Wang, "From Tetracoordinate Planar Carbon Molecules to All-Metal Aromatic Molecules," Department of Chemistry, Washington State University. Pullman, WA, Oct. 1, 2001.*

L. Zhu and H. P. Lu, "Spectral Fluctuation Dynamics of Site-Specific Surface Enhanced Raman Scattering," 221st Annual Meeting, American Chemical Society, San Diego, CA, Apr. 1-5, 2001.

\section{Patents}

M. L. Balmer, R. G. Tonkyn, A. Y. Kim, D. Jimenez, T. M. Orlando, and S. E. Barlow, "Materials for NOx Reduction in Conjunction with a Non-Thermal Plasma," invention disclosure filed Sept. 1998.

\section{Honors and Recognition}

Steve Barlow and Russ Tonkyn are members of a team recognized by the DOE Office of Transportation Technologies with the 2001 CIDI Combustion and Emission Control Program Special Recognition Award. The citation reads "in recognition of outstanding achievement in research for the NonThermal Plasma CRADA." The award was presented at a contractor meeting, June 2001 in Knoxville.

Steve Barlow and Russ Tonkyn are members of a team presented with an R\&D 100 Award for "Catalyst Materials for Plasma-Catalysis Engine Exhaust Treatment." October 2001 in Chicago
Bruce Kay was elected a Fellow of the American Physical Society, November 2000. This recognition goes to members who have made outstanding contributions in physics and is limited to no more than one half of one percent of the membership. The citation reads, "For his innovative use of molecular beams and laser spectroscopy to elucidate chemical kinetics and dynamics of sorption, diffusion, phase transformation, and solvation at environmentally-relevant aqueous and oxide interfaces." The announcement was published in the March 2001 issue of APS News.

Peter $\mathbf{L u}$ is serving on the program committee for the International Meeting on Hole Burning, Single Molecule, and Related Spectroscopies: Science and Applications (HBSM2001), Taipei, Nov. 1823, 2001.

Peter Lu is Co-chair of the "Protein Conformation, Dynamics and Assemblies-I" platform session at the 45th American Biophysical Society Annual Meeting (2001).

Rod McDowell was appointed to a four-year term as a Titular Member of the International Union of Pure and Applied Chemistry's Commission I.5 (Physical Chemistry Division, Commission on Molecular Structure and Spectroscopy) starting January 1998. He is responsible for preparing for publication a IUPAC Recommendations document, Notations and Conventions in Molecular Spectroscopy: Part 4. Vibrational-Rotational Spectroscopy.

Russ Tonkyn: see Special Recognition Awards under Steve Barlow.

Sunney Xie's work on "Optical Probes for Imaging Single Molecules" was cited in the DOE's "Decades of Discovery," a list of the 100 most important discoveries supported by the Office of Science: http://www.sc.doe.gov/ feature_articles_2001/June/Decades/index.html. 


\section{Collaborations}

External Collaborations

Type of collaborator

(1) = non-visiting;

(2) = short-term $(<1$ month);

(3) $=$ long-term ( $\geq 1$ month).

S. E. Barlow

Oak Ridge National Laboratory (S. Nunn) (1)

Vehicle Exhaust Treatment

S. E. Barlow, R. G. Tonkyn, and C. H. F. Peden

Ford Motor Co. (J. Hoard) $(1,3)$

General Motors (S. Schmieg) $(1,3)$

Daimler/Chrysler (D. Brooks) $(1,3)$

Vehicle Exhaust Treatment

K. M. Beck

University of Central Florida (R. Peale, PASS

Affiliated Scientist) (3)

Optically Detected Magnetic Resonance (ODMR)

and Time-Resolved FTIR of Immobilized Heavy

Metals on Calcite Surfaces

K. M. Beck

University of Washington (J. Rehr) (1)

EXAFS Studies of Metal Ions in Caliche

K. M. Beck

National Institute of Materials and Chemical

Research, Japan (T. Sasaki) (3)

Preparation and Characterization of $\mathrm{Pt} / \mathrm{SiO}_{2}$ and $\mathrm{Pt} / \mathrm{MgO}$ Functional Nanocomposite Films by Laser Ablation

K. M. Beck

University of Washington (E. Stern) $(1,3)$

Phase Transitions

J. P. Cowin

University of Colorado (G. B. Ellison) (2)

Soft-Landing Ions

J. P. Cowin

Washington State University (M. Dresser)

Associated Western Universities (D. Doering) (2)

Ferroelectric Ice

J. P. Cowin

Arrowhead University (M. C. Gallagher) (1)

Thin-Film $\mathrm{Al}_{2} \mathrm{O}_{3}$
J. P. Cowin

Washington State University (B. Pate) (2)

Soft-Landing Ion Studies

J. L. Daschbach

Lewis \& Clark College, Portland OR (Barbara

Balko)

An Electrochemical Investigation into the Origin of the Enhanced Reactivity of Clays in Contact with Iron Meta.

W. P. Hess

Vanderbilt University (R. F. Haglund, Jr.) (1)

Studies of Laser/Solids Interactions

W. P. Hess

University of Colorado (G. B. Ellison)

Photochemistry of Thin Molecular Films

W. P. Hess

Washington State University (J. T. Dickinson) (1)

Laser Ablation Characterization of Solids

W. P. Hess

University College, London (A. Shluger and P. Sushko) (1)

Theory of Laser Desorption from Ionic Materials

W. P. Hess and K. M. Beck

University of Central Florida (R. Peale) (3)

Reaction of Metal-Oxide Materials

W. P. Hess and K. M. Beck

National Institute for Materials Center (NIMC), Tsukuba, Japan (T. Sasaki) $(1,3)$

Reaction of Metal-Oxide Materials

W. P. Hess and K. M. Beck

State University of New York at Stony Brook (R. Reeder) $(1,2)$

Metal Uptake by Carbonates in the Hanford

Vadose Zone

W. P. Hess

Reed College (Dan Gerrity)

Laser Ablation Studies on Oxides

W. P Hess

Sharp Laboratories of America, Inc.

(Yoshi Ono) (1)

Oxygen-Atom Oxidation of Silicon 
A. G. Joly

Nomadics (Wei Chen) and Univ. of California, Santa Cruz (Jin Zhang)

Up-Conversion Luminescence of $\mathrm{Mn}^{2+}$ in $\mathrm{ZnS}: \mathrm{Mn}^{2+}$ Nanoparticles

B. D. Kay

University of Washington (Hannes Jonsson) $(1,3)$

Growth, Structure, and Reactivity of Amorphous Ice

B. D. Kay

Chalmers University, Sweden

(Matts Persson) $(1,3)$

Fundamental Theoretical Studies of Gas Adsorption

B. D. Kay

University of Sherbrooke, Quebec, Canada (Patrick Ayotte)

Experimental Studies of Processes in Nanoscale Ice Films

B. D. Kay

University of Texas, Austin, Texas

(C. Buddie-Mullins)

Experimental Studies of Gas Phase Interactions with Ice Films

B. D. Kay

University of Washington, Seattle, WA

(Samuel C. Fain, Jr.)

Experimental Studies of the Morphology of ASW and Ice Surfaces

B. D. Kay

Air Force Research Laboratory at Hanscom Air Force Base (Al Viggiano)

Testing of Materials for the US Space Shuttle Program

R. S. McDowell

IUPAC Commission on Molecular Structure and Spectroscopy (1)

Notation and Conventions in Rotation-Vibration Spectroscopy

H. P. Lu

Scripps Research Institute (Klaus Hahn)

Protein-Protein Interaction Dynamics in CellSignaling
H. P. Lu

University of California San Diego

(Maurice Montal)

Single-Ion-Channel Dynamics

H. P. Lu

University of Oregon (Brian Matthews)

Single Molecule Enzymatic Reaction Dynamics

S. W. Sharpe

Oregon State University (J. W. Nibler) (2)

Jet Spectroscopy of Metal Borohydrides

S. W. Sharpe

State University of New York at Stony Brook (P. Varanasi) (1)

High-Resolution Infrared Spectroscopy of Atmospheric Interest

S. W. Sharpe

University of Colorado (V. Vaida) (2)

Spectroscopy of Water Clusters and Small

Droplets: Relevance to Atmospheric Radiative Transfer

S. W. Sharpe

Sandia National Laboratory (P. Hargis) (2)

Atmospheric Hydrolysis Chemistry

S. W. Sharpe

Wesleyan University (S. E. Novick) (1)

High-Resolution Spectroscopy of HydrogenBonded Dimers

S. W. Sharpe and T. A. Blake

National Institute for Standards and Technology

(G. T. Fraser and W. J. Lafferty) (1)

Rovibrational Spectral Analysis of Molecules and Molecular Clusters

S. W. Sharpe and T. A. Blake

Laboratoire de Photophysique Moléculaire du

CNRS, Paris (J.-M. Flaud) (1)

High-resolution Infrared Spectroscopy of Diborane

S. W. Sharpe and T. A. Blake

NASA Ames Research Center, Moffett Field, Cal. (C. Chackerian Jr.) (1)

Infrared Spectroscopy of Nitric Acid;

Near Infrared Laser Spectroscopy of Water Vapor 
L. S. Wang

Genentech, Inc., CA (J. B. Nicholas) (1),

Washington State University (X. Wang and

X. Yang), and EMSL TM\&S (E. R. Vorpagel)

Clusters and Multiply Charged Anions: Model

Studies and Solvations

L. S. Wang

Washington State University (X. B. Wang,

H. J. Zhai, and S. Liu)

University of Auckland, New Zealand

(P. Schwerdtfeger) (1)

Technical University of Berlin (D. Schroder and

H. Schwarz) (1),

Virginia Commonwealth University (G. L. Gutsev and P. Jena) (1)

Probing the Electronic Structure of Transition

Metal Clusters and Complexes

L. S. Wang

Washington State University (X. Li, H. F. Zhang, and H. J. Zhai)

Utah State University (A. I. Boldyrev) (1)

Iowa State University (J. D. Corbett) (1)

Investigation of Metal-Carbon Mixed Clusters and Aluminum Alloy Clusters

\section{Collaborations within PNNL}

S. E. Barlow

EHSD Materials and Chemical Sciences

Department, Nuclear Chemistry Section

(A. J. Peurrung)

Stabilization of Nonneutral Plasmas

S. E. Barlow

EHSD Atmospheric Sciences \& Global Change

Department, Atmospheric Sciences Section

(N. Laulainen)

Application of Aerosol Nucleation Techniques to

Ultrasensitive Contaminant Detection

J. P. Cowin

Atmospheric Sciences Department

(R. S. Disselkamp, C. M. Berkowitz)

Battelle Columbus (C. W. Spicer)

Atmospheric and Environmental Research, Inc.,

Boston (Y. Zhang)

Argonne National Laboratory (C. Bishop)

Effects of Heterogeneous Chemistry on $\mathrm{NO}_{y}$ in the

Troposphere
J. P. Cowin

Environment, Safety and Health Division,

Radiological Control Group (S. C. Marschman)

Dehydration and Radiolysis of Spent Nuclear

Fuels

W. P. Hess

EMSL Theory, Modeling, and Simulation

(S. S. Xantheas)

$\mathrm{HNO}_{3}\left(\mathrm{H}_{2} \mathrm{O}\right)_{\mathrm{n}}$ Spectroscopy and Calculations

G. R. Holtom

FSD Environmental Microbiology (Y. A. Gorby)

Analysis of Iron Reduction

G. R. Holtom

EMSL Macromolecular Structure \& Dynamics

(R. A. Wind)

Integrated Magnetic Resonance and Optical

Microscopy

G. R. Holtom

EMSL Macromolecular Structure \& Dynamics

(R. A. Wind)

Magnetic Resonance Microscopy of Mammalian Cells

A. G. Joly

Environmental Technology Division, Thermal

Processing Group (H. Li)

Laser-Induced Time-Resolved Fluorescence Spectroscopy of Lanthanide Element Chemical Environments in Alkali-Free Borosilicate Glasses

B. D. Kay

EMSL Interfacial and Processing Science (C. H. F. Peden)

Physics and Chemistry of Ceramic Surfaces

G. A. Kimmel and M. R. Sowa Resat

Fundamental Science Division, Molecular

Biosciences (B. D. Thrall and L. Kathman)

Environmental Technology Risk Analysis and

Health Protection (D. J. Strom)

A Novel, Spatially resolved cell Irradiator to Study

Bystander and Adaptive Responses to Low-LET

Radiation"

H. P. Lu

EHSD Molecular Biosciences Department

(E. J. Ackerman)

Single-Molecule DNA Damage Recognition 
H. P. Lu

EMSL Theory, Modeling, and Simulation

(G. K. Schenter)

Theory of Single-Enzyme Dynamics

S. W. Sharpe

National Security Division (R. G. Clemmer and

S. W. Martin)

Standoff Infrared Sensors for Detection of

Clandestine Methamphetamine Production

S. W. Sharpe

Energy Division, Engineering and Analytical

Sciences Department, Sensors and Measurement

Systems Section (J. S. Hartman) (1)

Development of Infrared Sniffer System

S. W. Sharpe

Energy Division, Engineering and Analytical

Sciences Department, Sensors and Measurement

Systems Section (J. W. Griffin)

Infrared Analysis in Head-Space Sampling for

Paper and Pulp Digesters

S. W. Sharpe

Energy Division, Engineering and Analytical

Sciences Department, Sensors and Measurement Systems Section (J. S. Hartman and

M. A. Khaleel)

Sensors and Modeling for Auto Glass Production
S. W. Sharpe

Energy Division, Engineering and Analytical

Sciences Department, Sensors and Measurement

Systems Group (J. F. Kelly) (2)

Spectroscopic Techniques for Atmospheric

Monitoring

L. S. Wang

Genentech, Inc., CA (J. B. Nicholas) (1)

Washington State University (X. Wang and $\mathrm{X}$. Yang)

EMSL Theory Modeling and Simulation (E. R. Vorpagel)

Clusters and Multiply Charged Anions: Model Studies and Solvations

L. S. Wang and S. D. Colson

EMSL Theory, Modeling, and Simulation (J. B. Nicholas)

Cluster Model Studies of EnvironmentallyImportant Oxide Clusters 


\section{Publications and Presentations from January 2002 to September 2002}

\section{Publications}

P. Ayotte, R. S. Smith, G. R. Teeter, Z. Dohnalek, G. A. Kimmel, and B. D. Kay, "A Beaker Without Walls: Formation of Deeply Supercooled Binary Liquid Solutions of Alcohols from Nanoscale Amorphous Solid Films," Phys. Rev. Lett. 88: $125-$ 128 (2002).

S. E. Barlow, A. E. Taylor, and K. Swanson, "Analytic Potentials for Realistic Electrodes," NonNeutral Plasma Physics IV, 606: 519-524 (2002).

T. A. Blake and P. L. Gassman, "Detection of Soil Surface Contaminants by Infrared Reflectance Spectroscopy," SPIE Proceedings, 4577: 239-261 (2002).

C. P. G. Butcher, B. F. G. Johnson, J. S. McIndoe, $\mathrm{X}$. Yang, X. B. Wang, and L. S. Wang, "CollisionInduced Dissociation and Photodetachment of Singly and Doubly Charged Anionic Polynuclear Transition Metal Carbonyl Clusters: $\mathrm{Ru} 3 \mathrm{Co}(\mathrm{CO})(13)(-), \mathrm{Ru} 6 \mathrm{C}(\mathrm{CO})(16)(2-)$, and Ru-6(CO)(18)(2-)," J. Chem. Phys. 116 (15): 65606566 2002).

W. Chen, F. Su, G. Li, A. G. Joly, J.-O. Malm, and J.-O. Bovin, "Temperature and Pressure Dependencies of the $\mathrm{Mn}^{2+}$ and Donor-Acceptor Emissions in ZnS: $\mathrm{Mn}^{2+}$ Nanoparticles," J. Appl. Phys., 92 (4): 1950-1955 (2002).

W. Chen, A. G. Joly, C. M. Kowalchuk, J.-O. Malm, Y. Huang, and J.-O. Bovin, "Structure, Luminescence, and Dynamics of Eu2O3 Nanoparticles in MCM-41," J. Phys. Chem. B, 106 (28): 7034-7041 (2002).

V. M. Devi, D. C. Benner, L. R. Brown, M.A.H. Smith, C. P. Rinsland, R. L. Sams, and S. W. Sharpe, "Multispectrum Analysis of Self- and $\mathrm{N}_{2}-$ Broadening, Shifting and Line Mixing Coefficients in the $v_{6}$ Band of ${ }^{12} \mathrm{CH}_{3} \mathrm{D}$," J. Quant. Spect. and Rad. Trans. 72 (2): 139-191 (2002).
Z. Dohnálek, G. A. Kimmel, D. E. McCready, J. S. Young, A. Dohnálková, R. S. Smith, and B. D. Kay, "Structural and Chemical Characterization of Aligned Crystalline Nanoporous MgO Films Grown Via Ballistic Deposition Conditions," J. Phys. Chem B, 106: 3526-3529 (2002). (Cover)

Z. Dohnálek, R. S. Smith, and B. D. Kay, “Adsorption Dynamics and Desorption Kinetics of Argon and Methane on $\mathrm{MgO}(100)$, , J. Phys. Chem. 106 (33): 8360-8366, 2002.

E. J. Elzinga, R. J. Reeder, S. H. Withers, R. E. Peale, R. A. Mason, K. M. Beck, and W. P Hess, "XAFS Study of Rare Earth Element Coordination in Calcite," Geochim. et Cosmochim. Acta, 66 (16): 2875-2885 (2002).

G. S. Harms, G. Orr, M. Montal, B. D. Thrall, S. D. Colson, and H. P. Lu, "Combined PatchClamp Recording and Single-Molecule Imaging Microscopy Study of Single-Molecule Ion Channel Dynamics," Biophysical Journal, 82 (1): 942 Part 2, (2002).

W. P. Hess, A. G. Joly, D. P. Gerrity, K. M. Beck, P.V. Sushko, and A. L. Shluger, "Control Of Laser Desorption Using Tunable Single Pulses and Pulse Pairs," J. Chem. Phys., 116 (18): 8144-8151 (2002).

W. P. Hess, A. G. Joly, K. M. Beck, D. P. Gerrity, Peter V. Sushko, and A. L. Shluger, "Solid-State Halogen Atom Source for Chemical Dynamics and Etching." Appl. Phys. Lett., 81 (6): 1140-1142, (2002).

W. P. Hess, A. G. Joly, K. M. Beck, and J. T. Dickinson "Time-Resolved Femtosecond Laser Desorption from Wide Band-Gap Single Crystals," Proc. SPIE Vol. 4760: 381 (2002).

D. H. Hu, Y. Chen, E. R. Vorpagel, and H. P. Lu, "Single-Molecule Conformational Mode-Specific Dynamics Under Enzymatic Reaction," Biophys. J., 82 (1): 139a (2002).

A. G. Joly, W. P. Hess, K. M. Beck, J. T. Dickinson, P. V. Sushko, and A. L. Shluger, "Femtosecond Time-Resolved Photostimulated Desorption From Ionic Crystals," Appl. Surf. Sci., 186: (1-4): 339344 (2002).

T. J. Johnson, R. L. Sams, T. A. Blake,

S. W. Sharpe, and P. M. Chu, "Removing Aperture- 
Induced Artifacts From Fourier Transform Infrared Intensity Values," Applied Optics, 41 (15): 28312839 (2002).

A. Laskin and J. P. Cowin, "On Deposition Efficiency of Point-to-Plate Electrostatic Precipitator," J. Aerosol Science, 33 (3): 405-409 (2002).

J. Laskin, E. V. Denisov, A. K. Shukla, S. E. Barlow, and J. H. Futrell, "Surface-Induced Dissociation in a Fourier Transform Ion Cyclotron Resonance Mass Spectrometer: Instrument Design and Evaluation," Anal. Chem., 74 (14): 3255-3261 (2002).

X. Li and L. S. Wang, "Experimental Search and Characterization of Icosahedral Clusters: A112X$(\mathrm{X}=\mathrm{C}, \mathrm{Ge}, \mathrm{Sn}, \mathrm{Pb}), "$ Phys. Rev. B, 65 (15): art. no. 153404 (2002).

X. Li, H. J. Zhai, and L. S. Wang, "Photoelectron Spectroscopy of Pentaatomic Tetracoordinateplanar Carbon Molecules: CAl3Si- and CAl3Ge-," Chem. Phys. Lett. 357 (5-6). 415-419 (2002).

X. F. Liao and L. S. Wang, "Comments on "Robust Stability for Interval Hopfield Neural Networks with Time Delay," IEEE Trans. on Neural Networks, 13 (1): 250-251 (2002).

S. R. Liu, H. J. Zhai, and L. S. Wang, "s-d Hybridization and Evolution of the Electronic and Magnetic Properties in Small Co and Ni Clusters," Phys. Rev. B 65 (11): 99-102, (2002).

T. B. McCord, G. R. Teeter, G. B. Hansen, M. T. Sieger, and T. M. Orlando, "Brines Exposed to Europa Surface," J. Geophysical ResearchPlanets 107 (E1): 61-66 (2002).

P. R. McCurdy, W. P. Hess, and S. Xantheas, "Nitric Acid-Water Complexes: Theoretical Calculations and Comparison to Experiment," J. Phys. Chem. A 106 (33): 7628-7635 (2002).

V. B. Mikheev, P. M. Irving, N. S. Laulainen, S. E. Barlow, and V. V. Pervukhin, "Laboratory Measurement of Water Nucleation Using a Laminar Flow Tube Reactor," J. Chem. Phys. 116 (24): 10772-10786 (2002).
G. Orr, G. S. Harms, B. D. Thrall, M. Montal, S. D. Colson, and Lu HP, "Probing Single-Molecule Ligand-Channel Interaction Dynamics in a Living Cell," Biophys. J. 82 (1): 255a (2002).

M. R. Papantonakis, J. Kim, W. P. Hess, and R. F. Haglund, Jr., "What Do Matrix-Assisted Laser Desorption/Ionization Mass Spectra Reveal About Ionization Mechanisams?" J. of Mass Spect., 37: 639-647 (2002).

R. L. Redington and R. L. Sams, "State-Specific Spectral Doublets in the FTIR Spectrum of Gaseous Tropolone," J. Phys. Chem. A, 106 (33): 7494-7511 (2002).

S. W. Sharpe, R. L. Sams, T. J. Johnson, P. M. Chu, G. C. Rhoderick, and F. R. Guenther, "Creation of $0.10 \mathrm{~cm}(-1)$ Resolution, Quantitative, Infrared Spectral Libraries for Gas," Vibrational Spectroscopy-Based Sensor Systems 4577: 12-24 (2002).

V. Shutthanandan, S. Thevuthasan, R. S. Disselkamp, A. Stroud, A. Cavanagh, E. M. Adams, D. R. Baer, L. A. Barrie, S. S. Cliff, and M. Jimenez-Cruz, "Development of PIXE, PESA and Transmission Ion Microscopy Capability to Measure Aerosols by Size and Time," Nuclear Instruments \& Methods in Physics Research Section B-Beam Interactions With Materials And Atoms, 189: 284-288 (2002).

R. S. Smith, Z. Dohnálek, G. A. Kimmel, K. P. Stevenson, and B. D. Kay, "The Relationship Between the Self-Diffusivity of Supercooled and Amorphous Solid Water," in Liquid Dynamics: Into the New Millennium, ACS Symposium Series, 820: ch. 15, 198-211 (2002).

R. S. Smith, Zdenek Dohnálek, G. A. Kimmel, K. P. Stevenson, and B. D. Kay, "The Continuity between ASW and Supercooled Liquid Water", ed. J. T. Fourkas, ACS Symposium Series 820: Liquid Dynamics, Experiment, Simulation and Theory, 198-211 (2002).

R. S. Smith, Z. Dohnalek, G. A. Kimmel, G. Teeter, P. Ayotte, J. Daschbach, and B. D. Kay, "Molecular Beam Studies of Nanoscale Films of Amorphous Solid Water" Water in Confining Geometries. Springer-Verlag, (2002). 
X. B. Wang, X. Yang, L. S. Wang, and J. B. Nicholas, "Photodetachment and Theoretical Study of Free and Water-Solvated Nitrate Anions, NO3-(H2O)(n) (n=0-6)," J. Chem. Phys., 116 (2): 561-570 (2002).

R. A. Wind, P. D. Majors, K. R. Minard, E. J. Ackerman, D. S. Daly, G. R. Holtom, B. D. Thrall, and T. J. Weber, "Combined Confocal and Magnetic Resonance Microscopy," Applied Magnetic Resonance 22 (2): 145-158, (2002).

S. Yoon, A. G. Panov, R. G. Tonkyn, A. C. Ebeling, S. E. Barlow, and M. L. Balmer, "An Examination of the Role of Plasma Treatment for Lean Nox Reduction Over Sodium Zeolite Y and Gamma Alumina Part I. Plasma Assisted NOx Reduction Over $\mathrm{NaY}$ and $\mathrm{Al}_{2} \mathrm{O}_{3}$," Catalysis Today, 72: 243 250 (2002).

S. Yoon, A. G. Panov, R. G. Tonkyn, A. C. Ebeling, S. E. Barlow, and M. L. Balmer, "An Examination of the Role of Plasma Treatment for Lean Nox Reduction Over Sodium Zeolite Y and Gamma Alumina Part 2. Formation of Nitrogen," Catalysis Today, 72: 243-250 (2002).

H. J. Zhai, L. S. Wang, A. E. Kuznetsov, and A. I. Boldyrev, "Probing the Electronic Structure and Aromaticity of Pentapnictogen Cluster Anions $\mathrm{Pn}(5)(-)(\mathrm{Pn}=\mathrm{P}, \mathrm{As}, \mathrm{Sb}$, and $\mathrm{Bi})$ Using Photoelectron Spectroscopy and $\mathrm{Ab}$ Initio Calculations, $J$. Phys. Chem. A 106 (23). 5600-5606 (2002).

H. J. Zhai, X. Yang, X. B. Wang, L. S. Wang, B. Elliott, and A. I. Boldyrev, "In Search Of Covalently Bound Tetra- And Penta-Oxygen Species: A Photoelectron Spectroscopic And Ab Initio Investigation Of MO4- and MO5- $(\mathrm{M}=\mathrm{Li}$, $\mathrm{Na}, \mathrm{K}, \mathrm{Cs})$," J. American Chemical Society, 124 (23): 6742-6750 (2002).

H. F. Zhang, A. C. Dohnalkova, C. M. Wang, J. S. Young, E. C. Buck, and L. S. Wang, "LithiumAssisted Self-Assembly Of Aluminum Carbide Nanowires And Nanoribbons," Nano Lett. Amer. Chem. Soc., 2: 105-108 (2002).

\section{In Press}

P. Ayotte, S. B. Nielsen, G. H. Weddle, M. A. Johnson, and S. S. Xantheas, "Spectroscopic Observation of Ion-Induced Water Dimer Desorp- tion in the $\mathrm{X}-\left(\mathrm{H}_{2} \mathrm{O}\right)_{2}(\mathrm{X}=\mathrm{F}, \mathrm{Cl}, \mathrm{Br}, \mathrm{I})$ Clusters," $J$. Phys. Chem. A, in press.

S. E. Barlow and M. D. Tinkle, "Linearizing an ICR cell," Rev. os Sci. Ins., in press.

K. M. Beck, A. G. Joly, W. P. Hess, D. P. Gerrity, N. F. Dupuis, A. L. Shluger, and P. V. Sushko, "Transient Center Photodecomposition in Potassium Bromide," App. Surf. Sci. in press.

Z. Dohnálek, G. A. Kimmel, P. Ayotte, R. S. Smith, and B. D. Kay, "The Deposition Angle Dependent Morphology of Amorphous Solid Water Films," $J$. Chem. Phys. in press.

L. P. Cramer, S. C. Lanford, W. P. Hess, and J. T. Dickinson. "Interactions Of Wide Bandgap Single Crystals With 157-Nm Excimer Laser Radiation I. $\mathrm{NaNO}_{3}$." Appl. Surf. Sci. in press.

M. Henyk, A. G. Joly, K. M. Beck, and W. P. Hess "Photon Stimulated Desorption From KI: Laser Control Of I-Atom Velocity Distributions" Surf. Sci. in press.

G. R. Holtom, B. Thrall, T. Weber, L. Zhu, D. Hopkins, C. Parkinson, S. Colson, J. Price, B. Y. Chin, M. E. Choi, and T. Risby, "Application of Coherent Anti-Stokes Raman Scattering (CARS) to Imaging Mammalian Cells: A Means for Gaining Molecular Selectivity in Multiphoton Imaging," Proc. SPIE, in press.

D. $\mathrm{Hu}$ and H. P. Lu, "Single-Molecule Nanosecond Anisotropy Dynamics of Tethered Protein Motions," J. Phys. Chem., in press.

A. Laskin, M. J. Iedema, and J. P. Cowin, "TimeTagged Archive of Field Collected Aerosols for Single Particle Analysis for the Texas 2000 Study," J. Aerosol Sci., submitted.(LDRD)

P. D. Majors, K. R. Minard, E. J. Ackerman, G. R. Holtom, D. F. Hopkins, C. I. Parkinson, T. J. Weber, and R. A. Wind, "A Combined Confocal And Magnetic Resonance Microscope For Biological Studies," Rev. Sci. Instr., in press.

M. R. Papantonakis, J. Kim, W. P. Hess, and R. F. Haglund, Jr., "What Do MALDI Mass Spectra Reveal About Ionization Mechanisms?" J. Mass Spectrom. in press. 
Y. D. Suh, G. K. Schenter, L. Zhu, and H. P. Lu, "Probing Nano-Surface Enhanced Raman Scattering Fluctuation Dynamics using Correlated AFM and Confocal Ultramicroscopy," Ultramicroscopy, in press.

R. G. Tonkyn, S. E. Barlow and J. W. Hoard, "Reduction of NOx in Synthetic Diesel Exhaust via Two-Step Plasma-Catalysis Treatment," Applied Catalysis B: Environmental, in press

R. G. Tonkyn, S. E. Barlow, S. Yoon, A. Panov, A. Ebeling, and M. L. Balmer, "Lean NOx Reduction by Plasma Assisted Catalysis," Proc. DEER 2000 , in press.

L. S. Wang, "Photodetachment of Multiply-Charged Anions," Comments Atomic Mol. Phys., in press.

L. S. Wang, "Clusters and Nanoscale Structures," Encyclopedia of Chemical Physics and Physical Chemistry, ed. by J. H. Moore and N. D. Spencer IOP Publishing Inc., Philadelphia, 2000, in press.

S. H. Withers, G. Braunstein, R. E. Peale, K. M. Beck, W. P Hess, and R. J. Reeder, "Broad Distribution of Crystal Field Environments for $\mathrm{Nd}^{3+}$ in Calcite." Geochim. Cosmochim. Acta in press.

R. G. Tonkyn, S. E. Barlow and J. W. Hoard, "Reduction of NOx in Synthetic Diesel Exhaust via Two-Step Plasma-Catalysis," Appl. Cat. B:

Environ., 1240 2002, in press

\section{Presentations}

Presenter Underlined $\quad *=$ Invited Talk

Z. Dohnálek, "Synthesis and Characterization of Nanoporous Thin Films Using Molecular Beams," Seminar at Seagate Research Center, Pittsburgh, PA, January 2002.*

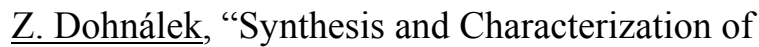
Nanoporous Thin Films Using Molecular Beams," Seminar at Surface Science Center, Department of Chemistry, University of Pittsburgh, Pittsburgh, PA, January 2002.*
Z Dohnálek, G. A. Kimmel, P. Ayotte, J. Dashbach, K. P. Stevenson, G. Teeter, R. S. Smith, and B. D. Kay, "Molecular Beam Studies of Nanoscale Films of Amorphous Solid Water," 224 $4^{\text {th }}$ National Meeting, American Chemical Society, Boston MA, August 18-22, 2002.

G. S. Harms, G. Orr, M. Montal, B. D. Thrall, S. D Colson, and H. P. Lu, "Combined Patch-Clamp Recording and Single-Molecule Imaging Microscopy Study of Single-Molecule Ion Channel Dynamics," Biophysical Society $46^{\text {th }}$ Annual Meeting, San Francisco, CA, February 23, 2002.*

G. S. Harms, G. Orr, B. D. Thrall, S. D. Colson, M. Montal, and H. P. Lu, "Simultaneous PatchClamp Recording and Single-Molecule Imaging Study of Single-Molecule Ion Channel Dynamics," American Physics Society Meeting, Indianapolis, IA March 18, 2002.*

W. P. Hess, "Femtosecond Time-Resolved Laser Desorption From Wide Band-Gap Single Crystals." High Power Laser Ablation 2002, Taos, NM, ril 2002.

W. P. Hess, A. G. Joly, K. M. Beck, J. T. Dickinson, P. V. Sushko, and A. L. Shluger, "Photostimulated Desorption From Crystalline Solids: Laser Control Of Product States Using Femtosecond And Nanosecond Lasers," University College, London, June, 2002.

W. P. Hess, A. G. Joly, K. M. Beck, D. P. Gerrity, P. V. Sushko, and A. L. Shluger, "Laser Control Of Photostimulated Desorption Using Selcted Photon Energies And Sequential Pulse Pairs," The $9^{\text {th }}$ Workshop On Desorption Induced By Electronic Transitions (DIET9), Aussios, France, June, 2002.

B. D. Kay, P. Ayotte, J. Daschbach, Z. Dohnálek, G. A. Kimmel, K. P. Stevenson, R. S. Smith and G. Teeter "Molecular Beam Studies of Nanoscale Films of Amorphous Solid Water," Physical Chemistry Seminar, University of Liverpool, Liverpool, England, January 7, 2002.*

B. D. Kay, P. Ayotte, J. Daschbach, Z. Dohnálek, G. A. Kimmel, K. P. Stevenson, R. S. Smith and G. Teeter, Dynamics and Kinetics of Water Vapor Condensation on Ice and Liquid Water," Instructor at Young Researcher Workshop, Center of Physics, Les Houches, France, January 8-13, 2002.* 
B. D. Kay, P. Ayotte, J. L. Daschbach, Z. Dohnálek, G. A. Kimmel, K. P. Stevenson, R. S. Smith and G. Teeter, "Molecular Beam Studies of Nanoscale Films of Amorphous Solid Water," Research Conference on Surface Photoprocesses, Center of Physics, Les Houches, France, January 14-18, 2002.*

B. D. Kay, Z. Dohnálek, R. S. Smith, G. Kimmel and S. A. Joyce, "Molecular Beam Synthesis and Characterization of Defects on Nanoscale Films of MgO," Joint Environmental Catalysis and Physical Chemistry Seminar, Northwestern University, Evanston, IL March 8, 2002.*

B. D. Kay, P. Ayotte, J. L. Daschbach, Z. Dohnálek, G. A. Kimmel, K. P. Stevenson, R. S. Smith and G. Teeter, "Molecular Beam Studies of Nanoscale Films of Amorphous Solid Water," European Physical Society Meeting, Brighton, England, April 7-11, 2002.*

B.D. Kay, Z. Dohnálek, R. S. Smith, G. A. Kimmel, and S. A. Joyce, "Molecular Beam Synthesis and Characterization of Defects on Nanoscale Films of $\mathrm{MgO}$," Surface Science Research Center, University of Liverpool, Liverpool, England, April 15, 2002.*

H. P. Lu, D. Hu, Y. Chen, E. R. Vorpagel, "SingleMolecule Probing the Energy Landscape of Enzymatic Reaction and Non-Covalent Interactions," American Physics Society Meeting, Indianapolis, IA, March 18, 2002.

$\underline{\mathrm{H}}$. Peter Lu, "Conformational dynamics of an ion channel by simultaneous single-molecule fluorescence and patch recording," The Gordon Research Conference of Ion Channels, Hilton, NH, July, 2002.*

P. D. Majors, T. J. Weber, G. R. Holtom, K. R. Minard, and R. A. Wind, "Combined Optical and Magnetic Resonance Microscopy of Heterogeneous JB6 Tumor Spheroid Populations,” 2002 Pittsburgh Conference, New Orleans, LA, March 18, 2002.*

G. Orr, G. S. Harms, B. D. Thrall, M. Montal, S. D. Colson, and H. P. Lu. "Probing SingleMolecule Ligand-Channel Interaction Dynamics in a Living Cell," Biophysical Society $46^{\text {th }}$ Annual Meeting, San Francisco, CA, February 23, 2002.*
R. G. Tonkyn and S. E. Barlow, Multi-Step Discharge/Catalyst Processing of NOx in Synthetc Diesel Exhaust, Spring 2002 SAE Fuels and Lubes Meeting, Reno, NV, May 6-9, 2002.

E. R. Vorpagel, H. P. Lu, D. Hu, Y. Chen, "Molecular Dynamics Simulations Help Understand Single-Molecule Spectroscopic Enzyme Hydrolysis," American Physics Society Meeting, Indianapolis, IA, March 18, 2002.

L. S. Wang, "Photodetachment of bare and solvated multiply charged anions," Gordon Research Conference on Molecular and Ionic Clusters, Ventura, CA, January 6-11, 2002.*

Z. Wang, C. C. Ainsworth, K. Wagnon, and A. G. Joly, "A fluorescence study of uranyl sorption and speciation at clay mineral surfaces," $223^{\text {rd }}$ ACS National Meeting, Orlando, FL, April 7-11, 2002.

J. N. Woodford, A. G. Joly, and D. A. Olsen, "Investigation of flavonoid and anthocyanidin molecules for potential optoelectronic applications," 223rd ACS National Meeting, Orlando, FL, April 7$11,2002$.

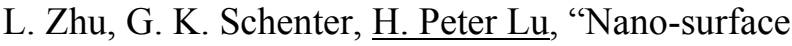
enhanced raman scattering fluctuation dynamics," The Scanning Probe Microscopy 2002, Las Vegas, NV, May 2002.

L. Zhu, G. K. Schenter, Y. D. Suh, M. Micic, N. A. Klymyshyn, H. Peter Lu, "Near-Field Enhanced Nano-Surface Enhanced Raman Scattering And Scanning Microscopy," Pacific Northwest Chapter of the American Vacuum Society $13^{\text {th }}$ Annual Symposium, Vancouver, WA, Sept. 2002.

A Laskin, C. M. Berkowitz, J. P. Cowin, D. J. Gaspar, M. J. Iedema, and C. W. Spicer, "Comprehensive field observations of sea salt chemistry over Houston/Galveston area", Conference on Atmospheric Chemistry: Urban, Regional, and Global Scale Impacts of Air Pollutants, Orlando, FL, January 13, 2002.*

W. Chen, A. G. Joly, C. M. Kowlachuk, J. Malm, Y. Huang, and J. Bovin, "Structure, luminescence, and dynamics of Eu2O3 nanoparticles in MCM-41", SPIE $47^{\text {th }}$ Annual Meeting, International Symposium on Optical Science and Technology, Seattle, WA, July 9,2002 * $^{*}$ 
W. Chen, A. G. Joly, J. Roark, “ Photostimulated Luminescence and Dynamics of $\mathrm{AgI}$ and $\mathrm{Ag}$ Nanoclusters in Zeolites", SPIE $47^{\text {th }}$ Annual Meeting, International Symposium on Optical Science and Technology, Seattle, WA, July 9, 2002.*

M. Henyk, W. P. Hess, A. G. Joly, K. M. Beck, P. V. Sushko, and A. L. Shluger, "Laser desorption of hyperthermal iodine atoms from $\mathrm{KI}$ in the absorption threshold region," Gordon Research Conference - Laser Interactions with Materials, Andover, NH, July 23, 2002.*

D. Hu, Y. Chen, E. R. Vorpagel, and H. Lu, "Singlemolecule conformational mode-specific dynamcs under enzymatic reaction," Biophysical Society $46^{\text {th }}$ Annual Meeting, San Francisco, CA, February 23, 2002.*
B. D. Kay, "Molecular beam synthesis and characterization of surface defects on nanoscale films of MgO," The Surface Science Research Centre, Liverpool, England, April 15, 2002.*

S. W. Sharpe, R. L. Sams, T. J. Johnson, P. M. Chu, G. C. Rhoderick, and F. R. Guenther, "Quantitativ infrared spectral libraries for gas samples," Collaboration with Dugway, Dugway, UT, May 9, 2002.*

S. W Sharpe, R. L. Sams, T. J. Johnson, P. M. Chu, G. C. Rhoderick, and F. R. Guenther, "Quantitative infrared spectral libraries for gas samples," Hitran 2002, Boston, MA, June 12, 2002.* 


\section{Acronyms and Abbreviations}

ACS American Chemical Society

ARM Atmospheric Radiation Measurement

Program

ARO U.S. Army Research Office

AWU Associated Western Universities

BES DOE Office of Basic Energy Sciences

CNR Italian National Research Council (Consiglio Nazionale della Ricerche)

CNRS Centre National de la Recherche Scientifique (France)

COE Cooperative Office Education

CRADA Cooperative Research and Development Agreement

CS\&D Chemical Structure and Dynamics

DARPA Defense Advanced Research Projects Agency

DOE U. S. Department of Energy

EHSD Environmental and Health Sciences Division

EMSL William R. Wiley Environmental Molecular Sciences Laboratory

EMSP DOE Environmental Management Science Program
FTIR Fourier-transform infrared

IUPAC International Union of Pure and Applied Chemistry

LDRD Laboratory Directed Research and Development

NASA National Aeronautics and Space Administration

NATO North Atlantic Treaty Organization

NSF National Science Foundation

ODMR optically detected magnetic resonance

PNNL Pacific Northwest National Laboratory

SABIT Special American Business Internship Training Program

USCAR U.S. Council for Automotive Research

VNIPIET Russian Research Institute of Complex Power Technology 
